\title{
Representação de funcionais lineares em hiper-ideais de operadores multilineares e de polinômios homogêneos
}

\author{
Raquel Maria Nogueira Wood Noronha
}

TESE APRESENTADA

$\mathrm{AO}$

Instituto DE MATEMÁtica E EstatísticA

DA

Universidade DE SÃo PAUlo

PARA

OBTENÇÃO DO TÍTULO

$\mathrm{DE}$

DOUTOR EM Cî̂NCIAS

Programa: Matemática

Orientador: Prof. Dr. Geraldo Márcio de Azevedo Botelho

Durante o desenvolvimento deste trabalho a autora recebeu auxílio financeiro da CAPES

São Paulo, Dezembro de 2021 


\section{Representação de funcionais lineares em hiper-ideais de operadores multilineares e de polinômios homogêneos}

Esta versão da tese contém as correções e alterações sugeridas pela Comissão Julgadora durante a defesa da versão original do trabalho, realizada em 21/12/2021. Uma cópia da versão original está disponível no Instituto de Matemática e Estatística da Universidade de São Paulo.

Comissão Julgadora:

- Prof. Dr. Geraldo Márcio de Azevedo Botelho (orientador) - FAMATUFU

- Prof. Dr. Joedson dos Santos - DM-UFPB

- Prof ${ }^{\mathrm{a}}$. Dr ${ }^{\mathrm{a}}$. Ximena Mujica-DMAT-UFPR

- Profa . Dr. Thiago Rodrigo Alves - ICE-UFAM

- Prof $^{\mathrm{a}}$. Dr ${ }^{\mathrm{a}}$. Mary Lilian Lourenço - IME-USP 


\section{Resumo}

Nesta tese introduzimos a hiper-transformada de Borel como ferramenta para representar funcionais lineares em hiper-ideais de operadores multilineares entre espaços de Banach. E também a hiper-transformada de Borel polinomial para representar funcionais lineares em hiper-ideais de polinômios homogêneos. Essas transformadas são aplicadas para representar funcionais lineares em espaços de operadores multilineares e polinômios homogêneos que são: aproximáveis por operadores/polinômios de posto finito, compactos, hiper- $(s ; r)$-nucleares e hiper- $\sigma(p)$-nucleares. Uma nova técnica para gerar hiper-ideais de operadores multilineares e hiper-ideais e ideais bilaterais de polinômios também é desenvolvida.

Palavras-chave: Espaços de Banach, hiper-ideais, representação de funcionais lineares, operadores multilineares, hiper-transformada de Borel, polinômios homogêneos, hipertransformada de Borel polinomial. 


\section{Abstract}

In this work we introduce the hyper-Borel transform as a tool to represent linear functionals on hyper-ideals of multilinear operators between Banach spaces. And also the polynomial hyper-Borel transform to represent linear functionals on hyper-ideals of homogeneous polynomials. These transforms are applied to represent linear functionals on spaces of multilinear operators and homogeneous polynomials which are: approximable by finite rank operators/polynomials, compact, hyper- $(s ; r)$-nuclear and hyper- $\sigma(p)$-nuclear. A new technique to generate hyper-ideals of multilinear operators and hyper-ideals and two-sided ideals of homogeneous polynomials is also developed.

Keywords: Banach spaces, hyper-ideals, representation of linear functionals, multilinear operators, hyper-Borel transform, homogeneous polynomials, polynomial hyperBorel transform. 


\section{Lista de Símbolos}

$\begin{array}{ll}\mathbb{N} & \text { conjunto dos números naturais } \\ \mathbb{R} & \text { conjunto dos números reais } \\ \mathbb{C} & \text { conjunto dos números complexos } \\ \mathbb{K} & \text { corpo dos escalares } \mathbb{R} \text { ou } \mathbb{C} \\ E, F, G, H & \text { espaços de Banach } \\ B_{E} & \text { bola fechada unitária }\{x \in E:\|x\| \leq 1\} \\ E^{*} & \text { dual topológico de } E \\ E^{\prime} & \text { dual algébrico de } E \\ J_{E} & \text { mergulho canônico } J_{E}: E \longrightarrow E^{* *} \\ \ell_{p}(E) & \text { espaço das sequências absolutamente } p \text {-somáveis a valo- } \\ \ell_{p}^{w}(E) & \text { res em } E \\ \ell_{\infty}(E) & \text { espaço das sequências fracamente } p \text {-somáveis a valores } \\ c_{0} & \text { espaço das sequências limitadas em } E \\ c_{0}^{w}(E) & \text { espaço das sequências de escalares que convergem para } \\ & \text { subespaço de } c_{0}(E) \text { formado pelas sequências fraca- } \\ c_{00} & \text { mente nulas. } \\ R a d(E) & \text { espaço das sequências de escalares eventualmente nulas } \\ \ell_{p}^{u}(E) & \text { conjunto de sequências quase incondicionalmente } \\ \ell_{p}\langle E\rangle & \text { somáveis em } E \\ & \text { subespaço de } \ell_{p}^{w}(E) \text { formado pelas sequências incondici- } \\ & \text { espaço das sequências Cohen fortemente } p \text {-somáveis em } \\ & E\end{array}$




\begin{tabular}{|c|c|}
\hline $\mathcal{L}(E ; F)$ & $\begin{array}{l}\text { espaço dos operadores lineares contínuos } \\
\text { de } E \text { em } F\end{array}$ \\
\hline$L\left(E_{1}, \ldots, E_{n} ; F\right)$ & $\begin{array}{l}\text { espaço vetorial dos operadores } \\
\text { multilineares de } E_{1} \times \cdots \times E_{n} \text { em } F\end{array}$ \\
\hline $\mathcal{L}\left(E_{1}, \ldots, E_{n} ; F\right)$ & $\begin{array}{l}\text { espaço vetorial dos operadores } \\
\text { multilineares contínuos de } E_{1} \times \cdots \times E_{n} \text { em } F\end{array}$ \\
\hline$L\left({ }^{n} E ; F\right)$ & $L(E, .(n) ., E ; F)$ \\
\hline $\mathcal{L}\left({ }^{n} E ; F\right)$ & $\mathcal{L}(E, \stackrel{(n)}{.}, E ; F)$ \\
\hline $\mathcal{L}\left(E_{1}, \ldots, E_{n}\right)$ & $\mathcal{L}\left(E_{1}, \ldots, E_{n} ; \mathbb{K}\right)$ \\
\hline$L^{s}\left({ }^{n} E ; F\right)$ & $\begin{array}{l}\text { subespaço vetorial de } L\left({ }^{n} E ; F\right) \text { das aplicações } \\
\text { multilineares simétricas }\end{array}$ \\
\hline $\mathcal{L}^{s}\left({ }^{n} E ; F\right)$ & $\begin{array}{l}\text { subespaço vetorial de } L\left({ }^{n} E ; F\right) \text { das aplicações } \\
\text { multilineares contínuas simétricas }\end{array}$ \\
\hline$x_{1} \otimes \cdots \otimes x_{n}$ & $\begin{array}{l}\text { tensor elementar: } x_{1} \otimes \cdots \otimes x_{n}(B)=B\left(x_{1}, \ldots, x_{n}\right) \text {, } \\
B \in \mathcal{L}\left(E_{1}, \ldots, E_{n}\right)\end{array}$ \\
\hline$\pi$ & norma projetiva em $E_{1} \otimes \cdots \otimes E_{n}$ \\
\hline$\pi_{s}$ & norma $s$-tensorial projetiva em $\hat{\otimes}_{\pi}^{n, s} E$ \\
\hline$E_{1} \hat{\otimes}_{\pi} \cdots \hat{\otimes}_{\pi} E_{n}$ & produto tensorial projetivo de $E_{1}, \ldots, E_{n}$ \\
\hline$\hat{\otimes}_{\pi}^{n, s} E$ & produto tensorial simétrico projetivo \\
\hline$\hat{\otimes}_{\pi_{s}}^{n, s} E$ & produto tensorial simétrico $s$-projetivo \\
\hline$E \hat{\otimes}_{\varepsilon} F$ & produto tensorial injetivo de $E$ e $F$ \\
\hline$A_{L}$ & linearização do operador multilinear $A$ \\
\hline$A \otimes y$ & $\begin{array}{l}\text { operador } n \text {-linear dado por } A \otimes y\left(x_{1}, \ldots, x_{n}\right)= \\
A\left(x_{1}, \ldots, x_{n}\right) y, A \in \mathcal{L}\left(E_{1}, \ldots, E_{n}\right) \text { e } y \in F\end{array}$ \\
\hline$x^{*} \otimes y$ & $\begin{array}{l}\text { operador linear dado por } x^{*} \otimes y(x)=x^{*}(x) y, x^{*} \in E^{*} \mathrm{e} \\
y \in F\end{array}$ \\
\hline $\mathcal{L}_{f}$ & classe dos operadores multilineares de tipo finito \\
\hline $\mathcal{L}_{\mathcal{F}}$ & classe dos operadores multilineares de posto finito \\
\hline $\mathcal{I}$ & ideal de operadores lineares \\
\hline$I d_{\mathbb{K}}$ & operador identidade em $\mathbb{K}$ \\
\hline $\mathcal{N}$ & ideal dos operadores lineares nucleares \\
\hline $\mathcal{J}$ & ideal dos operadores lineares integrais \\
\hline $\mathcal{M}$ & multi-ideal de aplicações multilineares \\
\hline $\mathcal{F}$ & ideal dos operadores lineares de posto finito \\
\hline$\overline{\mathcal{F}}$ & fecho de $\mathcal{F}$ \\
\hline
\end{tabular}




\begin{tabular}{|c|c|}
\hline $\mathcal{K}$ & ideal dos operadores lineares compactos \\
\hline $\mathcal{W}$ & ideal dos operadores lineares fracamente compactos \\
\hline $\mathcal{N}_{(s ; r)}$ & ideal dos operadores lineares $(s ; r)$-nucleares \\
\hline $\mathcal{N}_{\sigma(p)}$ & ideal dos operadores lineares $\sigma(p)$-nucleares \\
\hline $\mathcal{L}_{q \tau(p)}$ & classe dos operadores lineares quase- $\tau(p)$-somantes \\
\hline $\mathcal{L}_{\mathcal{N}}$ & classe dos operadores multilineares nucleares \\
\hline $\mathcal{L}_{\sigma(p)}$ & classe dos operadores multilineares $\sigma(p)$-nucleares \\
\hline $\mathcal{L}_{\mathcal{J}}$ & classe dos operadores multilineares integrais \\
\hline $\mathcal{I} \circ \mathcal{L}$ & $\begin{array}{l}\text { classe dos operadores multilineares obtida através dos } \\
\text { ideais de composição a partir do ideal } \mathcal{I}\end{array}$ \\
\hline $\mathcal{L}_{a s}^{\left(s ; r_{1}, \ldots, r_{n}\right)}$ & $\begin{array}{l}\text { classe dos operadores multilineares absolutamente } \\
\left(s ; r_{1}, \ldots, r_{n}\right) \text {-somantes }\end{array}$ \\
\hline $\mathcal{L}_{\mathcal{N}}^{\left(s ; r_{1}, \ldots, r_{n}\right)}$ & $\begin{array}{l}\text { classe dos operadores multilineares } \\
\left(s ; r_{1}, \ldots, r_{n}\right) \text {-nucleares }\end{array}$ \\
\hline $\mathcal{L}_{q \tau(p ; q)}$ & $\begin{array}{l}\text { classe dos operadores multilineares } \\
\text { quase- } \tau(p ; q) \text {-somantes }\end{array}$ \\
\hline $\mathcal{L}_{\mathcal{H} \mathcal{N}_{(s ; r)}}$ & $\begin{array}{l}\text { classe dos operadores multilineares } \\
\text { hiper- }(s ; r) \text {-nucleares }\end{array}$ \\
\hline $\mathcal{L}_{\mathcal{H N}}$ & classe dos operadores multilineares hiper-nucleares \\
\hline $\mathcal{L}_{\mathcal{H} \sigma(p)}$ & $\begin{array}{l}\text { classe dos operadores multilineares } \\
\text { hiper- } \sigma(p) \text {-nucleares }\end{array}$ \\
\hline $\mathcal{L}_{\mathcal{K}}$ & classe dos operadores multilineares compactos \\
\hline $\mathcal{L}_{\mathcal{W}}$ & $\begin{array}{l}\text { classe dos operadores multilineares fracamente compac- } \\
\text { tos }\end{array}$ \\
\hline $\mathcal{L}_{X_{1}, \ldots, X_{n} ; Y}$ & $\begin{array}{l}\text { classe dos operadores multilineares }\left(X_{1}, \ldots, X_{n} ; Y\right) \text { - } \\
\text { somantes }\end{array}$ \\
\hline $\mathcal{L}_{X ; Y}$ & classe dos operadores multilineares $(X ; Y)$-somantes \\
\hline $\mathcal{G}$ & $\begin{array}{l}\text { subclasse da classe dos operadores multilineares } \\
\text { contínuos entre espaços de Banach }\end{array}$ \\
\hline $\mathcal{H}$ & hiper-ideal dos operadores multilineares \\
\hline$P\left({ }^{n} E ; F\right)$ & $\begin{array}{l}\text { espaço vetorial sobre } \mathbb{K} \text { dos polinômios } \\
n \text {-homogêneos de } E \text { em } F\end{array}$ \\
\hline $\mathcal{P}\left({ }^{n} E ; F\right)$ & $\begin{array}{l}\text { espaço vetorial sobre } \mathbb{K} \text { dos polinômios } \\
n \text {-homogêneos contínuos de } E \text { em } F\end{array}$ \\
\hline$\hat{A}$ & $\begin{array}{l}\text { polinômio homogêneo associado à aplicação } \\
\text { multilinear } A\end{array}$ \\
\hline$\check{P}$ & $\begin{array}{l}\text { aplicação multilinear simétrica associada } \\
\text { ao polinômio homogêneo } P\end{array}$ \\
\hline
\end{tabular}


$P_{L}$

$P_{L, s}$

$\sigma_{n}: E \longrightarrow \hat{\otimes}_{\pi}^{n, s} E$

$\mathcal{P}_{f}$

$\mathcal{P}_{\mathcal{F}}$

$\varphi^{n} \otimes y$

$Q \otimes y$

$\mathcal{Q}$

$\mathcal{P}_{\mathcal{H N}}(s ; r)$

$\mathcal{P}_{\mathcal{H N}}$

$\mathcal{P}_{\mathcal{H} \sigma(p)}$

$\mathcal{I} \circ \mathcal{P}$

$\mathcal{P}_{X ; Y}$

$\beta_{n}$

$\beta$

$\mathcal{B}_{n}$

$\mathfrak{B}_{\mathfrak{n}}$

$\alpha$

$\mathcal{L}_{\alpha}\left(E ; F^{*}\right)$

$\operatorname{ker}\left(\mathcal{B}_{n}\right)$

$\operatorname{ker}\left(\mathfrak{B}_{n}\right)$ linearização do polinômio homogêneo $P$ com respeito à norma projetiva $\pi$

linearização do polinômio homogêneo $P$ com respeito à norma $s$-projetiva $\pi_{s}$

polinômio $n$-homogêneo canônico

classe dos polinômios homogêneos de tipo finito

classe dos polinômios homogêneos de posto finito

polinômio $n$-homogêneo dado por $\varphi^{n} \otimes y(x)=\varphi(x)^{n} y$

polinômio $n$-homogêneo dado por $Q \otimes y(x)=Q(x) y$

hiper-ideal de polinômios homogêneos

classe dos polinômios $n$-homogêneos hiper- $(s ; r)$ nucleares

classe dos polinômios $n$-homogêneos hiper-nucleares

classe dos polinômios $n$-homogêneos hiper- $\sigma(p)$ nucleares

classe de polinômios homogêneos obtida através dos ideais de composição a partir do ideal $\mathcal{I}$

classe dos polinômios homogêneos $(X ; Y)$-somantes

transformada de Borel

transformada de Borel linear

hiper-transformada de Borel

hiper-transformada de Borel polinomial

semi-ideal à esquerda

subespaço vetorial de $\mathcal{L}\left(E ; F^{*}\right)$ associado ao semi-ideal à esquerda $\alpha$

núcleo do operador linear $\mathcal{B}_{n}$

núcleo do operador linear $\mathfrak{B}_{n}$ 


\section{Sumário}

Resumo

Abstract $\quad$ iv

Lista de Símbolos $\quad$ v

Introdução

1 Preliminares $r$

1.1 Operadores lineares contínuos . . . . . . . . . . . . . . . . 9

1.2 Operadores multilineares contínuos . . . . . . . . . . . . . . 12

1.3 Ideais de operadores lineares . . . . . . . . . . . . . . . 17

1.4 Multi-ideais de operadores multilineares f . . . . . . . . . . . . . . . . . 19

1.5 Hiper-ideais de operadores multilineares . . . . . . . . . . . . 23

1.6 Polinômios homogêneos . . . . . . . . . . . . . . . . . . 25

2 Operadores Multilineares Hiper-Nucleares $\quad 31$

2.1 Operadores multilineares hiper- $(s ; r)$-nucleares . . . . . . . . . . . . . 31

2.2 Operadores multilineares hiper- $\sigma(p)$-nucleares . . . . . . . . . . . 36

3 Representação de Duais $\quad 50$

3.1 A hiper-transformada de Borel . . . . . . . . . . . . . . . . 52

3.2 Multi-ideais de composição . . . . . . . . . . . . . . . . . . . . . 61 
3.3 Aplicações . . . . . . . . . . . . . . . . . . . . . . . . 66

3.3.1 Operadores multilineares aproximáveis e compactos . . . . . . . 66

3.3.2 Operadores multilineares hiper-nucleares e hiper- $(s ; r)$ nucleares . . . . . . . . . . . . . . . . . . . . . . . . 68

3.3.3 Operadores multilineares hiper- $\sigma(p)$-nucleares . . . . . . . . . . 71

4 O Caso Polinomial $\quad 72$

4.1 A hiper-transformada de Borel polinomial . . . . . . . . . . . . . . . 72

4.2 Ideais de polinômios de composição . . . . . . . . . . . . . . . . . . . 83

4.3 Aplicações . . . . . . . . . . . . . . . . . . . . . . . . . . . 92 92

4.3.1 Polinômios homogêneos aproximáveis e compactos . . . . . . . . . . 92

4.3.2 Polinômios homogêneos hiper-nucleares . . . . . . . . . . . . . . . . 93

4.3.3 Polinômios homogêneos hiper- $\sigma(p)$-nucleares . . . . . . . . . . . . . 97

5 Hiper-ideais gerados por classes de sequências 105

5.1 Hiper-ideais de operadores multilineares gerados por classes de sequências . 105

5.2 Classes de sequências e ideais de polinômios . . . . . . . . . . . . . . 113

5.3 Comparação com os ideais de composição . . . . . . . . . . . . . . . . . . . 121

Referências 


\section{Introdução}

O estudo de espaços duais sempre desempenhou um importante papel no desenvolvimento da Análise Funcional e suas subáreas, como a Teoria de Operadores. Os mais conhecidos teoremas que descrevem funcionais lineares contínuos em espaços de Banach são os teoremas de representação devidos a F. Riesz, que representam funcionais lineares em espaços de funções contínuas, funções integráveis e também em espaços de sequências (veja, por exemplo, [18, 48, 53]).

Em Holomorfia em Dimensão Infinita, a representação de funcionais lineares tanto em espaços de operadores multilineares como em espaços de polinômios homogêneos, e também em espaços de funções holomorfas, tem sido uma importante ferramenta desde a década de 1960 com o aparecimento e desenvolvimento dos trabalhos de Gupta [46, 47]. Nesses trabalhos, Gupta introduziu a técnica da transformada de Borel na área. Vejamos, brevemente, uma descrição dessa técnica. Para $E_{1}, \ldots, E_{n}$ e $F$ espaços de Banach, consideremos um subespaço $\mathcal{M}\left(E_{1}, \ldots, E_{n} ; F\right)$ do espaço dos operadores multilineares de $E_{1} \times \cdots \times E_{n}$ em $F$ munido de uma norma completa $\|\cdot\|_{\mathcal{M}}$. A ideia básica da técnica é identificar um subespaço $\mathcal{R}\left(E_{1}^{*}, \ldots, E_{n}^{*} ; F^{*}\right)$ do espaço dos operadores multilineares de $E_{1}^{*} \times \cdots \times E_{n}^{*}$ em $F^{*}$, em que $E^{*}$ denota o dual topológico do espaço de Banach $E$, e uma norma completa $\|\cdot\|_{\mathcal{R}}$ tais que a transformada de Borel

$$
\begin{gathered}
\beta_{n}:\left(\mathcal{M}\left(E_{1}, \ldots, E_{n} ; F\right),\|\cdot\|_{\mathcal{M}}\right)^{*} \longrightarrow\left(\mathcal{R}\left(E_{1}^{*}, \ldots, E_{n}^{*} ; F^{*}\right),\|\cdot\|_{\mathcal{R}}\right) \\
\beta_{n}(\phi)\left(x_{1}^{*}, \ldots, x_{n}^{*}\right)(y)=\phi\left(x_{1}^{*} \otimes \cdots \otimes x_{n}^{*} \otimes y\right),
\end{gathered}
$$

seja um isomorfismo isométrico, onde $x_{1}^{*} \otimes \cdots \otimes x_{n}^{*} \otimes y: E_{1} \times \cdots \times E_{n} \longrightarrow F$ é o operador $n$-linear dado por

$$
\left(x_{1}^{*} \otimes \cdots \otimes x_{n}^{*} \otimes y\right)\left(x_{1}, \ldots, x_{n}\right)=x_{1}^{*}\left(x_{1}\right) \cdots x_{n}^{*}\left(x_{n}\right) y
$$

No caso polinomial, considera-se um subespaço $\mathcal{Q}\left({ }^{n} E ; F\right)$ do espaço dos polinômios $n$-homogêneos do espaço de Banach $E$ no espaço de Banach $F$ munido de uma norma 
completa $\|\cdot\|_{\mathcal{Q}}$ e procura-se identificar um subespaço $\mathcal{R}\left({ }^{n} E^{*} ; F^{*}\right)$ do espaço dos polinômios $n$-homogêneos de $E^{*}$ em $F^{*}$ e uma norma completa $\|\cdot\|_{\mathcal{R}}$ tais que a transformada de Borel polinomial

$$
\begin{gathered}
\beta_{n}:\left(\mathcal{Q}\left({ }^{n} E ; F\right),\|\cdot\|_{\mathcal{Q}}\right)^{*} \longrightarrow\left(\mathcal{R}\left({ }^{n} E^{*} ; F^{*}\right),\|\cdot\|_{\mathcal{R}}\right) \\
\beta_{n}(\phi)\left(x^{*}\right)(y)=\phi\left(\left[x^{*}\right]^{n} \otimes y\right),
\end{gathered}
$$

seja um isomorfismo isométrico, onde $\left[x^{*}\right]^{n} \otimes y: E \longrightarrow F$ é o polinômio $n$-homogêneo dado por

$$
\left(\left[x^{*}\right]^{n} \otimes y\right)(x)=x^{*}(x)^{n} y
$$

Com essa técnica à disposição, surgiram diversas publicações descrevendo funcionais lineares em variados espaços, o que comprova seu sucesso. Destacamos os trabalhos de:

(i) T. A. W. Dwyer em 1976, que representou funcionais lineares em espaços de operadores multilineares nucleares [37];

(ii) R. Alencar em 1992, que representou funcionais lineares em espaços de operadores multilineares e de polinômios homogêneos que podem ser aproximados por operadores de tipo finito [4];

(iii) M. Matos em 1993, que representou funcionais lineares em espaços de operadores multilineares $\left(s ; r_{1}, \ldots, r_{n}\right)$-nucleares [52];

(iv) D. Carando e V. Dimant em 2000, que melhoraram os resultados de Dwyer sobre a representação de funcionais lineares em espaços de polinômios nucleares [31].

Os livros $[35,36]$ de Dineen são excelentes fontes para resultados desse tipo e suas muitas aplicações. Entre os desenvolvimentos mais recentes, citamos os seguintes, que incluem aplicações à dinâmica linear: [15, 25, 38, 39, 40, 41, 58].

Embora muito frutífera, essa técnica possui a limitação de exigir, no caso multilinear, que o espaço $\mathcal{M}\left(E_{1}, \ldots, E_{n} ; F\right)$ esteja contido no espaço dos operadores multilineares que podem ser aproximados, na norma usual, por operadores multilineares de tipo finito. E no caso polinomial, exige que o espaço $\mathcal{Q}\left({ }^{n} E ; F\right)$ esteja contido no espaço dos polinômios que podem ser aproximados, na norma usual, por polinômios de tipo finito. Essas restrições impossibilitam, por exemplo, a representação de funcionais lineares em espaços de operadores multilineares compactos e de polinômios homogêneos compactos.

Com o objetivo de superar essas barreiras pretendemos, nesta tese, desenvolver uma nova técnica, a qual denominamos de hiper-transformada de Borel, que fornece representações de duais de muitos espaços de operadores multilineares e polinômios homogêneos que não são alcançados pela técnica padrão. É importante destacar que as duas técnicas 
são independentes entre si, o que significa que uma representação obtida usando a transformada de Borel clássica não pode ser obtida usando a hiper-transformada de Borel e vice-versa. Isso decorre do fato de que a transformada de Borel funciona apenas em espaços de operadores multilineares/polinômios homogêneos nos quais os operadores multilineares/polinômios homogêneos de tipo finito são densos, ao passo que a hiper-transformada de Borel, por nós aqui introduzida e desenvolvida, funciona em espaços de operadores multilineares/polinômios homogêneos nos quais os operadores/polinômios de posto finito são densos. Como muitos espaços de operadores multilineares/polinômios estão contidos nos operadores multilineares/polinômios de posto finito mas não estão contidos nos operadores/polinômios de tipo finito, justifica-se uma nova técnica para representar funcionais lineares nesses espaços.

No caso linear, a transformada de Borel funciona bem para representar funcionais lineares em ideais de operadores, pois ideais de operadores contêm os operadores de posto finito. Da mesma forma, as transformadas de Borel multilinear e polinomial funcionam bem para representar funcionais lineares em ideais de operadores multilineares (multiideais) e em ideais de polinômios, pois os multi-ideais contêm os operadores de tipo finito e os ideais de polinômios contêm os polinômios de tipo finito. Já os hiper-ideais de operadores multilineares e de polinômios homogêneos (veja [20, 21, 72]) contêm os operadores multilineares e os polinômios de posto finito, o que faz com que esses ideais sejam os objetos de aplicação das hiper-transformadas de Borel multilinear e polinomial.

Descrevemos a seguir como estruturamos a tese para alcançar o objetivo proposto. Destinamos o primeiro capítulo para apresentar conceitos e resultados básicos que serão necessários para o desenvolvimento da tese. Optamos também por inserir alguns exemplos e resultados mais específicos sobre representação de duais de determinadas classes de operadores lineares e multilineares que nos serão úteis. Como a linearização de operadores multilineares e de polinômios homogêneos e hiper-ideais de composição são dois tópicos imprescindíveis para os nossos principais resultados, ambos receberam destaque neste capítulo.

As classes de operadores nucleares e suas variantes sempre aparecem desempenhando um papel de destaque nas teorias linear, multilinear e polinomial. Em particular, as transformadas de Borel linear, multilinear e polinomial funcionam bem em classes de operadores do tipo nuclear. É natural então buscar classes relacionadas para as quais a hiper-transformada de Borel funcione bem. Para o caso dos operadores $\left(s ; r_{1}, \ldots, r_{n}\right)$ nucleares, essa nova classe foi introduzida e estudada em [20,72] sob o nome de operadores hiper- $(s ; r)$-nucleares. Provaremos no Capítulo 2 alguns resultados que prepararão essa 
classe para a aplicação da hiper-transformada de Borel. Mas a classe relacionada aos operadores $\sigma(p)$-nucleares de [15] ainda não havia sido estudada. Introduzimos então, também no Capítulo 2, a classe dos operadores multilineares hiper- $\sigma(p)$-nucleares, e também provamos resultados preparatórios para a aplicação da hiper-transformada de Borel.

No Capítulo 3 desenvolvemos o tema central da tese, a saber, a hiper-transformada de Borel. Dado um subespaço vetorial $\mathcal{H}\left(E_{1}, \ldots, E_{n} ; F\right)$ do espaço dos operadores $n$-lineares contínuos de $E_{1} \times \cdots \times E_{n}$ em $F$ munido de uma norma completa $\|\cdot\|_{\mathcal{H}}$ e contendo os operadores multilineares de posto finito, a hiper-transformada de Borel é o operador

$$
\mathcal{B}_{n}:\left(\mathcal{H}\left(E_{1}, \ldots, E_{n} ; F\right),\|\cdot\|_{\mathcal{H}}\right)^{*} \longrightarrow \mathcal{L}\left(\mathcal{L}\left(E_{1}, \ldots, E_{n}\right) ; F^{*}\right), \mathcal{B}_{n}(\phi)(A)(y)=\phi(A \otimes y),
$$

onde $A \otimes y\left(x_{1}, \ldots, x_{n}\right)=A\left(x_{1}, \ldots, x_{n}\right) y$. Provamos primeiramente a boa definição e as propriedades básicas de $\mathcal{B}_{n}$. Em seguida mostramos que a única classe $\mathcal{H}$ para a qual $\mathcal{B}_{n}$ é um isomorfismo isométrico sobre sua imagem é a classe dos operadores multilineares hiper-nucleares.

Passamos então a investigar o caso em $\mathcal{H}$ não é o hiper-ideal dos operadores multilineares hiper-nucleares. Para isso é preciso substituir a norma em $\mathcal{B}_{n}\left(\mathcal{H}\left(E_{1}, \ldots, E_{n} ; F\right)^{*}\right) \subseteq$ $\mathcal{L}\left(\mathcal{L}\left(E_{1}, \ldots, E_{n}\right) ; F^{*}\right)$ por outra norma e identificar o subespaço sobre o qual $\mathcal{B}_{n}$ seja um isomorfismo isométrico. Para isso introduziremos uma noção mais geral que ideal de Banach de operadores, a qual chamamos de semi-ideal de operadores à esquerda.

O principal resultado geral do terceiro capítulo estabelece condições para que as seguintes afirmações sejam equivalentes para um ideal de operadores $\mathcal{I}$ (o símbolo $\mathcal{I} \circ \mathcal{L}$ denota o respectivo ideal de composição de operadores multilineares):

(i) A transformada de Borel linear $\beta$ representa funcionais lineares em $\mathcal{I}(E ; F)$.

(ii) A hiper-transformada de Borel $\mathcal{B}_{n}$ representa funcionais lineares no hiper-ideal de composição de operadores multilineares $\mathcal{I} \circ \mathcal{L}\left(E_{1}, \ldots, E_{n} ; F\right)$ para todo $n$.

(iii) A hiper-transformada de Borel $\mathcal{B}_{n}$ representa funcionais lineares no hiper-ideal de composição de operadores multilineares $\mathcal{I} \circ \mathcal{L}\left(E_{1}, \ldots, E_{n} ; F\right)$ para algum $n$.

Para fins práticos, isso ensina como utilizar uma representação de funcionais lineares em espaços de operadores lineares por meio da transformada de Borel para representar funcionais lineares em espaços de operadores multilineares. Concluiremos esse terceiro capítulo apresentando aplicações concretas da hiper-transformada de Borel multilinear. Representaremos duais de classes de operadores já bastante estudadas, como os operadores multilineares aproximáveis, compactos, hiper-nucleares, hiper- $(s ; r)$-nucleares; e também da nova classe dos operadores multilineares hiper- $\sigma(p)$-nucleares. Ressaltamos que, até onde sabemos, esses são os primeiros teoremas de representação de funcionais lineares 
nessas classes de operadores.

No Capítulo 4 desenvolveremos a contrapartida polinomial da teoria elaborada nos Capítulos 2 e 3. Começamos definindo a hiper-transformada de Borel polinomial e desenvolvendo sua teoria básica. Como de costume, alguns argumentos da teoria multilinear funcionam bem para o caso polinomial e outros não. Nesse capítulo passaremos pelas duas situações. Por um lado, boa parte da teoria da hiper-transformada de Borel multilinear funciona da mesma forma no caso polinomial. Por outro lado, veremos que no caso polinomial não funcionam as equivalências enunciadas acima para o caso multilinear. Mais precisamente, não sabemos se, no caso polinomial, vale a implicação (iii) $\Rightarrow$ (i), e daremos razões para acreditar que não deve ser verdadeiro. Trataremos dessas diferenças com detalhes e provaremos um resultado parcial nesta direção. Entendemos que esse fenômeno evidencia mais uma importante diferença entre as teorias multilinear e polinomial, deixando claro que elas não são idênticas e que, portanto, ambas merecem ser estudadas. Como a implicação (i) $\Rightarrow$ (ii), que é a mais útil para fins práticos, continua válida no contexto dos polinômios homogêneos, finalizaremos o capítulo apresentando algumas aplicações concretas da hiper-transformada de Borel polinomial na representação de funcionais lineares em vários espaços de polinômios.

Está claro neste ponto que os hiper-ideais de operadores multilineares e de polinômios homogêneos desempenham papel crucial nesta tese. Apesar de muitos exemplos serem conhecidos e muitas técnicas para gerar hiper-ideais tenham sido desenvolvidas, identificamos uma técnica que ainda não havia sido estudada e que gera novos hiper-ideais de operadores multilineares e de polinômios homogêneos. Essa nova técnica é baseada no conceito de classes de sequências, introduzido em [11] e desenvolvido e aplicado em, por exemplo, [1, 10, 12, 13, 14, 30, 64, 65]. Após provar os resultados básicos da nova técnica, mostraremos que muitos multi-ideais e ideais de polinômios já bem conhecidos e estudados são, na verdade, hiper-ideais ou ideais bilaterais. Na última seção desse Capítulo 5 mostraremos que os hiper-ideais gerados por esta nova técnica não são ideais de composição, o que comprova que estamos de fato gerando novos hiper-ideais de operadores multilineares e novos hiper-ideais/ideais bilaterais de polinômios homogêneos.

Os resultados provados nos Capítulos 2 e 3 estão publicados no seguinte artigo:

- G. Botelho, R. Wood, On the representation of linear functionals on hyper-ideals of multilinear operators, Banach J. Math. Anal. 15 (2021), no. 1, Paper No. 25, 23 pp.

Os resultados provados no Capítulo 4 estão aceitos para publicação no seguinte artigo: - G. Botelho, R. Wood, The polynomial hyper-Borel transform, Bull. Braz. Math. Soc., 
to appear (https://link.springer.com/article/10.1007/s00574-021-00268-3).

Os resultados provados no Capítulo 5 estão submetidos para publicação no seguinte artigo:

- G. Botelho, R. Wood, Hyper-ideals of multilinear operators and two-sided polynomial ideals generated by sequence classes, disponível em arXiv:2110.00051v1[math.FA], 2021. 


\section{Capítulo 1}

\section{Preliminares}

Neste capítulo vamos apresentar resultados básicos e conceitos fundamentais no desenvolvimento desta tese. Por se tratarem de resultados conhecidos, não apresentaremos as demonstrações, apenas referências serão fornecidas. Usaremos a notação e a terminologia usuais da Análise Funcional Linear e da Teoria dos Espaços de Banach (veja [18, 50, 53]).

Denotaremos por $\mathbb{K}$ o corpo dos números reais $\mathbb{R}$ ou complexos $\mathbb{C}$. Os espaços vetoriais considerados a seguir serão sempre sobre $\mathbb{K}$. Seja $E$ um espaço vetorial sobre $\mathbb{K}$. Uma função $\|\cdot\|: E \longrightarrow[0, \infty)$ é chamada uma norma se satisfaz:

(1) $\|x\|=0$ se, e somente se, $x=0$.

(2) $\|\lambda x\|=|\lambda| \cdot\|x\|$ para todos $\lambda \in \mathbb{K}$ e $x \in E$.

(3) $\|x+y\| \leq\|x\|+\|y\|$ para quaisquer $x, y \in E$.

Denomina-se de espaço normado o par $(E,\|\cdot\|)$. Considerando a métrica induzida pela norma, a saber,

$$
(x, y) \in E \times E \mapsto\|x-y\| \in \mathbb{R},
$$

obtém-se uma estrutura de espaço métrico para $E$. Se $E$ for completo com respeito a essa norma, o par $(E,\|\cdot\|)$ é chamado de espaço de Banach. Para maiores detalhes sobre a teoria dos espaços de Banach referimos [53].

A seguir definimos alguns espaços de sequências que são espaços de Banach.

Definição 1.0.1. Sejam $(E,\|\cdot\|)$ um espaço de Banach e $p \geq 1$. Denotaremos por $\ell_{p}(E)$ 
o espaço das sequências $\left(x_{j}\right)_{j=1}^{\infty}$ em $E$ tais que

$$
\sum_{j=1}^{\infty}\left\|x_{j}\right\|^{p}<\infty
$$

chamadas sequências absolutamente p-somáveis. A expressão

$$
\left\|\left(x_{j}\right)_{j=1}^{\infty}\right\|_{p}=\left(\sum_{j=1}^{\infty}\left\|x_{j}\right\|^{p}\right)^{\frac{1}{p}}
$$

define uma norma em $\ell_{p}(E)$ tornando-o um espaço de Banach. Denotaremos $\ell_{p}(\mathbb{K})$ por $\ell_{p}$

Por $E^{*}$ denotamos o dual topológico do espaço $E$ e por $B_{E}$ a bola unitária fechada de $E$, isto é, $B_{E}=\{x \in E:\|x\| \leq 1\}$.

Definição 1.0.2. Sejam $(E,\|\cdot\|)$ um espaço de Banach e $p \geq 1$. Denotaremos por $\ell_{p}^{w}(E)$ o espaço das sequências $\left(x_{j}\right)_{j=1}^{\infty}$ em $E$ tais que

$$
\sum_{j=1}^{\infty}\left|\varphi\left(x_{j}\right)\right|^{p}<\infty \text { para todo } \varphi \in E^{*},
$$

chamadas sequências fracamente p-somáveis. A expressão

$$
\left\|\left(x_{j}\right)_{j=1}^{\infty}\right\|_{w, p}=\sup _{\varphi \in B_{E^{*}}}\left(\sum_{j=1}^{\infty}\left|\varphi\left(x_{j}\right)\right|^{p}\right)^{\frac{1}{p}}
$$

define uma norma em $\ell_{p}(E)$ que o torna um espaço de Banach. É imediato que $\ell_{p}^{w}(\mathbb{K})=\ell_{p}$. Definição 1.0.3. Denotaremos o espaço das sequências limitadas de $E$ por $\ell_{\infty}(E)$ e definimos a norma

$$
\left\|\left(x_{j}\right)_{j=1}^{\infty}\right\|_{\infty}=\sup _{j \in \mathbb{N}}\left\|x_{j}\right\|
$$

que torna $\ell_{\infty}(E)$ um espaço de Banach.

Definição 1.0.4. Denotamos por $c_{0}(E)$ o subespaço fechado de $\ell_{\infty}(E)$ formado pelas sequências que convergem para zero em norma, ou seja,

$$
c_{0}(E):=\left\{\left(x_{j}\right)_{j=1}^{\infty}: x_{j} \in E \text { e } x_{j} \longrightarrow 0\right\},
$$

o qual se torna um espaço de Banach com a norma $\|\cdot\|_{\infty}$. 
Definimos ainda o subespaço vetorial de $c_{0}(E)$ formado pelas sequências eventualmente nulas:

$$
c_{00}(E):=\left\{\left(x_{j}\right)_{j=1}^{\infty} \in c_{0}(E): \text { existe } j_{0} \in \mathbb{N} \text { tal que } x_{j}=0 \text { para todo } j \geq j_{0}\right\} .
$$

Vale notar que $c_{00}(E)$ é um espaço normado que não é de Banach.

Proposição 1.0.5. (Desigualdade de Hölder) [18, Proposição 1.4.1] Sejam $p, q \in[1, \infty)$ tais que $\frac{1}{p}+\frac{1}{q}=1$. Se $\left(a_{j}\right)_{j=1}^{\infty} \in \ell_{p} e\left(b_{j}\right)_{j=1}^{\infty} \in \ell_{q}$, então

$$
\sum_{j=1}^{\infty}\left|a_{j}\right| \cdot\left|b_{j}\right| \leq\left(\sum_{j=1}^{\infty}\left|a_{j}\right|^{p}\right)^{\frac{1}{p}}+\left(\sum_{j=1}^{\infty}\left|b_{j}\right|^{q}\right)^{\frac{1}{q}}
$$

\subsection{Operadores lineares contínuos}

Denotaremos o conjunto de todos os operadores lineares contínuos do espaço de Banach $E$ no espaço de Banach $F$ por $\mathcal{L}(E ; F)$, que se torna um espaço de Banach com a norma usual de operadores: para todo $u \in \mathcal{L}(E ; F)$,

$$
\|u\|=\sup _{x \in B_{E}}\|u(x)\|=\inf \{C \geq 0:\|u(x)\| \leq C \cdot\|x\| \text { para todo } x \in E\} .
$$

Conforme fizemos antes, no caso em que $F=\mathbb{K}$ denotaremos $\mathcal{L}(E ; \mathbb{K})$ por $E^{*}$ e o denominamos de dual topológico de $E$. Os elementos de $E^{*}$ são chamados de funcionais lineares.

Dados $\varphi \in E^{*}$ e $y \in F$, consideramos o operador linear contínuo

$$
\varphi \otimes y: E \longrightarrow F,(\varphi \otimes y)(x)=\varphi(x) y .
$$

É imediato que $\|\varphi \otimes y\|=\|\varphi\| \cdot\|y\|$.

Definição 1.1.1. [59, B.1.3] Diz-se que um operador $u \in \mathcal{L}(E ; F)$ tem posto finito se sua imagem $u(E)$ é um subespaço de $F$ de dimensão finita, ou, equivalentemente, se existem $n \in \mathbb{N}, \varphi_{j} \in E^{*}$ e $y_{j} \in F, j=1, \ldots, n$ tais que

$$
u=\sum_{j=1}^{n} \varphi_{j} \otimes y_{j}
$$

Denotaremos o subespaço dos operadores lineares de posto finito de $E$ em $F$ por $\mathcal{F}(E ; F)$. 
Definição 1.1.2. [50, Definition 1.e.1] Um espaço de Banach E possui a propriedade da aproximação se para todo subconjunto compacto $K$ de $E$ e todo $\varepsilon>0$ existe um operador de posto finito $u \in \mathcal{F}(E ; E)$ tal que

$$
\|x-u(x)\| \leq \varepsilon \text { para todo } x \in K .
$$

Definição 1.1.3. [50, Definition 1.e.11] Um espaço de Banach $E$ possui a propriedade da aproximação limitada se existe $\lambda \geq 1$ tal que para todo subconjunto compacto $K$ de $E$ e todo $\varepsilon>0$ existe um operador de posto finito $u \in \mathcal{F}(E ; E)$ tal que $\|u\| \leq \lambda$ e

$$
\|x-u(x)\| \leq \varepsilon \text { para todo } x \in K .
$$

Definição 1.1.4. Sejam $E$ e $F$ espaços de Banach. Um operador bijetor $u \in \mathcal{L}(E ; F)$ é chamado de isomorfismo, e neste caso os espaços $E$ e $F$ são isomorfos. Lembre-se que o operador inverso $u^{-1}: F \longrightarrow E$ é contínuo pelo Teorema da Aplicação Aberta (Teorema 1.1 .12 a seguir). Se, além disso, $\|u(x)\|=\|x\|$ para todo $x \in E$, $u$ é chamado de isomorfismo isométrico, e neste caso $E$ e $F$ são isomorfos isometricamente.

Definição 1.1.5. [18, Definição 4.3.9] Sejam $E$ e $F$ espaços vetoriais normados e $u \in$ $\mathcal{L}(E, F)$ um operador linear contínuo. Definimos o operador $u^{*}: F^{*} \longrightarrow E^{*}$ por

$$
u^{*}(\varphi)(x)=\varphi(u(x)) \text { para todos } x \in E \text { e } \varphi \in F^{*} .
$$

O operador $u^{*}$ é chamado adjunto de $u$.

Proposição 1.1.6. [18, Proposição 4.3.11] Seja $u \in \mathcal{L}(E ; F)$. Então $u^{*} \in \mathcal{L}\left(F^{*}, E^{*}\right)$ e $\left\|u^{*}\right\|=\|u\|$. Mais ainda, se u é um isomorfismo (isométrico), então $u^{*}$ também é um isomorfismo (isométrico).

Teorema 1.1.7. [32, Theorem 2.1] Sejam E e $F$ espaços de Banach e $u \in \mathcal{L}(E ; F)$. Então:

(i) u é sobrejetor se, e somente se, $u^{*}$ é um isomorfismo sobre sua imagem.

(ii) u é um isomorfismo sobre sua imagem se, e somente se, $u^{*}$ é sobrejetor.

Proposição 1.1.8. [18, Exercício 3.6.29] Um subespaço vetorial $F$ do espaço normado $E$ é denso em $E$ se, e somente se, o único funcional $\varphi \in E^{*}$ que se anula em $F$ é o funcional nulo.

Proposição 1.1.9. [33, p. 92] Sejam E e F espaços de Banach, $1 \leq p \leq \infty$ e u $\in \mathcal{L}(E ; F)$. (i) $S e\left(x_{j}\right)_{j=1}^{\infty} \in \ell_{p}(E)$, então

$$
\left(u\left(x_{j}\right)\right)_{j=1}^{\infty} \in \ell_{p}(F) e\left\|\left(u\left(x_{j}\right)\right)_{j=1}^{\infty}\right\|_{p} \leq\|u\| \cdot\left\|\left(x_{j}\right)_{j=1}^{\infty}\right\|_{p} .
$$


(ii) $S e\left(x_{j}\right)_{j=1}^{\infty} \in \ell_{p}^{w}(E)$, então

$$
\left(u\left(x_{j}\right)\right)_{j=1}^{\infty} \in \ell_{p}^{w}(F) e\left\|\left(u\left(x_{j}\right)\right)_{j=1}^{\infty}\right\|_{w, p} \leq\|u\| \cdot\left\|\left(x_{j}\right)_{j=1}^{\infty}\right\|_{w, p} .
$$

Diz-se que um operador $u \in \mathcal{L}(E ; F)$ é compacto se $u\left(B_{E}\right)$ é um subconjunto relativamente compacto de $F$ (veja [18, Seção 7.2]). O subespaço de $\mathcal{L}(E ; F)$ formado pelos operadores compactos é denotado por $\mathcal{K}(E ; F)$.

Proposição 1.1.10. [51, Proposition 1.3] Sejam E e F espaços de Banach. Se E* ou F tem a propriedade da aproximação, então

$$
\overline{\mathcal{F}(E ; F)}=\mathcal{K}(E ; F) .
$$

Proposição 1.1.11. [18, Proposição 4.3.1] Para todo espaço normado E, o operador linear

$$
J_{E}: E \longrightarrow E^{* *}, J_{E}(x)(\varphi)=\varphi(x)
$$

para todos $x \in E$ e $\varphi \in E^{*}$, é uma imersão isométrica linear, isto é, J $J_{E}$ é linear e $\left\|J_{E}(x)\right\|=\|x\|$ para todo $x \in E$. O operador $J_{E}$ é chamado de mergulho canônico de $E$ em $E^{* *}$.

Em particular, o subespaço $J_{E}(E)$ de $E^{* *}$ é isomorfo isometricamente a $E$.

Teorema 1.1.12. (Teorema da Aplicação Aberta) [18, Teorema 2.4.2] Sejam E e F espaços de Banach e $u \in \mathcal{L}(E ; F)$ sobrejetor. Então, u é uma aplicação aberta, isto é, $u(V)$ é aberto em $F$, sempre que $V$ for aberto em $E$.

Teorema 1.1.13. (Teorema de Hahn-Banach) [18, Corolário 3.1.5] Sejam E um espaço normado, $E \neq\{0\}$, e $x \in E$. Então,

$$
\|x\|=\sup \left\{|\varphi(x)|: \varphi \in E^{*} e\|\varphi\| \leq 1\right\} .
$$

Para a definição de subespaço complementado de um espaço de Banach, veja [18, Definição 3.3.3]. O resultado a seguir é bem conhecido, mas optamos por demonstrálo pois não o encontramos na forma exata em que nos será útil. O mais próximo que encontramos foi [59, Lemma B.4.4].

Lema 1.1.14. Sejam $E$ e $F$ espaços de Banach para os quais existem operadores $u \in$ $\mathcal{L}(F ; E)$ e $v \in \mathcal{L}(E ; F)$ tais que $v \circ u=i d_{F}$. Então $u(F)$ é subespaço complementado de E e u: $F \longrightarrow u(F)$ é um isomorfismo cujo isomorfismo inverso é a restrição de $v$ a $u(F)$. 
Demonstração. Vejamos que $u \circ v: E \longrightarrow E$ é uma projeção sobre $u(F)$. Para todo $x \in E$,

$$
\begin{aligned}
(u \circ v)^{2}(x) & =(u \circ v \circ u \circ v)(x)=u(v(u(v(x))))=u((v \circ u)(v(x)))=u\left(i d_{F}(v(x))\right) \\
& =u(v(x))=(u \circ v)(x),
\end{aligned}
$$

provando que $u \circ v$ é projeção. Devemos provar agora que $(u \circ v)(E)=u(F)$. É claro que $(u \circ v)(E)=u(v(E)) \subseteq u(F)$. Para a inclusão inversa, veja que:

$x \in u(F) \Longrightarrow$ existe $y \in F$ tal que $x=u(y) \Longrightarrow x=u\left(i d_{F}(y)\right) \Longrightarrow x=u((v \circ u)(y))$ $\Longrightarrow x=(u \circ v)(u(y)) \Longrightarrow x \in(u \circ v)(E)$.

Está provado que $u(F)$ é subespaço complementado de $E$.

Chamemos de $\hat{v}: u(F) \longrightarrow F$ a restrição de $v$ a $u(F)$. Para todo $y \in F$,

$$
(\hat{v} \circ u)(y)=\hat{v}(u(y))=v(u(y))=(v \circ u)(y)=i d_{F}(y)=y
$$

E para todo $x \in u(F)$ existe $y \in F$ tal que $x=u(y)$, e portanto

$$
(u \circ \hat{v})(x)=u(\hat{v}(x))=u(v(x))=u(v(u(y)))=u((v \circ u)(y))=u(y)=x .
$$

Isso prova que $u: F \longrightarrow u(F)$ é bijetora e que sua inversa é $\hat{v}$. Por ser a restrição de um operador contínuo, $\hat{v}$ também é contínuo, e portanto $u: F \longrightarrow u(F)$ é um isomorfismo, com inversa $\hat{v}$.

\subsection{Operadores multilineares contínuos}

Nesta seção compilamos as definições e propriedades básicas sobre operadores multilineares que serão necessárias para o desenvolvimento da tese. Para mais detalhes e informações adicionais referimos os livros de Mujica [57] e de Dineen [36].

Definição 1.2.1. Sejam $X_{1}, \ldots, X_{n}$ e $Y$ espaços vetoriais. Dizemos que um operador $A: X_{1} \times \cdots \times X_{n} \longrightarrow Y$ é multilinear ou $n$-linear se para todo $k=1, \ldots, n$, todos $x_{1} \in X_{1}, \ldots, x_{k-1} \in X_{k-1}, x_{k}, y_{k} \in X_{k}, x_{k+1} \in X_{k+1}, \ldots, x_{n} \in X_{n}$, e $\lambda \in \mathbb{K}$,

$$
A\left(x_{1}, \ldots, x_{k}+\lambda y_{k}, \ldots, x_{n}\right)=A\left(x_{1}, \ldots, x_{k}, \ldots, x_{n}\right)+\lambda A\left(x_{1}, \ldots, y_{k}, \ldots, x_{n}\right) .
$$

Denotamos o espaço desses operadores por $L\left(X_{1}, \ldots, X_{n} ; Y\right)$, o qual se torna um espaço vetorial com as operações usuais de funções (operações pontuais). 
Quando $E_{1}, \ldots, E_{n}$ e $F$ são espaços normados, denotamos por $\mathcal{L}\left(E_{1}, \ldots, E_{n} ; F\right)$ o subespaço de $L\left(E_{1}, \ldots, E_{n} ; F\right)$ dos operadores multilineares contínuos. Se $E_{1}=\cdots=$ $E_{n}=E$, escrevemos $L\left({ }^{n} E ; F\right)$ e $\mathcal{L}\left({ }^{n} E ; F\right)$, respectivamente.

E quando $F=\mathbb{K}$, as notações utilizadas para os espaços acima serão simplesmente $L\left(E_{1}, \ldots, E_{n}\right), L\left({ }^{n} E\right), \mathcal{L}\left(E_{1}, \ldots, E_{n}\right)$ e $\mathcal{L}\left({ }^{n} E\right)$. Chamamos os elementos de $\mathcal{L}\left(E_{1}, \ldots, E_{n}\right)$ de formas multilineares.

A proposição a seguir nos fornece, entre outras coisas, algumas caracterizações de operadores multilineares contínuos.

Proposição 1.2.2. [71, Proposições 2.7, 2.8, 2.10 e 2.11] Sejam $E_{1}, \ldots, E_{n}, F$ espaços normados e $A \in \mathcal{L}\left(E_{1}, \ldots, E_{n} ; F\right)$.

(i) As seguintes afirmações são equivalentes:

(a) A é contínuo.

(b) A é contínuo na origem.

(c) Existe uma constante $C \geq 0$ tal que

$$
\left\|A\left(x_{1}, \ldots, x_{n}\right)\right\| \leq C\left\|x_{1}\right\| \cdots\left\|x_{n}\right\|
$$

para todos $x_{i} \in E_{i}, i=1, \ldots, n$.

(d) $\sup \left\{\left\|A\left(x_{1}, \ldots, x_{n}\right)\right\|: x_{i} \in B_{E_{i}}, i=1, \ldots, n\right\}<\infty$.

(ii) A função $A \mapsto\|A\|:=\sup \left\{\left\|A\left(x_{1}, \ldots, x_{n}\right)\right\|: x_{i} \in B_{E_{i}}, i=1, \ldots, n\right\}$ define uma norma em $\mathcal{L}\left(E_{1}, \ldots, E_{n} ; F\right)$.

(iii) Se $F$ é um espaço de Banach, então $\mathcal{L}\left(E_{1}, \ldots, E_{n} ; F\right)$ munido da norma definida em (ii) também é espaço de Banach.

Nos referiremos à norma definida no item (ii) como norma usual.

Definimos a seguir duas classes de operadores multilineares que serão especialmente importantes no que segue.

Definição 1.2.3. Sejam $E_{1}, \ldots, E_{n}$ e $F$ espaços de Banach. Dados $\varphi^{(1)} \in E_{1}^{*}, \ldots, \varphi^{(n)} \in$ $E_{n}^{*}$ e $y \in F$, consideramos o operador $n$-linear contínuo $\varphi^{(1)} \otimes \cdots \otimes \varphi^{(n)} \otimes y \in \mathcal{L}\left(E_{1}, \ldots, E_{n} ; F\right)$ definido por

$$
\left(\varphi^{(1)} \otimes \cdots \otimes \varphi^{(n)} \otimes y\right)\left(x_{1}, \ldots, x_{n}\right)=\varphi^{(1)}\left(x_{1}\right) \cdots \varphi^{(n)}\left(x_{n}\right) y
$$


É imediato que $\left\|\varphi^{(1)} \otimes \cdots \otimes \varphi^{(n)} \otimes y\right\|=\left\|\varphi^{(1)}\right\| \cdots\left\|\varphi^{(n)}\right\| \cdot\|y\|$.

Combinações lineares de operadores dessa forma são chamados de operadores $n$-lineares de tipo finito. E o subespaço de $\mathcal{L}\left(E_{1}, \ldots, E_{n} ; F\right)$ formado pelos operadores de tipo finito é denotado por $\mathcal{L}_{f}\left(E_{1}, \ldots, E_{n} ; F\right)$.

Definição 1.2.4. Sejam $E_{1}, \ldots, E_{n}$ e $F$ espaços de Banach. Diremos que um operador $n$-linear $A \in \mathcal{L}\left(E_{1}, \ldots, E_{n} ; F\right)$ é de posto finito se a dimensão do subespaço vetorial de $F$ gerado pela imagem de $A$ tem dimensão finita. Denotaremos o subespaço dos operadores $n$-lineares de posto finito por $\mathcal{L}_{\mathcal{F}}\left(E_{1}, \ldots, E_{n} ; F\right)$.

Dados $B \in \mathcal{L}\left(E_{1}, \ldots, E_{n}\right)$ e $y \in F$, consideramos o operador $n$-linear contínuo $B \otimes y \in$ $\mathcal{L}\left(E_{1}, \ldots, E_{n} ; F\right)$ definido por

$$
(B \otimes y)\left(x_{1}, \ldots, x_{n}\right)=B\left(x_{1}, \ldots, x_{n}\right) y
$$

É imediato que $\|B \otimes y\|=\|B\| \cdot\|y\|$.

A seguinte caracterização dos operadores multilineares de posto finito é bastante útil:

Proposição 1.2.5. [61, Proposição 4.2.1] Sejam $E_{1}, \ldots, E_{n}$ e F espaços de Banach. Um operador n-linear contínuo $A: E_{1} \times \cdots \times E_{n} \longrightarrow F$ é de posto finito se, e somente se, existem $k \in \mathbb{N}, B_{j} \in \mathcal{L}\left(E_{1}, \ldots, E_{n}\right)$ e $y_{j} \in F$, para $j=1, \ldots, k$, tais que

$$
A\left(x_{1}, \ldots, x_{n}\right)=\sum_{j=1}^{k}\left(B_{j} \otimes y_{j}\right)\left(x_{1}, \ldots, x_{n}\right)
$$

para todo $\left(x_{1}, \ldots, x_{n}\right) \in E_{1} \times \cdots \times E_{n}$. Ou seja, $\mathcal{L}_{\mathcal{F}}\left(E_{1}, \ldots, E_{n} ; F\right)$ é o subespaço gerado pelos operadores da forma $B \otimes y$.

Proposição 1.2.6. [71, Proposição 2.12] Sejam $X_{1}, \ldots, X_{m+n}, Y$ espaços vetoriais com $m, n \in \mathbb{N}$.

(a) O operador

$$
\begin{gathered}
T: L\left(X_{1}, \ldots, X_{m+n} ; Y\right) \longrightarrow L\left(X_{1}, \ldots, X_{m} ; L\left(X_{m+1}, \ldots, X_{m+n} ; Y\right)\right) \\
T(A)\left(x_{1}, \ldots, x_{m}\right)\left(x_{m+1}, \ldots, x_{m+n}\right)=A\left(x_{1}, \ldots, x_{m+n}\right)
\end{gathered}
$$

é um isomorfismo de espaços vetoriais.

(b) Se $X_{1}, \ldots, X_{m+n}, Y$ são espaços normados, então o isomorfismo do item (a) induz um isomorfismo isométrico entre $\mathcal{L}\left(X_{1}, \ldots, X_{m+n} ; Y\right)$ e $\mathcal{L}\left(X_{1}, \ldots, X_{m} ; \mathcal{L}\left(X_{m+1}, \ldots, X_{m+n} ; Y\right)\right)$. 
Observação 1.2.7. Se $E$ e $F$ são espaços de Banach, decorre do item (b) da proposição anterior que

$$
\mathcal{L}\left(E^{*}, F ; \mathbb{K}\right) \text { e } \mathcal{L}\left(E^{*} ; F^{*}\right)
$$

são isometricamente isomorfos.

Uma técnica que será frequentemente utilizada nas demonstrações ao longo do texto é a de linearização de operadores multilineares. Por isso, recordaremos agora a definição e os principais resultados envolvendo o produto tensorial. Sugerimos como referência [67] e [71].

Definição 1.2.8. Sejam $X_{1}, \ldots, X_{n}$ espaços vetoriais. O produto tensorial de $X_{1}, \ldots, X_{n}$, o qual denotaremos por $X_{1} \otimes \cdots \otimes X_{n}$, é o subespaço do dual algébrico de $L\left(X_{1}, \ldots, X_{n}\right)$ gerado pelos funcionais lineares da forma

$$
x_{1} \otimes \cdots \otimes x_{n}: L\left(X_{1}, \ldots, X_{n}\right) \longrightarrow \mathbb{K}, x_{1} \otimes \cdots \otimes x_{n}(B)=B\left(x_{1}, \ldots, x_{n}\right),
$$

onde $x_{i} \in X_{i}, i=1, \ldots, n$. Os elementos de $X_{1} \otimes \cdots \otimes X_{n}$ são chamados de tensores e seus vetores geradores são chamados de tensores elementares.

Todo tensor $x \in X_{1} \otimes \cdots \otimes X_{n}$ é uma combinação linear de tensores elementares, isto é,

$$
x=\sum_{j=1}^{k} \lambda_{j} x_{j}^{1} \otimes \cdots \otimes x_{j}^{n},
$$

$\operatorname{com} \lambda_{j} \in \mathbb{K}$ e $x_{j}^{i} \in X_{i}$, para $j=1, \ldots, k$. Neste caso dizemos que $\sum_{j=1}^{k} \lambda_{j} x_{j}^{1} \otimes \cdots \otimes x_{j}^{n}$ é uma representação de $x$.

Proposição 1.2.9. [71, Proposição 1.6] Sejam $X_{1}, \ldots, X_{n}$ espaços vetoriais. Então, para todo $i=1, \ldots, n$, e quaisquer $x_{1} \in X_{1}, \ldots, x_{i}, x_{i}^{\prime} \in X_{i}, \ldots, x_{n} \in X_{n}$, valem:

(a) $x_{1} \otimes \cdots \otimes\left(x_{i}+x_{i}^{\prime}\right) \otimes \cdots \otimes x_{n}=\left(x_{1} \otimes \cdots \otimes x_{i} \otimes \cdots \otimes x_{n}\right)+\left(x_{1} \otimes \cdots \otimes x_{i}^{\prime} \otimes \cdots \otimes x_{n}\right)$.

(b) $\lambda\left(x_{1} \otimes \cdots \otimes x_{i} \otimes \cdots \otimes x_{n}\right)=x_{1} \otimes \cdots \otimes\left(\lambda x_{i}\right) \otimes \cdots \otimes x_{n}$.

(c) $x_{1} \otimes \cdots \otimes 0 \otimes \cdots \otimes x_{n}=0$.

Observação 1.2.10. (i) Segue da homogeneidade do produto tensorial (Proposição 1.2.9(b)) que todo tensor $x$ em $X_{1} \otimes \cdots \otimes X_{n}$ tem uma representação da forma

$$
\sum_{j=1}^{k} x_{j}^{1} \otimes \cdots \otimes x_{j}^{n},
$$


$\operatorname{com} x_{j}^{i} \in X_{i}, j=1, \ldots, k$.

(ii) Segue da Proposição 1.2.9(b) que, sendo $X_{1}, \ldots, X_{n}$ espaços vetoriais, o operador

$$
\sigma_{n}: X_{1} \times \cdots \times X_{n} \longrightarrow X_{1} \otimes \cdots \otimes X_{n}, \sigma_{n}\left(x_{1}, \ldots, x_{n}\right)=x_{1} \otimes \cdots \otimes x_{n},
$$

é $n$-linear.

O resultado a seguir é conhecido como propriedade universal do produto tensorial.

Teorema 1.2.11. [67, Theorem 1.10] Para todo operador $n$-linear $A \in L\left(X_{1}, \ldots, X_{n} ; Y\right)$ existe um único operador linear $A_{L} \in L\left(X_{1} \otimes \cdots \otimes X_{n} ; Y\right)$, chamado de linearização de $A$, tal que $A=A_{L} \circ \sigma_{n}$, ou seja, tal que o diagrama

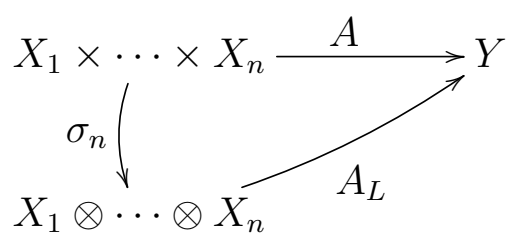

é comutativo. Mais ainda, a correspondência $A \longleftrightarrow A_{L}$ é um isomorfismo entre os espaços vetoriais $L\left(X_{1}, \ldots, X_{n}, Y\right)$ e $L\left(X_{1} \otimes \cdots \otimes X_{n}, Y\right)$.

Proposição 1.2.12. [71, Proposição 3.3] Sejam $E_{1}, \ldots, E_{n}$ espaços vetoriais normados. Então a função

$$
\pi: E_{1} \otimes \cdots \otimes E_{n} \longrightarrow[0, \infty), \pi(x)=\pi\left(\sum_{j=1}^{k} x_{j}^{1} \otimes \cdots \otimes x_{j}^{n}\right)=\inf \left\{\sum_{j=1}^{k}\left\|x_{j}^{1}\right\| \cdots\left\|x_{j}^{n}\right\|\right\}
$$

onde o infimo é tomado sobre todas as representações de $x$, é uma norma em $E_{1} \otimes \cdots \otimes E_{n}$. Além disso,

$$
\pi\left(x_{1} \otimes \cdots \otimes x_{n}\right)=\left\|x_{1}\right\| \cdots\left\|x_{n}\right\|
$$

para quaisquer $x_{i} \in E_{i}, i=1, \ldots, n$.

A norma $\pi$ é chamada de norma projetiva e o espaço normado $\left(E_{1} \otimes \cdots \otimes E_{n}, \pi\right)$ é denotado por $E_{1} \otimes_{\pi} \cdots \otimes_{\pi} E_{n}$. Se pelo menos dois dos espaços $E_{1}, \ldots, E_{n}$ têm dimensão infinita, então $E_{1} \otimes_{\pi} \cdots \otimes_{\pi} E_{n}$ é um espaço normado incompleto (veja [68, Teorema 3.24]). Por isso precisamos passar para o seu completamento, denotado por $E_{1} \widehat{\otimes}_{\pi} \cdots \widehat{\otimes}_{\pi} E_{n}$ e chamado de produto tensorial projetivo.

A seguir, temos a propriedade universal do produto tensorial projetivo. 
Teorema 1.2.13. [67, Theorem 2.9] Sejam $E_{1}, \ldots, E_{n}$ e $F$ espaços de Banach. Para todo operador $n$-linear $A \in \mathcal{L}\left(E_{1}, \ldots, E_{n} ; F\right)$ existe um único operador linear contínuo $A_{L} \in \mathcal{L}\left(E_{1} \widehat{\otimes}_{\pi} \cdots \widehat{\otimes}_{\pi} E_{n} ; F\right)$ tal que o diagrama

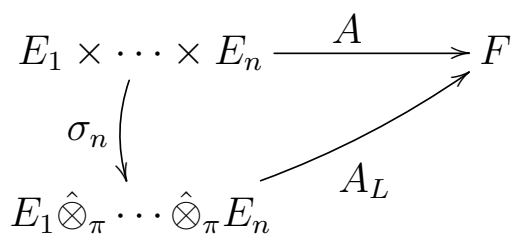

seja comutativo, ou seja, $A=A_{L} \circ \sigma_{n}$. Além disso, a correspondência $A \longleftrightarrow A_{L}$ é um isomorfismo isométrico entre os espaços de Banach $\mathcal{L}\left(E_{1}, \ldots, E_{n} ; F\right)$ e $\mathcal{L}\left(E_{1} \widehat{\otimes}_{\pi} \cdots \widehat{\otimes}_{\pi} E_{n} ; F\right)$.

No caso particular em que $F=\mathbb{K}$ tem-se o isomorfismo isométrico

$$
\ell: \mathcal{L}\left(E_{1}, \ldots, E_{n}\right) \longrightarrow\left(E_{1} \widehat{\otimes}_{\pi} \cdots \widehat{\otimes}_{\pi} E_{n}\right)^{*}, \ell(A)=A_{L} .
$$

Observação 1.2.14. Tomando $n=2$ e $F=\mathbb{K}$ no teorema anterior obtemos a identificação entre os espaços $\mathcal{L}\left(E_{1}, E_{2} ; \mathbb{K}\right)$ e $\left(E_{1} \hat{\otimes}_{\pi} E_{2}\right)^{*}$. Em particular, existe um operador

$$
U:\left(E_{1}^{*} \hat{\otimes}_{\pi} E_{2}\right)^{*} \longrightarrow \mathcal{L}\left(E_{1}^{*}, E_{2} ; \mathbb{K}\right)
$$

que é um isomorfismo isométrico. Para mais detalhes veja [33, p. 27] ou [66, Theorem 2.9].

O resultado a seguir é bem conhecido e muito fácil de ser demonstrado.

Lema 1.2.15. Se $T \in \mathcal{L}\left(E_{1}, \ldots, E_{n}\right)$ e $y \in F$, então $(T \otimes y)_{L}=T_{L} \otimes y$.

Definição 1.2.16. Dado $n \in \mathbb{N}$, denotamos por $S_{n}$ o conjunto de todas as bijeções do conjunto $\{1, \ldots, n\}$ sobre si mesmo. Dizemos que um operador $n$-linear $A \in L\left({ }^{n} E ; F\right)$ é simétrico se

$$
A\left(x_{1}, \ldots, x_{n}\right)=A\left(x_{\sigma(1)}, \ldots, x_{\sigma(n)}\right),
$$

para quaisquer $x_{1}, \ldots, x_{n} \in E$ e toda bijeção $\sigma \in S_{n}$. O subespaço vetorial de $L\left({ }^{n} E ; F\right)$ dos operadores $n$-lineares simétricos de $E$ em $F$ é denotado por $L^{s}\left({ }^{n} E ; F\right)$.

\subsection{Ideais de operadores lineares}

O conceito de ideal de operadores lineares foi introduzido por A. Pietsch em [59], obra na qual uma grande quantidade de exemplos pode ser encontrada. Informações adicionais e mais exemplos podem ser encontrados no livro de [33] de Defant e Floret. 
Definição 1.3.1. Um ideal de operadores $\mathcal{I}$ é uma subclasse da classe dos operadores lineares contínuos entre espaços de Banach tal que, para todos espaços de Banach $E$ e $F$, a componente

$$
\mathcal{I}(E ; F):=\mathcal{L}(E ; F) \cap \mathcal{I}
$$

satisfaz as seguintes condições:

(1) $\mathcal{I}(E ; F)$ é um subespaço vetorial de $\mathcal{L}(E ; F)$ que contém os operadores lineares de posto finito.

(2) A propriedade de ideal: se $u_{1} \in \mathcal{L}(G ; E), u_{2} \in \mathcal{I}(E ; F)$ e $u_{3} \in \mathcal{L}(F ; H)$, então $u_{3} \circ u_{2} \circ u_{1} \in \mathcal{I}(G ; H)$.

Se existe uma função $\|\cdot\|_{\mathcal{I}}: \mathcal{I} \longrightarrow[0, \infty)$ tais que:

(a) A função $\|\cdot\|_{\mathcal{I}}$ restrita à componente $\mathcal{I}(E ; F)$ é uma norma para quaisquer espaços de Banach $E$ e $F$;

(b) O funcional $I d_{\mathbb{K}}: \mathbb{K} \longrightarrow \mathbb{K}$ dado por $I d_{\mathbb{K}}(\lambda)=\lambda$ é tal que $\left\|I d_{\mathbb{K}}\right\|_{\mathcal{I}}=1$;

(c) Se $u_{1} \in \mathcal{L}(G ; E), u_{2} \in \mathcal{I}(E ; F)$ e $u_{3} \in \mathcal{L}(F ; H)$, então

$$
\left\|u_{3} \circ u_{2} \circ u_{1}\right\|_{\mathcal{I}} \leq\left\|u_{3}\right\| \cdot\left\|u_{2}\right\|_{\mathcal{I}} \cdot\left\|u_{1}\right\|
$$

então $\left(\mathcal{I},\|\cdot\|_{\mathcal{I}}\right)$ é um ideal normado de operadores lineares.

Além disso, se todas as componentes de $\mathcal{I}(E ; F)$ forem espaços completos relativamente à topologia gerada pela norma $\|\cdot\|_{\mathcal{I}}$, então dizemos que $\left(\mathcal{I},\|\cdot\|_{\mathcal{I}}\right)$ é um ideal de Banach.

Definição 1.3.2. Seja $\mathcal{I}$ um ideal de operadores. Definimos o fecho $\overline{\mathcal{I}}$ de $\mathcal{I}$ por

$$
\overline{\mathcal{I}}(E ; F):=\overline{\mathcal{I}(E ; F)} \|^{\|\cdot\|}
$$

para todos espaços de Banach $E$ e $F$. É sabido que $\overline{\mathcal{I}}$ também é um ideal de operadores.

Em particular, o fecho $\overline{\mathcal{F}}$ do ideal $\mathcal{F}$ dos operadores de posto finito é um ideal de operadores, e tais operadores são chamados de operadores aproximáveis.

Ao longo do texto trabalharemos com vários outros ideais de operadores, por exemplo os nucleares e suas variações. Optamos por defini-los na seção seguinte apenas para evitar repetições de conceitos, e assim garantir melhor fluidez no texto.

A título de exemplo, definimos a seguir o ideal dos operadores nucleares, que será muito utilizado nos próximos capítulos. 
Definição 1.3.3. [59] Um operador $u \in \mathcal{L}(E ; F)$ é chamado nuclear se existem sequências $\left(\varphi_{j}\right)_{j=1}^{\infty} \in E^{*}$ e $\left(y_{j}\right)_{j=1}^{\infty} \in F$ tais que

$$
u=\sum_{j=1}^{\infty} \varphi_{j} \otimes y_{j}
$$

Neste caso dizemos que $\sum_{j=1}^{\infty} \varphi_{j} \otimes y_{j}$ é uma representação nuclear de $u$ e definimos

$$
\|u\|_{\mathcal{N}}:=\inf \left\{\sum_{j=1}^{\infty}\left\|\varphi_{j}\right\| \cdot\left\|y_{j}\right\|\right\}
$$

onde o ínfimo é tomado sobre todas as chamadas representações nucleares de $u$. Denotaremos a classe de todos os operadores lineares nucleares de $E$ em $F$ por $\mathcal{N}(E ; F)$.

A classe $\left(\mathcal{N},\|\cdot\|_{\mathcal{N}}\right)$ dos operadores lineares nucleares é um ideal de Banach. Na presença da propriedade da aproximação, os espaços dos operadores nucleares têm a seguinte descrição, que é muito útil.

Proposição 1.3.4. [33, Corollary 1, p. 65] Sejam E e F espaços de Banach. Se E* ou F tem a propriedade da aproximação, então existe um isomorfismo isométrico $T: E^{*} \widehat{\otimes}_{\pi} F \longrightarrow$ $\mathcal{N}(E ; F)$ tal que

$$
T(\varphi \otimes y)(x)=\varphi(x) y
$$

para todos $\varphi \in E^{*}$ e $y \in F$.

\subsection{Multi-ideais de operadores multilineares}

Em [60], Pietsch generalizou o conceito de ideal para o caso dos operadores multilineares. Nos referimos também a [43, 44].

Definição 1.4.1. Um ideal de operadores multilineares, ou multi-ideal, é uma subclasse $\mathcal{M}$ da classe dos operadores multilineares contínuos entre espaços de Banach tal que, para todo $n \in \mathbb{N}$ e todos espaços de Banach $E_{1}, \ldots, E_{n}$ e $F$, a componente

$$
\mathcal{M}\left(E_{1}, \ldots, E_{n} ; F\right):=\mathcal{L}\left(E_{1}, \ldots, E_{n} ; F\right) \cap \mathcal{M}
$$

satisfaz as seguintes condições: 
(1) $\mathcal{M}\left(E_{1}, \ldots, E_{n} ; F\right)$ é subespaço vetorial de $\mathcal{L}\left(E_{1}, \ldots, E_{n} ; F\right)$ que contém os operadores $n$-lineares de tipo finito.

(2) A propriedade de multi-ideal: se $A \in \mathcal{M}\left(E_{1}, \ldots, E_{n} ; F\right), u_{j} \in \mathcal{L}\left(G_{j} ; E_{j}\right), j=$ $1, \ldots, n$ e $t \in \mathcal{L}(F ; H)$, então a composição to $A \circ\left(u_{1}, \ldots, u_{n}\right)$ pertence a $\mathcal{M}\left(G_{1}, \ldots, G_{n} ; H\right)$.

Se existe uma função $\|\cdot\|_{\mathcal{M}}: \mathcal{M} \longrightarrow[0, \infty)$ tais que:

(a) A função $\|\cdot\|_{\mathcal{M}}$ restrita à componente $\mathcal{M}\left(E_{1}, \ldots, E_{n} ; F\right)$ é uma norma para todo $n \in \mathbb{N}$ e quaisquer espaços de Banach $E_{1}, \ldots, E_{n}$ e $F$;

(b) A aplicação $n$-linear $I_{n}: \mathbb{K}^{n} \longrightarrow \mathbb{K}$, dada por $I_{n}\left(\lambda_{1}, \ldots, \lambda_{n}\right)=\lambda_{1} \cdots \lambda_{n}$, é tal que $\left\|I_{n}\right\|_{\mathcal{M}}=1$

(c) Se $A \in \mathcal{M}\left(E_{1}, \ldots, E_{n} ; F\right), u_{j} \in \mathcal{L}\left(G_{j} ; E_{j}\right), j=1, \ldots, n$ e $t \in \mathcal{L}(F ; G)$, então a composta satisfaz

$$
\left\|t \circ A \circ\left(u_{1}, \ldots, u_{n}\right)\right\|_{\mathcal{M}} \leq\|t\| \cdot\|A\|_{\mathcal{M}} \cdot\left\|u_{1}\right\| \cdots\left\|u_{n}\right\|
$$

então $\left(\mathcal{M},\|\cdot\|_{\mathcal{M}}\right)$ é chamado de multi-ideal normado.

Mais ainda, se todas as componentes $\mathcal{M}\left(E_{1}, \ldots, E_{n} ; F\right)$ são subespaços completos relativamente à topologia gerada pela norma $\|\cdot\|_{\mathcal{M}}$, então dizemos que $\left(\mathcal{M},\|\cdot\|_{\mathcal{M}}\right)$ é um multi-ideal de Banach.

Veremos a seguir alguns exemplos de multi-ideais de Banach que serão utilizados no desenvolvimento da tese.

Exemplo 1.4.2. [60] A classe dos operadores multilineares de tipo finito $\mathcal{L}_{f}$ e seu fecho $\overline{\mathcal{L}_{f}}$, isto é, os operadores que podem ser aproximados, na norma usual, por operadores de tipo finito, são multi-ideais.

Exemplo 1.4.3. [35, Definition 1.26] Um operador $n$-linear $A \in \mathcal{L}\left(E_{1}, \ldots, E_{n} ; F\right)$ é $n u$ clear se, para $i=1, \ldots, n$, existem uma sequência limitada $\left(\varphi_{j}^{(i)}\right)_{j=1}^{\infty} \subseteq E_{i}^{*}$, uma sequência $\left(\lambda_{j}\right)_{j=1}^{\infty} \in \ell_{1}$ e uma sequência limitada $\left(y_{j}\right)_{j=1}^{\infty}$ em $F$, tais que

$$
A\left(x_{1}, \ldots, x_{n}\right)=\sum_{j=1}^{\infty} \lambda_{j} \varphi_{j}^{(1)} \otimes \cdots \otimes \varphi_{j}^{(n)} \otimes y_{j}\left(x_{1}, \ldots, x_{n}\right)=\sum_{j=1}^{\infty} \lambda_{j} \varphi_{j}^{(1)}\left(x_{1}\right) \cdots \varphi_{j}^{(n)}\left(x_{n}\right) y_{j}
$$


para todo $\left(x_{1}, \ldots, x_{n}\right) \in E_{1} \times \cdots \times E_{n}$. Neste caso, dizemos que $\sum_{j=1}^{\infty} \lambda_{j} \varphi_{j}^{(1)} \otimes \cdots \otimes \varphi_{j}^{(n)} \otimes y_{j}$ é uma representação nuclear de $A$ e escrevemos $A \in \mathcal{L}_{\mathcal{N}}\left(E_{1}, \ldots, E_{n} ; F\right)$. Definimos ainda

$$
\|A\|_{\mathcal{L}_{\mathcal{N}}}:=\inf \left\{\sum_{j=1}^{\infty}\left|\lambda_{j}\right| \cdot\left\|\varphi_{j}^{(1)}\right\| \cdots\left\|\varphi_{j}^{(n)}\right\| \cdot\left\|y_{j}\right\|\right\}
$$

onde o ínfimo é tomado sobre todas as representações nucleares de $A$.

Exemplo 1.4.4. [15, Definition 2.2] Seja $1 \leq p<\infty$. Um operador $n$-linear $A \in$ $\mathcal{L}\left(E_{1}, \ldots, E_{n} ; F\right)$ é $\sigma(p)$-nuclear se existem sequências $\left(\lambda_{j}\right)_{j=1}^{\infty} \in \ell_{p^{*}},\left(\varphi_{i j}\right)_{j=1}^{\infty}$ em $E_{i}^{*}$, para $i=1, \ldots, n$, e $\left(y_{j}\right)_{j=1}^{\infty}$ em $F$ tais que

$$
\begin{gathered}
A=\sum_{j=1}^{\infty} \lambda_{j} \varphi_{1 j} \otimes \cdots \otimes \varphi_{n j} \otimes y_{j}, \sup _{x_{i} \in B_{E_{i}}, y^{*} \in B_{F^{*}}}\left(\sum_{j=1}^{\infty}\left|\varphi_{1 j}\left(x_{1}\right) \cdots \varphi_{n j}\left(x_{n}\right) y^{*}\left(y_{j}\right)\right|^{p}\right)^{\frac{1}{p}}<\infty \mathrm{e} \\
\lim _{m \rightarrow \infty} \sup _{x_{i} \in B_{E_{i}}, y^{*} \in B_{F^{*}}}\left(\sum_{j=m}^{\infty}\left|\varphi_{1 j}\left(x_{1}\right) \cdots \varphi_{j n}\left(x_{n}\right) y^{*}\left(y_{j}\right)\right|^{p}\right)^{\frac{1}{p}}=0
\end{gathered}
$$

Neste caso dizemos que $\sum_{j=1}^{\infty} \lambda_{j} \varphi_{1 j} \otimes \cdots \varphi_{n j} \otimes y_{j}$ é uma representação $\sigma(p)$-nuclear de A. Definimos ainda

$$
\|A\|_{\sigma(p)}:=\inf \left\{\left\|\left(\lambda_{j}\right)_{j=1}^{\infty}\right\|_{p^{*}} \cdot \sup _{x_{i} \in B_{E_{i}}, y^{*} \in B_{F^{*}}}\left(\sum_{j=1}^{\infty}\left|\varphi_{1 j}\left(x_{1}\right) \cdots \varphi_{j n}\left(x_{n}\right) y^{*}\left(y_{j}\right)\right|^{p}\right)^{\frac{1}{p}}\right\},
$$

onde o ínfimo é tomado sobre todas as representações $\sigma(p)$-nucleares de $A$. Denotaremos o subespaço dos operadores $n$-lineares $\sigma(p)$-nucleares por $\mathcal{L}_{\sigma(p)}\left(E_{1}, \ldots, E_{n} ; F\right)$.

No caso linear $n=1$, o ideal dos operadores lineares $\sigma(p)$-nucleares será denotado por $\mathcal{N}_{\sigma(p)}$.

Exemplo 1.4.5. [52] Sejam $s, r_{k} \in[0, \infty], k=1, \ldots, n$ tais que $\frac{1}{s} \leq \frac{1}{r_{1}}+\cdots+\frac{1}{r_{n}}$. Um operador $n$-linear $A \in \mathcal{L}\left(E_{1}, \ldots, E_{n} ; F\right)$ é chamado de absolutamente $\left(s ; r_{1}, \ldots, r_{n}\right)$ somante se existe $C \geq 0$ tal que

$$
\left\|\left(A\left(x_{1 j}, \ldots, x_{n j}\right)\right)_{j=1}^{m}\right\|_{s} \leq C \cdot \prod_{k=1}^{n}\left\|\left(x_{k j}\right)_{j=1}^{m}\right\|_{w, r_{k}},
$$

para $m \in \mathbb{N}, x_{k j} \in E_{k}, k=1, \ldots, n$ e $j=1, \ldots, m$. 
Denotaremos o subespaço dos operadores multilineares absolutamente $\left(s ; r_{1}, \ldots, r_{n}\right)$ somante por $\mathcal{L}_{a s}^{\left(s ; r_{1}, \ldots, r_{n}\right)}\left(E_{1}, \ldots, E_{n} ; F\right)$ e o ínfimo das constantes que satisfazem a desigualdade (1.3) por $\|\cdot\|_{a s\left(s ; r_{1}, \ldots, r_{n}\right)}$.

Quando $n=1$ e, portanto $r_{1}=\cdots=r_{n}=r$, denotamos o ideal de Banach dos operadores lineares absolutamente $(s ; r)$-somantes por $\Pi_{s, r}$, no lugar de $\mathcal{L}_{a s}^{s, r}$ (veja [34]).

Exemplo 1.4.6. [52, Definition 2.1] Sejam $s \in[1, \infty], r_{k} \in[1, \infty], k=1, \ldots, n$ tais que

$$
1 \leq \frac{1}{s}+\frac{1}{r_{1}^{*}}+\cdots+\frac{1}{r_{n}^{*}}
$$

Um operador $n$-linear $A \in \mathcal{L}\left(E_{1}, \ldots, E_{n} ; F\right)$ é dito ser $\left(s ; r_{1}, \ldots, r_{n}\right)$-nuclear se existem sequências $\left(\lambda_{j}\right)_{j=1}^{\infty} \in \ell_{s},\left(y_{j}\right)_{j=1}^{\infty}$ em $\ell_{\infty}(F)$ e $\left(\varphi_{k j}\right)_{j=1}^{\infty}$ em $\ell_{r_{k}^{*}}^{w}\left(E_{k}^{*}\right), k=1, \ldots, n$, tais que

$$
A\left(x_{1}, \ldots, x_{n}\right)=\sum_{j=1}^{\infty} \lambda_{j}\left(\varphi_{1 j} \otimes \cdots \otimes \varphi_{n j} \otimes y_{j}\right)\left(x_{1}, \ldots, x_{n}\right),
$$

para todo $\left(x_{1}, \ldots, x_{n}\right) \in E_{1} \times \cdots \times E_{n}$. Neste caso dizemos que $\sum_{j=1}^{\infty} \lambda_{j} \varphi_{1 j} \otimes \cdots \otimes \varphi_{n j} \otimes y_{j}$ é uma representação $\left(s ; r_{1}, \ldots, r_{n}\right)$-nuclear de $A$.

Denotamos o espaço vetorial desses operadores por $\mathcal{L}_{\mathcal{N}}^{\left(s ; r_{1}, \ldots, r_{n}\right)}\left(E_{1}, \ldots, E_{n} ; F\right)$ e definimos

$$
\|A\|_{\mathcal{N}_{\left(s ; r_{1}, \ldots, r_{n}\right)}}=\inf \left\{\left\|\left(\lambda_{j}\right)_{j=1}^{\infty}\right\|_{s} \cdot\left\|\left(y_{j}\right)_{j=1}^{\infty}\right\|_{\infty} \cdot\left\|\left(\varphi_{1 j}\right)_{j=1}^{\infty}\right\|_{w, r_{1}^{*}} \cdots\left\|\left(\varphi_{n j}\right)_{j=1}^{\infty}\right\|_{w, r_{n}^{*}}\right\}
$$

onde o ínfimo é tomado sobre todas as representações $\left(s ; r_{1}, \ldots, r_{n}\right)$-nucleares de $A$.

No caso linear $n=1$, com $1 \leq \frac{1}{s}+\frac{1}{r}$, denotaremos por $\left(\mathcal{N}_{(s ; r)},\|\cdot\|_{\mathcal{N}_{(s ; r)}}\right)$ o ideal de Banach dos operadores lineares $(s ; r)$-nucleares. Para $s=1$ e $r=\infty$, temos o ideal dos operadores $(1, \infty)$-nucleares que será denotado simplesmente por $\mathcal{N}$. Note que recaímos no ideal visto na Definição 1.3.3. Para mais detalhes sugerimos [33, 59].

Estudaremos agora os multi-ideais de composição, que serão fundamentais nos principais resultados dos próximos três capítulos.

Definição 1.4.7. [17, Definitions 3.1, 3.6] Seja $\left(\mathcal{I},\|\cdot\|_{\mathcal{I}}\right)$ um ideal normado de operadores. Dizemos que um operador $A \in \mathcal{L}\left(E_{1}, \ldots, E_{n} ; F\right)$ pertence a $\mathcal{I} \circ \mathcal{L}\left(E_{1}, \ldots, E_{n} ; F\right)$ se existem um espaço de Banach $G$, um operador linear $u \in \mathcal{I}(G ; F)$ e uma aplicação $n$-linear $B \in \mathcal{L}\left(E_{1}, \ldots, E_{n} ; G\right)$ tais que

$$
A=u \circ B .
$$




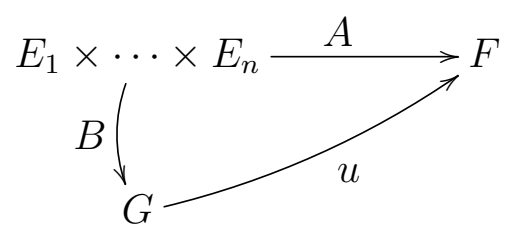

Neste caso definimos

$$
\|A\|_{\mathcal{I} \circ \mathcal{L}}:=\inf \left\{\|u\|_{\mathcal{I}} \cdot\|B\|: A=u \circ B, B \in \mathcal{L}\left(E_{1}, \ldots, E_{n} ; G\right), u \in \mathcal{I}(G ; F)\right\}
$$

Proposição 1.4.8. [17, Proposition 3.2] Seja $\mathcal{I}$ um ideal de operadores. As seguintes condições são equivalentes para um operador $A \in \mathcal{L}\left(E_{1}, \ldots, E_{n} ; F\right)$ :

(a) $A \in \mathcal{I} \circ \mathcal{L}\left(E_{1}, \ldots, E_{n} ; F\right)$.

(b) $A_{L} \in \mathcal{I}\left(E_{1} \widehat{\otimes}_{\pi} \cdots \widehat{\otimes}_{\pi} E_{n} ; F\right)$.

Proposição 1.4.9. [17, Proposition 3.7] Seja $\left(\mathcal{I},\|\cdot\|_{\mathcal{I}}\right)$ um ideal de Banach de operadores. Então $\|\cdot\|_{\mathcal{I} \circ \mathcal{L}}$ torna $\mathcal{I} \circ \mathcal{L}$ um multi-ideal de Banach. Além disso, $\|A\|_{\mathcal{I} \circ \mathcal{L}}=\left\|A_{L}\right\|_{\mathcal{I}}$ para todo $A \in \mathcal{I} \circ \mathcal{L}\left(E_{1}, \ldots, E_{n} ; F\right)$.

Vejamos que alguns multi-ideais clássicos são ideais de composição. Dizemos que um operador $A \in \mathcal{L}\left(E_{1}, \ldots, E_{n} ; F\right)$ é compacto (fracamente compacto, respectivamente) se $A\left(B_{E_{1}} \times \cdots \times B_{E_{n}}\right)$ é um subconjunto relativamente compacto (relativamente fracamente compacto, respectivamente) de $F$. Por $\mathcal{L}_{\mathcal{K}}$ e $\mathcal{L}_{\mathcal{W}}$ denotamos as classes dos operadores multilineares compactos e relativamente compactos entre espaços de Banach, e por $\mathcal{W}$ o ideal dos operadores lineares fracamente compactos.

Proposição 1.4.10. Sejam $E_{1}, \ldots, E_{n}$ e $F$ espaços de Banach.

(a) $\mathcal{L}_{\mathcal{K}}\left(E_{1}, \ldots, E_{n} ; F\right)=\mathcal{K} \circ \mathcal{L}\left(E_{1}, \ldots, E_{n} ; F\right)$ e $\mathcal{L}_{\mathcal{W}}\left(E_{1}, \ldots, E_{n} ; F\right)=\mathcal{W} \circ \mathcal{L}\left(E_{1}, \ldots, E_{n} ; F\right)$.

(b) $\overline{\mathcal{L}_{\mathcal{F}}}\left(E_{1}, \ldots, E_{n} ; F\right)=\overline{\mathcal{F}} \circ \mathcal{L}\left(E_{1}, \ldots, E_{n} ; F\right)$ isometricamente.

Para o item (a) veja [66, Lemma 4.1], e para o item (b) veja [19, Theorem 2.2].

\subsection{Hiper-ideais de operadores multilineares}

A teoria de multi-ideais, que consideram a composição de um operador multilinear com operadores lineares dos dois lados, foi refinada no sentido de considerar classes de operadores multilineares que são estáveis pela composição com operadores multilineares à esquerda e operadores lineares à direita. 
Definição 1.5.1. [20, 22, 74] Um hiper-ideal de operadores multilineares é uma subclasse $\mathcal{H}$, da classe dos operadores multilineares contínuos entre espaços de Banach tal que, para todo $n \in \mathbb{N}$ e todos espaços de Banach $E_{1}, \ldots, E_{n}$ e $F$, a componente

$$
\mathcal{H}\left(E_{1}, \ldots, E_{n} ; F\right):=\mathcal{L}\left(E_{1}, \ldots, E_{n} ; F\right) \cap \mathcal{H}
$$

satisfaz as seguintes condições:

(1) $\mathcal{H}\left(E_{1}, \ldots, E_{n} ; F\right)$ é um subespaço vetorial de $\mathcal{L}\left(E_{1}, \ldots, E_{n} ; F\right)$ que contém os operadores multilineares de tipo finito.

(2) Propriedade de hiper-ideal: Dados números naturais $n$ e $1 \leq m_{1}<\cdots<m_{n}$, espaços de Banach $G_{1}, \ldots, G_{m_{n}}, E_{1}, \ldots, E_{n}, F$ e $H$, se

$$
B_{1} \in \mathcal{L}\left(G_{1}, \ldots, G_{m_{1}} ; E_{1}\right), \ldots, B_{n} \in \mathcal{L}\left(G_{m_{n-1}+1}, \ldots, G_{m_{n}} ; E_{n}\right)
$$

$t \in \mathcal{L}(F ; H)$ e $A \in \mathcal{H}\left(E_{1}, \ldots, E_{n} ; F\right)$, então $t \circ A \circ\left(B_{1}, \ldots, B_{n}\right) \in \mathcal{H}\left(G_{1}, \ldots, G_{m_{n}} ; H\right)$.

Se existe uma função $\|\cdot\|_{\mathcal{H}}: \mathcal{H} \rightarrow[0, \infty)$ tal que:

(a) A função $\|\cdot\|_{\mathcal{H}}$ restrita à componente $\mathcal{H}\left(E_{1}, \ldots, E_{n} ; F\right)$ é uma norma para quaisquer espaços de Banach $E_{1}, \ldots, E_{n}, F$ e todo $n \in \mathbb{N}$;

(b) O operador $n$-linear $I_{n}: \mathbb{K}^{n} \rightarrow \mathbb{K}$, dado por $I_{n}\left(\lambda_{1}, \ldots, \lambda_{n}\right)=\lambda_{1} \cdots \lambda_{n}$, é tal que $\left\|I_{n}\right\|_{\mathcal{H}}=1$

(c) Se $A \in \mathcal{H}\left(E_{1}, \ldots, E_{n} ; F\right), t \in \mathcal{L}(F ; H)$ e

$$
B_{1} \in \mathcal{L}\left(G_{1}, \ldots, G_{m_{1}} ; E_{1}\right), \ldots, B_{n} \in \mathcal{L}\left(G_{m_{n-1}+1}, \ldots, G_{m_{n}} ; E_{n}\right),
$$

então

$$
\left\|t \circ A \circ\left(B_{1}, \ldots, B_{n}\right)\right\|_{\mathcal{H}} \leq\|t\| \cdot\|A\|_{\mathcal{H}} \cdot\left\|B_{1}\right\| \cdots\left\|B_{n}\right\|
$$

então $\left(\mathcal{H} ;\|\cdot\|_{\mathcal{H}}\right)$ é chamado de hiper-ideal normado. Mais ainda, se todas as componentes $\mathcal{H}\left(E_{1}, \ldots, E_{n} ; F\right)$ são espaços completos relativamente à norma $\|\cdot\|_{\mathcal{H}}$, então dizemos que $\left(\mathcal{H} ;\|\cdot\|_{\mathcal{H}}\right)$ é um hiper-ideal de Banach.

Dizemos que um ideal de operadores (ou um multi-ideal ou um hiper-ideal) é fechado se todas as suas componentes são fechadas em relação à norma usual.

Exemplos importantes de hiper-ideais são os ideais de composição: 
Teorema 1.5.2. [72, Teorema 2.4.1] Se $\mathcal{I}$ é um ideal de operadores, então $\mathcal{I} \circ \mathcal{L}$ é um hiper-ideal. Se $\left(\mathcal{I},\|\cdot\|_{\mathcal{I}}\right)$ é ideal de operadores normado (de Banach, respectivamente), então $\left(\mathcal{I} \circ \mathcal{L},\|\cdot\|_{\mathcal{I} \circ \mathcal{L}}\right)$ é hiper-ideal normado (de Banach, respectivamente). Em particular, se $\mathcal{I}$ é fechado, então $\mathcal{I} \circ \mathcal{L}$ é um hiper-ideal fechado.

Em particular, as classes dos operadores multilineares compactos $\mathcal{L}_{\mathcal{K}}$, fracamente compactos $\mathcal{L}_{\mathcal{W}}$ e aproximáveis $\overline{\mathcal{L}_{\mathcal{F}}}$ são hiper-ideais fechados (basta combinar o Teorema 1.5.2 com a Proposição 1.4.10).

\subsection{Polinômios homogêneos}

Também para a teoria geral de polinômios homogêneos referimos os livros de Mujica [57] e Dineen [36].

Dados um operador $A: E^{n} \longrightarrow F$ e $x \in E$, escreveremos $A\left(x^{n}\right)$ no lugar de $A(x, \stackrel{(n)}{.}, x)$.

Definição 1.6.1. Sejam $E$ e $F$ espaços normados. Uma aplicação $P: E \longrightarrow F$ é chamada de polinômio n-homogêneo se existe um operador $n$-linear $A \in L\left({ }^{n} E ; F\right)$ tal que $P(x)=$ $A\left(x^{n}\right)$ para todo $x \in E$. Neste caso dizemos que o polinômio $n$-homogêneo $P$ é gerado pelo operador $n$-linear $A$ escrevemos $P=\hat{A}$.

Denotamos os espaços dos polinômios $n$-homogêneos de $E$ em $F$ e dos polinômios $n$-homogêneos contínuos de $E$ em $F$ por $P\left({ }^{n} E ; F\right)$ e $\mathcal{P}\left({ }^{n} E ; F\right)$, respectivamente. No caso em que $F=\mathbb{K}$, escrevemos $P\left({ }^{n} E\right)$ e $\mathcal{P}\left({ }^{n} E\right)$.

A expressão

$$
P \in \mathcal{P}\left({ }^{n} E ; F\right) \mapsto\|P\|:=\sup \{\|P(x)\|: x \in E \text { e }\|x\| \leq 1\}
$$

define uma norma em $\mathcal{P}\left({ }^{n} E ; F\right)$. Se $F$ é espaço de Banach, então $\left(\mathcal{P}\left({ }^{n} E ; F\right),\|\cdot\|\right)$ também é um espaço de Banach (veja [57, Corollary 2.3]).

Mantendo a notação, no que segue $E, E_{1}, \ldots, E_{n}, F, G$ e $H$ denotarão espaços de Banach.

Proposição 1.6.2. [57, Theorem 2.2] A correspondência $A \mapsto \hat{A}$ que associa a cada operador n-linear simétrico $A \in L^{s}\left({ }^{n} E ; F\right)$ seu polinômio associado $\hat{A} \in P\left({ }^{n} E ; F\right)$ induz um isomorfismo de espaços vetoriais entre $L^{s}\left({ }^{n} E ; F\right)$ e $P\left({ }^{n} E ; F\right)$. E mais, esse isomorfismo induz um isomorfismo topológico entre $\mathcal{L}^{s}\left({ }^{n} E ; F\right)$ e $\mathcal{P}\left({ }^{n} E ; F\right)$ e

$$
\|\hat{A}\| \leq\|A\| \leq \frac{n^{n}}{n !}\|\hat{A}\|
$$


para toda $A \in \mathcal{L}^{s}\left({ }^{n} E ; F\right)$.

Dado um polinômio $P \in \mathcal{P}\left({ }^{n} E ; F\right)$, denotaremos por $\check{P} \in \mathcal{L}^{s}\left({ }^{n} E ; F\right)$ o único operador $n$-linear simétrico associado a $P$.

Proposição 1.6.3. [57, Proposition 2.4] As seguintes afirmações são equivalentes para um polinômio n-homogêneo $P \in P\left({ }^{n} E ; F\right)$ :

(a) $P \in \mathcal{P}\left({ }^{n} E ; F\right)$.

(b) P é contínuo na origem.

(c) Existe um constante $C>0$ tal que $\|P(x)\| \leq C\|x\|^{n}$ para todo $x \in E$.

(d) $\sup \{\|P(x)\|: x \in E$ e $\|x\| \leq 1\}<\infty$.

(e) Existe $A \in \mathcal{L}\left({ }^{n} E ; F\right)$ tal que $P=\hat{A}$.

(f) O operador n-linear simétrico $\check{P}$ é contínuo.

Analogamente ao caso multilinear, definimos:

Definição 1.6.4. Um polinômio $P \in \mathcal{P}\left({ }^{n} E ; F\right)$ é de tipo finito se existem $k \in \mathbb{N}, \varphi_{j} \in E^{*}$ e $y_{j} \in F, \operatorname{com} j=1, \ldots, k$, tais que

$$
P(x)=\sum_{j=1}^{k} \varphi_{j}(x)^{n} y_{j}
$$

para todo $x \in E$.

Denotamos o subespaço dos polinômios $n$-homogêneos de tipo finito por $\mathcal{P}_{f}\left({ }^{n} E ; F\right)$.

Definição 1.6.5. Um polinômio $P \in \mathcal{P}\left({ }^{n} E ; F\right)$ é de posto finito se o subespaço de $F$ gerado pela imagem $P(E)$ de $P$ tem dimensão finita; ou equivalentemente, se existem $k \in \mathbb{N}, Q_{j} \in \mathcal{P}\left({ }^{n} E\right)$ e $y_{j} \in F, \operatorname{com} j=1, \ldots, k$, tais que

$$
P(x)=\sum_{j=1}^{k} Q_{j}(x) y_{j}
$$

para todo $x \in E$.

Denotamos o subespaço dos polinômios $n$-homogêneos de posto finito por $\mathcal{P}_{\mathcal{F}}\left({ }^{n} E ; F\right)$. 
Por simplicidade, dados $\varphi \in E^{*}, Q \in \mathcal{P}\left({ }^{n} E\right)$ e $y \in F$, denotamos os polinômios n-homogêneos

$$
x \in E \mapsto \varphi(x)^{n} y \quad \text { e } \quad x \in E \mapsto Q(x) y,
$$

por $\varphi^{n} \otimes y$ e $Q \otimes y$, respectivamente. Tem-se

$$
\left\|\varphi^{n} \otimes y\right\|=\|\varphi\|^{n} \cdot\|y\| \text { e }\|Q \otimes y\|=\|Q\| \cdot\|y\| .
$$

Para o produto tensorial projetivo de um espaço de Banach $E$ por ele mesmo, adotamos as seguintes notações simplificadas:

$$
\otimes^{n} E:=E \otimes(n) \cdot \otimes E \text { e } \otimes_{\pi}^{n} E:=E \otimes_{\pi} \stackrel{(n)}{\cdots} \otimes_{\pi} E .
$$

E para $x \in E$, escrevemos $\otimes^{n} x:=x \otimes \stackrel{(n)}{\cdots} \otimes x$.

Todas as informações do próximo parágrafo estão em Ryan [66] e Floret [42].

O subespaço vetorial de $\otimes^{n} E$ gerado pelos tensores da forma $\otimes^{n} x, x \in E$, é denotado por $\otimes^{n, s} E$. E o respectivo subespaço normado de $\otimes_{\pi}^{n} E$ é denotado por $\otimes_{\pi}^{n, s} E$. O completamento de $\otimes_{\pi}^{n, s} E$ com respeito à norma projetiva $\pi$ é denotado por $\widehat{\otimes}_{\pi}^{n, s} E$ e chamado de produto tensorial simétrico projetivo. Para fins topológicos, esse espaço é suficiente, mas para fins métricos precisamos considerar outra norma: para $z \in \otimes^{n, s} E$ define-se a norma s-projetiva de $z$ por

$$
\pi_{s}(z)=\inf \left\{\sum_{j=1}^{k}\left|\lambda_{j}\right| \cdot\left\|x_{j}\right\|^{n}: k \in \mathbb{N}, z=\sum_{j=1}^{k} \lambda_{j} \otimes^{n} x_{j}\right\} .
$$

O espaço normado resultante é denotado por $\otimes_{\pi_{s}}^{n, s} E$ e seu completamento $\widehat{\otimes}_{\pi_{s}}^{n, s} E$ é chamado de produto tensorial simétrico s-projetivo. As normas $\pi$ e $\pi_{s}$ são equivalentes mas não coincidentes. O operador

$$
x \in E \mapsto \rho_{n}(E):=\otimes^{n} x,
$$

é um polinômio $n$-homogêneo contínuo tanto tomando valores em $\widehat{\otimes}_{\pi}^{n, s} E$ como em $\widehat{\otimes}_{\pi_{s}}^{n, s} E$.

O próximo teorema exibe a propriedade universal do produto tensorial simétrico projetivo.

Teorema 1.6.6. [66, Proposition 2.1] Para todo polinômio n-homogêneo $P \in \mathcal{P}\left({ }^{n} E ; F\right)$ existe um único operador linear $P_{L} \in \mathcal{L}\left(\hat{\otimes}_{\pi}^{n, s} E ; F\right)$, chamado de linearização de $P$, tal que $P=P_{L} \circ \rho_{n}$, isto é,

$$
P(x)=P_{L}\left(\otimes^{n} x\right) \text { para todo } x \in E .
$$

Além disso, a correspondência $P \mapsto P_{L}$ é um isomorfismo topológico entre $\mathcal{P}\left({ }^{n} E ; F\right)$ e $\mathcal{L}\left(\hat{\otimes}_{\pi}^{n, s} E ; F\right)$. 
E a propriedade universal do produto tensorial simétrico $s$-projetivo funciona da seguinte forma:

Teorema 1.6.7. [42, Proposition 2.2(1)] Para todo polinômio n-homogêneo $P \in \mathcal{P}\left({ }^{n} E ; F\right)$ existe um único operador linear $P_{L, s} \in \mathcal{L}\left(\hat{\otimes}_{\pi_{s}}^{n, s} E ; F\right)$, também chamado de linearização de $P$, tal que $P=P_{L, s} \circ \rho_{n}$, isto é,

$$
P(x)=P_{L, s}\left(\otimes^{n} x\right) \text { para todo } x \in E .
$$

Além disso, o operador linearização

$$
\ell: \mathcal{P}\left({ }^{n} E ; F\right) \longrightarrow \mathcal{L}\left(\hat{\otimes}_{\pi_{s}}^{n, s} E ; F\right), \ell(P)=P_{L, s},
$$

é um isomorfismo isométrico.

O próximo teorema, devido a F. Blasco, será muito útil.

Teorema 1.6.8. [7, Theorem 3] O espaço $\widehat{\otimes}_{\pi}^{n, s} E$ é um subespaço complementado de $\widehat{\otimes}_{\pi}^{n+1, s}$ E para cada inteiro positivo $n \in \mathbb{N}$.

Aplicando o teorema acima repetidas vezes, existe um operador $i: E \longrightarrow \widehat{\otimes}_{\pi}^{n, s} E$ que é um isomorfismo sobre um subespaço complementado de $\widehat{\otimes}_{\pi}^{n, s} E$, o qual denominamos de operador de Blasco.

Definição 1.6.9. [6] Sejam $E, F$ espaços de Banach e $P \in \mathcal{P}\left({ }^{n} E ; F\right)$. O adjunto de Aron-Schottenloher de $P$ é definido por

$$
P^{*}: F^{*} \longrightarrow \mathcal{P}\left({ }^{n} E\right), P^{*}(\varphi)(y)=\varphi(P(y)),
$$

para todos $\varphi \in F^{*}$ e $y \in F$.

As propriedades básicas do adjunto de um polinômio são dadas no seguinte resultado.

Proposição 1.6.10. [61, Proposição 2.1.3] Sejam E, F espaços de Banach e $P \in \mathcal{P}\left({ }^{n} E ; F\right)$. Então, $P^{*} \in \mathcal{L}\left(F^{*} ; \mathcal{P}\left({ }^{n} E\right)\right)$ e $\|P\|=\left\|P^{*}\right\|$.

Necessitaremos de um outro resultado devido a Aron e Schottenloher:

Proposição 1.6.11. [6, Proposition 5.3] Sejam m e $n \in \mathbb{N}$ tais que $m \leq n$. Então $\mathcal{P}\left({ }^{m} E\right)$ é isomorfo a um subespaço complementado de $\mathcal{P}\left({ }^{n} E\right)$. 
Para a teoria de ideais de polinômios nos referimos a $[26,43,44]$.

Definição 1.6.12. Um ideal de polinômios homogêneos é uma subclasse $\mathcal{Q}$ da classe dos polinômios homogêneos contínuos entre espaços de Banach tal que, para todo $n \in \mathbb{N}$ e todos espaços de Banach $E$ e $F$, a componente

$$
\mathcal{Q}\left({ }^{n} E ; F\right):=\mathcal{P}\left({ }^{n} E ; F\right) \cap \mathcal{Q}
$$

satisfaz as seguintes condições:

(1) $\mathcal{Q}\left({ }^{n} E ; F\right)$ é um subespaço vetorial $\mathcal{P}\left({ }^{n} E ; F\right)$ que contém os polinômios de tipo finito.

(2) Propriedade de ideal: se $P \in \mathcal{Q}\left({ }^{n} E ; F\right), u \in \mathcal{L}(G ; E)$ e $t \in \mathcal{L}(F ; H)$, então $t \circ P \circ u \in \mathcal{Q}\left({ }^{n} G ; H\right)$.

Se existe uma função $\|\cdot\|_{\mathcal{Q}}: \mathcal{Q} \longrightarrow[0, \infty)$ tal que:

(a) A função $\|\cdot\|_{\mathcal{Q}}$ restrita à componente $\mathcal{Q}\left({ }^{n} E ; F\right)$ é uma norma para quaisquer espaços de Banach $E$ e $F$ e todo $n \in \mathbb{N}$;

(b) Se $\hat{I}_{n}: \mathbb{K} \longrightarrow \mathbb{K}$ é dado por $\hat{I}_{n}(\lambda)=\lambda^{n}$, então $\left\|\hat{I}_{n}\right\|_{\mathcal{Q}}=1$ para todo $n$;

(c) Se $u \in \mathcal{L}(G ; E), P \in \mathcal{Q}\left({ }^{n} E ; F\right)$ e $t \in \mathcal{L}(F ; H)$, então

$$
\|t \circ P \circ u\|_{\mathcal{Q}} \leq\|t\| \cdot\|P\|_{\mathcal{Q}} \cdot\|u\|^{n}
$$

então $\left(\mathcal{Q},\|\cdot\|_{\mathcal{Q}}\right)$ é chamado de ideal normado de polinômios. Além disso, se todas as componentes $\mathcal{Q}\left({ }^{n} E ; F\right)$ são espaços completos com respeito à norma $\|\cdot\|_{\mathcal{Q}}$, diremos que $\left(\mathcal{Q},\|\cdot\|_{\mathcal{Q}}\right)$ é um ideal de Banach de polinômios. E se cada componente $\mathcal{Q}\left({ }^{n} E ; F\right)$ é fechada na norma usual, dizemos que $\mathcal{Q}$ é um ideal fechado de polinômios.

Estudaremos agora os ideais de polinômios de composição.

Definição 1.6.13. [17, Definition 3.1] Seja $\mathcal{I}$ um ideal de operadores. Um polinômio $n$ homogêneo $P \in \mathcal{P}\left({ }^{n} E ; F\right)$ pertence a $\mathcal{I} \circ \mathcal{P}$, e neste caso escreveremos $P \in \mathcal{I} \circ \mathcal{P}\left({ }^{n} E ; F\right)$, se existem um espaço de Banach $G$, um polinômio $n$-homogêneo $Q \in \mathcal{P}\left({ }^{n} E ; G\right)$ e um operador $u \in \mathcal{I}(G ; F)$ tais que $P=u \circ Q$.

Proposição 1.6.14. [17, Proposition 3.2] Seja $\mathcal{I}$ um ideal de operadores. Então, para $P \in \mathcal{P}\left({ }^{n} E ; F\right)$, são equivalentes: 
(a) $P \in \mathcal{I} \circ \mathcal{P}\left({ }^{n} E ; F\right)$.

(b) $P_{L} \in \mathcal{I}\left(\widehat{\otimes}_{\pi}^{n, s} E ; F\right)$.

(c) $P_{L, s} \in \mathcal{I}\left(\widehat{\otimes}_{\pi_{s}}^{n, s} E ; F\right)$.

(d) $\check{P} \in \mathcal{I} \circ \mathcal{L}\left({ }^{n} E ; F\right)$.

(e) Existe $A \in \mathcal{I} \circ \mathcal{L}\left({ }^{n} E ; F\right)$ tal que $\widehat{A}=P$.

Definição 1.6.15. [17, Definition 3.6] Seja $\left(\mathcal{I},\|\cdot\|_{\mathcal{I}}\right)$ um ideal normado de operadores. Para cada $P \in \mathcal{I} \circ \mathcal{P}\left({ }^{n} E ; F\right)$, definimos

$$
\begin{gathered}
\|P\|_{\mathcal{I}_{\circ} \mathcal{P}, 1}:=\|\check{P}\|_{\mathcal{I} \circ \mathcal{L}} \text { e } \\
\|P\|_{\mathcal{I}_{\circ}, 2}:=\inf \left\{\|u\|_{\mathcal{I}} \cdot\|Q\|: P=u \circ Q, Q \in \mathcal{P}\left({ }^{n} E ; G\right), u \in \mathcal{I}(G ; F)\right\} .
\end{gathered}
$$

Proposição 1.6.16. [17, Proposition 3.7] $\operatorname{Seja}\left(\mathcal{I},\|\cdot\|_{\mathcal{I}}\right)$ um ideal normado (de Banach) de operadores. Então $\|\cdot\|_{\mathcal{I} \circ \mathcal{P}, 1}$ e $\|\cdot\|_{\mathcal{I} \circ \mathcal{P}, 2}$ tornam $\mathcal{I} \circ \mathcal{P}$ um ideal normado (de Banach) de polinômios. Além disso, para todo $P \in \mathcal{I} \circ \mathcal{P}\left({ }^{n} E ; F\right)$,

$$
\begin{gathered}
\|P\|_{\mathcal{I} \circ \mathcal{P}, 1}=\left\|P_{L}\right\|_{\mathcal{I}}=\inf \left\{\|A\|_{\mathcal{I} \circ \mathcal{L}}: A \in \mathcal{I} \circ \mathcal{L}\left({ }^{n} E ; F\right) e \check{A}=P\right\}, \\
\|P\|_{\mathcal{I} \circ \mathcal{P}, 2}=\left\|P_{L, s}\right\|_{\mathcal{I}} e\|P\|_{\mathcal{I} \circ \mathcal{P}, 2} \leq\|P\|_{\mathcal{I} \mathcal{P}, 1} \leq \frac{n^{n}}{n !}\|P\|_{\mathcal{I} \circ \mathcal{P}, 2} .
\end{gathered}
$$

Vejamos que algumas classes notáveis de polinômios são ideais de composição.

Um polinômio $P \in \mathcal{P}\left({ }^{n} E ; F\right)$ é compacto (fracamente compacto, respectivamente) se $P\left(B_{E}\right)$ é um subconjunto relativamente compacto (relativamente fracamente compacto, respectivamente) de $F$.

Proposição 1.6.17. ([66, Lemma 4.1] ou [56, Proposition 3.4]) Um polinômio $P \in$ $\mathcal{P}\left({ }^{n} E ; F\right)$ é fracamente compacto (respectivamente, compacto) se, e somente se, o operador linear $P_{L}: \widehat{\otimes}_{\pi}^{n, s} E \longrightarrow F$ é fracamente compacto (respectivamente, compacto).

Denotando por $\mathcal{P}_{\mathcal{K}}$ e $\mathcal{P}_{\mathcal{W}}$ as classes dos polinômios homogêneos compactos e fracamente compactos, as Proposições 1.6.16 e 1.6.17 garantem que

$$
\mathcal{P}_{\mathcal{K}}=\mathcal{K} \circ \mathcal{P} \text { e } \mathcal{P}_{\mathcal{W}}=\mathcal{W} \circ \mathcal{P}
$$

são ideais fechados de polinômios. 


\section{Capítulo 2}

\section{Operadores Multilineares Hiper-Nucleares}

Este capítulo tem dois objetivos principais: (i) provar alguns resultados novos, e que serão úteis na sequência, sobre operadores multilineares hiper- $(s ; r)$-nucleares, que foram introduzidos e desenvolvidos em [20,72]; (ii) introduzir e estudar a classe dos operadores hiper- $\sigma(p)$-nucleares, que é um hiper-ideal, ao contrário dos operadores multilineares $\sigma(p)$ nucleares estudados em [15], que formam um multi-ideal que não é um hiper-ideal. Todas essas classes generalizam e estendem os operadores $\sigma$-nucleares de Pietsch [59].

\subsection{Operadores multilineares hiper- $(s ; r)$-nucleares}

Em [20, 72] a classe dos operadores multilineares hiper-nucleares desempenha um papel de destaque por ser o menor hiper-ideal de Banach. Aqui, essa classe continuará tendo destaque, mas por uma outra razão que se esclarecerá no capítulo seguinte.

Além disso, os operadores multilineares hiper- $(s ; r)$-nucleares serão objeto de aplicações no próximo capítulo. Por este motivo, recordaremos nesta seção a definição e alguns resultados a respeito de tais operadores. Provaremos também alguns fatos que serão necessários e que ainda não haviam sido provados.

Definição 2.1.1. [72] Sejam $s \in[1, \infty)$ e $r \in[1, \infty]$ tais que $1 \leq \frac{1}{s}+\frac{1}{r}$. Um operador $n$ linear $A \in \mathcal{L}\left(E_{1}, \ldots, E_{n} ; F\right)$ é chamado hiper- $(s ; r)$-nuclear se existem sequências $\left(\lambda_{j}\right)_{j=1}^{\infty} \in$ 
$\ell_{s},\left(B_{j}\right)_{j=1}^{\infty} \in \ell_{r}^{w}\left(\mathcal{L}\left(E_{1}, \ldots, E_{n}\right)\right)$ e $\left(y_{j}\right)_{j=1}^{\infty} \in \ell_{\infty}(F)$ tais que

$$
A\left(x_{1}, \ldots, x_{n}\right)=\sum_{j=1}^{\infty} \lambda_{j} B_{j} \otimes y_{j}\left(x_{1}, \ldots, x_{n}\right)
$$

para todo $\left(x_{1}, \ldots, x_{n}\right) \in E_{1} \times \cdots \times E_{n}$. Neste caso dizemos que $\sum_{j=1}^{\infty} \lambda_{j} B_{j} \otimes y_{j}$ é uma representação hiper- $(s ; r)$-nuclear de $A$.

Denotamos a classe dos operadores hiper- $(s ; r)$-nucleares por $\mathcal{L}_{\mathcal{H N}_{(s ; r)}}$ e definimos a norma hiper- $(s ; r)$-nuclear por

$$
\|A\|_{\mathcal{H} \mathcal{N}_{(s ; r)}}=\inf \left\{\left\|\left(\lambda_{j}\right)_{j=1}^{\infty}\right\|_{s} \cdot\left\|\left(B_{j}\right)_{j=1}^{\infty}\right\|_{w, r} \cdot\left\|\left(y_{j}\right)_{j=1}^{\infty}\right\|_{\infty}\right\}
$$

onde o ínfimo é tomado sobre todas as representações hiper- $(s ; r)$-nucleares de $A$ na forma descrita acima.

Teorema 2.1.2. [72, Teorema 2.2.9] A classe $\left(\mathcal{L}_{\mathcal{H N}_{(s ; r)}},\|\cdot\|_{\mathcal{H N}_{(s ; r)}}\right)$ dos operadores multilineares hiper- $(s ; r)$-nucleares é um hiper-ideal de Banach.

Proposição 2.1.3. Seja $A \in \mathcal{L}_{\mathcal{H N}_{(s ; r)}}\left(E_{1}, \ldots, E_{n} ; F\right)$ com

$$
A=\sum_{j=1}^{\infty} \lambda_{j} B_{j} \otimes y_{j}
$$

uma representação hiper- $(s ; r)$-nuclear de A. Então a convergência em (2.1) se dá na norma $\|\cdot\|_{\mathcal{H N}_{(s ; r)}}$.

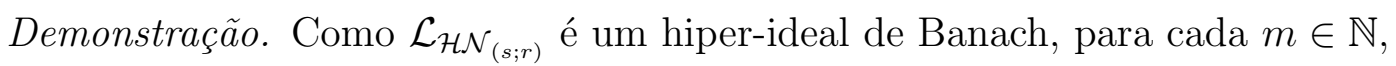

$$
\left(A-\sum_{j=1}^{m} \lambda_{j} B_{j} \otimes y_{j}\right) \in \mathcal{L}_{\mathcal{H} \mathcal{N}_{(s ; r)}}\left(E_{1}, \ldots, E_{n} ; F\right) .
$$

É claro que $\left\|\left(\lambda_{j}\right)_{j=m+1}^{\infty}\right\|_{s} \leq\left\|\left(\lambda_{j}\right)_{j=1}^{\infty}\right\|_{s},\left\|\left(B_{j}\right)_{j=m+1}^{\infty}\right\|_{w, r} \leq\left\|\left(B_{j}\right)_{j=1}^{\infty}\right\|_{w, r}$ e $\left\|\left(y_{j}\right)_{j=m+1}^{\infty}\right\|_{\infty} \leq$ $\left\|\left(y_{j}\right)_{j=1}^{\infty}\right\|_{\infty}$. Logo, $\sum_{j=m+1}^{\infty} \lambda_{j} B_{j} \otimes y_{j}$ é uma representação hiper- $(s ; r)$-nuclear de $A-$ $\sum_{j=1}^{m} \lambda_{j} B_{j} \otimes y_{j}$. Assim

$$
\left\|A-\sum_{j=1}^{m} \lambda_{j} B_{j} \otimes y_{j}\right\|_{\mathcal{H N}_{(s ; r)}}=\left\|\sum_{j=m+1}^{\infty} \lambda_{j} B_{j} \otimes y_{j}\right\|_{\mathcal{H N}_{(s ; r)}}
$$




$$
\begin{aligned}
& \leq\left\|\left(\lambda_{j}\right)_{j=m+1}^{\infty}\right\|_{s} \cdot\left\|\left(B_{j}\right)_{j=m+1}^{\infty}\right\|_{w, r} \cdot\left\|\left(y_{j}\right)_{j=m+1}^{\infty}\right\|_{\infty} \\
& \leq\left(\sum_{j=m+1}^{\infty}\left|\lambda_{j}\right|^{s}\right)^{\frac{1}{s}} \cdot\left\|\left(B_{j}\right)_{j=1}^{\infty}\right\|_{w, r} \cdot\left\|\left(y_{j}\right)_{j=1}^{\infty}\right\| .
\end{aligned}
$$

Como $\sum_{j=1}^{\infty}\left|\lambda_{j}\right|^{s}<+\infty$, fazendo $m \rightarrow \infty$ obtemos

$$
\left\|A-\sum_{j=1}^{m} \lambda_{j} B_{j} \otimes y_{j}\right\|_{\mathcal{H N}_{(s ; r)}} \longrightarrow 0
$$

provando que $A=\sum_{j=1}^{\infty} \lambda_{j} B_{j} \otimes y_{j}$ na norma $\|\cdot\|_{\mathcal{H N}_{(s ; r)}}$.

O resultado a seguir é bem conhecido.

Proposição 2.1.4. [72, Proposição 2.1.6] Seja $\left(\mathcal{H},\|\cdot\|_{\mathcal{H}}\right)$ um multi-ideal normado. Então $\|\cdot\| \leq\|\cdot\|_{\mathcal{H}}$.

Em particular, $\|\cdot\| \leq\|\cdot\|_{\mathcal{H N}}(s ; r)$

O resultado que demonstramos a seguir mostra que o hiper-ideal dos operadores hiper$(s ; r)$-nucleares é um ideal de composição, cuja componente linear é o ideal dos operadores lineares $(s ; r)$-nucleares visto na Definição 1.4.6.

Proposição 2.1.5. Sejam $E_{1}, \ldots, E_{n}$ e F espaços de Banach. Então

$$
\mathcal{L}_{\mathcal{H N}_{(s ; r)}}\left(E_{1}, \ldots, E_{n} ; F\right)=\mathcal{N}_{(s ; r)} \circ \mathcal{L}\left(E_{1}, \ldots, E_{n} ; F\right)
$$

$e\|\cdot\|_{\mathcal{H} \mathcal{N}_{(s ; r)}}=\|\cdot\|_{\mathcal{N}_{(s ; r)} \circ \mathcal{L}}$.

Demonstração. Seja $A \in \mathcal{N}_{(s ; r)} \circ \mathcal{L}\left(E_{1}, \ldots, E_{n} ; F\right)$. Existem um espaço de Banach $G$ e operadores $B \in \mathcal{L}\left(E_{1}, \ldots, E_{n} ; G\right)$ e $u \in \mathcal{N}_{(s ; r)}(G ; F)$ tais que $A=u \circ B$.

Dado $\varepsilon>0$, como $u \in \mathcal{N}_{(s ; r)}(G ; F)$, podemos tomar $\left(\lambda_{j}\right)_{j=1}^{\infty} \in \ell_{s},\left(y_{j}\right)_{j=1}^{\infty} \in \ell_{\infty}(F) \mathrm{e}$ $\left(\varphi_{j}\right)_{j=1}^{\infty} \in \ell_{r}^{w}\left(G^{*}\right)$ tais que $u=\sum_{j=1}^{\infty} \lambda_{j} \varphi_{j} \otimes y_{j} \mathrm{e}$

$$
\left\|\left(\lambda_{j}\right)_{j=1}^{\infty}\right\|_{s} \cdot\left\|\left(\varphi_{j}\right)_{j=1}^{\infty}\right\|_{w, r} \cdot\left\|\left(y_{j}\right)_{j=1}^{\infty}\right\|_{\infty} \leq(1+\varepsilon)\|u\|_{\mathcal{N}_{(s ; r)}} .
$$

De $A=u \circ B$, segue que

$$
A\left(x_{1}, \ldots, x_{n}\right)=u\left(B\left(x_{1}, \ldots, x_{n}\right)\right)=\sum_{j=1}^{\infty} \lambda_{j} \varphi_{j}\left(B\left(x_{1}, \ldots, x_{n}\right)\right) y_{j}
$$




$$
=\sum_{j=1}^{\infty} \lambda_{j}\left(\varphi_{j} \circ B\right) \otimes y_{j}\left(x_{1}, \ldots, x_{n}\right)
$$

para qualquer $\left(x_{1}, \ldots, x_{n}\right) \in E_{1} \times \cdots \times E_{n}$. Vejamos que $A=\sum_{j=1}^{\infty} \lambda_{j}\left(\varphi_{j} \circ B\right) \otimes y_{j}$ é uma representação hiper- $(s ; r)$-nuclear de $A$. Com efeito, já sabemos que $\left(\lambda_{j}\right)_{j=1}^{\infty} \in \ell_{s}$ e $\left(y_{j}\right)_{j=1}^{\infty} \in \ell_{\infty}(F)$. Resta então verificar que $\left(\varphi_{j} \circ B\right)_{j=1}^{\infty} \in \ell_{r}^{w}\left(\mathcal{L}\left(E_{1}, \ldots, E_{n}\right)\right)$. Assim como no caso do adjunto de Aron-Schottenloher [6] de um polinômio homogêneo, é fácil ver que o adjunto de $B$,

$$
B^{*}: G^{*} \longrightarrow \mathcal{L}\left(E_{1}, \ldots, E_{n}\right), B^{*}(\varphi)\left(x_{1}, \ldots, x_{n}\right)=\varphi\left(B\left(x_{1}, \ldots, x_{n}\right)\right),
$$

é um operador linear contínuo. Mais ainda, pelo Teorema de Hahn-Banach,

$$
\begin{aligned}
\left\|B^{*}\right\| & =\sup _{\psi \in B_{G^{*}}} \sup _{x_{j} \in B_{E_{j}}}\left|B^{*}(\psi)\left(x_{1}, \ldots, x_{n}\right)\right|=\sup _{\psi \in B_{G^{*}}} \sup _{x_{j} \in B_{E_{j}}}\left|\psi\left(B\left(x_{1}, \ldots, x_{n}\right)\right)\right| \\
& =\sup _{x_{j} \in B_{E_{j}}} \sup _{\psi \in B_{G^{*}}}\left|\psi\left(B\left(x_{1}, \ldots, x_{n}\right)\right)\right|=\sup _{x_{j} \in B_{E_{j}}}\left\|B\left(x_{1}, \ldots, x_{n}\right)\right\|=\|B\| .
\end{aligned}
$$

Para a validade da inversão dos supremos, veja [45, Lema 2.4.10]. Como $\left(\varphi_{j}\right)_{j=1}^{\infty} \in \ell_{r}^{w}\left(G^{*}\right)$, pela Proposição 1.1 .9 (ii), segue que

$$
\left(\varphi_{j} \circ B\right)_{j=1}^{\infty}=\left(B^{*}\left(\varphi_{j}\right)\right)_{j=1}^{\infty} \in \ell_{r}^{w}\left(\mathcal{L}\left(E_{1}, \ldots, E_{n}\right)\right)
$$

e

$$
\left\|\left(\varphi_{j} \circ B\right)_{j=1}^{\infty}\right\|_{w, r}=\left\|\left(B^{*}\left(\varphi_{j}\right)\right)_{j=1}^{\infty}\right\|_{w, r} \leq\left\|B^{*}\right\| \cdot\left\|\left(\varphi_{j}\right)_{j=1}^{\infty}\right\|_{w, r}=\|B\| \cdot\left\|\left(\varphi_{j}\right)_{j=1}^{\infty}\right\|_{w, r} .
$$

Então $A=\sum_{j=1}^{\infty} \lambda_{j}\left(\varphi_{j} \circ B\right) \otimes y_{j} \in \mathcal{L}_{\mathcal{H N}_{(s ; r)}}\left(E_{1}, \ldots, E_{n} ; F\right) \mathrm{e}$

$$
\begin{aligned}
\|A\|_{\mathcal{H} \mathcal{N}_{(s ; r)}} & \leq\left\|\left(\lambda_{j}\right)_{j=1}^{\infty}\right\|_{s} \cdot\left\|\left(\varphi_{j} \circ B\right)_{j=1}^{\infty}\right\|_{w, r} \cdot\left\|\left(y_{j}\right)_{j=1}^{\infty}\right\|_{\infty} \\
& \leq\left\|\left(\lambda_{j}\right)_{j=1}^{\infty}\right\|_{s} \cdot\|B\| \cdot\left\|\left(\varphi_{j}\right)_{j=1}^{\infty}\right\|_{w, r} \cdot\left\|\left(y_{j}\right)_{j=1}^{\infty}\right\|_{\infty} \\
& \leq\|B\| \cdot(1+\varepsilon)\|u\|_{\mathcal{N}_{(s ; r)}} .
\end{aligned}
$$

Fazendo $\varepsilon \rightarrow 0$, obtemos $\|A\|_{\mathcal{H N}_{(s ; r)}} \leq\|B\| \cdot\|u\|_{\mathcal{N}_{(s ; r)}}$, para toda fatoração $A=u \circ B$ com $u \in \mathcal{N}_{(s ; r)}(G ; F)$. Tomando o ínfimo sobre todas essas fatorações de $A$ segue que

$$
\|A\|_{\mathcal{H \mathcal { N }}(s ; r)} \leq\|A\|_{\mathcal{N}_{(s ; r)} \circ \mathcal{L}} .
$$

Reciprocamente, dados $A \in \mathcal{L}_{\mathcal{H N}_{(s ; r)}}\left(E_{1}, \ldots, E_{n} ; F\right)$ e $\varepsilon>0$, existem $\left(\lambda_{j}\right)_{j=1}^{\infty} \in \ell_{s}$, $\left(B_{j}\right)_{j=1}^{\infty} \in \ell_{r}^{w}\left(\mathcal{L}\left(E_{1}, \ldots, E_{n}\right)\right)$ e $\left(y_{j}\right)_{j=1}^{\infty} \in \ell_{\infty}(F)$ tais que $A=\sum_{j=1}^{\infty} \lambda_{j} B_{j} \otimes y_{j} \mathrm{e}$

$$
\left\|\left(\lambda_{j}\right)_{j=1}^{\infty}\right\|_{s} \cdot\left\|\left(B_{j}\right)_{j=1}^{\infty}\right\|_{w, r} \cdot\left\|\left(y_{j}\right)_{j=1}^{\infty}\right\|_{\infty} \leq(1+\varepsilon)\|A\|_{\mathcal{H N}(s ; r)} .
$$


Pela Proposição 1.4 .8 é suficiente mostrarmos que $A_{L} \in \mathcal{N}_{(s ; r)}\left(E_{1} \widehat{\otimes}_{\pi} \cdots \widehat{\otimes}_{\pi} E_{n} ; F\right)$. Pela Proposição 2.1.3 sabemos que a convergência $A=\sum_{j=1}^{\infty} \lambda_{j} B_{j} \otimes y_{j}$ se dá na norma hiper$(s ; r)$-nuclear, logo, pela Proposição 2.1.4, essa convergência também se dá na norma usual de operadores multilineares. Temos então $A=\lim _{m \rightarrow \infty} \sum_{j=1}^{m} \lambda_{j} B_{j} \otimes y_{j}$ em $\mathcal{L}\left(E_{1}, \ldots, E_{n} ; F\right)$. Sendo o operador linearização

$$
B \in \mathcal{L}\left(E_{1}, \ldots, E_{n} ; F\right) \mapsto B_{L} \in \mathcal{L}\left(E_{1} \widehat{\otimes}_{\pi} \cdots \widehat{\otimes}_{\pi} E_{n} ; F\right)
$$

um isomorfismo isométrico (Teorema 1.2.13), de sua linearidade e de sua continuidade segue que

$$
\begin{aligned}
A_{L}=\left(\lim _{m \rightarrow \infty} \sum_{j=1}^{m} \lambda_{j} B_{j} \otimes y_{j}\right)_{L} & =\lim _{m \rightarrow \infty} \sum_{j=1}^{m} \lambda_{j}\left(B_{j} \otimes y_{j}\right)_{L} \\
& =\lim _{m \rightarrow \infty} \sum_{j=1}^{m} \lambda_{j}\left(B_{j}\right)_{L} \otimes y_{j}=\sum_{j=1}^{\infty} \lambda_{j}\left(B_{j}\right)_{L} \otimes y_{j}
\end{aligned}
$$

em $\mathcal{L}\left(E_{1} \widehat{\otimes}_{\pi} \cdots \widehat{\otimes}_{\pi} E_{n} ; F\right)$. Mostremos que

$$
A_{L}=\sum_{j=1}^{\infty} \lambda_{j}\left(B_{j}\right)_{L} \otimes y_{j}
$$

é uma representação $(s ; r)$-nuclear para $A_{L}$. Com efeito, já temos $\left(\lambda_{j}\right)_{j=1}^{\infty} \in \ell_{s}$ e $\left(y_{j}\right)_{j=1}^{\infty} \in$ $\ell_{\infty}(F)$. Usando mais uma vez que o operador linearização

$$
L: \mathcal{L}\left(E_{1}, \ldots, E_{n}\right) \longrightarrow\left(E_{1} \widehat{\otimes}_{\pi} \cdots \widehat{\otimes}_{\pi} E_{n}\right)^{*}, L(B)=B_{L},
$$

é um isomorfismo isométrico, como $\left(B_{j}\right)_{j=1}^{\infty} \in \ell_{r}^{w}\left(\mathcal{L}\left(E_{1}, \ldots, E_{n}\right)\right)$, da Proposição 1.1.9 decorre que

$$
L\left(\left(B_{j}\right)_{j=1}^{\infty}\right)=\left(\left(B_{j}\right)_{L}\right)_{j=1}^{\infty} \in \ell_{r}^{w}\left(\left(E_{1} \widehat{\otimes}_{\pi} \cdots \widehat{\otimes}_{\pi} E_{n}\right)^{*}\right)
$$

e

$$
\left\|\left(\left(B_{j}\right)_{L}\right)_{j=1}^{\infty}\right\|_{w, r} \leq\|L\| \cdot\left\|\left(B_{j}\right)_{j=1}^{\infty}\right\|_{w, r}=\left\|\left(B_{j}\right)_{j=1}^{\infty}\right\|_{w, r} .
$$

Portanto, $A_{L} \in \mathcal{N}_{(s ; r)}\left(E_{1} \widehat{\otimes}_{\pi} \cdots \widehat{\otimes}_{\pi} E_{n} ; F\right)$ e

$$
\begin{aligned}
\left\|A_{L}\right\|_{\mathcal{N}_{(s ; r)}} & \leq\left\|\left(\lambda_{j}\right)_{j=1}^{\infty}\right\|_{s} \cdot\left\|\left(\left(B_{j}\right)_{L}\right)_{j=1}^{\infty}\right\|_{w, r} \cdot\left\|\left(y_{j}\right)_{j=1}^{\infty}\right\|_{\infty} \\
& \leq(1+\varepsilon)\|A\|_{\mathcal{H} \mathcal{N}_{(s ; r)}} .
\end{aligned}
$$

Fazendo $\varepsilon \rightarrow 0$, obtemos $\left\|A_{L}\right\|_{\mathcal{N}_{(s ; r)}} \leq\|A\|_{\mathcal{H N}_{(s ; r)}}$. Segue da Proposição 1.4 .9 que

$$
\|A\|_{\mathcal{N}_{(s ; r)} \circ \mathcal{L}} \leq\|A\|_{\mathcal{H} \mathcal{N}_{(s ; r)}} .
$$


Tomando $s=1$ e $r=\infty$ na Definição 2.1.1, obtemos o hiper-ideal de Banach dos operadores multilineares hiper- $(1 ; \infty)$-nucleares, que serão chamados simplesmente de operadores hiper-nucleares. Denotaremos esse ideal, que desempenhará papel central no próximo capítulo, por $\mathcal{L}_{\mathcal{H N}}$ ao invés de $\mathcal{L}_{\mathcal{H N}}(1 ; \infty)$.

Como caso particular da proposição acima, obtemos o

Corolário 2.1.6. $\mathcal{L}_{\mathcal{H N}}=\mathcal{N} \circ \mathcal{L}$ isometricamente.

A seguinte fórmula para a norma hiper-nuclear de um operador multilinear também será útil no próximo capítulo.

Lema 2.1.7. [72, Lema 2.2.11] Para todo operador $n$-linear $A \in \mathcal{L}_{\mathcal{H N}}\left(E_{1}, \ldots, E_{n} ; F\right)$, vale que

$$
\|A\|_{\mathcal{H N}}=\inf \left\{\sum_{j=1}^{\infty}\left|\lambda_{j}\right| \cdot\left\|B_{j}\right\| \cdot\left\|y_{j}\right\|\right\}
$$

onde o ínfimo é tomado sobre todas as representações hiper-nucleares $\sum_{j=1}^{\infty} \lambda_{j} B_{j} \otimes y_{j}$ de $A$.

\section{$2.2 \quad$ Operadores multilineares hiper- $\sigma(p)$-nucleares}

A classe dos operadores multilineares $\sigma(p)$-nucleares foi introduzida em [15] (veja também [16]), como uma generalização linear e multilinear dos operadores lineares $\sigma$ nucleares estudados por Pietsch [59]. Essa classe é um multi-ideal que não é um hiperideal. Introduziremos nesta seção uma variante desse multi-ideal, formada pelos operadores multilineares hiper- $\sigma(p)$-nucleares, que se apresenta naturalmente no âmbito dos hiper-ideais. A ideia é substituir os produtos de avaliações em funcionais lineares por avaliações em formas multilineares.

Definição 2.2.1. Seja $1 \leq p<\infty$. Dizemos que um operador $n$-linear $A \in \mathcal{L}\left(E_{1}, \ldots, E_{n} ; F\right)$ é hiper- $\sigma(p)$-nuclear se existem sequências $\left(\lambda_{j}\right)_{j=1}^{\infty} \in \ell_{p^{*}},\left(B_{j}\right)_{j=1}^{\infty} \subseteq \mathcal{L}\left(E_{1}, \ldots, E_{n}\right)$ e $\left(y_{j}\right)_{j=1}^{\infty} \subseteq F$ tais que:

(i) $A\left(x_{1}, \ldots, x_{n}\right)=\sum_{j=1}^{\infty} \lambda_{j} B_{j} \otimes y_{j}\left(x_{1}, \ldots, x_{n}\right)=\sum_{j=1}^{\infty} \lambda_{j} B_{j}\left(x_{1}, \ldots, x_{n}\right) y_{j}$, para todo $\left(x_{1}, \ldots, x_{n}\right) \in$ $E_{1} \times \cdots \times E_{n}$.

(ii) $\sup _{x_{i} \in B_{E_{i}}, y^{*} \in B_{F^{*}}}\left(\sum_{j=1}^{\infty}\left|B_{j}\left(x_{1}, \ldots, x_{n}\right) y^{*}\left(y_{j}\right)\right|^{p}\right)^{\frac{1}{p}}<\infty$. 
(iii) $\lim _{m \rightarrow \infty} \sup _{x_{i} \in B_{E_{i}}, y^{*} \in B_{F^{*}}}\left(\sum_{j=m}^{\infty}\left|B_{j}\left(x_{1}, \ldots, x_{n}\right) y^{*}\left(y_{j}\right)\right|^{p}\right)^{\frac{1}{p}}=0$.

Neste caso, escreveremos $A \in \mathcal{L}_{\mathcal{H} \sigma(p)}\left(E_{1}, \ldots, E_{n} ; F\right)$ e dizemos que $A=\sum_{j=1}^{\infty} \lambda_{j} B_{j} \otimes y_{j}$ é uma representação hiper- $\sigma(p)$-nuclear de $A$.

Definimos ainda $\|\cdot\|_{\mathcal{H} \sigma(p)}: \mathcal{L}_{\mathcal{H} \sigma(p)}\left(E_{1}, \ldots, E_{n} ; F\right) \longrightarrow[0, \infty)$ por

$$
\|A\|_{\mathcal{H} \sigma(p)}=\inf \left\{\left\|\left(\lambda_{j}\right)_{j=1}^{\infty}\right\|_{p^{*}} \cdot \sup _{x_{i} \in B_{E_{i}}, y^{*} \in B_{F^{*}}}\left(\sum_{j=1}^{\infty}\left|B_{j}\left(x_{1}, \ldots, x_{n}\right) y^{*}\left(y_{j}\right)\right|^{p}\right)^{\frac{1}{p}}\right\},
$$

onde o ínfimo é tomado sobre todas as representações hiper- $\sigma(p)$-nucleares $\sum_{j=1}^{\infty} \lambda_{j} B_{j} \otimes y_{j}$ de $A$.

O caso $n=p=1$ recupera os operadores lineares $\sigma$-nucleares de Pietsch [59], e o caso $n=1$ recupera os operadores lineares $\sigma(p)$-nucleares do Exemplo 1.4.4.

Começamos o estudo da classe dos operadores hiper- $\sigma(p)$-nucleares com dois fatos básicos sobre $\|\cdot\|_{\mathcal{H} \sigma(p)}$.

Lema 2.2.2. $\|A\| \leq\|A\|_{\mathcal{H} \sigma(p)}$ para todo operador $A \in \mathcal{L}_{\mathcal{H} \sigma(p)}\left(E_{1}, \ldots, E_{n} ; F\right)$.

Demonstração. Dado $\varepsilon>0$, podemos tomar uma representação hiper- $\sigma(p)$-nuclear $A=$ $\sum_{j=1}^{\infty} \lambda_{j} B_{j} \otimes y_{j}$ tal que

$$
\left\|\left(\lambda_{j}\right)_{j=1}^{\infty}\right\|_{p^{*}} \cdot \sup _{x_{i} \in B_{E_{i}}, y^{*} \in B_{F^{*}}}\left(\sum_{j=1}^{\infty}\left|B_{j}\left(x_{1}, \ldots, x_{n}\right) y^{*}\left(y_{j}\right)\right|^{p}\right)^{\frac{1}{p}} \leq(1+\varepsilon)\|A\|_{\mathcal{H} \sigma(p)} .
$$

Aplicando primeiro o Teorema de Hahn-Banach e depois a Desigualdade de Hölder, obtemos

$$
\begin{aligned}
\|A\| & =\sup _{x_{i} \in B_{E_{i}}}\left\|A\left(x_{1}, \ldots, x_{n}\right)\right\|=\sup _{x_{i} \in B_{E_{i}}} \sup _{y^{*} \in B_{F^{*}}}\left|y^{*}\left(A\left(x_{1}, \ldots, x_{n}\right)\right)\right| \\
& =\sup _{x_{i} \in B_{E_{i}}, y^{*} \in B_{F^{*}}}\left|y^{*}\left(\sum_{j=1}^{\infty} \lambda_{j} B_{j}\left(x_{1}, \ldots, x_{n}\right) y_{j}\right)\right| \\
& =\sup _{x_{i} \in B_{E_{i}}, y^{*} \in B_{F^{*}}}\left|\sum_{j=1}^{\infty} \lambda_{j} B_{j}\left(x_{1}, \ldots, x_{n}\right) y^{*}\left(y_{j}\right)\right|
\end{aligned}
$$




$$
\begin{aligned}
& \leq \sup _{x_{i} \in B_{E_{i}}, y^{*} \in B_{F^{*}}} \sum_{j=1}^{\infty}\left|\lambda_{j}\right| \cdot\left|B_{j}\left(x_{1}, \ldots, x_{n}\right) y^{*}\left(y_{j}\right)\right| \\
& \leq \sup _{x_{i} \in B_{E_{i}}, y^{*} \in B_{F^{*}}}\left(\sum_{j=1}^{\infty}\left|\lambda_{j}\right|^{p^{*}}\right)^{\frac{1}{p^{*}}} \cdot\left(\sum_{j=1}^{\infty}\left|B_{j}\left(x_{1}, \ldots, x_{n}\right) y^{*}\left(y_{j}\right)\right|^{p}\right)^{\frac{1}{p}} \\
& =\left\|\left(\lambda_{j}\right)_{j=1}^{\infty}\right\|_{p^{*}} \cdot \sup _{x_{i} \in B_{E_{i}}, y^{*} \in B_{F^{*}}}\left(\sum_{j=1}^{\infty}\left|B_{j}\left(x_{1}, \ldots, x_{n}\right) y^{*}\left(y_{j}\right)\right|^{p}\right)^{\frac{1}{p}} \\
& \leq(1+\varepsilon)\|A\|_{\mathcal{H} \sigma(p)} .
\end{aligned}
$$

A desigualdade desejada segue fazendo $\varepsilon \longrightarrow 0^{+}$.

Ainda não vimos que $\mathcal{L}_{\mathcal{H} \sigma(p)}\left(E_{1}, \ldots, E_{n} ; F\right)$ é um espaço vetorial e nem que $\|\cdot\|_{\mathcal{H} \sigma(p)}$ é uma norma. De toda forma, é conveniente deixar demonstrado que uma representação hiper- $\sigma(p)$-nuclear converge tanto em relação a $\|\cdot\|_{\mathcal{H} \sigma(p)}$ como em relação à norma usual $\|\cdot\|$.

Proposição 2.2.3. Sejam $A \in \mathcal{L}_{\mathcal{H} \sigma(p)}\left(E_{1}, \ldots, E_{n} ; F\right) e$

$$
A=\sum_{j=1}^{\infty} \lambda_{j} A_{j} \otimes y_{j}
$$

uma representação hiper- $\sigma(p)$-nuclear para $A$. Então, para todo $m \in \mathbb{N}$,

$$
\left(A-\sum_{j=1}^{m} \lambda_{j} A_{j} \otimes y_{j}\right) \in \mathcal{L}_{\mathcal{H} \sigma(p)}\left(E_{1}, \ldots, E_{n} ; F\right)
$$

$e$

$$
\lim _{m \rightarrow \infty}\left\|A-\sum_{j=1}^{m} \lambda_{j} A_{j} \otimes y_{j}\right\|_{\mathcal{H} \sigma(p)}=0 .
$$

Em particular, a convergência em (2.3) se dá na norma usual de $\mathcal{L}\left(E_{1}, \ldots, E_{n} ; F\right)$.

Demonstração. Seja $m \in \mathbb{N}$. Para $x_{1} \in E_{1}, \ldots, x_{n} \in E_{n}$,

$$
\begin{aligned}
\left(A-\sum_{j=1}^{m} \lambda_{j} A_{j} \otimes y_{j}\right)\left(x_{1}, \ldots, x_{n}\right) & =\sum_{j=1}^{\infty} \lambda_{j} A_{j}\left(x_{1}, \ldots, x_{n}\right) y_{j}-\sum_{j=1}^{m} \lambda_{j} A_{j}\left(x_{1}, \ldots, x_{n}\right) y_{j} \\
& =\sum_{j=m+1}^{\infty} \lambda_{j} A_{j}\left(x_{1}, \ldots, x_{n}\right) y_{j} .
\end{aligned}
$$


É claro que $\left\|\left(\lambda_{j}\right)_{j=m+1}^{\infty}\right\|_{p^{*}} \leq\left\|\left(\lambda_{j}\right)_{j=1}^{\infty}\right\|_{p^{*}}$, e portanto $\left(\lambda_{j}\right)_{j=m+1}^{\infty} \in \ell_{p^{*}}$. Mais ainda, $\sup _{x_{i} \in B_{E_{i}}, y^{*} \in B_{F^{*}}} \sum_{j=m+1}^{\infty}\left|B_{j}\left(x_{1}, \ldots, x_{n}\right) y^{*}\left(y_{j}\right)\right|^{p} \leq \sup _{x_{i} \in B_{E_{i}}, y^{*} \in B_{F^{*}}} \sum_{j=1}^{\infty}\left|B_{j}\left(x_{1}, \ldots, x_{n}\right) y^{*}\left(y_{j}\right)\right|^{p}<\infty$. Combinando tudo isso com a condição (iii) da Definição 2.2.1 concluímos que $\sum_{j=m+1}^{\infty} \lambda_{j} A_{j} \otimes$ $y_{j}$ é uma representação hiper- $\sigma(p)$-nuclear de $A-\sum_{j=1}^{m} \lambda_{j} A_{j} \otimes y_{j}$, o que comprova a primeira afirmação.

Aplicando a definição de $\|\cdot\|_{\mathcal{H} \sigma(p)}$, a segunda afirmação segue de

$$
\begin{aligned}
\left\|A-\sum_{j=1}^{m} \lambda_{j} A_{j} \otimes y_{j}\right\|_{\mathcal{H} \sigma(p)} & =\left\|\sum_{j=m+1}^{\infty} \lambda_{j} A_{j} \otimes y_{j}\right\|_{\mathcal{H} \sigma(p)} \\
& \leq\left(\sum_{j=m+1}^{\infty}\left|\lambda_{j}\right|^{p^{*}}\right)^{\frac{1}{p^{*}}} \cdot \sup _{x_{i} \in B_{E_{i}}, y^{*} \in B_{F^{*}}}\left(\sum_{j=m+1}^{\infty}\left|A_{j}\left(x_{1}, \ldots, x_{n}\right) y^{*}\left(y_{j}\right)\right|^{p}\right)^{\frac{1}{p}} \\
& \leq\left(\sum_{j=m+1}^{\infty}\left|\lambda_{j}\right|^{p^{*}}\right)^{\frac{1}{p^{*}}} \cdot \underbrace{\sup _{x_{i} \in B_{E_{i}}, y^{*} \in B_{F^{*}}}\left(\sum_{j=1}^{\infty}\left|A_{j}\left(x_{1}, \ldots, x_{n}\right) y^{*}\left(y_{j}\right)\right|^{p}\right)^{\frac{1}{p}}}_{:=K<\infty} \\
& =K \cdot\left(\sum_{j=m+1}^{\infty}\left|\lambda_{j}\right|^{p^{*}}\right)^{\stackrel{\frac{1}{p^{*}}}{m_{m \rightarrow \infty}^{\longrightarrow}} 0,}
\end{aligned}
$$

uma vez que $\sum_{j=1}^{\infty}\left|\lambda_{j}\right|^{p^{*}}<\infty$

Combinando o que acabamos de provar com o Lema 2.2.2, obtemos

$$
\left\|A-\sum_{j=1}^{m} \lambda_{j} A_{j} \otimes y_{j}\right\| \leq\left\|A-\sum_{j=1}^{m} \lambda_{j} A_{j} \otimes y_{j}\right\|_{\mathcal{H} \sigma(p)} \stackrel{m \rightarrow \infty}{\longrightarrow} 0,
$$

o que comprova a última afirmação.

Veremos a seguir que essa nova classe $\mathcal{L}_{\mathcal{H} \sigma(p)}$ não é muito pequena nem muito grande, no sentido de que contém a classe $\mathcal{L}_{\mathcal{F}}$ dos operadores de posto finito e está contida em seu fecho $\overline{\mathcal{L}_{\mathcal{F}}}$. Mostraremos também que $\mathcal{L}_{\mathcal{H} \sigma(p)}$ contém propriamente a classe $\mathcal{L}_{\sigma(p)}$ dos operadores multilineares $\sigma(p)$-nucleares da Definição 1.4.4. Para isso precisamos do seguinte lema, a respeito desta classe. 
Lema 2.2.4. Para todos espaços de Banach $E_{1}, \ldots, E_{n}$ e $F$,

$$
\mathcal{L}_{f}\left(E_{1}, \ldots, E_{n} ; F\right) \subseteq \mathcal{L}_{\sigma(p)}\left(E_{1}, \ldots, E_{n} ; F\right) \subseteq \overline{\mathcal{L}_{f}}\left(E_{1}, \ldots, E_{n} ; F\right)
$$

Demonstração. A primeira inclusão é óbvia pela definição de operadores multilineares $\sigma(p)$-nucleares.

Dado $A \in \mathcal{L}_{\sigma(p)}\left(E_{1}, \ldots, E_{n} ; F\right)$, existem sequências $\left(\lambda_{j}\right)_{j=1}^{\infty} \in \ell_{p^{*}},\left(\varphi_{i_{j}}\right)_{j=1}^{\infty} \subseteq E_{i}^{*}$, $i=1, \ldots, n$, e $\left(y_{j}\right)_{j=1}^{\infty} \subseteq F$ tais que

$$
A=\sum_{j=1}^{\infty} \lambda_{j} \varphi_{1_{j}} \otimes \cdots \otimes \varphi_{n_{j}} \otimes y_{j}
$$

Como $\mathcal{L}_{\sigma(p)}\left(E_{1}, \ldots, E_{n} ; F\right)$ é espaço vetorial, $A-\sum_{j=1}^{m} \lambda_{j} \varphi_{1_{j}} \otimes \cdots \otimes \varphi_{n_{j}} \otimes y_{j}$ pertence a $\mathcal{L}_{\sigma(p)}\left(E_{1}, \ldots, E_{n} ; F\right)$ para todo $m$ e segue facilmente da definição que

$$
A-\sum_{j=1}^{m} \lambda_{j} \varphi_{1_{j}} \otimes \cdots \otimes \varphi_{n_{j}} \otimes y_{j}=\sum_{j=m+1}^{\infty} \lambda_{j} \varphi_{1_{j}} \otimes \cdots \otimes \varphi_{n_{j}} \otimes y_{j}
$$

é uma representação $\sigma(p)$-nuclear. Dessa forma,

$$
\begin{aligned}
\| A-\sum_{j=1}^{m} \lambda_{j} \varphi_{1_{j}} \otimes \cdots & \otimes \varphi_{n_{j}} \otimes y_{j}\left\|_{\sigma(p)}=\right\| \sum_{j=m+1}^{\infty} \lambda_{j} \varphi_{1_{j}} \otimes \cdots \otimes \varphi_{n_{j}} \otimes y_{j} \|_{\sigma(p)} \\
& \leq\left\|\left(\lambda_{j}\right)_{j=m+1}^{\infty}\right\|_{p^{*}} \cdot \sup _{x_{i} \in B_{E_{i}}, y^{*} \in B_{F^{*}}}\left(\sum_{j=m+1}^{\infty}\left|\varphi_{1_{j}}\left(x_{1}\right) \cdots \varphi_{n_{j}}\left(x_{n}\right) y^{*}\left(y_{j}\right)\right|^{p}\right)^{\frac{1}{p}} \\
& \leq\left\|\left(\lambda_{j}\right)_{j=m+1}^{\infty}\right\|_{p^{*}} \cdot \underbrace{\sup _{x_{i} \in B_{E_{i}}, y^{*} \in B_{F^{*}}}\left(\sum_{j=1}^{\infty} \mid \varphi_{1_{j}}\left(x_{1}\right) \cdots \varphi_{n_{j}}\left(x_{n}\right) y^{*}\left(y_{j}\right)^{p}\right)^{\frac{1}{p}}}_{:=K<\infty} \\
& =K \cdot\left(\sum_{j=m+1}^{\infty}\left|\lambda_{j}\right|^{p^{*}}\right)^{\frac{1}{p^{*}}} \stackrel{m \rightarrow \infty}{\longrightarrow} 0,
\end{aligned}
$$

pois $\left(\lambda_{j}\right)_{j=1}^{\infty} \in \ell_{p^{*}}$. Isso significa que a convergência $A=\sum_{j=1}^{\infty} \lambda_{j} \varphi_{1_{j}} \otimes \cdots \otimes \varphi_{n_{j}} \otimes y_{j}$ ocorre na norma $\sigma(p)$-nuclear em $\mathcal{L}_{\sigma(p)}\left(E_{1}, \ldots, E_{n} ; F\right)$. Por $\mathcal{L}_{\sigma(p)}$ ser um multi-ideal, vale que $\|\cdot\| \leq\|\cdot\|_{\sigma(p)}$, e daí concluímos que a sequência

$$
\left(\sum_{j=1}^{m} \lambda_{j} \varphi_{1_{j}} \otimes \cdots \otimes \varphi_{n_{j}} \otimes y_{j}\right)_{m=1}^{\infty}
$$


de operadores de tipo finito converge para $A$ na norma usual de operadores, ou seja, mostramos que $A$ é limite, na norma usual, de uma sequência de operadores de tipo finito e, portanto, $A \in \overline{\mathcal{L}_{f}}\left(E_{1}, \ldots, E_{n} ; F\right)$.

Teorema 2.2.5. Seja $1 \leq p<\infty$. Então:

(a) $\mathcal{L}_{\mathcal{F}} \subseteq \mathcal{L}_{\mathcal{H} \sigma(p)} \subseteq \overline{\mathcal{L}_{\mathcal{F}}}$.

(b) $\mathcal{L}_{\sigma(p)} \subseteq \mathcal{L}_{\mathcal{H} \sigma(p)}$ com $\|\cdot\|_{\mathcal{H} \sigma(p)} \leq\|\cdot\|_{\sigma(p)}$ e, em geral,

$$
\mathcal{L}_{\sigma(p)}\left(E_{1}, \ldots, E_{n} ; F\right) \neq \mathcal{L}_{\mathcal{H} \sigma(p)}\left(E_{1}, \ldots, E_{n} ; F\right) .
$$

Demonstração. (a) Para a primeira inclusão seja $A \in \mathcal{L}_{\mathcal{F}}\left(E_{1}, \ldots, E_{n} ; F\right)$. Existem $k \in \mathbb{N}$, formas $n$-lineares $B_{1}, \ldots, B_{k} \in \mathcal{L}\left(E_{1}, \ldots, E_{n}\right)$ e vetores $y_{1}, \ldots, y_{k} \in F$ tais que

$$
A\left(x_{1}, \ldots, x_{n}\right)=\sum_{j=1}^{k} B_{j}\left(x_{1}, \ldots, x_{n}\right) y_{j}
$$

para todos $x_{1} \in E_{1}, \ldots, x_{n} \in E_{n}$. Definimos $\lambda_{1}=\cdots=\lambda_{k}=1$ e, para $j>k$,

$$
\lambda_{j}=0 \in \mathbb{K}, B_{j}=0 \in \mathcal{L}\left(E_{1}, \ldots, E_{n}\right) \text { e } y_{j}=0 \in F .
$$

É óbvio que $\left(\lambda_{j}\right)_{j=1}^{\infty} \in \ell_{p^{*}}$. A igualdade (2.4) mostra que $A$ cumpre a condição (i) da definição de operador hiper- $\sigma(p)$-nuclear. De

$\sup _{\left\|x_{i}\right\| \leq 1,\left\|y^{*}\right\| \leq 1}\left(\sum_{j=1}^{\infty}\left|B_{j}\left(x_{1}, \ldots, x_{n}\right) y^{*}\left(y_{j}\right)\right|^{p}\right)^{\frac{1}{p}}=\sup _{\left\|x_{i}\right\| \leq 1,\left\|y^{*}\right\| \leq 1}\left(\sum_{j=1}^{k}\left|B_{j}\left(x_{1}, \ldots, x_{n}\right) y^{*}\left(y_{j}\right)\right|^{p}\right)^{\frac{1}{p}}<\infty$

concluímos que $A$ cumpre a condição (ii). Para cada $j>k$,

$$
\sup _{\left\|x_{i}\right\| \leq 1,\left\|y^{*}\right\| \leq 1}\left(\sum_{j=m}^{\infty}\left|B_{j}\left(x_{1}, \ldots, x_{n}\right) y^{*}\left(y_{j}\right)\right|^{p}\right)^{\frac{1}{p}}=0
$$

de onde segue trivialmente que

$$
\lim _{m \rightarrow \infty} \sup _{\left\|x_{i}\right\| \leq 1,\left\|y^{*}\right\| \leq 1}\left(\sum_{j=m}^{\infty}\left|B_{j}\left(x_{1}, \ldots, x_{n}\right) y^{*}\left(y_{j}\right)\right|^{p}\right)^{\frac{1}{p}}=0
$$

o que nos dá a condição (iii). Logo, $\sum_{j=1}^{\infty} \lambda_{j} B_{j} \otimes y_{j}$ é uma representação hiper- $\sigma(p)$-nuclear de $A$, provando que $A \in \mathcal{L}_{\mathcal{H} \sigma(p)}\left(E_{1}, \ldots, E_{n} ; F\right)$.

Precisamos tratar agora da seguinte inclusão:

$$
\mathcal{L}_{\mathcal{H} \sigma(p)}\left(E_{1}, \ldots, E_{n} ; F\right) \subseteq \overline{\mathcal{L}_{\mathcal{F}}}\left(E_{1}, \ldots, E_{n} ; F\right) .
$$


Dado $A \in \mathcal{L}_{\mathcal{H} \sigma(p)}\left(E_{1}, \ldots, E_{n} ; F\right)$, tomemos sequências $\left(\lambda_{j}\right)_{j=1}^{\infty} \in \ell_{p^{*}},\left(A_{j}\right)_{j=1}^{\infty} \subseteq \mathcal{L}\left(E_{1}, \ldots, E_{n}\right)$ e $\left(y_{j}\right)_{j=1}^{\infty} \subseteq F$ tais que

$$
A=\sum_{j=1}^{\infty} \lambda_{j} A_{j} \otimes y_{j}
$$

seja uma representação hiper- $\sigma(p)$-nuclear de $A$. Para cada $m \in \mathbb{N}$, é claro que

$$
\sum_{j=1}^{m} \lambda_{j} A_{j} \otimes y_{j} \in \mathcal{L}_{\mathcal{F}}\left(E_{1}, \ldots, E_{n} ; F\right) .
$$

Da Proposição 2.2.3 sabemos que

$$
\lim _{m \rightarrow \infty}\left\|A-\sum_{j=1}^{m} \lambda_{j} A_{j} \otimes y_{j}\right\|=0 .
$$

Isso significa que

$$
\left(\sum_{j=1}^{m} \lambda_{j} A_{j} \otimes y_{j}\right)_{m=1}^{\infty}
$$

é uma sequência de operadores de posto finito que converge para $A$ na norma $\|\cdot\|$. Logo, $A \in \overline{\mathcal{L}_{\mathcal{F}}}\left(E_{1}, \ldots, E_{n} ; F\right)$.

(b) A inclusão desejada e a desigualdade das normas seguem imediatamente das respectivas definições uma vez que todo produto de funcionais lineares contínuos é uma forma multilinear contínua.

Para a segunda afirmação considere a forma bilinear

$$
A: \ell_{2} \times \ell_{2} \longrightarrow \mathbb{K}, A\left(\left(x_{i}\right)_{i=1}^{\infty},\left(y_{i}\right)_{i=1}^{\infty}\right)=\sum_{i=1}^{\infty} x_{i} y_{i}
$$

que, pela Desigualdade de Hölder, está bem definida e é contínua. Por $A$ ter posto 1 e pelo item (a) temos

$$
A \in \mathcal{L}_{\mathcal{F}}\left({ }^{2} \ell_{2} ; \mathbb{K}\right) \subseteq \mathcal{L}_{\mathcal{H} \sigma(p)}\left({ }^{2} \ell_{2} ; \mathbb{K}\right)
$$

Vejamos que $A \notin \overline{\mathcal{L}_{f}}\left({ }^{2} \ell_{2} ; \mathbb{K}\right)$. Suponhamos, por absurdo, que $A$ seja aproximável por operadores de tipo finito. Neste caso existiria $A_{f} \in \mathcal{L}_{f}\left({ }^{2} \ell_{2} ; \mathbb{K}\right)$ tal que $\left\|A-A_{f}\right\|<\frac{1}{2}$. Por $A_{f}$ ser de tipo finito, existem $k \in \mathbb{N}$ e funcionais lineares $\varphi_{1}^{(1)}, \ldots, \varphi_{k}^{(1)}, \varphi_{1}^{(2)}, \ldots, \varphi_{k}^{(2)} \in \ell_{2}^{*}$ tais que, para todas sequências $\left(x_{i}\right)_{i=1}^{\infty},\left(y_{i}\right)_{i=1}^{\infty} \in \ell_{2}$,

$$
A_{f}\left(\left(x_{i}\right)_{i=1}^{\infty},\left(y_{i}\right)_{i=1}^{\infty}\right)=\sum_{j=1}^{k} \varphi_{j}^{(1)}\left(\left(x_{i}\right)_{i=1}^{\infty}\right) \varphi_{j}^{(2)}\left(\left(y_{i}\right)_{i=1}^{\infty}\right) .
$$


Da relação de dualidade $\ell_{2}=\ell_{2}^{*}$, para cada $j=1, \ldots, k$, existem sequências $\left(a_{i}^{(j)}\right)_{i=1}^{\infty} \mathrm{e}$ $\left(b_{i}^{(j)}\right)_{i=1}^{\infty}$ em $\ell_{2}$ tais que

$$
\varphi_{j}^{(1)}\left(\left(x_{i}\right)_{i=1}^{\infty}\right)=\sum_{i=1}^{\infty} a_{i}^{(j)} x_{i} \quad \text { e } \varphi_{j}^{(2)}\left(\left(y_{i}\right)_{i=1}^{\infty}\right)=\sum_{i=1}^{\infty} b_{i}^{(j)} y_{i} .
$$

Daí,

$$
A_{f}\left(\left(x_{i}\right)_{i=1}^{\infty},\left(y_{i}\right)_{i=1}^{\infty}\right)=\sum_{j=1}^{k}\left(\sum_{i=1}^{\infty} a_{i}^{(j)} x_{i}\right) \cdot\left(\sum_{i=1}^{\infty} b_{i}^{(j)} y_{i}\right) .
$$

Considerando os vetores canônicos unitários $\left(e_{m}\right)_{m=1}^{\infty} \subseteq \ell_{2}$, temos

$$
A_{f}\left(e_{m}, e_{m}\right)=\sum_{j=1}^{k} a_{m}^{(j)} b_{m}^{(j)}
$$

para todo $m$. Para todo $j=1, \ldots, k, \sum_{m=1}^{\infty}\left|a_{m}^{(j)}\right|^{2}$ e $\sum_{m=1}^{\infty}\left|b_{m}^{(j)}\right|^{2}$ são séries convergentes, logo seus termos gerais convergem para zero, e portanto

$$
A_{f}\left(e_{m}, e_{m}\right)=\sum_{j=1}^{k} a_{m}^{(j)} b_{m}^{(j)} \stackrel{m \rightarrow \infty}{\longrightarrow} 0 .
$$

É claro que $A\left(e_{m}, e_{m}\right)=1$ para todo $m, \operatorname{logo}$

$$
\left|A\left(e_{m}, e_{m}\right)-A_{f}\left(e_{m}, e_{m}\right)\right|=\left|1-A_{f}\left(e_{m}, e_{m}\right)\right| \stackrel{m \rightarrow \infty}{\longrightarrow} 1
$$

Existe então $m \in \mathbb{N}$ tal que $\left|A\left(e_{m}, e_{m}\right)-A_{f}\left(e_{m}, e_{m}\right)\right|>\frac{3}{4}$. Segue que

$$
\left.\frac{1}{2}>\left\|A-A_{f}\right\|=\sup _{\|x\|,\|y\| \leq 1} \mid A(x, y)\right)-A_{f}(x, y)|\geq| A\left(e_{m}, e_{m}\right)-A_{f}\left(e_{m}, e_{m}\right) \mid>\frac{3}{4} .
$$

Essa contradição prova que $A \notin \overline{\mathcal{L}_{f}}\left({ }^{2} \ell_{2} ; \mathbb{K}\right)$. Pelo Lema 2.2.4 sabemos que $\mathcal{L}_{\sigma(p)}\left({ }^{2} \ell_{2} ; \mathbb{K}\right) \subseteq$ $\overline{\mathcal{L}_{f}}\left({ }^{2} \ell_{2} ; \mathbb{K}\right)$, donde segue que $A \notin \mathcal{L}_{\sigma(p)}\left({ }^{2} \ell_{2} ; \mathbb{K}\right)$.

No resultado a seguir provaremos que a classe dos operadores multilineares hiper- $\sigma(p)$ nucleares $\mathcal{L}_{\mathcal{H} \sigma(p)}$ coincide isometricamente com o ideal de composição $\mathcal{N}_{\sigma(p)} \circ \mathcal{L}$. Isso será importante de duas formas: por um lado, decorrerá que essa nova classe de operadores multilineares é um hiper-ideal de Banach; e, por outro lado, já deixará a nova classe pronta para a representação de funcionais lineares em $\mathcal{L}_{\mathcal{H} \sigma(p)}$, assunto este que será o objeto de estudo do capítulo seguinte. 
Teorema 2.2.6. Para todo $1 \leq p<\infty$ e todos espaços de Banach $E_{1}, \ldots, E_{n}$ e $F$,

$$
\mathcal{N}_{\sigma(p)} \circ \mathcal{L}\left(E_{1}, \ldots, E_{n} ; F\right)=\mathcal{L}_{\mathcal{H} \sigma(p)}\left(E_{1}, \ldots, E_{n} ; F\right)
$$

$e\|\cdot\|_{\mathcal{N}_{\sigma(p)} \circ \mathcal{L}}=\|\cdot\|_{\mathcal{H} \sigma(p)}$.

Demonstração. Seja $A \in \mathcal{N}_{\sigma(p)} \circ \mathcal{L}\left(E_{1}, \ldots, E_{n} ; F\right)$. Existem um espaço de Banach $G$, e operadores $B \in \mathcal{L}\left(E_{1}, \ldots, E_{n} ; G\right)$ e $u \in \mathcal{N}_{\sigma(p)}(G ; F)$ tais que $A=u \circ B$.

Dado $\varepsilon>0$, como $u \in \mathcal{N}_{\sigma(p)}(G ; F)$, podemos tomar sequências $\left(\lambda_{j}\right)_{j=1}^{\infty} \in \ell_{p^{*}},\left(\varphi_{j}\right)_{j=1}^{\infty} \subseteq$ $G^{*}$ e $\left(y_{j}\right)_{j=1}^{\infty} \subseteq F$ tais que

$$
u(z)=\sum_{j=1}^{\infty} \lambda_{j} \varphi_{j}(z) y_{j}
$$

para todo $z \in G$,

$$
\begin{gathered}
\sup _{z \in B_{G}, y^{*} \in B_{F^{*}}}\left(\sum_{j=1}^{\infty}\left|\varphi_{j}(z) y^{*}\left(y_{j}\right)\right|^{p}\right)^{\frac{1}{p}}<\infty \\
\lim _{m \rightarrow \infty} \sup _{z \in B_{G}, y^{*} \in B_{F^{*}}}\left(\sum_{j=m}^{\infty}\left|\varphi_{j}(z) y^{*}\left(y_{j}\right)\right|^{p}\right)^{\frac{1}{p}}=0 \text { e } \\
\left\|\left(\lambda_{j}\right)_{j=1}^{\infty}\right\|_{p^{*}} \cdot \sup _{z \in B_{G}, y^{*} \in B_{F^{*}}}\left(\sum_{j=1}^{\infty}\left|\varphi_{j}(z) y^{*}\left(y_{j}\right)\right|^{p}\right)^{\frac{1}{p}} \leq(1+\varepsilon)\|u\|_{\mathcal{N}_{\sigma(p)}} .
\end{gathered}
$$

De $A=u \circ B$ segue que

$$
\begin{aligned}
A\left(x_{1}, \ldots, x_{n}\right) & =(u \circ B)\left(x_{1}, \ldots, x_{n}\right)=u\left(B\left(x_{1}, \ldots, x_{n}\right)\right) \\
& =\sum_{j=1}^{\infty} \lambda_{j} \varphi_{j}\left(B\left(x_{1}, \ldots, x_{n}\right)\right) y_{j} \\
& =\sum_{j=1}^{\infty} \lambda_{j}\left(\varphi_{j} \circ B\right) \otimes y_{j}\left(x_{1}, \ldots, x_{n}\right)
\end{aligned}
$$

para todos $x_{1} \in E_{1}, \ldots, x_{n} \in E_{n}$. Vejamos que $A=\sum_{j=1}^{\infty} \lambda_{j}\left(\varphi_{j} \circ B\right) \otimes y_{j}$ é uma representação hiper- $\sigma(p)$-nuclear de $A$. Com efeito, já sabemos que $\left(\lambda_{j}\right)_{j=1}^{\infty} \in \ell_{p^{*}},\left(\varphi_{j} \circ B\right)_{j=1}^{\infty} \subseteq$ $\mathcal{L}\left(E_{1}, \ldots, E_{n}\right)$ e $\left(y_{j}\right)_{j=1}^{\infty} \subseteq F$. Temos também

$\sup _{x_{i} \in B_{E_{i}}, y^{*} \in B_{F^{*}}}\left(\sum_{j=1}^{\infty}\left|\left(\varphi_{j} \circ B\right)\left(x_{1}, \ldots, x_{n}\right) y^{*}\left(y_{j}\right)\right|^{p}\right)^{\frac{1}{p}}$ 


$$
\begin{aligned}
& =\sup _{x_{i} \in B_{E_{i}}, y^{*} \in B_{F^{*}}}\left(\sum_{j=1}^{\infty} \mid\left(\left.\varphi_{j}\left(B\left(x_{1}, \ldots, x_{n}\right)\right) y^{*}\left(y_{j}\right)\right|^{p}\right)^{\frac{1}{p}}\right. \\
& (B \neq 0)=\|B\| \cdot \sup _{x_{i} \in B_{E_{i}}, y^{*} \in B_{F^{*}}}\left(\sum_{j=1}^{\infty}\left|\varphi_{j}\left[\left(\frac{B}{\|B\|}\right)\left(x_{1}, \ldots, x_{n}\right)\right] y^{*}\left(y_{j}\right)\right|^{p}\right)^{\frac{1}{p}} \\
& \leq\|B\| \cdot \sup _{z \in B_{G}, y^{*} \in B_{F^{*}}}\left(\sum_{j=1}^{\infty}\left|\varphi_{j}(z) y^{*}\left(y_{j}\right)\right|^{p}\right)^{\frac{1}{p}}<\infty
\end{aligned}
$$

e, repetindo o argumento,

$$
\begin{aligned}
\lim _{m \rightarrow \infty} \sup _{x_{i} \in B_{E_{i}}, y^{*} \in B_{F}}( & \left(\sum_{j=m}^{\infty}\left|\left(\varphi_{j} \circ B\right)\left(x_{1}, \ldots, x_{n}\right) y^{*}\left(y_{j}\right)\right|^{p}\right)^{\frac{1}{p}} \\
& \leq \lim _{m \rightarrow \infty}\|B\| \cdot \sup _{z \in B_{G}, y^{*} \in B_{F^{*}}}\left(\sum_{j=m}^{\infty}\left|\varphi_{j}(z) y^{*}\left(y_{j}\right)\right|^{p}\right)^{\frac{1}{p}} \\
& =\|B\| \cdot \lim _{m \rightarrow \infty} \sup _{z \in B_{G}, y^{*} \in B_{F^{*}}}\left(\sum_{j=m}^{\infty}\left|\varphi_{j}(z) y^{*}\left(y_{j}\right)\right|^{p}\right)^{\frac{1}{p}}=0 .
\end{aligned}
$$

Portanto $A=\sum_{j=1}^{\infty} \lambda_{j}\left(\varphi_{j} \circ B\right) \otimes y_{j} \in \mathcal{L}_{\mathcal{H} \sigma(p)}\left(E_{1}, \ldots, E_{n} ; F\right) \mathrm{e}$

$$
\begin{aligned}
\|A\|_{\mathcal{H} \sigma(p)} & \leq\left\|\left(\lambda_{j}\right)_{j=1}^{\infty}\right\|_{p^{*}} \cdot \sup _{x_{i} \in B_{E_{i}}, y^{*} \in B_{F^{*}}}\left(\sum_{j=1}^{\infty}\left|\left(\varphi_{j} \circ B\right)\left(x_{1}, \ldots, x_{n}\right) y^{*}\left(y_{j}\right)\right|^{p}\right)^{\frac{1}{p}} \\
& (B \neq 0)=\|B\| \cdot\left\|\left(\lambda_{j}\right)_{j=1}^{\infty}\right\|_{p^{*}} \cdot \sup _{x_{i} \in B_{E_{i}}, y^{*} \in B_{F^{*}}}\left(\sum_{j=1}^{\infty}\left|\varphi_{j}\left[\left(\frac{B}{\|B\|}\right)\left(x_{1}, \ldots, x_{n}\right)\right] y^{*}\left(y_{j}\right)\right|^{p}\right)^{\frac{1}{p}} \\
& \leq\|B\| \cdot\left\|\left(\lambda_{j}\right)_{j=1}^{\infty}\right\|_{p^{*}} \cdot \sup _{z \in B_{G}, y^{*} \in B_{F^{*}}}\left(\sum_{j=1}^{\infty}\left|\varphi_{j}(z) y^{*}\left(y_{j}\right)\right|^{p}\right)^{\frac{1}{p}} \\
& \leq(1+\varepsilon)\|B\| \cdot\|u\|_{\mathcal{N}_{\sigma(p)} .}
\end{aligned}
$$

Fazendo $\varepsilon \longrightarrow 0^{+}$, obtemos $\|A\|_{\mathcal{H} \sigma(p)} \leq\|B\| \cdot\|u\|_{\mathcal{N}_{\sigma(p)}}$ para toda fatoração $A=u \circ B$, com $u \in \mathcal{N}_{\sigma(p)}(G ; F)$. Tomando o ínfimo sobre todas essas fatorações concluímos que $\|A\|_{\mathcal{H} \sigma(p)} \leq\|A\|_{\mathcal{N}_{\sigma(p)} \circ \mathcal{L}}$.

Reciprocamente, dados $A \in \mathcal{L}_{\mathcal{H} \sigma(p)}\left(E_{1}, \ldots, E_{n} ; F\right)$ e $\varepsilon>0$, existem sequências $\left(\lambda_{j}\right)_{j=1}^{\infty} \in$ $\ell_{p^{*}},\left(B_{j}\right)_{j=1}^{\infty} \subseteq \mathcal{L}\left(E_{1}, \ldots, E_{n}\right)$ e $\left(y_{j}\right)_{j=1}^{\infty} \subseteq F$ tais que

$$
\sup _{x_{i} \in B_{E_{i}}, y^{*} \in B_{F^{*}}}\left(\sum_{j=1}^{\infty}\left|B_{j}\left(x_{1}, \ldots, x_{n}\right) y^{*}\left(y_{j}\right)\right|^{p}\right)^{\frac{1}{p}}<\infty
$$




$$
\begin{gathered}
\lim _{m \rightarrow \infty} \sup _{x_{i} \in B_{E_{i}}, y^{*} \in B_{F^{*}}}\left(\sum_{j=m}^{\infty}\left|B_{j}\left(x_{1}, \ldots, x_{n}\right) y^{*}\left(y_{j}\right)\right|^{p}\right)^{\frac{1}{p}}=0 \mathrm{e} \\
\left\|\left(\lambda_{j}\right)_{j=1}^{\infty}\right\|_{p^{*}} \cdot \sup _{x_{i} \in B_{E_{i}}, y^{*} \in B_{F^{*}}}\left(\sum_{j=1}^{\infty}\left|B_{j}\left(x_{1}, \ldots, x_{n}\right) y^{*}\left(y_{j}\right)\right|^{p}\right)^{\frac{1}{p}} \leq(1+\varepsilon)\|A\|_{\mathcal{H} \sigma(p)} .
\end{gathered}
$$

Pela Proposição 2.2.3 sabemos que a convergência $A=\sum_{j=1}^{\infty} \lambda_{j} B_{j} \otimes y_{j}$ se dá na norma usual de $\mathcal{L}\left(E_{1}, \ldots, E_{n} ; F\right)$. Usando que a correspondência

$$
B \in \mathcal{L}\left(E_{1}, \ldots, E_{n} ; F\right) \mapsto B_{L} \in \mathcal{L}\left(E_{1} \widehat{\otimes}_{\pi} \cdots \widehat{\otimes}_{\pi} E_{n} ; F\right)
$$

é um isomorfismo, logo linear e contínua, do Lema 1.2.15 segue que

$$
\begin{aligned}
A_{L} & =\left(\sum_{j=1}^{\infty} \lambda_{j} B_{j} \otimes y_{j}\right)_{L}=\left(\lim _{m} \sum_{j=1}^{m} \lambda_{j} B_{j} \otimes y_{j}\right)_{L}=\lim _{m}\left(\sum_{j=1}^{m} \lambda_{j} B_{j} \otimes y_{j}\right)_{L} \\
& =\lim _{m} \sum_{j=1}^{m} \lambda_{j}\left(B_{j} \otimes y_{j}\right)_{L}=\lim _{m} \sum_{j=1}^{m} \lambda_{j}\left(B_{j}\right)_{L} \otimes y_{j}=\sum_{j=1}^{\infty} \lambda_{j}\left(B_{j}\right)_{L} \otimes y_{j},
\end{aligned}
$$

na norma de $\mathcal{L}\left(E_{1} \widehat{\otimes}_{\pi} \cdots \widehat{\otimes}_{\pi} E_{n} ; F\right)$. Provemos que $\sum_{j=1}^{\infty} \lambda_{j}\left(B_{j}\right)_{L} \otimes y_{j}$ é uma representação hiper- $\sigma(p)$-nuclear de $A_{L}$.

Fixe $m \in \mathbb{N}$. Dados $k \geq m$, como a norma de um operador multilinear coincide com a norma da sua linearização, temos

$$
\left\|\sum_{j=m}^{k} B_{j} \otimes y_{j}\right\|=\left\|\left(\sum_{j=m}^{k} B_{j} \otimes y_{j}\right)_{L}\right\|=\left\|\sum_{j=m}^{k}\left(B_{j}\right)_{L} \otimes y_{j}\right\| .
$$

Para todos escalares $\lambda_{1}, \ldots, \lambda_{m}$, segue da dualidade $\left(\ell_{p}^{m}\right)^{*}=\ell_{p^{*}}^{m}$ e do Teorema de Hahn-Banach que existem escalares $\theta_{1}, \ldots, \theta_{m}$ tais que

$$
\left\|\left(\theta_{j}\right)_{j=1}^{m}\right\|_{p^{*}}=1 \text { e }\left(\sum_{j=1}^{m}\left|\lambda_{j}\right|^{p}\right)^{1 / p}=\left|\sum_{j=1}^{m} \theta_{j} \lambda_{j}\right| .
$$

Assim, para cada $z \in B_{E_{1} \widehat{\otimes}_{\pi} \cdots \widehat{\otimes}_{\pi} E_{n}}$, cada $y^{*} \in B_{F^{*}}$ e cada $j=m, \ldots, k$, existem escalares $\theta_{m}, \ldots, \theta_{k}$ tais que

$$
\left\|\left(\theta_{j}\right)_{j=m}^{k}\right\|_{p^{*}}=1 \text { e }\left(\sum_{j=m}^{k}\left|\left(B_{j}\right)_{L}(z) y^{*}\left(y_{j}\right)\right|^{p}\right)^{1 / p}=\left|\sum_{j=m}^{k} \theta_{j}\left(B_{j}\right)_{L}(z) y^{*}\left(y_{j}\right)\right| \text {. }
$$


Nas contas a seguir, usaremos os seguintes instrumentos na seguinte ordem: (i) as igualdades em (2.9), (ii) a igualdade (2.8), (iii) o Teorema de Hahn-Banach na forma $\|x\|=\sup _{\|\varphi\| \leq 1}|\varphi(x)|$, (iv) a Desigualdade de Hölder para $\frac{1}{p}+\frac{1}{p^{*}}=1$.

$$
\begin{aligned}
& \left(\sum_{j=m}^{k}\left|\left(B_{j}\right)_{L}(z) y^{*}\left(y_{j}\right)\right|^{p}\right)^{\frac{1}{p}}=\left|\sum_{j=m}^{k} \theta_{j}\left(B_{j}\right)_{L}(z) y^{*}\left(y_{j}\right)\right|=\left|y^{*}\left(\sum_{j=m}^{k} \theta_{j}\left(B_{j}\right)_{L}(z) y_{j}\right)\right| \\
& \leq\left\|y^{*}\right\| \cdot\left\|\sum_{j=m}^{k} \theta_{j}\left(B_{j}\right)_{L}(z) y_{j}\right\| \leq\left\|\sum_{j=m}^{k} \theta_{j}\left(B_{j}\right)_{L}(z) y_{j}\right\| \\
& =\left\|\left(\sum_{j=m}^{k}\left(B_{j}\right)_{L} \otimes\left(\theta_{j} y_{j}\right)\right)(z)\right\| \leq\left\|\sum_{j=m}^{k}\left(B_{j}\right)_{L} \otimes\left(\theta_{j} y_{j}\right)\right\| \cdot\|z\| \\
& \leq\left\|\sum_{j=m}^{k}\left(B_{j}\right)_{L} \otimes\left(\theta_{j} y_{j}\right)\right\|=\left\|\sum_{j=m}^{k} \theta_{j}\left(B_{j}\right)_{L} \otimes y_{j}\right\|=\left\|\sum_{j=m}^{k} \theta_{j} B_{j} \otimes y_{j}\right\| \\
& =\sup _{\left\|\widetilde{x_{i}}\right\| \leq 1}\left\|\left(\sum_{j=m}^{k} \theta_{j} B_{j} \otimes y_{j}\right)\left(\widetilde{x_{1}}, \ldots, \widetilde{x_{n}}\right)\right\| \\
& =\sup _{\left\|\widetilde{x_{i}}\right\| \leq 1}\left\|\sum_{j=m}^{k} \theta_{j} B_{j}\left(\widetilde{x_{1}}, \ldots, \widetilde{x_{n}}\right) y_{j}\right\| \\
& =\sup _{\left\|\widetilde{x_{i}}\right\| \leq 1} \sup _{\left\|\widetilde{y^{*}}\right\| \leq 1}\left|\widetilde{y^{*}}\left(\sum_{j=m}^{k} \theta_{j} B_{j}\left(\widetilde{x_{1}}, \ldots, \widetilde{x_{n}}\right) y_{j}\right)\right| \\
& =\sup _{\left\|\widetilde{x_{i}}\right\| \leq 1,\left\|\widetilde{y^{*}}\right\| \leq 1}\left|\sum_{j=m}^{k} \theta_{j} B_{j}\left(\widetilde{x_{1}}, \ldots, \widetilde{x_{n}}\right) \widetilde{y^{*}}\left(y_{j}\right)\right| \\
& \leq \sup _{\left\|\widetilde{x_{i}}\right\| \leq 1,\left\|\widetilde{y^{*}}\right\| \leq 1} \sum_{j=m}^{k}\left|\theta_{j} B_{j}\left(\widetilde{x_{1}}, \ldots, \widetilde{x_{n}}\right) \widetilde{y^{*}}\left(y_{j}\right)\right| \\
& \leq \sup _{\left\|\widetilde{x_{i}}\right\| \leq 1,\left\|\widetilde{y^{*}}\right\| \leq 1}\left(\sum_{j=m}^{k}\left|\theta_{j}\right|^{p^{*}}\right)^{\frac{1}{p^{*}}} \cdot\left(\sum_{j=m}^{k}\left|B_{j}\left(\widetilde{x_{1}}, \ldots, \widetilde{x_{n}}\right) \widetilde{y^{*}}\left(y_{j}\right)\right|^{p}\right)^{\frac{1}{p}} \\
& =\sup _{\left\|\widetilde{x_{i}}\right\| \leq 1,\left\|\widetilde{y^{*}}\right\| \leq 1}\left(\sum_{j=m}^{k}\left|B_{j}\left(\widetilde{x_{1}}, \ldots, \widetilde{x_{n}}\right) \widetilde{y^{*}}\left(y_{j}\right)\right|^{p}\right)^{\frac{1}{p}} \text {. }
\end{aligned}
$$

Assim, tomando o supremo sobre $\|z\|_{\pi} \leq 1$ e $\left\|y^{*}\right\| \leq 1$, temos

$$
\sup _{\|z\|\left\|_{\pi} \leq 1,\right\| y^{*} \| \leq 1}\left(\sum_{j=m}^{k}\left|\left(B_{j}\right)_{L}(z) y^{*}\left(y_{j}\right)\right|^{p}\right)^{\frac{1}{p}} \leq \sup _{\left\|x_{i}\right\| \leq 1,\left\|y^{*}\right\| \leq 1}\left(\sum_{j=m}^{k}\left|B_{j}\left(x_{1}, \ldots, x_{n}\right) y^{*}\left(y_{j}\right)\right|^{p}\right)^{\frac{1}{p}}
$$


para todos $k \geq m$. Usando (2.10) e (2.5),

$$
\begin{aligned}
\sup _{\|z\|_{\pi} \leq 1,\left\|y^{*}\right\| \leq 1}\left(\sum_{j=1}^{\infty}\left|\left(B_{j}\right)_{L}(z) y^{*}\left(y_{j}\right)\right|^{p}\right)^{\frac{1}{p}} & =\sup _{\|z\|_{\pi} \leq 1,\left\|y^{*}\right\| \leq 1} \sup _{k}\left(\sum_{j=1}^{k}\left|\left(B_{j}\right)_{L}(z) y^{*}\left(y_{j}\right)\right|^{p}\right)^{\frac{1}{p}} \\
& =\sup _{k} \sup _{\|z\| n \leq 1,\left\|y^{*}\right\| \leq 1}\left(\sum_{j=1}^{k}\left|\left(B_{j}\right)_{L}(z) y^{*}\left(y_{j}\right)\right|^{p}\right)^{\frac{1}{p}} \\
& \leq \sup _{k} \sup _{\left\|x_{i}\right\| \leq 1,\left\|y^{*}\right\| \leq 1}\left(\sum_{j=1}^{k}\left|B_{j}\left(x_{1}, \ldots, x_{n}\right) y^{*}\left(y_{j}\right)\right|^{p}\right)^{\frac{1}{p}} \\
& =\sup _{\left\|x_{i}\right\| \leq 1,\left\|y^{*}\right\| \leq 1} \sup _{k}\left(\sum_{j=1}^{k}\left|B_{j}\left(x_{1}, \ldots, x_{n}\right) y^{*}\left(y_{j}\right)\right|^{p}\right)^{\frac{1}{p}} \\
& =\sup _{\left\|x_{i}\right\| \leq 1,\left\|y^{*}\right\| \leq 1}\left(\sum_{j=1}^{\infty}\left|B_{j}\left(x_{1}, \ldots, x_{n}\right) y^{*}\left(y_{j}\right)\right|^{p}\right)^{\frac{1}{p}}<\infty .
\end{aligned}
$$

E usando (2.10) e (2.6),

$$
\begin{aligned}
\sup _{\|z\|_{\pi} \leq 1,\left\|y^{*}\right\| \leq 1} & \left(\sum_{j=m}^{\infty}\left|\left(B_{j}\right)_{L}(z) y^{*}\left(y_{j}\right)\right|^{p}\right)^{\frac{1}{p}}=\sup _{\|z\|_{\pi} \leq 1,\left\|y^{*}\right\| \leq 1} \sup _{k \geq m}\left(\sum_{j=m}^{k}\left|\left(B_{j}\right)_{L}(z) y^{*}\left(y_{j}\right)\right|^{p}\right)^{\frac{1}{p}} \\
& =\sup _{k \geq m} \sup _{\|z\|}\left(\sum_{\pi \leq 1,\left\|y^{*}\right\| \leq 1}^{k}\left|\left(B_{j}\right)_{L}(z) y^{*}\left(y_{j}\right)\right|^{p}\right)^{\frac{1}{p}} \\
& \leq \sup _{k \geq m} \sup _{\left\|x_{i}\right\| \leq 1,\left\|y^{*}\right\| \leq 1}\left(\sum_{j=m}^{k}\left|B_{j}\left(x_{1}, \ldots, x_{n}\right) y^{*}\left(y_{j}\right)\right|^{p}\right)^{\frac{1}{p}} \\
& =\sup _{\left\|x_{i}\right\| \leq 1,\left\|y^{*}\right\| \leq 1} \sup _{k \geq m}\left(\sum_{j=m}^{k}\left|B_{j}\left(x_{1}, \ldots, x_{n}\right) y^{*}\left(y_{j}\right)\right|^{p}\right)^{\frac{1}{p}} \\
& =\sup _{\left\|x_{i}\right\| \leq 1,\left\|y^{*}\right\| \leq 1}\left(\sum_{j=m}^{\infty}\left|B_{j}\left(x_{1}, \ldots, x_{n}\right) y^{*}\left(y_{j}\right)\right|^{p}\right)^{\frac{1}{p}} \stackrel{m \rightarrow \infty}{\longrightarrow} 0 .
\end{aligned}
$$

Isso prova que $\sum_{j=1}^{\infty} \lambda_{j}\left(B_{j}\right)_{L} \otimes y_{j}$ é uma representação $\sigma(p)$-nuclear de $A_{L}$, isto é, $A_{L}$ é um operador linear $\sigma(p)$-nuclear. Segue da Proposição 1.4.8 que $A \in \mathcal{N}_{\sigma(p)} \circ \mathcal{L}\left(E_{1}, \ldots, E_{n} ; F\right)$. E, aplicando (2.10) uma vez mais e também (2.7), segue também que

$$
\|A\|_{\mathcal{N}_{\sigma(p)} \circ \mathcal{L}}=\left\|A_{L}\right\|_{\mathcal{N}_{\sigma(p)}} \leq\left\|\left(\lambda_{j}\right)_{j=1}^{\infty}\right\|_{p^{*}} \cdot \sup _{\|z\|_{\pi} \leq 1, y^{*} \in B_{F^{*}}}\left(\sum_{j=1}^{\infty}\left|\left(B_{L}\right)_{j}(z) y^{*}\left(y_{j}\right)\right|^{p}\right)^{\frac{1}{p}}
$$




$$
\begin{aligned}
& =\left\|\left(\lambda_{j}\right)_{j=1}^{\infty}\right\|_{p^{*}} \cdot \sup _{k} \sup _{\|z\|_{\pi} \leq 1, y^{*} \in B_{F^{*}}}\left(\sum_{j=1}^{k}\left|\left(B_{L}\right)_{j}(z) y^{*}\left(y_{j}\right)\right|^{p}\right)^{\frac{1}{p}} \\
& \leq\left\|\left(\lambda_{j}\right)_{j=1}^{\infty}\right\|_{p^{*}} \cdot \sup _{k} \sup _{x_{i} \in B_{E_{i}}, y^{*} \in B_{F^{*}}}\left(\sum_{j=1}^{k}\left|B_{j}\left(x_{1}, \ldots, x_{n}\right) y^{*}\left(y_{j}\right)\right|^{p}\right)^{\frac{1}{p}} \\
& =\left\|\left(\lambda_{j}\right)_{j=1}^{\infty}\right\|_{p^{*}} \cdot \sup _{x_{i} \in B_{E_{i}}, y^{*} \in B_{F^{*}}}\left(\sum_{j=1}^{\infty}\left|B_{j}\left(x_{1}, \ldots, x_{n}\right) y^{*}\left(y_{j}\right)\right|^{p}\right)^{\frac{1}{p}} \\
& \leq(1+\varepsilon)\|A\|_{\mathcal{H} \sigma(p)},
\end{aligned}
$$

para todo $\varepsilon>0$, onde a primeira igualdade segue da Proposição 1.4.9. Fazendo $\varepsilon \longrightarrow 0^{+}$, concluímos que $\|A\|_{\mathcal{N}_{\sigma(p)} \circ \mathcal{L}} \leq\|A\|_{\mathcal{H} \sigma(p)}$.

Terminamos o capítulo com o corolário a seguir, mas chamamos a atenção do leitor para o fato de que, no próximo capítulo, $\mathcal{L}_{\mathcal{H} \sigma(p)}$ ser um ideal de composição será mais importante do que ser simplesmente um hiper-ideal de Banach.

Corolário 2.2.7. A classe $\left(\mathcal{L}_{\mathcal{H} \sigma(p)},\|\cdot\|_{\mathcal{H} \sigma(p)}\right)$ dos operadores multilineares hiper- $\sigma(p)$ nucleares é um hiper-ideal de Banach.

Demonstração. Pelo Teorema 2.2.6, temos que $\mathcal{L}_{\mathcal{H} \sigma(p)}$ é um ideal de composição, e pelo Teorema 1.5.2 concluímos que $\mathcal{L}_{\mathcal{H} \sigma(p)}$ é um hiper-ideal de Banach. 


\section{Capítulo 3}

\section{Representação de Duais}

A transformada de Borel é uma técnica poderosa para representar duais de espaços de operadores lineares e multilineares, veja, por exemplo, [4, 31, 37, 46, 47, 52]. Para operadores multilineares a técnica foi introduzida por Gupta [46, 47]. Vejamos uma breve descrição dessa técnica.

Dado um subespaço $\mathcal{M}\left(E_{1}, \ldots, E_{n} ; F\right)$ do espaço $\mathcal{L}\left(E_{1}, \ldots, E_{n} ; F\right)$ dos operadores $n$ lineares contínuos munido de uma norma completa $\|\cdot\|_{\mathcal{M}}$ e contendo os operadores de tipo finito, o operador linear e contínuo

$$
\begin{gathered}
\beta_{n}:\left(\mathcal{M}\left(E_{1}, \ldots, E_{n} ; F\right),\|\cdot\|_{\mathcal{M}}\right)^{*} \longrightarrow \mathcal{L}\left(E_{1}^{*}, \ldots, E_{n}^{*} ; F^{*}\right) \\
\beta_{n}(\phi)\left(x_{1}^{*}, \ldots, x_{n}^{*}\right)(y)=\phi\left(x_{1}^{*} \otimes \cdots \otimes x_{n}^{*} \otimes y\right),
\end{gathered}
$$

é chamado de transformada de Borel. Note que o fato do subespaço conter os operadores de tipo finito é essencial para a boa definição de $\beta_{n}$.

A técnica consiste em identificar um subespaço vetorial $\mathcal{R}\left(E_{1}^{*}, \ldots, E_{n}^{*} ; F^{*}\right)$ do espaço $\mathcal{L}\left(E_{1}^{*}, \ldots, E_{n}^{*} ; F^{*}\right)$ e muní-lo de uma norma completa $\|\cdot\|_{\mathcal{R}}$ de maneira que a transformada de Borel multilinear

$$
\begin{gathered}
\beta_{n}:\left(\mathcal{M}\left(E_{1}, \ldots, E_{n} ; F\right),\|\cdot\|_{\mathcal{M}}\right)^{*} \longrightarrow\left(\mathcal{R}\left(E_{1}^{*}, \ldots, E_{n}^{*} ; F^{*}\right),\|\cdot\|_{\mathcal{R}}\right) \\
\beta_{n}(\phi)\left(x_{1}^{*}, \ldots, x_{n}^{*}\right)(y)=\phi\left(x_{1}^{*} \otimes \cdots \otimes x_{n}^{*} \otimes y\right),
\end{gathered}
$$

seja um isomorfismo isométrico.

Isso foi feito muitas vezes na literatura, e muitas aplicações dessa técnica foram obtidas, incluindo aplicações na área de dinâmica linear (veja $[15,25,35,36,38,39,40,41,58]$ ). Destacaremos três exemplos de aplicações da transformada de Borel que nos serão úteis mais adiante. 
Exemplo 3.0.1. Uma adaptação do caso polinomial feito em [31] mostra que, para quaisquer espaços de Banach $E_{1}, \ldots, E_{n}$ e $F$ tais que $E_{1}^{*}, \ldots, E_{n}^{*}$ possuam a propriedade da aproximação, então os espaços

$$
\left[\mathcal{L}_{\mathcal{N}}\left(E_{1}, \ldots, E_{n} ; F\right)\right]^{*} \text { e } \mathcal{L}\left(E_{1}^{*}, \ldots, E_{n}^{*} ; F^{*}\right)
$$

são isomorfos isometricamente por meio da transformada de Borel.

Exemplo 3.0.2. [52, Theorem 4.1] Se $E_{1}^{*}, \ldots, E_{n}^{*}$ têm a propriedade da aproximação limitada, então, para todo espaço de Banach $F$, os espaços

$$
\left[\mathcal{L}_{\mathcal{N}}^{\left(s ; r_{1}, \ldots, r_{n}\right)}\left(E_{1}, \ldots, E_{n} ; F\right)\right]^{*} \text { e } \mathcal{L}_{a s}^{\left(s^{*} ; r_{1}^{*}, \ldots, r_{n}^{*}\right)}\left(E_{1}^{*}, \ldots, E_{n}^{*} ; F^{*}\right)
$$

são isomorfos isometricamente por meio da transformada de Borel.

Para o terceiro exemplo precisamos definir a seguinte classe de operadores multilineares.

Definição 3.0.3. [15, Definition 3.1] Seja $1 \leq q \leq p$. Um operador $n$-linear $A \in$ $\mathcal{L}\left(E_{1}, \ldots, E_{n} ; F^{*}\right)$ é quase- $\tau(p ; q)$-somante se existe uma constante $C \geq 0$ tal que

$$
\left(\sum_{j=1}^{m}\left|A\left(x_{1 j}, \ldots, x_{n j}\right)\left(y_{j}\right)\right|^{p}\right)^{\frac{1}{p}} \leq C \sup _{x_{i}^{*} \in B_{E_{i}^{*}, y^{*} \in B_{F^{*}}}}\left(\sum_{j=1}^{m}\left|x_{1}^{*}\left(x_{1 j}\right) \cdots x_{n}^{*}\left(x_{n j}\right) y^{*}\left(y_{j}\right)\right|^{q}\right)^{\frac{1}{q}},
$$

para todos $m \in \mathbb{N}, x_{i j} \in E_{i}$ e $y_{j} \in F, i=1, \ldots, n, j=1, \ldots, m$. O ínfimo de todas as constantes $C$ é denotado por $\|A\|_{q \tau(p ; q)}$. Já o espaço de todos os operadores $n$-lineares quase- $\tau(p ; q)$-somantes será denotado por $\mathcal{L}_{q \tau(p ; q)}\left(E_{1}, \ldots, E_{n} ; F^{*}\right)$.

No caso $n=1$ temos o espaço dos operadores lineares quase- $\tau(p ; q)$-somantes, que será denotado por $\mathcal{L}_{q \tau(p ; q)}\left(E ; F^{*}\right)$. Quando $p=q$ dizemos que o operador é quase- $\tau(p)$-somante e denotamos o espaço correspondente simplesmente por $\mathcal{L}_{q \tau(p)}\left(E ; F^{*}\right)$.

Cálculos rotineiros mostram que $\left(\mathcal{L}_{q \tau(p ; q)}\left(E ; F^{*}\right),\|\cdot\|_{q \tau(p ; q)}\right)$ é um espaço de Banach.

É claro que a classe dos operadores lineares quase- $\tau(p ; q)$-somantes não é um ideal de operadores, pois está definida apenas para operadores a valores em espaços duais. Sobre a possibilidade de estender essa classe a ideal de operadores, veja [16].

Exemplo 3.0.4. [15, Theorem 3.4] Se $E_{1}^{*}, \ldots, E_{n}^{*}$ têm a propriedade da aproximação limitada, então os espaços

$$
\left[\mathcal{L}_{\sigma(p)}\left(E_{1}, \ldots, E_{n} ; F\right)\right]^{*} \text { e } \mathcal{L}_{q \tau(p)}\left(E_{1}^{*}, \ldots, E_{n}^{*} ; F^{*}\right)
$$


são isometricamente isomorfos via transformada de Borel, para qualquer espaço de Banach $F$. Em particular, se $E^{*}$ tem a propriedade da aproximação limitada, então $\left[\mathcal{N}_{\sigma(p)}(E ; F)\right]^{*}$ e $\mathcal{L}_{q \tau(p)}\left(E^{*} ; F^{*}\right)$ são isometricamente isomorfos por meio da transformada de Borel linear.

O objetivo deste capítulo é introduzir uma variante da transformada de Borel multilinear, por nós chamada de hiper-transformada de Borel, que permitirá a representação de duais de espaços de operadores multilineares que não são alcançados pela técnica da transformada de Borel.

No caso linear $n=1$, a transformada de Borel

$$
\beta_{1}:\left(\mathcal{M}(E ; F),\|\cdot\|_{\mathcal{M}}\right)^{*} \longrightarrow \mathcal{L}\left(E^{*} ; F^{*}\right), \beta_{1}(\phi)\left(x^{*}\right)(y)=\phi\left(x^{*} \otimes y\right),
$$

será chamada de transformada de Borel linear e denotada por $\beta$.

\subsection{A hiper-transformada de Borel}

Uma restrição forte da técnica da transformada de Borel é que uma condição necessária para que a transformada de Borel $\beta_{n}$ seja um isomorfismo isométrico é que os operadores de tipo finito sejam $\|\cdot\|_{\mathcal{M}}$-densos em $\mathcal{M}\left(E_{1}, \ldots, E_{n} ; F\right)$. Mesmo com essa restrição, muitos resultados e aplicações foram obtidos. Entretanto, muitas classes importantes de operadores multilineares, por exemplo os operadores multilineares compactos, não satisfazem essa condição.

Nesta seção introduziremos a hiper-transformada de Borel, que se prestará à representação de duais de espaços de operadores multilineares que não são alcançados pela transformada de Borel por não se verificar a densidade dos operadores de tipo finito. Aplicações desta técnica, inclusive para os operadores multilineares compactos, serão dadas na seção seguinte.

Proposição 3.1.1. Seja $\mathcal{H}\left(E_{1}, \ldots, E_{n} ; F\right)$ um subespaço vetorial de $\mathcal{L}\left(E_{1}, \ldots, E_{n} ; F\right)$ munido de uma norma completa $\|\cdot\|_{\mathcal{H}}$ contendo os operadores de posto finito e tal que $\|A \otimes y\|_{\mathcal{H}} \leq\|A\| \cdot\|y\|$ para todos $A \in \mathcal{L}\left(E_{1}, \ldots, E_{n}\right)$ e $y \in F$. Então:

(a) A aplicação

$$
\mathcal{B}_{n}:\left(\mathcal{H}\left(E_{1}, \ldots, E_{n} ; F\right),\|\cdot\|_{\mathcal{H}}\right)^{*} \longrightarrow \mathcal{L}\left(\mathcal{L}\left(E_{1}, \ldots, E_{n}\right) ; F^{*}\right), \mathcal{B}_{n}(\phi)(A)(y)=\phi(A \otimes y),
$$

é um operador linear bem definido, contínuo com $\left\|\mathcal{B}_{n}\right\| \leq 1$. 
(b) $\mathcal{B}_{n}$ é injetor se, e somente se, o subespaço dos operadores de posto finito é $\|\cdot\|_{\mathcal{H}}$-denso em $\mathcal{H}\left(E_{1}, \ldots, E_{n} ; F\right)$.

Demonstração. (a) Vejamos que o operador $\mathcal{B}_{n}$ está bem definido. Para $A \in \mathcal{L}\left(E_{1}, \ldots, E_{n}\right)$ e $y \in F$,

$$
A \otimes y \in \mathcal{L}_{\mathcal{F}}\left(E_{1}, \ldots, E_{n} ; F\right) \subseteq \mathcal{H}\left(E_{1}, \ldots, E_{n} ; F\right),
$$

e portanto podemos calcular $\mathcal{B}_{n}(\phi)(A)(y)$ para todo funcional $\phi \in\left[\mathcal{H}\left(E_{1}, \ldots, E_{n} ; F\right)\right]^{*}$.

A linearidade de $\mathcal{B}_{n}(\phi)(A)$ é simples de ser checada, e sua continuidade segue de

$$
\left|\mathcal{B}_{n}(\phi)(A)(y)\right|=|\phi(A \otimes y)| \leq\|\phi\|_{\mathcal{H}^{*}} \cdot\|A \otimes y\|_{\mathcal{H}} \leq\|\phi\|_{\mathcal{H}^{*}} \cdot\|A\| \cdot\|y\|,
$$

para todo $y \in F$. Isso prova que $\mathcal{B}_{n}(\phi)(A) \in F^{*}$. A linearidade de $\mathcal{B}_{n}(\phi): \mathcal{L}\left(E_{1}, \ldots, E_{n}\right) \longrightarrow$ $F^{*}$ é imediata e sua continuidade segue de

$$
\begin{aligned}
\left\|\mathcal{B}_{n}(\phi)(A)\right\| & =\sup _{y \in B_{F}}\left|\mathcal{B}_{n}(\phi)(A)(y)\right|=\sup _{y \in B_{F}}|\phi(A \otimes y)| \\
& \leq \sup _{y \in B_{F}}\|\phi\|_{\mathcal{H}^{*}} \cdot\|A \otimes y\|_{\mathcal{H}}=\|\phi\|_{\mathcal{H}^{*}} \cdot \sup _{y \in B_{F}}\|A \otimes y\|_{\mathcal{H}} \\
& \leq\|\phi\|_{\mathcal{H}^{*}} \cdot \sup _{y \in B_{F}}\|A\| \cdot\|y\|=\|\phi\|_{\mathcal{H}^{*}} \cdot\|A\|,
\end{aligned}
$$

para todo $A \in \mathcal{L}\left(E_{1}, \ldots, E_{n}\right)$. Isso prova que $\mathcal{B}_{n}(\phi) \in \mathcal{L}\left(\mathcal{L}\left(E_{1}, \ldots, E_{n}\right) ; F^{*}\right)$. Mais uma vez, a linearidade $\mathcal{B}_{n}$ é clara, e de

$$
\begin{aligned}
\left\|\mathcal{B}_{n}(\phi)\right\| & =\sup _{A \in B_{\mathcal{L}\left(E_{1}, \ldots, E_{n}\right)}}\left\|\mathcal{B}_{n}(\phi)(A)\right\| \\
& =\sup _{A \in B_{\mathcal{L}\left(E_{1}, \ldots, E_{n}\right), y \in B_{F}}}\left|\mathcal{B}_{n}(\phi)(A)(y)\right|=\sup _{A, y}|\phi(A \otimes y)| \\
& \leq \sup _{A \in B_{\mathcal{L}\left(E_{1}, \ldots, E_{n}\right), y \in B_{F}}}\|\phi\|_{\mathcal{H}^{*}} \cdot\|A \otimes y\|_{\mathcal{H}} \\
& =\|\phi\|_{\mathcal{H}^{*}} \cdot \sup _{A \in B_{\mathcal{L}\left(E_{1}, \ldots, E_{n}\right)}, y \in B_{F}}\|A \otimes y\|_{\mathcal{H}} \\
& \leq\|\phi\|_{\mathcal{H}^{*}} \cdot \sup _{A \in B_{\mathcal{L}\left(E_{1}, \ldots, E_{n}\right)}, y \in B_{F}}\|A\| \cdot\|y\|=\|\phi\|_{\mathcal{H}^{*}},
\end{aligned}
$$

para todo $\phi \in\left[\mathcal{H}\left(E_{1}, \ldots, E_{n} ; F\right)\right]^{*}$, segue sua continuidade. Mais ainda, a desigualdade $\left\|\mathcal{B}_{n}(\phi)\right\| \leq\|\phi\|_{\mathcal{H}^{*}}$ que acabamos de provar, garante que $\left\|\mathcal{B}_{n}\right\| \leq 1$.

(b) Suponhamos que o subespaço dos operadores de posto finito seja $\|\cdot\|_{\mathcal{H}}$-denso em $\mathcal{H}\left(E_{1}, \ldots, E_{n} ; F\right)$ e seja $\phi \in \operatorname{ker}\left(\mathcal{B}_{n}\right)$. Então, para $B=\sum_{j=1}^{k} A_{j} \otimes y_{j} \in \mathcal{L}_{\mathcal{F}}\left(E_{1}, \ldots, E_{n} ; F\right)$,

$$
\phi(B)=\phi\left(\sum_{j=1}^{k} A_{j} \otimes y_{j}\right)=\sum_{j=1}^{k} \phi\left(A_{j} \otimes y_{j}\right)=\sum_{j=1}^{k} \mathcal{B}_{n}(\phi)\left(A_{j}\right)\left(y_{j}\right)=0 .
$$


Logo, $\phi$ se anula no subespaço denso dos operadores de posto finito. Como $\phi$ é contínuo, segue que $\phi=0, \operatorname{logo} \mathcal{B}_{n}$ é injetora.

Reciprocamente, suponhamos que $\mathcal{B}_{n}$ seja injetora. Tome $\phi \in\left[\mathcal{H}\left(E_{1}, \ldots, E_{n} ; F\right)\right]^{*}$ tal que $\phi(B)=0$ para todo $B \in \mathcal{L}_{\mathcal{F}}\left(E_{1}, \ldots, E_{n} ; F\right)$. Então,

$$
\mathcal{B}_{n}(\phi)(A)(y)=\phi(A \otimes y)=0
$$

para todos $A \in \mathcal{L}\left(E_{1}, \ldots, E_{n}\right)$ e $y \in F$, e portanto $\mathcal{B}_{n}(\phi)=0$. Segue da injetividade de $\mathcal{B}_{n}$ que $\phi=0$. Pela Proposição 1.1.8, obtemos que o subespaço dos operadores de posto finito é $\|\cdot\|_{\mathcal{H}^{-}}$denso em $\mathcal{H}\left(E_{1}, \ldots, E_{n} ; F\right)$.

O operador $\mathcal{B}_{n}$ é chamado de hiper-transformada de Borel. No caso $n=1$, ele coincide com a transformada de Borel linear, isto é, $\mathcal{B}_{1}=\beta$.

Exemplo 3.1.2. Seja $\left(\mathcal{H},\|\cdot\|_{\mathcal{H}}\right)$ um hiper-ideal de Banach de operadores multilineares. Então, $\left(\mathcal{H}\left(E_{1}, \ldots, E_{n} ; F\right),\|\cdot\|_{\mathcal{H}}\right)$ satisfaz as condições da proposição anterior para todo $n$ e quaisquer espaços de Banach $E_{1}, \ldots, E_{n}$ e $F \operatorname{com}\|A \otimes y\|_{\mathcal{H}}=\|A\| \cdot\|y\|$ para todos $A \in \mathcal{L}\left(E_{1}, \ldots, E_{n}\right)$ e $y \in F($ veja $[72])$.

Uma vez que nosso objetivo é representar funcionais lineares em espaços de operadores multilineares, devemos primeiro considerar quando $\mathcal{B}_{n}$ é um isomorfismo sobre sua imagem com respeito à norma usual de operadores. No próximo teorema provamos que a única classe de operadores multilineares para a qual a hiper-transformada de Borel pode ser um isomorfismo sobre sua imagem com respeito à norma usual é a classe dos operadores hiper-nucleares. E, mais ainda, nesse caso ela é sobrejetora.

Teorema 3.1.3. Seja $\mathcal{H}\left(E_{1}, \ldots, E_{n} ; F\right)$ um subespaço de $\mathcal{L}\left(E_{1}, \ldots, E_{n} ; F\right)$ munido de uma norma completa $\|\cdot\|_{\mathcal{H}}$, contendo os operadores multilineares de posto finito e tal que $\|\cdot\| \leq\|\cdot\|_{\mathcal{H}} e\|B \otimes y\|_{\mathcal{H}} \leq\|B\| \cdot\|y\|$ para todos $B \in \mathcal{L}\left(E_{1}, \ldots, E_{n}\right)$ e $y \in F$. Suponha que a hiper-transformada de Borel

$$
\begin{gathered}
\mathcal{B}_{n}:\left(\mathcal{H}\left(E_{1}, \ldots, E_{n} ; F\right),\|\cdot\|_{\mathcal{H}}\right)^{*} \longrightarrow\left(\mathcal{L}\left(\mathcal{L}\left(E_{1}, \ldots, E_{n}\right) ; F^{*}\right),\|\cdot\|\right), \\
\mathcal{B}_{n}(\phi)(B)(y)=\phi(B \otimes y),
\end{gathered}
$$

seja um isomorfismo sobre sua imagem. Então, $\mathcal{H}\left(E_{1}, \ldots, E_{n} ; F\right)=\mathcal{L}_{\mathcal{H N}}\left(E_{1}, \ldots E_{n} ; F\right)$, $\mathcal{B}_{n}$ é sobrejetora e as normas $\|\cdot\|_{\mathcal{H}} e\|\cdot\|_{\mathcal{H N}}$ são equivalentes. 
Demonstração. Considere $A \in \mathcal{L}_{\mathcal{H N}}\left(E_{1}, \ldots, E_{n} ; F\right)$ e $\varepsilon>0$. Pelo Lema 2.1.7, podemos tomar uma representação hiper-nuclear

$$
A=\sum_{j=1}^{\infty} \lambda_{j} B_{j} \otimes y_{j}
$$

tal que

$$
\sum_{j=1}^{\infty}\left|\lambda_{j}\right| \cdot\left\|B_{j}\right\| \cdot\left\|y_{j}\right\| \leq(1+\varepsilon)\|A\|_{\mathcal{H N}}
$$

Para $m \in \mathbb{N}$, consideremos

$$
A_{m}:=\sum_{j=1}^{m} \lambda_{j} B_{j} \otimes y_{j} \in \mathcal{L}_{\mathcal{F}}\left(E_{1}, \ldots, E_{n} ; F\right) \subseteq \mathcal{H}\left(E_{1}, \ldots, E_{n} ; F\right) .
$$

Como $\sum_{j=1}^{\infty}\left|\lambda_{j}\right| \cdot\left\|B_{j}\right\| \cdot\left\|y_{j}\right\|<\infty$, existe $m_{0} \in \mathbb{N}$ tal que $\sum_{j=m+1}^{\infty}\left|\lambda_{j}\right| \cdot\left\|B_{j}\right\| \cdot\left\|y_{j}\right\|<\varepsilon$ para todo $m \geq m_{0}$. Então, para $m>k \geq m_{0}$,

$$
\begin{aligned}
\left\|A_{m}-A_{k}\right\|_{\mathcal{H}} & =\left\|\sum_{j=1}^{m} \lambda_{j} B_{j} \otimes y_{j}-\sum_{j=1}^{k} \lambda_{j} B_{j} \otimes y_{j}\right\|_{\mathcal{H}} \\
& =\left\|\sum_{j=k+1}^{m} \lambda_{j} B_{j} \otimes y_{j}\right\|_{\mathcal{H}} \\
& \leq \sum_{j=k+1}^{m}\left\|\lambda_{j} B_{j} \otimes y_{j}\right\|_{\mathcal{H}} \\
& \leq \sum_{j=k+1}^{m}\left|\lambda_{j}\right| \cdot\left\|B_{j}\right\| \cdot\left\|y_{j}\right\|<\varepsilon,
\end{aligned}
$$

o que mostra que $\left(A_{m}\right)_{m=1}^{\infty}$ é uma sequência de Cauchy em $\mathcal{H}\left(E_{1}, \ldots, E_{n} ; F\right)$. Logo, existe $C \in \mathcal{H}\left(E_{1}, \ldots, E_{n} ; F\right)$ tal que $A_{m} \longrightarrow C$ em $\mathcal{H}\left(E_{1}, \ldots, E_{n} ; F\right)$. Como $\|\cdot\| \leq\|\cdot\|_{\mathcal{H}}$, concluímos que $A_{m} \longrightarrow C$ em $\mathcal{L}\left(E_{1}, \ldots, E_{n} ; F\right)$.

Agora notemos que, para todo $m$,

$$
\begin{aligned}
\left\|A_{m}-A\right\| & =\left\|\sum_{j=m+1}^{\infty} \lambda_{j} B_{j} \otimes y_{j}\right\| \\
& =\sup _{\left\|x_{j}\right\| \leq 1}\left\|\sum_{j=m+1}^{\infty} \lambda_{j} B_{j} \otimes y_{j}\left(x_{1}, \ldots, x_{n}\right)\right\|
\end{aligned}
$$




$$
\begin{aligned}
& \leq \sup _{\left\|x_{j}\right\| \leq 1} \sum_{j=m+1}^{\infty}\left|\lambda_{j}\right| \cdot\left\|B_{j}\right\| \cdot\left\|y_{j}\right\| \cdot\left\|x_{1}\right\| \cdots\left\|x_{n}\right\| \\
& =\sum_{j=m+1}^{\infty}\left|\lambda_{j}\right| \cdot\left\|B_{j}\right\| \cdot\left\|y_{j}\right\| \stackrel{m \rightarrow \infty}{\longrightarrow} 0,
\end{aligned}
$$

pois a série $\sum_{j=1}^{\infty}\left|\lambda_{j}\right| \cdot\left\|B_{j}\right\| \cdot\left\|y_{j}\right\|$ é convergente. Isso mostra que $A_{m} \longrightarrow A \operatorname{em~} \mathcal{L}\left(E_{1}, \ldots, E_{n} ; F\right)$. Portanto, $A=C \in \mathcal{H}\left(E_{1}, \ldots, E_{n} ; F\right)$. Além disso, usando novamente que $A_{m} \longrightarrow C$ em $\mathcal{H}\left(E_{1}, \ldots, E_{n} ; F\right)$,

$$
\begin{aligned}
\|A\|_{\mathcal{H}} & =\|C\|_{\mathcal{H}}=\lim _{m}\left\|A_{m}\right\|_{\mathcal{H}}=\lim _{m}\left\|\sum_{j=1}^{m} \lambda_{j} B_{j} \otimes y_{j}\right\|_{\mathcal{H}} \\
& \leq \lim _{m} \sum_{j=1}^{m}\left|\lambda_{j}\right| \cdot\left\|B_{j}\right\| \cdot\left\|y_{j}\right\|=\sum_{j=1}^{\infty}\left|\lambda_{j}\right| \cdot\left\|B_{j}\right\| \cdot\left\|y_{j}\right\| \leq(1+\varepsilon)\|A\|_{\mathcal{H} \mathcal{N}} .
\end{aligned}
$$

Fazendo $\varepsilon \longrightarrow 0^{+}$, obtemos $\|A\|_{\mathcal{H}} \leq\|A\|_{\mathcal{H N}}$. Em particular, a inclusão

$$
i: \mathcal{L}_{\mathcal{H N}}\left(E_{1}, \ldots, E_{n} ; F\right) \hookrightarrow \mathcal{H}\left(E_{1}, \ldots, E_{n} ; F\right)
$$

é um operador linear contínuo.

Provemos agora a inclusão oposta. Segue das hipóteses e do Lema 2.1.7 que

$$
T: \mathcal{L}\left(E_{1}, \ldots, E_{n}\right) \times F \longrightarrow \mathcal{L}_{\mathcal{H N}}\left(E_{1}, \ldots, E_{n} ; F\right), T(B, y)=B \otimes y
$$

é um operador bilinear e contínuo. Pelo Teorema 1.2.13, existe um único operador linear contínuo

$$
T_{L}: \mathcal{L}\left(E_{1}, \ldots, E_{n}\right) \widehat{\otimes}_{\pi} F \longrightarrow \mathcal{L}_{\mathcal{H N}}\left(E_{1}, \ldots, E_{n} ; F\right)
$$

tal que

$$
T_{L}(B \otimes y)=T(B, y)=B \otimes y,
$$

para todos $B \in \mathcal{L}\left(E_{1}, \ldots, E_{n}\right)$ e $y \in F$.

Considere os operadores

$$
\begin{gathered}
V_{1}:\left(\mathcal{L}\left(E_{1}, \ldots, E_{n}\right) \widehat{\otimes}_{\pi} F\right)^{*} \longrightarrow \mathcal{L}\left(\mathcal{L}\left(E_{1}, \ldots, E_{n}\right), F ; \mathbb{K}\right), V_{1}(\varphi)(B, y)=\varphi(B \otimes y) e \\
V_{2}: \mathcal{L}\left(\mathcal{L}\left(E_{1}, \ldots, E_{n}\right), F ; \mathbb{K}\right) \longrightarrow \mathcal{L}\left(\mathcal{L}\left(E_{1}, \ldots, E_{n}\right) ; F^{*}\right), V_{2}(\xi)(B)(y)=\xi(B, y) .
\end{gathered}
$$

Pelo Teorema 1.2.13, $V_{1}$ é um isomorfismo isométrico e, pela Proposição 1.2.6(b), $V_{2}$ também é um isomorfismo isométrico. Para todos $B \in \mathcal{L}\left(E_{1}, \ldots, E_{n}\right)$ e $y \in F$,

$$
\left(V_{2} \circ V_{1}\right)(\varphi)(B)(y)=V_{2}\left(V_{1}(\varphi)\right)(B)(y)=V_{1}(\varphi)(B, y)=\varphi(B \otimes y) .
$$


Assim,

$$
V_{2} \circ V_{1} \circ T_{L}^{*} \circ i^{*}:\left[\mathcal{H}\left(E_{1}, \ldots, E_{n} ; F\right)\right]^{*} \longrightarrow \mathcal{L}\left(\mathcal{L}\left(E_{1}, \ldots, E_{n}\right) ; F^{*}\right)
$$

é um operador linear contínuo e, para todos $\phi \in\left[\mathcal{H}\left(E_{1}, \ldots, E_{n} ; F\right)\right]^{*}, B \in \mathcal{L}\left(E_{1}, \ldots, E_{n}\right)$ e $y \in F$,

$$
\begin{aligned}
\left(V_{2} \circ V_{1} \circ T_{L}^{*} \circ i^{*}\right)(\phi)(B)(y) & =\left(V_{2} \circ V_{1}\right)\left(\left(i \circ T_{L}\right)^{*}(\phi)\right)(B)(y) \\
& =\left(i \circ T_{L}\right)^{*}(\phi)(B \otimes y) \\
& =\phi\left(i \circ T_{L}\right)(B \otimes y) \\
& =\phi(B \otimes y)=\mathcal{B}_{n}(\phi)(B)(y) .
\end{aligned}
$$

Isso mostra que $V_{2} \circ V_{1} \circ T_{L}^{*} \circ i^{*}=\mathcal{B}_{n}$ é um isomorfismo sobre sua imagem, por hipótese. Em particular, $V_{2} \circ V_{1} \circ T_{L}^{*} \circ i^{*}$ é injetivo. Como $V_{2} \circ V_{1}$ é um isomorfismo, concluímos que $T_{L}^{*} \circ i^{*}$ é injetivo. Consideremos agora o isomorfismo $\left(V_{2} \circ V_{1}\right)^{-1}$ :

$$
\left(V_{2} \circ V_{1}\right)^{-1}: \mathcal{L}\left(\mathcal{L}\left(E_{1}, \ldots, E_{n}\right) ; F^{*}\right) \longrightarrow\left(\mathcal{L}\left(E_{1}, \ldots, E_{n}\right) \widehat{\otimes}_{\pi} F\right)^{*}
$$

Chamando de $\psi$ a restrição de $\left(V_{2} \circ V_{1}\right)^{-1}$ à imagem de $\mathcal{B}_{n}$, segue que

$$
\psi: \mathcal{B}_{n}\left(\mathcal{H}\left(E_{1}, \ldots, E_{n} ; F\right)^{*}\right) \longrightarrow\left(V_{2} \circ V_{1}\right)^{-1}\left(\mathcal{B}_{n}\left(\mathcal{H}\left(E_{1}, \ldots, E_{n} ; F\right)^{*}\right)\right)
$$

é um isomorfismo. Como $\mathcal{B}_{n}$ é isomorfismo sobre sua imagem, $\mathcal{B}_{n}\left(\mathcal{H}\left(E_{1}, \ldots, E_{n} ; F\right)^{*}\right)$ é um espaço de Banach. Assim, $\psi\left(\mathcal{B}_{n}\left(\mathcal{H}\left(E_{1}, \ldots, E_{n} ; F\right)^{*}\right)\right)$ também é um espaço de Banach e, portanto, um subespaço fechado de $\left(\mathcal{L}\left(E_{1}, \ldots, E_{n}\right) \widehat{\otimes}_{\pi} F\right)^{*}$.

Para todo $\phi \in\left[\mathcal{H}\left(E_{1}, \ldots, E_{n} ; F\right)\right]^{*}$,

$$
\left(T_{L}^{*} \circ i^{*}\right)(\phi)=\left(V_{2} \circ V_{1}\right)^{-1} \circ \mathcal{B}_{n}(\phi)=\psi\left(\mathcal{B}_{n}(\phi)\right)
$$

e portanto

$$
\left(i \circ T_{L}\right)^{*}\left(\mathcal{H}\left(E_{1}, \ldots, E_{n} ; F\right)^{*}\right)=\psi\left(\mathcal{B}_{n}\left(\mathcal{H}\left(E_{1}, \ldots, E_{n} ; F\right)^{*}\right)\right)
$$

é um subespaço fechado $\left(\mathcal{L}\left(E_{1}, \ldots, E_{n}\right) \widehat{\otimes}_{\pi} F\right)^{*}$. Como $\left(i \circ T_{L}\right)^{*}$ é injetor e tem imagem fechada, pelo Teorema da Aplicação Aberta concluímos que $\left(i \circ T_{L}\right)^{*}$ é um isomorfismo sobre sua imagem. Do Teorema 1.1.7 segue que $i \circ T_{L}$ é sobrejetor, donde segue claramente que $i$ é sobrejetora. Isso prova que $\mathcal{L}_{\mathcal{H N}}\left(E_{1}, \ldots, E_{n} ; F\right)=\mathcal{H}\left(E_{1}, \ldots, E_{n} ; F\right)$.

Já sabíamos que $i: \mathcal{L}_{\mathcal{H N}} \hookrightarrow \mathcal{H}$ é linear, contínuo e injetor; e agora sabemos que é bijetor. Pelo Teorema da Aplicação Aberta concluímos que $i$ é um isomorfismo, e assim as normas $\|\cdot\|_{\mathcal{H N}}$ e $\|\cdot\|_{\mathcal{H}}$ são equivalentes. 
Finalmente, vejamos que $\mathcal{B}_{n}$ é sobrejetora. Dado $v \in \mathcal{L}\left(\mathcal{L}\left(E_{1}, \ldots, E_{n}\right) ; F^{*}\right)$, consideremos $A_{L}: \mathcal{L}\left(E_{1}, \ldots, E_{n}\right) \widehat{\otimes}_{\pi} F \longrightarrow \mathbb{K}$ a linearização do operador bilinear contínuo

$$
A: \mathcal{L}\left(E_{1}, \ldots, E_{n}\right) \times F \longrightarrow \mathbb{K}, A(B, y)=v(B)(y)
$$

Usando o fato de que a correspondência

$$
\mathcal{L}_{\mathcal{F}}\left(E_{1}, \ldots, E_{n} ; F\right) \longleftrightarrow \mathcal{L}\left(E_{1}, \ldots, E_{n}\right) \otimes F
$$

é um isomorfismo entre espaços vetoriais, podemos considerar a restrição $\varphi$ de $A_{L}$ a

$$
\mathcal{L}\left(E_{1}, \ldots, E_{n}\right) \otimes F \subseteq \mathcal{L}_{\mathcal{H N}}\left(E_{1}, \ldots, E_{n} ; F\right)
$$

isto é,

$$
\varphi:\left(\mathcal{L}\left(E_{1}, \ldots, E_{n}\right) \otimes F,\|\cdot\|_{\mathcal{H N}}\right) \longrightarrow \mathbb{K}, \varphi(B)=A_{L}(B) .
$$

É claro que $\varphi$ é linear. Provemos que é contínuo. Seja $B \in \mathcal{L}\left(E_{1}, \ldots, E_{n}\right) \otimes F$. Dado $\varepsilon>0$, pelo Lema 2.1.7 podemos tomar $B=\sum_{j=1}^{\infty} \lambda_{j} B_{j} \otimes y_{j}$ uma representação hiper-nuclear de $B$ tal que $\sum_{j=1}^{\infty}\left|\lambda_{j}\right| \cdot\left\|B_{j}\right\| \cdot\left\|y_{j}\right\| \leq(1+\varepsilon)\|B\|_{\mathcal{H N}}$. Como

$$
\sum_{j=1}^{\infty} \pi\left(\lambda_{j} B_{j} \otimes y_{j}\right)=\sum_{j=1}^{\infty}\left|\lambda_{j}\right| \cdot\left\|B_{j} \otimes y_{j}\right\|=\sum_{j=1}^{\infty}\left|\lambda_{j}\right| \cdot\left\|B_{j}\right\| \cdot\left\|y_{j}\right\|<+\infty
$$

a série $\sum_{j=1}^{\infty} \lambda_{j} B_{j} \otimes y_{j}$ é absolutamente convergente em $\mathcal{L}\left(E_{1}, \ldots, E_{n}\right) \widehat{\otimes}_{\pi} F$, e portanto convergente. Assim, a convergência $B=\sum_{j=1}^{\infty} \lambda_{j} B_{j} \otimes y_{j}$ se dá na norma $\pi$ de $\mathcal{L}\left(E_{1}, \ldots, E_{n}\right) \widehat{\otimes}_{\pi} F$. Conforme mostrado no início desta demonstração, essa convergência também se dá na norma $\|\cdot\|_{\mathcal{H N}}$. Da continuidade de $A_{L}$ temos

$$
\begin{aligned}
|\varphi(B)|=\left|A_{L}(B)\right| & =\left|A_{L}\left(\sum_{j=1}^{\infty} \lambda_{j} B_{j} \otimes y_{j}\right)\right| \\
& \leq\left\|A_{L}\right\| \cdot \pi\left(\sum_{j=1}^{\infty} \lambda_{j} B_{j} \otimes y_{j}\right) \\
& \leq\left\|A_{L}\right\| \cdot \sum_{j=1}^{\infty} \pi\left(\lambda_{j} B_{j} \otimes y_{j}\right) \\
& =\left\|A_{L}\right\| \cdot \sum_{j=1}^{\infty}\left|\lambda_{j}\right| \cdot\left\|B_{j}\right\| \cdot\left\|y_{j}\right\|
\end{aligned}
$$




$$
\leq(1+\varepsilon)\left\|A_{L}\right\| \cdot\|B\|_{\mathcal{H N}}
$$

Isso mostra que o funcional $\varphi$ é contínuo com respeito à norma $\|\cdot\|_{\mathcal{H N}}$, isto é, $\varphi \in\left(\mathcal{L}\left(E_{1}, \ldots, E_{n}\right) \otimes F,\|\cdot\|_{\mathcal{H N}}\right)^{*}$. Usando mais uma vez o que mostramos no início da demonstração, que somas parciais de representações hiper-nucleares convergem na norma $\|\cdot\|_{\mathcal{H N}}$ para o operador hiper-nuclear, segue que $\mathcal{L}\left(E_{1}, \ldots, E_{n}\right) \otimes F$ é denso em $\mathcal{L}_{\mathcal{H N}}\left(E_{1}, \ldots, E_{n} ; F\right)$. Seja então $\tilde{\varphi}$ a (única) extensão linear e contínua de $\varphi$ a $\mathcal{L}_{\mathcal{H N}}\left(E_{1}, \ldots, E_{n} ; F\right)$. Para todos $B \in \mathcal{L}\left(E_{1}, \ldots, E_{n}\right)$ e $y \in F$,

$$
\mathcal{B}_{n}(\tilde{\varphi})(B)(y)=\tilde{\varphi}(B \otimes y)=\varphi(B \otimes y)=A_{L}(B \otimes y)=A(B, y)=v(B)(y)
$$

isto é, $\mathcal{B}_{n}(\tilde{\varphi})=v$, o que prova a sobrejetividade de $\mathcal{B}_{n}$ e completa a demonstração.

Veremos na próxima seção que, sob condições envolvendo a propriedade da aproximação, de fato a hiper-transformada de Borel (3.1) é um isomorfismo isométrico.

O teorema acima diz que, para representar funcionais lineares em $\mathcal{H}\left(E_{1}, \ldots, E_{n} ; F\right)$, onde a classe $\mathcal{H}$ é distinta da classe dos operadores multilineares hiper-nucleares, é necessário substituir a norma usual em $\mathcal{B}_{n}\left(\mathcal{H}\left(E_{1}, \ldots, E_{n} ; F\right)^{*}\right) \subseteq \mathcal{L}\left(\mathcal{L}\left(E_{1}, \ldots, E_{n}\right) ; F^{*}\right)$ por alguma norma conveniente. O caminho mais óbvio é pensar em normas de ideais de Banach de operadores lineares, mas a noção mais geral que introduzimos a seguir se mostrará posteriormente mais adequada.

Definição 3.1.4. Um semi-ideal de operadores à esquerda é uma correspondência $\alpha$ que, a cada par de espaços de Banach $(E, F)$, associa um subespaço vetorial $\mathcal{L}_{\alpha}\left(E ; F^{*}\right)$ de $\mathcal{L}\left(E ; F^{*}\right)$ munido com uma norma completa $\|\cdot\|_{\alpha}$ satisfazendo a propriedade de ideal à esquerda: se $G$ é espaço de Banach, $v \in \mathcal{L}(E ; F)$ e $T \in \mathcal{L}_{\alpha}\left(F ; G^{*}\right)$, então $T \circ v \in \mathcal{L}_{\alpha}\left(E ; G^{*}\right)$ $\mathrm{e}$

$$
\|T \circ v\|_{\alpha} \leq\|T\|_{\alpha} \cdot\|v\| \text {. }
$$

Denotaremos por $\mathcal{L}_{\alpha}\left(E ; F^{*}\right)$ o espaço de Banach $\left(\mathcal{L}_{\alpha}\left(E ; F^{*}\right),\|\cdot\|_{\alpha}\right)$.

Observe que essa noção é mais geral que ideais de operadores em dois sentidos: (i) não é preciso definir $\mathcal{L}(E ; F)$ para todos $E$ e $F$, basta fazê-lo no caso em que $F$ é um espaço dual; (ii) a propriedade de ideal à direita não é necessária. Esses dois graus de generalidade serão muito importantes mais adiante.

De toda forma, é óbvio que:

Exemplo 3.1.5. Todo ideal de Banach de operadores é um semi-ideal à esquerda. 
Exemplo 3.1.6. Vejamos que, para todo $p \geq 1$, a classe dos operadores lineares quase$\tau(p)$-somantes (Definição 3.0.3) é um semi-ideal à esquerda. De fato, $\left(\mathcal{L}_{q \tau(p)}\left(E ; F^{*}\right), \|\right.$. $\left.\|_{q \tau(p)}\right)$ é espaço de Banach por definição e, dados $v \in \mathcal{L}(E ; F)$ e $T \in \mathcal{L}_{q \tau(p)}\left(F ; G^{*}\right)$ existe $C \geq 0$ tal que

$$
\left(\sum_{j=1}^{m}\left|T\left(z_{j}\right)\left(w_{j}\right)\right|^{p}\right)^{\frac{1}{p}} \leq C \cdot \sup _{z^{*} \in B_{F^{*}}, w^{*} \in B_{G^{*}}}\left(\sum_{j=1}^{m}\left|z^{*}\left(z_{j}\right) w^{*}\left(w_{j}\right)\right|^{p}\right)^{\frac{1}{p}}
$$

para todos $m \in \mathbb{N}, z_{1}, \ldots, z_{m} \in F$ e $w_{1}, \ldots, w_{m} \in G$. Segue que

$$
\begin{aligned}
& \left(\sum_{j=1}^{m}\left|(T \circ v)\left(x_{j}\right)\left(w_{j}\right)\right|^{p}\right)^{\frac{1}{p}}=\left(\sum_{j=1}^{m}\left|\left(T\left(v\left(x_{j}\right)\right)\right)\left(w_{j}\right)\right|^{p}\right)^{\frac{1}{p}} \\
& \leq C \cdot \sup _{z^{*} \in B_{F^{*}}, w^{*} \in B_{G^{*}}}\left(\sum_{j=1}^{m}\left|z^{*}\left(v\left(x_{j}\right)\right) w^{*}\left(w_{j}\right)\right|^{p}\right)^{\frac{1}{p}} \\
& =C \cdot \sup _{z^{*} \in B_{F^{*}}, w^{*} \in B_{G^{*}}}\left(\sum_{j=1}^{m}\left|\left(z^{*} \circ v\right)\left(x_{j}\right) w^{*}\left(w_{j}\right)\right|^{p}\right)^{\frac{1}{p}} \\
& (v \neq 0)=C \cdot\|v\| \cdot \sup _{z^{*} \in B_{F^{*}}, w^{*} \in B_{G^{*}}}\left(\sum_{j=1}^{m}\left|\left(z^{*} \circ \frac{v}{\|v\|}\right)\left(x_{j}\right) w^{*}\left(w_{j}\right)\right|^{p}\right)^{\frac{1}{p}} \\
& \leq C \cdot\|v\| \cdot \sup _{x^{*} \in B_{E^{*}, w^{*} \in B_{G^{*}}}}\left(\sum_{j=1}^{m}\left|x^{*}\left(x_{j}\right) w^{*}\left(w_{j}\right)\right|^{p}\right)^{\frac{1}{p}}
\end{aligned}
$$

para todos $m \in \mathbb{N}, x_{1}, \ldots, x_{m} \in E$ e $w_{1}, \ldots, w_{m} \in G$. Isso prova que $(T \circ v) \in$ $\mathcal{L}_{q \tau(p)}\left(E ; G^{*}\right)$. Tomando o ínfimo sobre as constantes $C$ obtemos a desigualdade $\| T \circ$ $v\left\|_{q \tau(p)} \leq\right\| T\left\|_{q \tau(p)} \cdot\right\| v \|$.

Vejamos uma classe que satisfaz a propriedade de ideal à esquerda mas não à direita.

Exemplo 3.1.7. Um operador linear $u: E \longrightarrow F^{*}$ é $w^{*}$-sequencialmente compacto se, para toda sequência limitada $\left(x_{n}\right)_{n=1}^{\infty}$ em $E,\left(u\left(x_{n}\right)\right)_{n=1}^{\infty}$ admite uma subsequência $w^{*}$ convergente em $F^{*}$.

Como operadores contínuos transformam sequências limitadas em sequências limitadas, a classe dos operadores lineares $w^{*}$-sequencialmente compactos satisfaz a propriedade de ideal à esquerda. E como nem sempre operadores lineares contínuos transformam sequências $w^{*}$-convergentes em sequências $w^{*}$-convergentes, é de se esperar que essa classe não satisfaça a propriedade de ideal à direita. De fato, isso está provado em [16, Proposition 3.3.2]. 
Lema 3.1.8. Seja a um semi-ideal de operadores à esquerda. Se v: E $\longrightarrow F$ é um isomorfismo (isométrico), então, para todo espaço de Banach $G$, o operador de composição

$$
J_{v}: \mathcal{L}_{\alpha}\left(F ; G^{*}\right) \longrightarrow \mathcal{L}_{\alpha}\left(E ; G^{*}\right), J_{v}(T)=T \circ v
$$

é também um isomorfismo (isométrico).

Demonstração. Como $\alpha$ é um semi-ideal à esquerda, temos $J_{v}(T)=T \circ v \in \mathcal{L}_{\alpha}\left(E ; G^{*}\right)$ para todo $T \in \mathcal{L}_{\alpha}\left(F ; G^{*}\right)$, o que mostra que $J_{v}$ está bem definido. É fácil ver que $J_{v}$ é linear.

Façamos o caso em que $v$ é um isomorfismo isométrico. Dado $u \in \mathcal{L}_{\alpha}\left(E ; G^{*}\right)$, considere o operador

$$
T_{u}:=u \circ v^{-1}: F \longrightarrow G^{*} .
$$

Como $v$ é um isomorfismo, $T_{u}$ está bem definido, e como $\alpha$ é um semi-ideal à esquerda, $T_{u} \in \mathcal{L}_{\alpha}\left(F ; G^{*}\right)$. De

$$
J_{v}\left(T_{u}\right)=T_{u} \circ v=u \circ v^{-1} \circ v=u,
$$

concluímos que $J_{v}$ é sobrejetora. E de

$$
\begin{aligned}
\left\|J_{v}(T)\right\|_{\alpha} & =\|T \circ v\|_{\alpha} \leq\|T\|_{\alpha} \cdot\|v\|=\|T\|_{\alpha}=\left\|T \circ v \circ v^{-1}\right\|_{\alpha} \\
& \leq\|(T \circ v)\|_{\alpha} \cdot\left\|v^{-1}\right\|=\|T \circ v\|_{\alpha}=\left\|J_{v}(T)\right\|_{\alpha},
\end{aligned}
$$

segue que $\left\|J_{v}(T)\right\|_{\alpha}=\|T\|_{\alpha}$, provando que $J_{u}$ é uma imersão isométrica, portanto um isomorfismo isométrico.

\subsection{Multi-ideais de composição}

Nesta seção mostraremos como representações lineares implicam em representações multilineares em ideais de composição.

Sejam $\mathcal{I}$ um ideal de Banach de operadores e $\alpha$ um semi-ideal à esquerda. Suponha que a transformada de Borel linear,

$$
\mathcal{B}:=\beta:\left(\mathcal{I}(E ; F),\|\cdot\|_{\mathcal{I}}\right)^{*} \longrightarrow \mathcal{L}_{\alpha}\left(E^{*} ; F^{*}\right), \mathcal{B}(\phi)\left(x^{*}\right)(y)=\phi\left(x^{*} \otimes y\right),
$$

seja um isomorfismo isométrico, isto é, representa os funcionais em $[\mathcal{I}(E, F)]^{*}$ como operadores de $\mathcal{L}_{\alpha}\left(E^{*} ; F^{*}\right)$. O próximo teorema diz que, neste caso, a hiper-transformada de Borel representa funcionais em $\left[\mathcal{I} \circ \mathcal{L}\left(E_{1}, \ldots, E_{n} ; F\right)\right]^{*}$, onde $\mathcal{I} \circ \mathcal{L}$ é o multi-ideal de 
composição. Como $\mathcal{I} \circ \mathcal{L}$ é um hiper-ideal (Teorema 1.5.2 ou [20]), isso está de acordo com os teoremas que já provamos sobre a hiper-transformada de Borel.

Por uma propriedade geométrica de espaços de Banach entendemos uma propriedade $P$ na classe de todos os espaços de Banach sobre $\mathbb{K}$ que é invariante por isomorfismos isométricos, isto é: se $E$ tem $P$ e $F$ é isomorfo isometricamente a $E$, então $F$ tem $P$. Em particular, toda propriedade topológica, isto é, uma propriedade que é invariante por isomorfismos topológicos, é uma propriedade geométrica.

Teorema 3.2.1. Sejam $\left(\mathcal{I},\|\cdot\|_{\mathcal{I}}\right)$ um ideal de Banach de operadores, $\alpha$ um semi-ideal à esquerda e $P_{1}, P_{2}$ propriedades geométricas de espaços de Banach. As seguintes afirmações são equivalentes:

(a) Para todos espaços de Banach $E$ e $F$ tais que $E^{*}$ tem $P_{1}$ e F tem $P_{2}$, a transformada de Borel linear

$$
\mathcal{B}:\left(\mathcal{I}(E ; F),\|\cdot\|_{\mathcal{I}}\right)^{*} \longrightarrow \mathcal{L}_{\alpha}\left(E^{*} ; F^{*}\right), \mathcal{B}(\phi)\left(x^{*}\right)(y)=\phi\left(x^{*} \otimes y\right),
$$

é um isomorfismo isométrico.

(b) Para todo $n \in \mathbb{N}$ e todos espaços de Banach $E_{1}, \ldots, E_{n}$ e $F$ tais que $\mathcal{L}\left(E_{1}, \ldots, E_{n}\right)$ tem $P_{1}$ e F tem $P_{2}$, a hiper-transformada de Borel

$\mathcal{B}_{n}:\left(\mathcal{I} \circ \mathcal{L}\left(E_{1}, \ldots, E_{n} ; F\right),\|\cdot\|_{\mathcal{I} \circ \mathcal{L}}\right)^{*} \longrightarrow \mathcal{L}_{\alpha}\left(\mathcal{L}\left(E_{1}, \ldots, E_{n}\right) ; F^{*}\right), \mathcal{B}_{n}(\phi)(A)(y)=\phi(A \otimes y)$,

é um isomorfismo isométrico.

(c) Para algum $n \geq 2$ e todos espaços de Banach $E_{1}, \ldots, E_{n}$ e $F$ tais que $\mathcal{L}\left(E_{1}, \ldots, E_{n}\right)$ tem $P_{1}$ e F tem $P_{2}$, a hiper-transformada de Borel (3.2) é um isomorfismo isométrico.

Demonstração. (a) $\Longrightarrow$ (b) Seja $n \in \mathbb{N}$ e suponha que $\mathcal{L}\left(E_{1}, \ldots, E_{n}\right)$ tenha $P_{1}$ e que $F$ tenha $P_{2}$. Pelas Proposições 1.4 .8 e 1.4 .9 sabemos que o operador linearização

$$
L: \mathcal{I} \circ \mathcal{L}\left(E_{1}, \ldots, E_{n} ; F\right) \longrightarrow \mathcal{I}\left(E_{1} \hat{\otimes}_{\pi} \cdots \hat{\otimes}_{\pi} E_{n} ; F\right), L(A)=A_{L},
$$

é um isomorfismo isométrico. Segue que o adjunto do isomorfismo inverso,

$$
\left(L^{-1}\right)^{*}:\left[\mathcal{I} \circ \mathcal{L}\left(E_{1}, \ldots, E_{n} ; F\right)\right]^{*} \longrightarrow\left[\mathcal{I}\left(E_{1} \hat{\otimes}_{\pi} \cdots \hat{\otimes}_{\pi} E_{n} ; F\right)\right]^{*}
$$

também é um isomorfismo isométrico. Como o operador linearização $\ell: \mathcal{L}\left(E_{1}, \ldots, E_{n}\right) \longrightarrow$ $\left(E_{1} \hat{\otimes}_{\pi} \cdots \hat{\otimes}_{\pi} E_{n}\right)^{*}$ é um isomorfismo isométrico (Teorema 1.2.13), temos: 
(i) $\left(E_{1} \hat{\otimes}_{\pi} \cdots \hat{\otimes}_{\pi} E_{n}\right)^{*}$ tem $P_{1}$, e portanto a transformada de Borel linear $\mathcal{B}$ é, por hipótese, um isomorfismo isométrico de $\left[\mathcal{I}\left(E_{1} \hat{\otimes}_{\pi} \cdots \hat{\otimes}_{\pi} E_{n} ; F\right)\right]^{*}$ em $\mathcal{L}_{\alpha}\left(\left(E_{1} \hat{\otimes}_{\pi} \cdots \hat{\otimes}_{\pi} E_{n}\right)^{*} ; F^{*}\right)$.

(ii) O operador de composição

$$
\left.J_{\ell}: \mathcal{L}_{\alpha}\left(\left(E_{1} \hat{\otimes}_{\pi} \cdots \hat{\otimes}_{\pi} E_{n}\right)^{*} ; F^{*}\right) \longrightarrow \mathcal{L}_{\alpha}\left(\mathcal{L}\left(E_{1}, \ldots, E_{n}\right) ; F^{*}\right)\right), J_{\ell}(u)=u \circ \ell,
$$

é um isomorfismo isométrico pelo Lema 3.1.8.

É suficiente provar que o diagrama

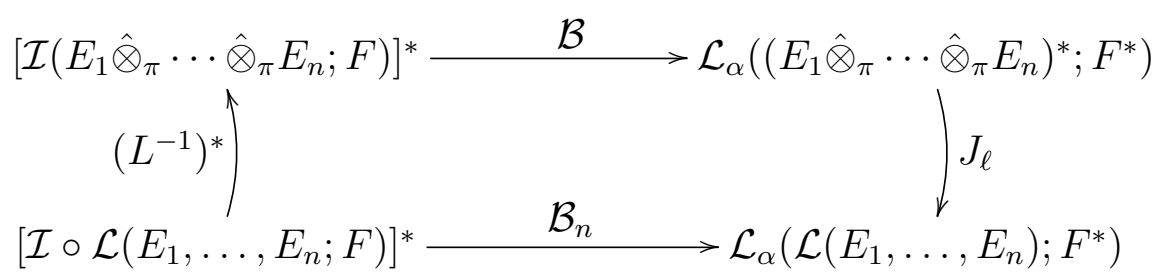

é comutativo: para $\phi \in\left[(\mathcal{I} \circ \mathcal{L})\left(E_{1}, \ldots, E_{n} ; F\right)\right]^{*}, A \in \mathcal{L}\left(E_{1}, \ldots, E_{n}\right)$ e $y \in F$,

$$
\begin{aligned}
\left(J_{\ell} \circ \mathcal{B} \circ\left(L^{-1}\right)^{*}\right)(\phi)(A)(y) & =\left(\left(J_{\ell}\left(\mathcal{B}\left(\left(L^{-1}\right)^{*}(\phi)\right)\right)\right)(A)\right)(y) \\
& =\left(\left(\mathcal{B}\left(\left(L^{-1}\right)^{*}(\phi)\right)\right)\left(A_{L}\right)\right)(y) \\
& =\left(L^{-1}\right)^{*}(\phi)\left(A_{L} \otimes y\right) \\
& =\phi\left(\left(L^{-1}\right)\left(A_{L} \otimes y\right)\right) \\
& =\phi(A \otimes y)=\mathcal{B}_{n}(\phi)(A)(y),
\end{aligned}
$$

pois $L(A \otimes y)=(A \otimes y)_{L}=A_{L} \otimes y$. Isso prova que $\mathcal{B}_{n}=J_{\ell} \circ \mathcal{B} \circ\left(L^{-1}\right)^{*}$ e completa a demonstração desta implicação.

(b) $\Longrightarrow$ (c) Essa implicação é óbvia.

(c) $\Longrightarrow$ (a) Suponha que $E^{*}$ tenha $P_{1}$ e $F$ tenha $P_{2}$. Cálculos rotineiros mostram que o operador

$$
v: E^{*} \longrightarrow \mathcal{L}(E, \mathbb{K}, \stackrel{(n-1)}{\cdots}, \mathbb{K}), v\left(x^{*}\right)\left(x, \lambda_{1}, \ldots, \lambda_{n-1}\right)=\lambda_{1} \cdots \lambda_{n-1} x^{*}(x),
$$

é um isomorfismo isométrico. Como $P_{1}$ é propriedade geométrica, $\mathcal{L}\left(E, \mathbb{K},{ }^{(n-1)}, \mathbb{K}\right)$ tem $P_{1}$, e daí a hiper-transformada de Borel $\mathcal{B}_{n}:[\mathcal{I} \circ \mathcal{L}(E, \mathbb{K}, \stackrel{(n-1)}{\cdots}, \mathbb{K} ; F)]^{*} \longrightarrow \mathcal{L}_{\alpha}(\mathcal{L}(E, \mathbb{K}, \stackrel{(n-1)}{\cdots}$ $\left., \mathbb{K}) ; F^{*}\right)$ é um isomorfismo isométrico por hipótese. Além disso, o operador de composição

$$
J_{v}: \mathcal{L}_{\alpha}\left(\mathcal{L}(E, \mathbb{K}, \stackrel{(n-1)}{\cdot}, \mathbb{K}) ; F^{*}\right) \longrightarrow \mathcal{L}_{\alpha}\left(E^{*} ; F^{*}\right), u \mapsto u \circ v
$$


é um isomorfismo isométrico pelo Lema 3.1.8. Conforme já usamos várias vezes, o operador linearização

$$
\ell: \mathcal{L}\left(E, \mathbb{K},{ }^{(n-1)}, \mathbb{K}\right) \longrightarrow\left(E \hat{\otimes}_{\pi} \mathbb{K} \hat{\otimes}_{\pi}{ }^{(n-1)} \hat{\otimes}_{\pi} \mathbb{K}\right)^{*}
$$

também é um isomorfismo isométrico. Assim a composta

$$
\ell \circ v: E^{*} \longrightarrow\left(E \hat{\otimes}_{\pi} \mathbb{K} \hat{\otimes}_{\pi}{ }^{(n-1)} \hat{\otimes}_{\pi} \mathbb{K}\right)^{*}
$$

também é. Seja $B_{L}: E \hat{\otimes}_{\pi} \mathbb{K} \hat{\otimes}_{\pi} \stackrel{(n-1)}{\cdots} \hat{\otimes}_{\pi} \mathbb{K} \longrightarrow E$ a linearização do operador multilinear contínuo

$$
B: E \times \mathbb{K}^{n-1} \longrightarrow E, B\left(x, \lambda_{1}, \ldots, \lambda_{n-1}\right)=\lambda_{1} \cdots \lambda_{n-1} x
$$

Para $x^{*} \in E^{*}$ e $x \otimes \lambda_{1} \otimes \cdots \otimes \lambda_{n-1} \in E \hat{\otimes}_{\pi} \mathbb{K} \hat{\otimes}_{\pi}{ }^{(n-1)} \hat{\otimes}_{\pi} \mathbb{K}$

$$
\begin{aligned}
B_{L}^{*}\left(x^{*}\right)\left(x \otimes \lambda_{1} \otimes \cdots \otimes \lambda_{n-1}\right) & =x^{*}\left(B\left(x, \lambda_{1}, \ldots, \lambda_{n-1}\right)\right) \\
& =\lambda_{1} \cdots \lambda_{n-1} x^{*}(x) \\
& =v\left(x^{*}\right)\left(x, \lambda_{1}, \ldots, \lambda_{n-1}\right) \\
& =v\left(x^{*}\right)_{L}\left(x \otimes \lambda_{1} \otimes \cdots \otimes \lambda_{n-1}\right) \\
& =(\ell \circ v)\left(x^{*}\right)\left(x \otimes \lambda_{1} \otimes \cdots \otimes \lambda_{n-1}\right) .
\end{aligned}
$$

Como ambos $B_{L}^{*}$ e $\ell \circ v$ são operadores lineares e contínuos e somas finitas de tensores elementares formam um conjunto denso em $E \hat{\otimes}_{\pi} \mathbb{K} \hat{\otimes}_{\pi}{ }^{(n-1)} \hat{\otimes}_{\pi} \mathbb{K}$, segue que $B_{L}^{*}=\ell \circ v$. Concluímos que $B_{L}$ é um isomorfismo isométrico.

Como $\mathcal{I}$ é um ideal de Banach de operadores, o operador de composição

$$
J_{\left(B_{L}\right)^{-1}}: \mathcal{I}\left(E \hat{\otimes}_{\pi} \mathbb{K} \hat{\otimes}_{\pi}{ }^{(n-1)} \hat{\otimes}_{\pi} \mathbb{K} ; F\right) \longrightarrow \mathcal{I}(E ; F), u \mapsto u \circ\left(B_{L}\right)^{-1}
$$

também é um isomorfismo isométrico (isso também segue do Lema 3.1.8). Usando as Proposições 1.4 .8 e 1.4 .9 novamente, o operador linearização

$$
L: \mathcal{I} \circ \mathcal{L}\left(E, \mathbb{K},{ }^{(n-1)}, \mathbb{K} ; F\right) \longrightarrow \mathcal{I}\left(E \hat{\otimes}_{\pi} \mathbb{K} \hat{\otimes}_{\pi}{ }^{(n-1)} \hat{\otimes}_{\pi} \mathbb{K} ; F\right)
$$

é um isomorfismo isométrico, assim como também o são o operador

$$
J_{\left(B_{L}\right)^{-1}} \circ L: \mathcal{I} \circ \mathcal{L}\left(E, \mathbb{K},{ }^{(n-1)}, \mathbb{K} ; F\right) \longrightarrow \mathcal{I}(E ; F)
$$

e seu adjunto

$$
\left(J_{\left(B_{L}\right)^{-1}} \circ L\right)^{*}:[\mathcal{I}(E ; F)]^{*} \longrightarrow[\mathcal{I} \circ \mathcal{L}(E, \mathbb{K}, \stackrel{(n-1)}{\cdots}, \mathbb{K} ; F)]^{*}
$$


Basta agora verificar que o diagrama

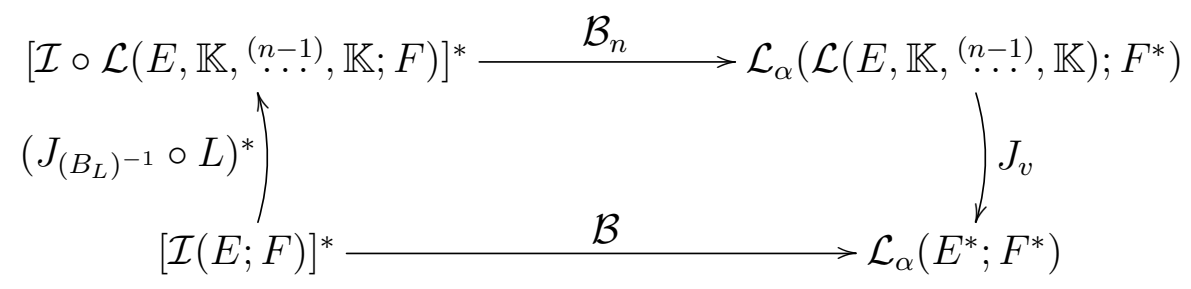

é comutativo. Primeiro, note que dados $x^{*} \in E^{*}$ e $y \in F$,

$$
\begin{aligned}
\left(\left(v\left(x^{*}\right)_{L} \otimes y\right) \circ\left(B_{L}\right)^{-1}\right)(x) & =\left(v\left(x^{*}\right)_{L} \otimes y\right)\left(\left(B_{L}\right)^{-1}(x)\right) \\
& =v\left(x^{*}\right)_{L}\left(\left(B_{L}\right)^{-1}(x)\right) y \\
& =v\left(x^{*}\right)_{L}(x \otimes 1 \otimes \cdots \otimes 1) y \\
& =v\left(x^{*}\right)(x, 1, \ldots, 1) y=x^{*}(x) y
\end{aligned}
$$

para todo $x \in E$, pois

$$
B_{L}(x \otimes 1 \otimes \cdots \otimes 1)=B(x, 1, \ldots, 1)=x .
$$

Isso prova que $\left(v\left(x^{*}\right)_{L} \otimes y\right) \circ\left(B_{L}\right)^{-1}=x^{*} \otimes y$. Segue que, para todos $\phi \in[\mathcal{I}(E ; F)]^{*}$, $x^{*} \in E^{*}$ e $y \in F$,

$$
\begin{aligned}
& \left(J_{v} \circ \mathcal{B}_{n} \circ\left(J_{\left(B_{L}\right)^{-1}} \circ L\right)^{*}\right)(\phi)\left(x^{*}\right)(y)=\left(J_{v}\left(\mathcal{B}_{n}\left(\left(J_{\left(B_{L}\right)^{-1}} \circ L\right)^{*}(\phi)\right)\right)\right)\left(x^{*}\right)(y) \\
& =\left(\left(\mathcal{B}_{n}\left(\left(J_{\left(B_{L}\right)^{-1}} \circ L\right)^{*}(\phi)\right)\right) \circ v\right)\left(x^{*}\right)(y)
\end{aligned}
$$

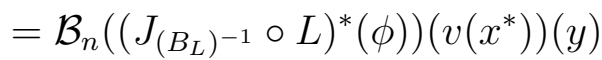

$$
\begin{aligned}
& =\left(J_{\left.\left(B_{L}\right)^{-1} \circ L\right)^{*}(\phi)\left(v\left(x^{*}\right) \otimes y\right)}\right. \\
& =\phi\left(\left(J_{\left(B_{L}\right)^{-1}} \circ L\right)\left(v\left(x^{*}\right) \otimes y\right)\right) \\
& =\phi\left(J_{\left(B_{L}\right)^{-1}}\left(L\left(v\left(x^{*}\right) \otimes y\right)\right)\right) \\
& =\phi\left(J_{\left(B_{L}\right)^{-1}}\left(\left(v\left(x^{*}\right) \otimes y\right)_{L}\right)\right) \\
& =\phi\left(J_{\left(B_{L}\right)^{-1}}\left(v\left(x^{*}\right)_{L} \otimes y\right)\right) \\
& =\phi\left(\left(v\left(x^{*}\right)_{L} \otimes y\right) \circ\left(B_{L}\right)^{-1}\right) \\
& =\phi\left(x^{*} \otimes y\right)=\mathcal{B}(\phi)\left(x^{*}\right)(y) \text {. }
\end{aligned}
$$

Isso prova que $\mathcal{B}=J_{v}\left(\mathcal{B}_{n}\left(\left(J_{\left(B_{L}\right)^{-1}} \circ L\right)^{*}\right)\right)$ e completa a demonstração.

Observação 3.2.2. Da demonstração acima segue que se $\left(\mathcal{I},\|\cdot\|_{\mathcal{I}}\right)$ é um ideal de Banach, $\alpha$ um semi-ideal à esquerda, $E_{1}, \ldots, E_{n}$ espaços de Banach e a transformada de Borel linear $\mathcal{B}:\left(\mathcal{I}\left(E_{1} \hat{\otimes}_{\pi} \cdots \hat{\otimes}_{\pi} E_{n} ; F\right),\|\cdot\|_{\mathcal{I}}\right)^{*} \longrightarrow \mathcal{L}_{\alpha}\left(\left(E_{1} \hat{\otimes}_{\pi} \cdots \hat{\otimes}_{\pi} E_{n}\right)^{*} ; F^{*}\right), \mathcal{B}(\phi)\left(x^{*}\right)(y)=\phi\left(x^{*} \otimes y\right)$ 
é um isomorfismo isométrico, então a hiper-transformada de Borel $\mathcal{B}_{n}:\left(\mathcal{I} \circ \mathcal{L}\left(E_{1}, \ldots, E_{n} ; F\right),\|\cdot\|_{\mathcal{I} \circ \mathcal{L}}\right)^{*} \longrightarrow \mathcal{L}_{\alpha}\left(\mathcal{L}\left(E_{1}, \ldots, E_{n}\right) ; F^{*}\right), \mathcal{B}_{n}(\phi)(A)(y)=\phi(A \otimes y)$, é um isomorfismo isométrico.

\subsection{Aplicações}

Nesta seção daremos algumas aplicações concretas do Teorema 3.2.1, isto é, obteremos representações de duais em classes concretas de operadores multilineares por meio da hiper-transformada de Borel.

\subsubsection{Operadores multilineares aproximáveis e compactos}

Relembre (veja Definição 1.3.2) que $\overline{\mathcal{F}}$ representa o fecho do ideal dos operadores lineares de posto finito, isto é, são os operadores que podem ser aproximados, na norma usual, por operadores de posto finito. E que $\overline{\mathcal{L}_{\mathcal{F}}}$ representa o fecho do multi-ideal dos operadores multilineares de posto finito, isto é, são os operadores multilineares que podem ser aproximados, na norma usual, por operadores multilineares de posto finito.

Relembremos a definição do ideal de Banach dos operadores integrais. Um operador $u: E \longrightarrow F$ é integral se existem um espaço de medida finita $(\Omega, \Sigma, \mu)$ e operadores $S: E \longrightarrow L_{\infty}(\mu), R: L_{1}(\mu) \longrightarrow F^{* *}$ tais que $J_{F} \circ u=R \circ I \circ S$, onde $I: L_{\infty}(\mu) \longrightarrow L_{1}(\mu)$ é o operador canônico (inclusão). Neste caso escrevemos $u \in \mathcal{J}(E ; F)$ e definimos a norma integral por

$$
\|u\|_{\mathcal{J}}=\inf \{\|S\| \cdot\|R\| \cdot \mu(\Omega)\}
$$

onde o ínfimo é tomado sobre todas essas fatorações de $J_{F} \circ u$. É conhecido que $\left(\mathcal{J},\|\cdot\|_{\mathcal{J}}\right)$ é um ideal de Banach. Para maiores informações veja, por exemplo, [67, Seção 3.5]. Espaços de operadores integrais serão sempre considerados munidos da norma integral.

Por $E \widehat{\otimes}_{\varepsilon} F$ denotamos o produto tensorial injetivo (completado) dos espaços de Banach $E$ e $F$. Como usaremos esse espaço apenas pontualmente, omitimos a definição e remetemos o leitor para os livros de Ryan [67, Capítulo 3] e de Defant e Floret [33, Seção I.4].

Teorema 3.3.1. Para todos espaços de Banach $E_{1}, \ldots, E_{n}$ e F, a hiper-transformada de Borel

$$
\mathcal{B}_{n}:\left[\overline{\mathcal{L}_{\mathcal{F}}}\left(E_{1}, \ldots, E_{n} ; F\right)\right]^{*} \longrightarrow \mathcal{J}\left(\mathcal{L}\left(E_{1}, \ldots, E_{n}\right) ; F^{*}\right), \mathcal{B}_{n}(\phi)(B)(y)=\phi(B \otimes y),
$$


é um isomorfismo isométrico.

Demonstração. É conhecido que o operador

$$
T: E^{*} \widehat{\otimes}_{\varepsilon} F \longrightarrow \overline{\mathcal{F}}(E ; F), T\left(x^{*} \otimes y\right)(x)=x^{*}(x) y
$$

é um isomorfismo isométrico (veja [33, 4.2.(1)]), e portanto também o é o seu adjunto

$$
T^{*}:[\overline{\mathcal{F}}(E ; F)]^{*} \longrightarrow\left(E^{*} \widehat{\otimes}_{\varepsilon} F\right)^{*} .
$$

E é também conhecido que o operador

$$
U:\left(E^{*} \widehat{\otimes}_{\varepsilon} F\right)^{*} \longrightarrow \mathcal{J}\left(E^{*}, F^{*}\right), U(\psi)\left(x^{*}\right)(y)=\psi\left(x^{*} \otimes y\right),
$$

é um isomorfismo isométrico (veja $[33,10.1]$ ). Vejamos que $\mathcal{B}=U \circ T^{*}$ : para todos $\phi \in[\overline{\mathcal{F}}(E ; F)]^{*}, x^{*} \in E^{*}$ e $y \in F$,

$\left(U \circ T^{*}\right)(\phi)\left(x^{*}\right)(y)=U\left(T^{*}(\phi)\right)\left(x^{*}\right)(y)=T^{*}(\phi)\left(x^{*} \otimes y\right)=\phi\left(T\left(x^{*} \otimes y\right)\right)=\phi\left(x^{*} \otimes y\right)=\mathcal{B}(\phi)\left(x^{*}\right)(y)$.

Isso mostra que a transformada de Borel linear $\mathcal{B}:[\overline{\mathcal{F}}(E ; F)]^{*} \longrightarrow \mathcal{J}\left(E^{*}, F^{*}\right)$ é um isomorfismo isométrico para todos espaços de Banach $E$ e $F$.

Como $\mathcal{J}$ é um ideal de Banach, em particular é um semi-ideal à esquerda, do Teorema 3.2.1 segue que a hiper-transformada de Borel

$$
\mathcal{B}_{n}:\left[\overline{\mathcal{F}} \circ \mathcal{L}\left(E_{1}, \ldots, E_{n} ; F\right)\right]^{*} \longrightarrow \mathcal{J}\left(\mathcal{L}\left(E_{1}, \ldots, E_{n}\right) ; F^{*}\right)
$$

é um isomorfismo isométrico para todos espaços de Banach $E_{1}, \ldots, E_{n}$ e $F$. Pela Proposição 1.4.10(b) sabemos que $\overline{\mathcal{L}_{\mathcal{F}}}\left(E_{1}, \ldots, E_{n} ; F\right)=\overline{\mathcal{F}} \circ \mathcal{L}\left(E_{1}, \ldots, E_{n} ; F\right)$ isometricamente, o que conclui a demonstração.

Seja $\mathcal{L}_{\mathcal{J}}$ a classe dos operadores multilineares integrais (veja [67]). Vale notar que em Alencar [4] está provado que

$$
\left[\overline{\mathcal{L}_{f}}\left(E_{1}, \ldots, E_{n} ; F\right)\right]^{*}=\mathcal{L}_{\mathcal{J}}\left(E_{1}^{*}, \ldots, E_{n}^{*} ; F^{*}\right)
$$

por meio da transformada de Borel, se $E_{1}, \ldots, E_{n}$ e $F$ são reflexivos. Além de representarmos funcionais lineares como operadores lineares ao invés de operadores multilineares, nossa representação no Teorema 3.3.1 funciona para quaisquer espaços de Banach. Isso mostra que, pelo menos em algumas vezes, as representações obtidas pela hipertransformada de Borel são mais gerais que as representações correspondentes obtidas pela transformada de Borel. 
$\mathrm{Na}$ aplicação seguinte, sob a presença da propriedade da aproximação, usaremos o Teorema 3.2.1 para representar funcionais lineares em espaços de operadores multilineares compactos como operadores lineares integrais. Tanto quanto sabemos, não há na literatura nenhuma outra representação de funcionais lineares em espaços de operadores multilineares compactos. Relembre que por $\mathcal{L}_{\mathcal{K}}$ denotamos o hiper-ideal dos operadores multilineares compactos.

Teorema 3.3.2. Sejam $E_{1}, \ldots, E_{n}, F$ espaços de Banach tais que $\mathcal{L}\left(E_{1}, \ldots, E_{n}\right)$ ou $F$ tem a propriedade da aproximação. Então a hiper-transformada de Borel

$$
\mathcal{B}_{n}:\left[\mathcal{L}_{\mathcal{K}}\left(E_{1}, \ldots, E_{n} ; F\right)\right]^{*} \longrightarrow \mathcal{J}\left(\mathcal{L}\left(E_{1}, \ldots, E_{n}\right) ; F^{*}\right), \mathcal{B}_{n}(\phi)(B)(y)=\phi(B \otimes y)
$$

é um isomorfismo isométrico.

Demonstração. Supondo que $E^{*}$ ou $F$ possuam a propriedade da aproximação, da Proposição 1.1 .10 sabemos que

$$
\overline{\mathcal{F}}(E ; F)=\mathcal{K}(E ; F) .
$$

Da primeira parte da demonstração do teorema anterior, segue que a transformada de Borel linear

$$
\mathcal{B}:[\mathcal{K}(E ; F)]^{*} \longrightarrow \mathcal{J}\left(E^{*} ; F^{*}\right), \mathcal{B}(\phi)(\varphi)(y)=\phi(\varphi \otimes y),
$$

é um isomorfismo isométrico desde que $E^{*}$ ou $F$ tenha a propriedade da aproximação. Como $\mathcal{J}$ é um semi-ideal à esquerda, pois é um ideal de Banach, concluímos pelo Teorema 3.2.1 que a hiper-transformada de Borel

$$
\mathcal{B}_{n}:\left[\mathcal{K} \circ \mathcal{L}\left(E_{1}, \ldots, E_{n} ; F\right)\right]^{*} \longrightarrow \mathcal{J}\left(\mathcal{L}\left(E_{1}, \ldots, E_{n}\right) ; F^{*}\right), \mathcal{B}_{n}(\phi)(B)(y)=\phi(B \otimes y)
$$

é um isomorfismo isométrico se $\mathcal{L}\left(E_{1}, \ldots, E_{n}\right)$ ou $F$ tem a propriedade da aproximação. Pela Proposição 1.4.10(a) sabemos $\left.\mathcal{L}_{\mathcal{K}} E_{1}, \ldots, E_{n} ; F\right)=\mathcal{K} \circ \mathcal{L}\left(E_{1}, \ldots, E_{n} ; F\right)$ isometricamente para todos espaços de Banach $E_{1}, \ldots, E_{n}$ e $F$, o que conclui a demonstração.

\subsubsection{Operadores multilineares hiper-nucleares e hiper- $(s ; r)$ - nucleares}

Conforme anunciado após o Teorema 3.1.3, provaremos agora que a hiper-transformada de Borel representa funcionais lineares em espaços de operadores hiper-nucleares como operadores lineares com a norma usual de operadores. 
Acreditamos que o caso linear do resultado que queremos provar seja conhecido, mas como não encontramos nenhuma referência com demonstração, apresentaremos um argumento rápido. Mais à frente mencionaremos o caso multilinear desse resultado, mas que não contempla o caso em que apenas $F$ tem a propriedade da aproximação limitada.

Proposição 3.3.3. Se $E^{*}$ ou $F$ tem a propriedade da aproximação limitada, então a transformada de Borel linear

$$
\mathcal{B}:[\mathcal{N}(E ; F)]^{*} \longrightarrow \mathcal{L}\left(E^{*} ; F^{*}\right), \mathcal{B}(\phi)(\varphi)(y)=\phi(\varphi \otimes y)
$$

é um isomorfismo isométrico.

Demonstração. Pela Proposição 1.3.4, sabemos que existe um operador

$$
T: E^{*} \widehat{\otimes}_{\pi} F \longrightarrow \mathcal{N}(E ; F), T\left(x^{*} \otimes y\right)(x)=x^{*}(x) y
$$

que é um isomorfismo isométrico, e assim também o é o seu adjunto

$$
T^{*}:[\mathcal{N}(E ; F)]^{*} \longrightarrow\left(E^{*} \widehat{\otimes}_{\pi} F\right)^{*}
$$

Das Observações 1.2.7 e 1.2.14, sabemos que os operadores

$$
\begin{gathered}
U:\left(E^{*} \widehat{\otimes}_{\pi} F\right)^{*} \longrightarrow \mathcal{L}\left(E^{*}, F ; \mathbb{K}\right), U(\psi)\left(x^{*}, y\right)=\psi\left(x^{*} \otimes y\right), \\
V: \mathcal{L}\left(E^{*}, F ; \mathbb{K}\right) \longrightarrow \mathcal{L}\left(E^{*} ; F^{*}\right), V(A)\left(x^{*}\right)(y)=A\left(x^{*}, y\right),
\end{gathered}
$$

são isomorfismos isométricos. É suficiente mostrar que $\mathcal{B}=V \circ U \circ T^{*}$. De fato, para todos $\phi \in[\mathcal{N}(E ; F)]^{*}, x^{*} \in E^{*}$ e $y \in F$,

$$
\begin{aligned}
\left(V \circ U \circ T^{*}\right)(\phi)\left(x^{*}\right)(y) & =V\left(U\left(T^{*}(\phi)\right)\right)\left(x^{*}\right)(y) \\
& =U\left(T^{*}(\phi)\right)\left(x^{*}, y\right) \\
& =T^{*}(\phi)\left(x^{*} \otimes y\right) \\
& =\phi\left(T\left(x^{*} \otimes y\right)\right) \\
& =\mathcal{B}(\phi)\left(x^{*}\right)(y) .
\end{aligned}
$$

Agora sim, podemos provar a representação desejada. 
Teorema 3.3.4. Se $\mathcal{L}\left(E_{1}, \ldots, E_{n}\right)$ ou $F$ tem a propriedade da aproximação limitada, então a hiper-transformada de Borel

$$
\mathcal{B}_{n}:\left[\mathcal{L}_{\mathcal{H N}}\left(E_{1}, \ldots, E_{n} ; F\right)\right]^{*} \longrightarrow \mathcal{L}\left(\mathcal{L}\left(E_{1}, \ldots, E_{n}\right) ; F^{*}\right), \mathcal{B}_{n}(\phi)(B)(y)=\phi(B \otimes y),
$$

é um isomorfismo isométrico.

Demonstração. Combinando a representação linear da Proposição 3.3 .3 com o Teorema 3.2.1, obtemos que a hiper-transformada de Borel

$$
\mathcal{B}_{n}:\left[\mathcal{N} \circ \mathcal{L}\left(E_{1}, \ldots, E_{n} ; F\right)\right]^{*} \longrightarrow \mathcal{L}\left(\mathcal{L}\left(E_{1}, \ldots, E_{n}\right) ; F^{*}\right), \mathcal{B}_{n}(\phi)(B)(y)=\phi(B \otimes y),
$$

é um isomorfismo isométrico. Finalmente, do Corolário 2.1.6 sabemos que $\mathcal{L}_{\mathcal{H N}}=\mathcal{N} \circ \mathcal{L}$ isometricamente, e portanto a demonstração está completa.

Para cotejar o teorema acima com o que já é conhecido, mencionamos que, melhorando um resultado anterior devido a Gupta [46], Carando e Dimant provaram em [31] que

$$
\left[\mathcal{L}_{\mathcal{N}}\left(E_{1}, \ldots, E_{n} ; F\right)\right]^{*}=\mathcal{L}\left(E_{1}^{*}, \ldots, E_{n}^{*} ; F^{*}\right)
$$

por meio da transformada de Borel multilinear, desde que $E_{1}^{*}, \ldots, E_{n}^{*}$ tenham a propriedade da aproximação. Além de representar os funcionais lineares como operadores lineares ao invés de operadores multilineares, a representação do Teorema 3.3.4 vale para todos espaços de Banach $E_{1}, \ldots, E_{n}$ desde que $F$ tenha a propriedade da aproximação limitada.

$\mathrm{Na}$ aplicação a seguir, usaremos o Teorema 3.2.1 para representar funcionais lineares em espaços de operadores hiper- $(s ; r)$-nucleares como operadores lineares absolutamente somantes (Exemplo 1.4.5).

Teorema 3.3.5. Sejam $1 \leq s, r<\infty$ tais que $1 \leq \frac{1}{s}+\frac{1}{r}$ e suponha que $\mathcal{L}\left(E_{1}, \ldots, E_{n}\right)$ tenha a propriedade da aproximação limitada. Então, para todo espaço de Banach $F$, a hiper-transformada de Borel

$$
\mathcal{B}_{n}:\left[\mathcal{L}_{\mathcal{H N}}\left(E_{(s ; r)}\left(E_{1}, \ldots, E_{n} ; F\right)\right]^{*} \longrightarrow \prod_{s^{*}, r}\left(\mathcal{L}\left(E_{1}, \ldots, E_{n}\right) ; F^{*}\right)\right.
$$

é um isomorfismo isométrico.

Demonstração. O caso linear do Exemplo 3.0.2 afirma que se $E^{*}$ tem a propriedade da aproximação limitada, então a transformada de Borel linear

$$
\mathcal{B}:\left[\mathcal{N}_{(s ; r)}(E ; F)\right]^{*} \longrightarrow \Pi_{s^{*}, r}\left(E^{*} ; F^{*}\right)
$$

é um isomorfismo isométrico. Como $\Pi_{\left(s^{*} ; r\right)}$ é um ideal de Banach [34, Proposition 10.2], e portanto um semi-ideal à esquerda, combinando o Teorema 3.2.1 com a Proposição 2.1.5 obtemos a representação desejada. 


\subsubsection{Operadores multilineares hiper- $\sigma(p)$-nucleares}

Até o momento apresentamos aplicações que envolviam representações de funcionais lineares em classes de operadores multilineares já estudadas. Nesta subseção utilizaremos o Teorema 3.2.1 para representar funcionais lineares em espaços de operadores multilineares hiper- $\sigma(p)$-nucleares, que foram por nós introduzidos na Seção 2.2.

Essa representação tem um significado importante, pois representaremos tais funcionais como operadores lineares quase- $\tau(p)$-somantes, os quais, conforme vimos no Exemplo 3.1.6, formam um semi-ideal à esquerda que não é um ideal de operadores. Este é um caso concreto em que a generalidade do Teorema 3.2.1 conferida pela noção de semi-ideal à esquerda, por nós introduzida, é necessária. Se tivéssemos provado o teorema com ideais de operadores, a representação a seguir não seria possível.

Teorema 3.3.6. Seja $1 \leq p<\infty$ e suponhamos que o espaço $\mathcal{L}\left(E_{1}, \ldots, E_{n}\right)$ possua a propriedade da aproximação limitada. Então, para todo espaço de Banach $F$, a hipertransformada de Borel

$$
\mathcal{B}_{n}:\left[\mathcal{L}_{\mathcal{H} \sigma(p)}\left(E_{1}, \ldots, E_{n} ; F\right)\right]^{*} \longrightarrow \mathcal{L}_{q \tau(p)}\left(\mathcal{L}\left(E_{1}, \ldots, E_{n}\right) ; F^{*}\right), \mathcal{B}_{n}(\phi)(B)(y)=\phi(B \otimes y),
$$

é um isomorfismo isométrico.

Demonstração. Do Exemplo 3.0.4 sabemos que a transformada de Borel linear

$$
\mathcal{B}:\left[\mathcal{N}_{\sigma(p)}(E ; F)\right]^{*} \longrightarrow \mathcal{L}_{q \tau(p)}\left(E^{*} ; F^{*}\right), \mathcal{B}(\phi)(\varphi)(y)=\phi(\varphi \otimes y),
$$

é um isomorfismo isométrico desde que $E^{*}$ tenha a propriedade da aproximação limitada. Como $\mathcal{L}_{q \tau(p)}$ é um semi-ideal à esquerda (Exemplo 3.1.6), o resultado segue combinando o Teorema 3.2.1 com o Teorema 2.2.6. 


\section{Capítulo 4}

\section{O Caso Polinomial}

Neste capítulo desenvolveremos a contrapartida polinomial da teoria desenvolvida nos Capítulos 2 e 3 para operadores multilineares. Invertendo um pouco a ordem, trataremos primeiro da hiper-transformada de Borel para polinômios homogêneos e depois estudaremos os polinômios hiper- $\sigma(p)$-nucleares nas aplicações da hiper-transformada de Borel.

Demonstrações e mais detalhes da teoria de hiper-ideais de polinômios homogêneos podem ser encontrados em [21, 72].

\subsection{A hiper-transformada de Borel polinomial}

Nesta seção desenvolveremos uma teoria análoga à do capítulo anterior para classes de polinômios homogêneos. Como é bem sabido, muitas definições e resultados, no contexto polinomial, funcionam paralelamente ao caso multilinear, mas as duas teorias não são idênticas, pois no caso polinomial há menos margem de manobra. Por exemplo, argumentos multilineares em que uma variável é fixada - ou várias variáveis são fixadas enquanto as demais variam, não se aplicam ao caso polinomial. Nesta seção veremos os dois fenômenos acontecerem: em algumas situações o caso polinomial flui como no caso multilinear, e em outras o caso polinomial se mostra mais difícil que o caso multilinear. Em particular, uma diferença inesperada entre as teorias multilinear e polinomial será revelada na Seção 4.2 .

A transformada de Borel para espaços de polinômios homogêneos é o operador

$$
\beta_{n}:\left(\mathcal{Q}\left({ }^{n} E ; F\right),\|\cdot\|_{\mathcal{Q}}\right)^{*} \longrightarrow \mathcal{P}\left({ }^{n} E^{*} ; F^{*}\right), \beta_{n}(\phi)\left(x^{*}\right)(y)=\phi\left(\left(x^{*}\right)^{n} \otimes y\right),
$$


onde $\mathcal{Q}\left({ }^{n} E ; F\right)$ é um subespaço de $\mathcal{P}\left({ }^{n} E ; F\right)$ munido de uma norma completa $\|\cdot\|_{\mathcal{Q}}$ e contendo os polinômios de tipo finito. Muitas aplicações dessa transformada podem ser encontradas na literatura, por exemplo [4, 25, 31, 35, 37, 38, 39, 40, 41, 47, 52, 58].

Assim como no caso multilinear, para que $\beta_{n}$ seja um isomorfismo isométrico sobre um subespaço de $\mathcal{P}\left({ }^{n} E^{*} ; F^{*}\right)$, munido com uma norma completa, é necessário que os

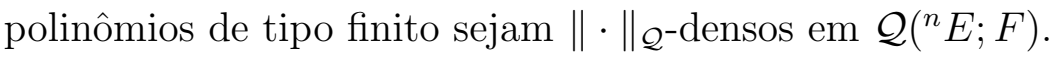

Nesta seção introduziremos a hiper-transformada de Borel polinomial, que, conforme veremos na seção seguinte, permitirá a representação de duais em espaços de polinômios que não são alcançados pela transformada de Borel polinomial.

Proposição 4.1.1. Seja $\mathcal{Q}\left({ }^{n} E ; F\right)$ um subespaço vetorial de $\mathcal{P}\left({ }^{n} E ; F\right)$ munido de uma norma completa $\|\cdot\|_{\mathcal{Q}}$ contendo os polinômios n-homogêneos de posto finito $\mathcal{P}_{\mathcal{F}}\left({ }^{n} E ; F\right) e$ tal que $\|P \otimes y\|_{\mathcal{Q}} \leq\|P\| \cdot\|y\|$, para todos $P \in \mathcal{P}\left({ }^{n} E\right)$ e $y \in F$. Então:

(a) A aplicação

$$
\mathfrak{B}_{n}:\left(\mathcal{Q}\left({ }^{n} E ; F\right),\|\cdot\|_{\mathcal{Q}}\right)^{*} \longrightarrow \mathcal{L}\left(\mathcal{P}\left({ }^{n} E\right) ; F^{*}\right), \mathfrak{B}_{n}(\phi)(P)(y)=\phi(P \otimes y),
$$

é um operador linear bem definido e $\left\|\mathfrak{B}_{n}\right\| \leq 1$.

(b) $\mathfrak{B}_{n}$ é injetiva se, e somente se, o subespaço dos polinômios n-homogêneos de posto

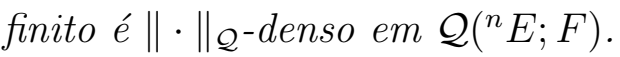

Demonstração. (a) Vejamos que $\mathfrak{B}_{n}$ está bem definida. Para $P \in \mathcal{P}\left({ }^{n} E\right)$ e $y \in F$, $P \otimes y \in \mathcal{P}_{\mathcal{F}}\left({ }^{n} E ; F\right) \subseteq \mathcal{Q}\left({ }^{n} E ; F\right)$, e portanto podemos calcular $\mathfrak{B}_{n}(\phi)(P)(y)$ para todo funcional $\phi \in\left[\mathcal{Q}\left({ }^{n} E ; F\right)\right]^{*}$. A linearidade de $\mathfrak{B}_{n}(\phi)(P)$ é imediata e sua continuidade segue de

$$
\left|\mathfrak{B}_{n}(\phi)(P)(y)\right|=|\phi(P \otimes y)| \leq\|\phi\|_{\mathcal{Q}^{*}} \cdot\|P \otimes y\|_{\mathcal{Q}} \leq\|\phi\|_{\mathcal{Q}^{*}} \cdot\|P\| \cdot\|y\|
$$

para todo $y \in F$. Isso prova que $\mathfrak{B}_{n}(\phi)(P) \in F^{*}$. A linearidade de $\mathfrak{B}_{n}(\phi): \mathcal{P}\left({ }^{n} E\right) \longrightarrow F^{*}$ também é facilmente verificada e de

$$
\begin{aligned}
\left\|\mathfrak{B}_{n}(\phi)(P)\right\| & =\sup _{y \in B_{F}}\left|\mathfrak{B}_{n}(\phi)(P)(y)\right|=\sup _{y \in B_{F}}|\phi(P \otimes y)| \\
& \leq \sup _{y \in B_{F}}\|\phi\|_{\mathcal{Q}^{*}} \cdot\|P \otimes y\|_{\mathcal{Q}}=\|\phi\|_{\mathcal{Q}^{*}} \cdot \sup _{y \in B_{F}}\|P \otimes y\|_{\mathcal{Q}} \\
& \leq\|\phi\|_{\mathcal{Q}^{*}} \cdot \sup _{y \in B_{F}}\|P\| \cdot\|y\|=\|\phi\|_{\mathcal{Q}^{*}} \cdot\|P\|
\end{aligned}
$$

para todo $P \in \mathcal{P}\left({ }^{n} E\right)$, obtemos sua continuidade. Isso prova que $\mathfrak{B}_{n}(\phi) \in \mathcal{L}\left(\mathcal{P}\left({ }^{n} E\right) ; F^{*}\right)$. Mais uma vez, a linearidade de $\mathfrak{B}_{n}$ é clara, e de

$$
\left\|\mathfrak{B}_{n}(\phi)\right\|=\sup _{P \in B_{\mathcal{P}\left({ }^{n} E\right)}}\left\|\mathfrak{B}_{n}(\phi)(P)\right\|
$$




$$
\begin{aligned}
& =\sup _{P \in B_{\mathcal{P}\left({ }^{n} E\right)}, y \in B_{F}}\left|\mathfrak{B}_{n}(\phi)(P)(y)\right|=\sup _{P \in B_{\mathcal{P}\left(n_{E}\right)}, y \in B_{F}}|\phi(P \otimes y)| \\
& \leq \sup _{P \in B_{\mathcal{P}\left({ }^{n} E\right)}, y \in B_{F}}\|\phi\|_{\mathcal{Q}^{*}} \cdot\|P \otimes y\|_{\mathcal{Q}} \\
& =\|\phi\|_{\mathcal{Q}^{*}} \cdot \sup _{P \in B_{\mathcal{P}\left({ }^{n}\right)}, y \in B_{F}}\|P \otimes y\|_{\mathcal{Q}} \\
& \leq\|\phi\|_{\mathcal{Q}^{*}} \cdot \sup _{P \in B_{\mathcal{P}\left({ }^{n} E\right)}, y \in B_{F}}\|P\| \cdot\|y\|=\|\phi\|_{\mathcal{Q}^{*}}
\end{aligned}
$$

para todo $\phi \in\left[\mathcal{Q}\left({ }^{n} E ; F\right)\right]^{*}$, segue sua continuidade e também que $\left\|\mathfrak{B}_{n}\right\| \leq 1$.

(b) Suponhamos que os polinômios de posto finito sejam $\|\cdot\|_{\mathcal{Q}^{-}}$densos em $\mathcal{Q}\left({ }^{n} E ; F\right)$ e seja $\phi \in \operatorname{ker}\left(\mathfrak{B}_{n}\right)$. Então, para $P=\sum_{j=1}^{k} P_{j} \otimes y_{j} \in \mathcal{P}_{\mathcal{F}}\left({ }^{n} E ; F\right)$,

$$
\phi\left(\sum_{j=1}^{k} P_{j} \otimes y_{j}\right)=\sum_{j=1}^{k} \phi\left(P_{j} \otimes y_{j}\right)=\sum_{j=1}^{k} \mathfrak{B}_{n}(\phi)\left(P_{j}\right)\left(y_{j}\right)=0 .
$$

Logo, $\phi$ se anula no subespaço denso dos polinômios de posto finito. Como $\phi$ é contínua, segue que $\phi=0, \log _{0} \mathfrak{B}_{n}$ é injetora.

Reciprocamente, suponhamos que $\mathfrak{B}_{n}$ seja injetora. Tome $\phi \in\left[\mathcal{Q}\left({ }^{n} E ; F\right)\right]^{*}$ tal que $\phi(P)=0$ para todo $P \in \mathcal{P}_{\mathcal{F}}\left({ }^{n} E ; F\right)$. Então,

$$
\mathfrak{B}_{n}(\phi)(Q)(y)=\phi(Q \otimes y)=0
$$

para todos $Q \in \mathcal{P}\left({ }^{n} E\right)$ e $y \in F$, e portanto $\mathfrak{B}_{n}(\phi)=0$. Segue da injetividade de $\mathfrak{B}_{n}$ que $\phi=0$. Pela Proposição 1.1.8 obtemos que o subespaço dos polinômios de posto finito é $\|\cdot\|_{\mathcal{Q}^{-}}$denso em $\mathcal{Q}\left({ }^{n} E ; F\right)$.

O operador $\mathfrak{B}_{n}$ é chamado de hiper-transformada de Borel polinomial. Como já fizemos para operadores multilineares, no caso linear $n=1$ o operador $\mathfrak{B}_{1}$ coincide com a transformada de Borel linear $\beta$. Escreveremos simplesmente $\mathfrak{B}$ nesse caso.

Como os ideais de polinômios contêm os polinômios de tipo finito, a transformada de Borel polinomial é, em geral, aplicada a ideais de polinômios. Já a hiper-transformada de Borel exige que a classe contenha os polinômios de posto finito. Os hiper-ideais de polinômios, introduzidos em [72] (veja também [21]) e definidos a seguir, satisfazem essa propriedade, e por isso aplicaremos a hiper-transformada de Borel para representação de duais em hiper-ideais de polinômios. 
Definição 4.1.2. Sejam $\mathcal{Q}$ uma classe de polinômios homogêneos entre espaços de Banach, $\|\cdot\|_{\mathcal{Q}}: \mathcal{Q} \longrightarrow \mathbb{R}$ uma função e $\left(C_{n}\right)_{n=1}^{\infty}$ uma sequência de números reais positivos com $C_{n} \geq 1$ para todo $n \in \mathbb{N}$ e $C_{1}=1$. Para todo $n \in \mathbb{N}$ e todos espaços de Banach $E$ e $F$, suponha que:

(i) A componente

$$
\mathcal{Q}\left({ }^{n} E ; F\right):=\mathcal{P}\left({ }^{n} E ; F\right) \cap \mathcal{Q}
$$

é um subespaço vetorial de $\mathcal{P}\left({ }^{n} E ; F\right)$ contendo os polinômios $n$-homogêneos de tipo finito.

(ii) A restrição de $\|\cdot\|_{\mathcal{Q}}$ a $\mathcal{Q}\left({ }^{n} E ; F\right)$ é uma norma.

(iii) $\left\|\hat{I}_{n}: \mathbb{K} \longrightarrow \mathbb{K}, \hat{I}_{n}(\lambda)=\lambda^{n}\right\|_{\mathcal{Q}}=1$ para todo $n$.

Dizemos que $\left(\mathcal{Q},\|\cdot\|_{\mathcal{Q}}\right)$ é um $\left(C_{n}\right)_{n=1}^{\infty}$-hiper-ideal normado de polinômios se satisfaz a propriedade de hiper-ideal: Para $n, m \in \mathbb{N}$, e espaços de Banach $E, F, G$ e $H$, se $P \in \mathcal{Q}\left({ }^{n} E ; F\right), Q \in \mathcal{P}\left({ }^{m} G ; E\right)$ e $t \in \mathcal{L}(F ; H)$, então $t \circ P \circ Q \in \mathcal{Q}\left({ }^{m n} G ; H\right)$ e

$$
\|t \circ P \circ Q\|_{\mathcal{Q}} \leq C_{m}^{m} \cdot\|t\| \cdot\|P\|_{\mathcal{Q}} \cdot\|Q\|^{n} .
$$

Quando $C_{n}=1$ para todo $n \in \mathbb{N}$, dizemos simplesmente que $\left(\mathcal{Q},\|\cdot\|_{\mathcal{Q}}\right)$ é um hiperideal normado de polinômios. Se as componentes $\mathcal{Q}\left({ }^{n} E ; F\right)$ são completas com respeito à topologia gerada por $\|\cdot\|_{\mathcal{Q}}$, então $\left(\mathcal{Q},\|\cdot\|_{\mathcal{Q}}\right)$ é chamado de $\left(C_{n}\right)_{n=1}^{\infty}$-hiper-ideal de Banach de polinômios.

Na presença da propriedade de hiper-ideal, conter os polinômios de tipo finito é equivalente a conter os polinômios de posto finito.

Quando a propriedade de hiper-ideal vale para todo $n \in \mathbb{N}$, mas somente para $m=1$, recupera-se o conceito de ideal normado de polinômios.

Exemplo 4.1.3. Seja $\left(\mathcal{Q},\|\cdot\|_{\mathcal{Q}}\right)$ um hiper-ideal de Banach de polinômios. Então, $\left(\mathcal{Q}\left({ }^{n} E ; F\right),\|\cdot\|_{\mathcal{Q}}\right)$ satisfaz as condições da Proposição $4.1 .1 \mathrm{com}\|Q \otimes y\|_{\mathcal{Q}}=\|Q\| \cdot\|y\|$ para todos $n \in \mathbb{N}, P \in \mathcal{P}\left({ }^{n} E\right)$ e $y \in F$ (veja [21, Proposition 2.3]). Isso faz com que os hiper-ideais de Banach de polinômios sejam os objetos adequados para se aplicar a hiper-transformada de Borel.

No teorema a seguir vemos que os ideais de polinômios de composição são exemplos de hiper-ideais de polinômios.

Teorema 4.1.4. [72, Teorema 3.4.5] Se $\mathcal{I}$ é um ideal de operadores, então $\mathcal{I} \circ \mathcal{P}$ é um hiper-ideal de polinômios. Além disso, se $\left(\mathcal{I},\|\cdot\|_{\mathcal{I}}\right)$ for ideal normado (respectivamente Banach) de operadores, então $\left(\mathcal{I} \circ \mathcal{P},\|\cdot\|_{\mathcal{I} \circ \mathcal{P})}\right.$ é hiper-ideal normado (respectivamente Banach) de polinômios. 
Tendo em vista o caso multilinear, para investigar quando a hiper-transformada de Borel é um isomorfismo sobre um subespaço de $\mathcal{L}\left(\mathcal{P}\left({ }^{n} E\right) ; F^{*}\right)$, devemos focar nas classes de polinômios do tipo nucleares. Os polinômios homogêneos nucleares foram introduzidos por Gupta [46, 47], generalizados por Matos [52] e posteriormente estudadas por vários autores, incluindo as referências sobre dinâmica linear citadas anteriormente. Para o nosso estudo, serão úteis as seguintes classes de polinômios nucleares introduzidas em [72].

Definição 4.1.5. [72] Sejam $s \in[1, \infty)$ e $r \in[1, \infty]$ tais que $1 \leq \frac{1}{s}+\frac{1}{r}$. Um polinômio $n$-homogêneo $P \in \mathcal{P}\left({ }^{n} E ; F\right)$ é dito hiper- $(s ; r)$-nuclear se existem escalares $\left(\lambda_{j}\right)_{j=1}^{\infty} \in \ell_{s}$, $\left(P_{j}\right)_{j=1}^{\infty} \in \ell_{r}^{w}\left(\mathcal{P}\left({ }^{n} E\right)\right)$ e $\left(y_{j}\right)_{j=1}^{\infty} \in \ell_{\infty}(F)$ tais que

$$
P(x)=\sum_{j=1}^{\infty} \lambda_{j} P_{j}(x) y_{j}
$$

para todo $x \in E$. Neste caso escrevemos $P \in \mathcal{P}_{\mathcal{H N}_{(s ; r)}}\left({ }^{n} E ; F\right)$ e definimos a norma hiper$(s ; r)$-nuclear de $P$ por

$$
\|P\|_{\mathcal{P}_{\mathcal{H N}(s ; r)}}=\inf \left\{\left\|\left(\lambda_{j}\right)_{j=1}^{\infty}\right\|_{s} \cdot\left\|\left(P_{j}\right)_{j=1}^{\infty}\right\|_{w, r} \cdot\left\|\left(y_{j}\right)_{j=1}^{\infty}\right\|_{\infty}\right\}
$$

onde o ínfimo é tomado sobre todas as representações como em (4.1).

No caso $s=1$ e $r=\infty$, chamamos os polinômios $n$-homogêneos hiper- $(1 ; \infty)$-nucleares de polinômios hiper-nucleares e denotamos essa classe por $\mathcal{P}_{\mathcal{H N}}\left({ }^{n} E ; F\right)$.

Teorema 4.1.6. Sejam $s \in[1, \infty)$ e $r \in[1, \infty]$ tais que $1 \leq \frac{1}{s}+\frac{1}{r}$. Então a classe $\mathcal{P}_{\mathcal{H N}}(s ; r)$ dos polinômios homogêneos hiper-(s;r)-nucleares é um hiper-ideal de Banach.

Demonstração. Veja [21, Theorem 3.9] ou [72, Teorema 3.2.10].

Precisaremos da seguinte caracterização da norma hiper-nuclear.

Lema 4.1.7. Para todo polinômio n-homogêneo $P \in \mathcal{P}_{\mathcal{H N}}\left({ }^{n} E ; F\right)$, vale que

$$
\|P\|_{\mathcal{P}_{\mathcal{H N}}}=\inf \left\{\sum_{j=1}^{\infty}\left|\lambda_{j}\right| \cdot\left\|P_{j}\right\| \cdot\left\|y_{j}\right\|\right\}
$$

onde o ínfimo é tomado sobre todas as representações hiper-nucleares de P.

Demonstração. A prova é uma adaptação da demonstração de [72, Lema 2.2.11]. 
Seja $P=\sum_{j=1}^{\infty} \lambda_{j} P_{j} \otimes y_{j}$ uma representação hiper-nuclear fixada de $P$. Então,

$$
\begin{aligned}
\sum_{j=1}^{\infty}\left|\lambda_{j}\right| \cdot\left\|P_{j}\right\| \cdot\left\|y_{j}\right\| & \leq\left\|\left(P_{j}\right)_{j=1}^{\infty}\right\|_{\infty} \cdot\left\|\left(y_{j}\right)_{j=1}^{\infty}\right\|_{\infty} \cdot\left(\sum_{j=1}^{\infty}\left|\lambda_{j}\right|\right) \\
& =\left\|\left(P_{j}\right)_{j=1}^{\infty}\right\|_{\infty} \cdot\left\|\left(y_{j}\right)_{j=1}^{\infty}\right\|_{\infty} \cdot\left\|\left(\lambda_{j}\right)_{j=1}^{\infty}\right\|_{1} .
\end{aligned}
$$

Tomando o ínfimo sobre todas as representações de hiper-nucleares de $P$, obtemos

$$
\inf \left\{\sum_{j=1}^{\infty}\left|\lambda_{j}\right| \cdot\left\|P_{j}\right\| \cdot\left\|y_{j}\right\|\right\} \leq\|P\|_{\mathcal{P}_{\mathcal{H N}}} .
$$

Agora suponhamos, sem perda de generalidade, que $P_{j} \neq 0$ e $y_{j} \neq 0$ para todo $j \in \mathbb{N}$. Consideremos as sequências $\left(\mu_{j}\right)_{j=1}^{\infty} \subseteq \mathbb{K},\left(Q_{j}\right)_{j=1}^{\infty} \subseteq \mathcal{P}\left({ }^{n} E\right)$ e $\left(z_{j}\right)_{j=1}^{\infty} \subseteq F$ definidas por

$$
\mu_{j}=\lambda_{j}\left\|P_{j}\right\| \cdot\left\|y_{j}\right\|, \quad Q_{j}=\frac{P_{j}}{\left\|P_{j}\right\|} \text { e } z_{j}=\frac{y_{j}}{\left\|y_{j}\right\|} .
$$

Vejamos que $\sum_{j=1}^{\infty} \mu_{j} Q_{j} \otimes z_{j}$ é uma representação hiper-nuclear de $P$. Primeiro, $\left\|\left(\mu_{j}\right)_{j=1}^{\infty}\right\|_{1}=\sum_{j=1}^{\infty}\left|\mu_{j}\right|=\sum_{j=1}^{\infty}\left|\lambda_{j}\right| \cdot\left\|P_{j}\right\| \cdot\left\|y_{j}\right\| \leq\left\|\left(y_{j}\right)_{j=1}^{\infty}\right\|_{1} \cdot\left\|\left(P_{j}\right)_{j=1}^{\infty}\right\|_{\infty} \cdot\left\|\left(y_{j}\right)_{j=1}^{\infty}\right\|_{\infty}<\infty$.

É imediato que $\left\|\left(Q_{j}\right)_{j=1}^{\infty}\right\|_{\infty}=\left\|\left(z_{j}\right)_{j=1}^{\infty}\right\|_{\infty}=1$ e $\sum_{j=1}^{\infty} \mu_{j} Q_{j} \otimes z_{j}=\sum_{j=1}^{\infty} \lambda_{j} Q_{j} \otimes y_{j}=P$.

Então,

$$
\left\|\left(\mu_{j}\right)_{j=1}^{\infty}\right\|_{1} \cdot\left\|\left(Q_{j}\right)_{j=1}^{\infty}\right\|_{\infty} \cdot\left\|\left(z_{j}\right)_{j=1}^{\infty}\right\|_{\infty}=\sum_{j=1}^{\infty}\left|\lambda_{j}\right| \cdot\left\|P_{j}\right\| \cdot\left\|y_{j}\right\|
$$

e portanto

$$
\left\{\sum_{j=1}^{\infty}\left|\lambda_{j}\right| \cdot\left\|P_{j}\right\| \cdot\left\|y_{j}\right\|\right\} \subseteq\left\{\left\|\left(\mu_{j}\right)_{j=1}^{\infty}\right\|_{1} \cdot\left\|\left(Q_{j}\right)_{j=1}^{\infty}\right\|_{\infty} \cdot\left\|\left(z_{j}\right)_{j=1}^{\infty}\right\|_{\infty}\right\}
$$

Finalmente,

$$
\|P\|_{\mathcal{P}_{\mathcal{H N}}}=\inf \left\{\left\|\left(\mu_{j}\right)_{j=1}^{\infty}\right\|_{1} \cdot\left\|\left(Q_{j}\right)_{j=1}^{\infty}\right\|_{\infty} \cdot\left\|\left(z_{j}\right)_{j=1}^{\infty}\right\|_{\infty}\right\} \leq \inf \left\{\sum_{j=1}^{\infty}\left|\lambda_{j}\right| \cdot\left|P_{j}\right| \cdot\left\|y_{j}\right\|\right\},
$$

donde segue a igualdade desejada. 
Proposição 4.1.8. Sejam $P \in \mathcal{P}_{\mathcal{H N}_{(s ; r)}}\left({ }^{n} E ; F\right) e$

$$
P=\sum_{j=1}^{\infty} \lambda_{j} P_{j} \otimes y_{j}
$$

uma representação hiper-(s;r)-nuclear de P. Então a convergência em (4.2) ocorre na norma $\|\cdot\|_{\mathcal{P}_{\mathcal{H N}(s ; r)}}$.

Demonstração. Sendo (4.2) uma representação hiper- $(s ; r)$-nuclear de $P$, temos $\left(\lambda_{j}\right)_{j=1}^{\infty} \in$ $\ell_{s},\left(P_{j}\right)_{j=1}^{\infty} \in \ell_{r}^{w}\left(\mathcal{P}\left({ }^{n} E\right)\right)$ e $\left(y_{j}\right)_{j=1}^{\infty} \in \ell_{\infty}(F)$.

Como $\mathcal{P}_{\mathcal{H N}_{(s ; r)}}$ é um hiper-ideal de Banach (Teorema 4.1.6), sabemos que $\mathcal{P}_{\mathcal{H N}_{(s ; r)}}\left({ }^{n} E ; F\right)$ é subespaço vetorial de $\mathcal{P}\left({ }^{n} E ; F\right)$, e portanto para $m \in \mathbb{N}$,

$$
\left(P-\sum_{j=1}^{m} \lambda_{j} P_{j} \otimes y_{j}\right) \in \mathcal{P}_{\mathcal{H N}_{(s ; r)}} .
$$

Uma conta rotineira mostra que $\sum_{j=m+1}^{\infty} \lambda_{j} P_{j} \otimes y_{j}$ é uma representação hiper- $(s ; r)$-nuclear de $P-\sum_{j=1}^{m} \lambda_{j} P_{j} \otimes y_{j}, \log \mathrm{O}$

$$
\begin{aligned}
\left\|P-\sum_{j=1}^{m} \lambda_{j} P_{j} \otimes y_{j}\right\|_{\mathcal{P}_{\mathcal{H N}(s ; r)}} & =\left\|\sum_{j=m+1}^{\infty} \lambda_{j} P_{j} \otimes y_{j}\right\|_{\mathcal{P}_{\mathcal{H N}(s ; r)}} \\
& \leq\left\|\left(\lambda_{j}\right)_{j=m+1}^{\infty}\right\|_{s} \cdot\left\|\left(P_{j}\right)_{j=m+1}^{\infty}\right\|_{w, r} \cdot\left\|\left(y_{j}\right)_{j=m+1}^{\infty}\right\|_{\infty} \\
& \leq\left(\sum_{j=m+1}^{\infty}\left|\lambda_{j}\right|^{s}\right)^{\frac{1}{s}} \cdot\left\|\left(P_{j}\right)_{j=1}^{\infty}\right\|_{w, r} \cdot\left\|\left(y_{j}\right)_{j=1}^{\infty}\right\|_{\infty} .
\end{aligned}
$$

Como $\sum_{j=1}^{\infty}\left|\lambda_{j}\right|^{s}<\infty$, fazendo $m \rightarrow \infty$ obtemos

$$
\left\|P-\sum_{j=1}^{m} \lambda_{j} P_{j} \otimes y_{j}\right\|_{\mathcal{P}_{\mathcal{H N}(s ; r)} \longrightarrow 0}
$$

provando que $P=\sum_{j=1}^{\infty} \lambda_{j} P_{j} \otimes y_{j}$ na norma $\|\cdot\|_{\mathcal{P}_{\mathcal{H N}(s ; r)}}$.

Provaremos agora que a hiper-transformada de Borel polinomial só pode ser um isomorfismo sobre um subespaço de $\mathcal{L}\left(\mathcal{P}\left({ }^{n} E\right) ; F^{*}\right)$ no caso dos polinômios hiper-nucleares. 
Teorema 4.1.9. Seja $\mathcal{Q}\left({ }^{n} E ; F\right)$ um subespaço vetorial de $\mathcal{P}\left({ }^{n} E ; F\right)$, munido de uma norma completa $\|\cdot\|_{\mathcal{Q}}$ contendo os polinômios n-homogêneos de posto finito $\mathcal{P}_{\mathcal{F}}\left({ }^{n} E ; F\right)$ e tal que $\|\cdot\| \leq\|\cdot\|_{\mathcal{Q}}$ e $\|P \otimes y\|_{\mathcal{Q}} \leq\|P\| \cdot\|y\|$ para todos $P \in \mathcal{P}\left({ }^{n} E ; F\right)$ e y $\in$ F. Suponha que a hiper-transformada de Borel polinomial

$$
\mathfrak{B}_{n}:\left[\mathcal{Q}\left({ }^{n} E ; F\right)\right]^{*} \longrightarrow \mathcal{L}\left(\mathcal{P}\left({ }^{n} E\right) ; F^{*}\right), \mathfrak{B}_{n}(\phi)(P)(y)=\phi(P \otimes y),
$$

seja um isomorfismo sobre sua imagem. Então $\mathcal{Q}\left({ }^{n} E ; F\right)=\mathcal{P}_{\mathcal{H N}}\left({ }^{n} E ; F\right)$ e $\mathfrak{B}_{n}$ é sobrejetora.

Demonstração. Sejam $P \in \mathcal{P}_{\mathcal{H N}}\left({ }^{n} E ; F\right)$ e $\varepsilon>0$. Pelo Lema 4.1 .7 podemos considerar uma representação hiper-nuclear

$$
P=\sum_{j=1}^{\infty} \lambda_{j} P_{j} \otimes y_{j}
$$

$\operatorname{com}\left(\lambda_{j}\right)_{j=1}^{\infty} \in \ell_{1},\left(P_{j}\right)_{j=1}^{\infty} \in \ell_{\infty}\left(\mathcal{P}\left({ }^{n} E\right)\right)$ e $\left(y_{j}\right)_{j=1}^{\infty} \in \ell_{\infty}(F)$, tal que

$$
\sum_{j=1}^{\infty}\left|\lambda_{j}\right| \cdot\left\|P_{j}\right\| \cdot\left\|y_{j}\right\| \leq(1+\varepsilon) \cdot\|P\|_{\mathcal{P}_{\mathcal{H N}}} .
$$

Chamemos $p_{m}:=\sum_{j=1}^{m} \lambda_{j} P_{j} \otimes y_{j}, m \in \mathbb{N}$. Então, $p_{m} \in \mathcal{P}_{\mathcal{F}}\left({ }^{n} E ; F\right)$ para todo $m \in \mathbb{N}$ e, portanto, $p_{m} \in \mathcal{Q}\left({ }^{n} E ; F\right)$. Como $\sum_{j=1}^{\infty}\left|\lambda_{j}\right| \cdot\left\|P_{j}\right\| \cdot\left\|y_{j}\right\|<\infty$, existe $m_{0} \in \mathbb{N}$ tal que $\sum_{j=k+1}^{\infty}\left|\lambda_{j}\right| \cdot\left\|P_{j}\right\| \cdot\left\|y_{j}\right\|<\varepsilon$ para todo $k \geq m_{0}$. Então, para $m>k \geq m_{0}$,

$$
\begin{aligned}
\left\|p_{m}-p_{k}\right\|_{\mathcal{Q}} & =\left\|\sum_{j=1}^{m} \lambda_{j} P_{j} \otimes y_{j}-\sum_{j=1}^{k} \lambda_{j} P_{j} \otimes y_{j}\right\|_{\mathcal{Q}} \\
& =\left\|\sum_{j=k+1}^{m} \lambda_{j} P_{j} \otimes y_{j}\right\|_{\mathcal{Q}} \\
& \leq \sum_{j=k+1}^{m}\left\|\lambda_{j} P_{j} \otimes y_{j}\right\|_{\mathcal{Q}} \\
& \leq \sum_{j=k+1}^{m}\left|\lambda_{j}\right| \cdot\left\|P_{j}\right\| \cdot\left\|y_{j}\right\|<\varepsilon .
\end{aligned}
$$

Isso prova que a sequência $\left(p_{m}\right)_{m=1}^{\infty}$ é de Cauchy em $\mathcal{Q}\left({ }^{n} E ; F\right)$. Como esse espaço é completo, existe $q \in \mathcal{Q}\left({ }^{n} E ; F\right)$ tal que $p_{m} \longrightarrow q$ na norma $\|\cdot\|_{\mathcal{Q}}$, isto é, $\left\|p_{m}-q\right\|_{\mathcal{Q}} \longrightarrow 0$. Como $\|\cdot\| \leq\|\cdot\|_{\mathcal{Q}}$, concluímos que $\left\|p_{m}-q\right\| \longrightarrow 0$. 
Agora, notemos que

$$
\begin{aligned}
\left\|p_{m}-P\right\| & =\left\|\sum_{j=m+1}^{\infty} \lambda_{j} P_{j} \otimes y_{j}\right\| \\
& =\sup _{\|x\| \leq 1}\left\|\sum_{j=m+1}^{\infty} \lambda_{j} P_{j} \otimes y_{j}(x)\right\| \\
& \leq \sup _{\|x\| \leq 1} \sum_{j=m+1}^{\infty}\left|\lambda_{j}\right| \cdot\left\|P_{j}\right\| \cdot\left\|y_{j}\right\| \cdot\|x\| \\
& =\sum_{j=m+1}^{\infty}\left|\lambda_{j}\right| \cdot\left\|P_{j}\right\| \cdot\left\|y_{j}\right\|<\varepsilon,
\end{aligned}
$$

ou seja, $\left\|p_{m}-P\right\| \longrightarrow 0$. Segue que $P=q \in \mathcal{Q}\left({ }^{n} E ; F\right)$. Mais ainda,

$$
P=q=\lim _{m \rightarrow \infty} p_{m}=\sum_{j=1}^{\infty} \lambda_{j} P_{j} \otimes y_{j}
$$

na norma $\|\cdot\|_{\mathcal{Q}}$, e portanto

$\|P\|_{\mathcal{Q}}=\left\|\sum_{j=1}^{\infty} \lambda_{j} P_{j} \otimes y_{j}\right\|_{\mathcal{Q}} \leq \sum_{j=1}^{\infty}\left|\lambda_{j}\right| \cdot\left\|P_{j} \otimes y_{j}\right\|_{\mathcal{Q}} \leq \sum_{j=1}^{\infty}\left|\lambda_{j}\right| \cdot\left\|P_{j}\right\| \cdot\left\|y_{j}\right\| \leq(1+\varepsilon) \cdot\|P\|_{\mathcal{P}_{\mathcal{H N}}}$.

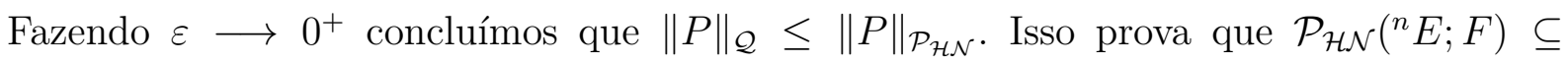
$\mathcal{Q}\left({ }^{n} E ; F\right)$ e que o operador inclusão $i: \mathcal{P}_{\mathcal{H N}}\left({ }^{n} E ; F\right) \hookrightarrow \mathcal{Q}\left({ }^{n} E ; F\right)$ é contínuo.

Para a verificação da inclusão oposta, tome

$$
T: \mathcal{P}\left({ }^{n} E\right) \times F \longrightarrow \mathcal{P}_{\mathcal{H N}}\left({ }^{n} E ; F\right), T(P, y)=P \otimes y
$$

É imediato que $T$ é um operador bilinear e contínuo. Pela propriedade universal do produto tensorial projetivo (Teorema 1.2.13), existe um operador linear e contínuo

$$
T_{L}: \mathcal{P}\left({ }^{n} E\right) \widehat{\otimes}_{\pi} F \longrightarrow \mathcal{P}_{\mathcal{H N}}\left({ }^{n} E ; F\right)
$$

tal que

$$
T_{L}(P \otimes y)=T(P, y)=P \otimes y \text { para todos } P \in \mathcal{P}\left({ }^{n} E\right) \text { e } y \in F
$$

Consideremos as aplicações

$$
\alpha_{1}:\left(\mathcal{P}\left({ }^{n} E\right) \widehat{\otimes}_{\pi} F\right)^{*} \longrightarrow \mathcal{L}\left(\mathcal{P}\left({ }^{n} E\right), F ; \mathbb{K}\right), \alpha_{1}(\varphi)(P, y)=\varphi(P \otimes y)
$$


e

$$
\alpha_{2}: \mathcal{L}\left(\mathcal{P}\left({ }^{n} E\right), F ; \mathbb{K}\right) \longrightarrow \mathcal{L}\left(\mathcal{P}\left({ }^{n} E\right) ; F^{*}\right), \alpha_{2}(\xi)(P)(y)=\xi(P, y)
$$

Pelo Teorema 1.2.13, $\alpha_{1}$ é um isomorfismo isométrico e, pela Proposição 1.2.6(b), $\alpha_{2}$ também é um isomorfismo isométrico. Portanto,

$$
\psi:=\alpha_{2} \circ \alpha_{1}:\left(\mathcal{P}\left({ }^{n} E\right) \widehat{\otimes}_{\pi} F\right)^{*} \longrightarrow \mathcal{L}\left(\mathcal{P}\left({ }^{n} E\right) ; F^{*}\right), \psi(\varphi)(P)(y)=\varphi(P \otimes y),
$$

é um isomorfismo isométrico.

Considerando a cadeia

$$
\mathcal{P}\left({ }^{n} E\right) \widehat{\otimes}_{\pi} F \stackrel{T_{L}}{\longrightarrow} \mathcal{P}_{\mathcal{H N}}\left({ }^{n} E ; F\right) \stackrel{i}{\longrightarrow} \mathcal{Q}\left({ }^{n} E ; F\right)
$$

podemos definir $u:=i \circ T_{L}$, isto é,

$$
u: \mathcal{P}\left({ }^{n} E\right) \widehat{\otimes}_{\pi} F \longrightarrow \mathcal{Q}\left({ }^{n} E ; F\right), u(P \otimes y)=\left(i \circ T_{L}\right)(P \otimes y)=T(P, y) .
$$

Assim, seu adjunto é dado por

$$
u^{*}:\left[\mathcal{Q}\left({ }^{n} E ; F\right)\right]^{*} \longrightarrow\left(\mathcal{P}\left({ }^{n} E\right) \widehat{\otimes}_{\pi} F\right)^{*}, u^{*}(\phi)(P \otimes y)=\phi(u(P \otimes y))=\phi(T(P, y)) .
$$

E considerando a cadeia

$$
\left[\mathcal{Q}\left({ }^{n} E ; F\right)\right]^{*} \stackrel{u^{*}}{\longrightarrow}\left(\mathcal{P}\left({ }^{n} E\right) \widehat{\otimes}_{\pi} F\right)^{*} \stackrel{\psi}{\longrightarrow} \mathcal{L}\left(\mathcal{P}\left({ }^{n} E\right) ; F^{*}\right)
$$

podemos considerar a composição

$$
\psi \circ u^{*}:\left[\mathcal{Q}\left({ }^{n} E ; F\right)\right]^{*} \longrightarrow \mathcal{L}\left(\mathcal{P}\left({ }^{n} E\right) ; F^{*}\right) .
$$

Dado $\phi \in\left[\mathcal{Q}\left({ }^{n} E ; F\right)\right]^{*}$, para todos $P \in \mathcal{P}\left({ }^{n} E\right), y \in F$

$$
\left(\psi \circ u^{*}\right)(\phi)(P)(y)=\psi\left(u^{*}(\phi)\right)(P)(y)=u^{*}(\phi)(P \otimes y)=\phi(P \otimes y)=\mathfrak{B}_{n}(\phi)(P)(y),
$$

provando que $\psi \circ u^{*}=\mathfrak{B}_{n}$. Por hipótese, $\psi \circ u^{*}$ é um isomorfismo isométrico sobre sua imagem. Em particular, é injetor e sendo $\psi$ um isomorfismo, concluímos que $u^{*}$ é injetor.

É claro que

$$
\psi^{-1}: \mathcal{L}\left(\mathcal{P}\left({ }^{n} E\right) ; F^{*}\right) \longrightarrow\left(\mathcal{P}\left({ }^{n} E\right) \widehat{\otimes}_{\pi} F\right)^{*}
$$

é um isomorfismo isométrico, pois $\psi$ o é. Logo, sua restrição

$$
\theta:=\left.\psi^{-1}\right|_{\mathfrak{B}_{n}\left(\mathcal{Q}\left({ }^{n} E ; F\right)^{*}\right)}: \mathfrak{B}_{n}\left(\mathcal{Q}\left({ }^{n} E ; F\right)^{*}\right) \longrightarrow \psi^{-1}\left(\mathfrak{B}_{n}\left(\mathcal{Q}\left({ }^{n} E ; F\right)^{*}\right)\right)
$$


é também um isomorfismo isométrico.

Como $\left[\mathcal{Q}\left({ }^{n} E ; F\right)\right]^{*}$ é espaço de Banach e $\mathfrak{B}_{n}$ é isomorfismo sobre sua imagem, temos que $\mathfrak{B}_{n}\left(\mathcal{Q}\left({ }^{n} E ; F\right)^{*}\right)$ é espaço de Banach. Logo,

$$
\theta\left(\mathfrak{B}_{n}\left(\mathcal{Q}\left({ }^{n} E ; F\right)^{*}\right)\right)=\psi^{-1}\left(\mathfrak{B}_{n}\left(\mathcal{Q}\left({ }^{n} E ; F\right)^{*}\right)\right)
$$

é espaço Banach e, portanto, subespaço fechado de $\left(\mathcal{P}\left({ }^{n} E\right) \widehat{\otimes}_{\pi} F\right)^{*}$.

De $\psi \circ u^{*}=\mathfrak{B}_{n}$ decorre que $u^{*}=\psi^{-1} \circ \mathfrak{B}_{n}$. Para todo $\phi \in\left[\mathcal{Q}\left({ }^{n} E ; F\right)\right]^{*}$,

$$
u^{*}(\phi)=\left(\psi^{-1} \circ \mathfrak{B}_{n}\right)(\phi)=\psi^{-1}\left(\mathfrak{B}_{n}(\phi)\right)=\theta\left(\mathfrak{B}_{n}(\phi)\right)
$$

e portanto

$$
u^{*}\left(\mathcal{Q}\left({ }^{n} E ; F\right)^{*}\right)=\theta\left(\mathfrak{B}_{n}\left(\mathcal{Q}\left({ }^{n} E ; F\right)^{*}\right)\right)
$$

é subespaço fechado de $\left(\mathcal{P}\left({ }^{n} E\right) \widehat{\otimes}_{\pi} F\right)^{*}$. Assim, $u^{*}$ é injetor e tem imagem fechada. Pelo Teorema da Aplicação Aberta segue que $u^{*}$ é um isomorfismo sobre sua imagem. Pelo Teorema 1.1.7(a) concluímos que $u=i \circ T_{L}$ é sobrejetor. Portanto, $i$ é sobrejetor, o que prova que $\mathcal{P}_{\mathcal{H N}}\left({ }^{n} E ; F\right)=\mathcal{Q}\left({ }^{n} E ; F\right)$.

Provemos agora que $\mathfrak{B}_{n}$ é sobrejetora. Dado $v \in \mathcal{L}\left(\mathcal{P}\left({ }^{n} E\right) ; F^{*}\right)$, consideremos a linearização $A_{L}: \mathcal{P}\left({ }^{n} E\right) \widehat{\otimes}_{\pi} F \longrightarrow \mathbb{K}$ do operador bilinear contínuo

$$
A: \mathcal{P}\left({ }^{n} E\right) \times F \longrightarrow \mathbb{K}, A(Q, y)=v(Q)(y)
$$

Usando o fato de que a correspondência $\mathcal{P}_{\mathcal{F}}\left({ }^{n} E ; F\right) \longleftrightarrow \mathcal{P}\left({ }^{n} E\right) \otimes F$ é um isomorfismo entre espaços vetoriais, podemos considerar a restrição $\varphi$ de $A_{L}$ a

$$
\mathcal{P}\left({ }^{n} E\right) \otimes F \subseteq \mathcal{P}_{\mathcal{H N}}\left({ }^{n} E ; F\right)
$$

isto é,

$$
\varphi:\left(\mathcal{P}\left({ }^{n} E\right) \otimes F,\|\cdot\|_{\mathcal{H N}}\right) \longrightarrow \mathbb{K}, \varphi(B)=A_{L}(B) .
$$

É claro que $\varphi$ é linear. Provemos que é contínuo. Seja $P \in \mathcal{P}\left({ }^{n} E\right) \otimes F$. Dado $\varepsilon>0$, pelo Lema 4.1.7 podemos tomar $P=\sum_{j=1}^{\infty} \lambda_{j} P_{j} \otimes y_{j}$ uma representação hiper-nuclear de $P$ tal que $\sum_{j=1}^{\infty}\left|\lambda_{j}\right| \cdot\left\|P_{j}\right\| \cdot\left\|y_{j}\right\| \leq(1+\varepsilon)\|P\|_{\mathcal{P}_{\mathcal{H N}}} \cdot$ Como

$$
\sum_{j=1}^{\infty} \pi\left(\lambda_{j} P_{j} \otimes y_{j}\right)=\sum_{j=1}^{\infty}\left|\lambda_{j}\right| \cdot\left\|P_{j} \otimes y_{j}\right\|=\sum_{j=1}^{\infty}\left|\lambda_{j}\right| \cdot\left\|P_{j}\right\| \cdot\left\|y_{j}\right\|<+\infty
$$


a série $\sum_{j=1}^{\infty} \lambda_{j} P_{j} \otimes y_{j}$ é absolutamente convergente em $\mathcal{P}\left({ }^{n} E\right) \widehat{\otimes}_{\pi} F$, logo convergente. Assim, a convergência $P=\sum_{j=1}^{\infty} \lambda_{j} P_{j} \otimes y_{j}$ se dá na norma $\pi$ de $\mathcal{P}\left({ }^{n} E\right) \widehat{\otimes}_{\pi} F$. Conforme mostrado no início desta demonstração, essa convergência também se dá na norma $\|\cdot\|_{\mathcal{P}_{\mathcal{H N}}}$. Da continuidade de $A_{L}$ temos

$$
\begin{aligned}
|\varphi(P)|=\left|A_{L}(P)\right| & =\left|A_{L}\left(\sum_{j=1}^{\infty} \lambda_{j} P_{j} \otimes y_{j}\right)\right| \\
& \leq\left\|A_{L}\right\| \cdot \pi\left(\sum_{j=1}^{\infty} \lambda_{j} P_{j} \otimes y_{j}\right) \\
& \leq\left\|A_{L}\right\| \cdot \sum_{j=1}^{\infty} \pi\left(\lambda_{j} P_{j} \otimes y_{j}\right) \\
& =\left\|A_{L}\right\| \cdot \sum_{j=1}^{\infty}\left|\lambda_{j}\right| \cdot\left\|P_{j}\right\| \cdot\left\|y_{j}\right\| \\
& \leq(1+\varepsilon)\left\|A_{L}\right\| \cdot\|P\|_{\mathcal{P}_{\mathcal{H N}} .}
\end{aligned}
$$

Isso mostra que o funcional $\varphi$ é contínuo com respeito à norma $\|\cdot\|_{\mathcal{P}_{\mathcal{H N}}}$, isto é, $\varphi \in$ $\left(\mathcal{P}\left({ }^{n} E\right) \otimes F,\|\cdot\|_{\mathcal{P}_{\mathcal{H N}}}\right)^{*}$. Usando mais uma vez que mostramos no início da demonstração que somas parciais de representações hiper-nucleares convergem na norma $\|\cdot\|_{\mathcal{H N}}$ para o polinômio hiper-nuclear, segue que $\mathcal{P}\left({ }^{n} E\right) \otimes F$ é denso em $\mathcal{P}_{\mathcal{H N}}\left({ }^{n} E ; F\right)$. Seja então $\tilde{\varphi}$ a (única) extensão linear e contínua de $\varphi$ a $\mathcal{P}_{\mathcal{H N}}\left({ }^{n} E ; F\right)$. Para todos $Q \in \mathcal{P}\left({ }^{n} E\right)$ e $y \in F$,

$$
\mathfrak{B}_{n}(\tilde{\varphi})(Q)(y)=\tilde{\varphi}(Q \otimes y)=\varphi(Q \otimes y)=A_{L}(Q \otimes y)=A(Q, y)=v(Q)(y),
$$

isto é, $\mathfrak{B}_{n}(\tilde{\varphi})=v$, o que prova a sobrejetividade de $\mathfrak{B}_{n}$ e completa a demonstração.

Exemplo 4.1.10. Conforme vimos no Exemplo 4.1.3, para todo hiper-ideal de Banach de polinômios $\left(\mathcal{Q},\|\cdot\|_{\mathcal{Q}}\right)$, a componente $\left(\mathcal{Q}\left({ }^{n} E ; F\right),\|\cdot\|_{\mathcal{Q}}\right)$ satisfaz as condições do Teorema 4.1.9 para todos $n, E$ e $F$.

\subsection{Ideais de polinômios de composição}

O objetivo desta seção é a obtenção de uma versão polinomial do Teorema 3.2.1. No próximo resultado veremos que a parte mais usada do teorema, que permite obter representações não lineares a partir de representações lineares, funciona bem; fato este que 
nos permitirá seguir com as aplicações. A análise da outra implicação do caso polinomial do Teorema 3.2.1 revelará uma diferença inesperada entre os casos multilinear e polinomial (veja Observações 4.2.1 e 4.2.5).

Dado um ideal de Banach de operadores $\left(\mathcal{I},\|\cdot\|_{\mathcal{I}}\right)$, a partir de agora trabalharemos com o ideal de polinômios $\mathcal{I} \circ \mathcal{P}$ munido com a norma que foi denotada por $\|\cdot\|_{\mathcal{I}_{\circ}, 2}$ na Definição 1.6.15 mas que, por simplicidade, passaremos a denotar por $\|\cdot\|_{\mathcal{I} \circ \mathcal{P}}$ a partir de agora. Assim, para $P \in \mathcal{I} \circ \mathcal{P}\left({ }^{n} E ; F\right)$, chamando de $P_{L, s}$ sua linearização no produto tensorial projetivo com a norma $s$-projetiva, isto é, $P_{L, s} \in \mathcal{I}\left(\widehat{\otimes}_{\pi_{s}}^{n, s} E ; F\right)$, de acordo com a Definição 1.6.15 e com a Proposição 1.6.16,

$$
\|P\|_{\mathcal{I} \circ \mathcal{P}}=\left\|P_{L, s}\right\|_{\mathcal{I}}=\inf \left\{\|u\|_{\mathcal{I}} \cdot\|Q\|: P=u \circ Q, Q \in \mathcal{P}\left({ }^{n} E ; G\right) \text { e } u \in \mathcal{I}(G ; F)\right\} .
$$

Por ser um hiper-ideal de polinômios, $\mathcal{I} \circ \mathcal{P}$ é um candidato natural para a aplicação da hiper-transformada de Borel polinomial.

Observação 4.2.1. Em vista do Teorema 3.2.1, neste ponto o esperado é que as seguintes condições sejam equivalentes:

(i) A transformada de Borel linear $\beta=\mathfrak{B}$ representa funcionais lineares em $\mathcal{I}(E ; F)$.

(ii) A hiper-transformada de Borel polinomial $\mathfrak{B}_{n}$ representa funcionais lineares em $\mathcal{I}$ 。 $\mathcal{P}\left({ }^{n} E ; F\right)$ para todo $n$.

(iii) A hiper-transformada de Borel polinomial $\mathfrak{B}_{n}$ representa funcionais lineares em $\mathcal{I} \circ$ $\mathcal{P}\left({ }^{n} E ; F\right)$ para algum $n$.

Por um lado, (i) implica (ii) (veja Teorema 4.2.3), e esse fato nos permitirá seguir adiante com as aplicações. Por outro lado, não sabemos se (iii) implica (i). Na verdade acreditamos que não implica, e a razão é que a demonstração da implicação multilinear correspondente não funciona para polinômios. Mais especificamente, o argumento central utilizado na demonstração da implicação $(c) \Longrightarrow(a)$ do Teorema 3.2.1 envolveu o operador

$$
v: E^{*} \longrightarrow \mathcal{L}(E, \mathbb{K}, \stackrel{(n-1)}{\cdots}, \mathbb{K}), v\left(x^{*}\right)\left(x, \lambda_{1}, \ldots, \lambda_{n-1}\right)=\lambda_{1} \cdots \lambda_{n-1} x^{*}(x),
$$

o qual obviamente não tem como ser adaptado para o contexto polinomial. Um resultado parcial nessa direção será apresentado no Teorema 4.2.4. Acreditamos que esse fenômeno é apenas mais uma diferença entre as teorias multilinear e polinomial. E são essas diferenças que fazem com que ambas as teorias mereçam ser estudadas. Voltaremos a essa questão na Observação 4.2.5.

Antes de provarmos a implicação que funciona bem no caso polinomial, precisamos do lema a seguir que, embora conhecido e de fácil demonstração, nos será muito útil. 
Lema 4.2.2. Para quaisquer espaços de Banach $E$ e $F$, se $P \in \mathcal{P}\left({ }^{n} E\right)$ e $y \in F$, então $(P \otimes y)_{L, s}=P_{L, s} \otimes y$.

Teorema 4.2.3. Sejam $\left(\mathcal{I},\|\cdot\|_{\mathcal{I}}\right)$ um ideal de Banach, $\alpha$ um semi-ideal à esquerda e $P_{1}$ e $P_{2}$ propriedades geométricas de espaços de Banach. Suponha que para todos espaços de Banach $E$ e $F$ tais que $E^{*}$ tem $P_{1}$ e $F$ tem $P_{2}$, a transformada de Borel linear

$$
\mathfrak{B}:\left(\mathcal{I}(E, F),\|\cdot\|_{\mathcal{I}}\right)^{*} \longrightarrow \mathcal{L}_{\alpha}\left(E^{*} ; F^{*}\right), \mathfrak{B}(\phi)\left(x^{*}\right)(y)=\phi\left(x^{*} \otimes y\right),
$$

seja um isomorfismo isométrico. Então, para todo $n \in \mathbb{N}$ e todos espaços de Banach $E$ e $F$ tais que $\mathcal{P}\left({ }^{n} E\right)$ tem $P_{1}$ e $F$ tem $P_{2}$, a hiper-transformada de Borel polinomial

$$
\mathfrak{B}_{n}:\left(\mathcal{I} \circ \mathcal{P}\left({ }^{n} E ; F\right),\|\cdot\|_{\mathcal{I} \circ \mathcal{P}}\right)^{*} \longrightarrow \mathcal{L}_{\alpha}\left(\mathcal{P}\left({ }^{n} E\right) ; F^{*}\right), \mathfrak{B}_{n}(\phi)(P)(y)=\phi(P \otimes y),
$$

é um isomorfismo isométrico.

Demonstração. Nesta demonstração, as componentes de $\mathcal{I}$ são munidas com a norma $\|\cdot\|_{\mathcal{I}}$ e as componentes de $\mathcal{I} \circ \mathcal{P}$ com a norma $\|\cdot\|_{\mathcal{I} \circ \mathcal{P}}$.

Seja $n \in \mathbb{N}$ e suponhamos que $\mathcal{P}\left({ }^{n} E\right)$ tenha $P_{1}$ e que $F$ tenha $P_{2}$. Pelas Proposições 1.6.16 e 1.6.14, sabemos que o operador linearização

$$
L: \mathcal{I} \circ \mathcal{P}\left({ }^{n} E ; F\right) \longrightarrow \mathcal{I}\left(\hat{\otimes}_{\pi_{s}}^{n, s} E ; F\right), L(P)=P_{L, s},
$$

é um isomorfismo isométrico. Logo, o adjunto de seu inverso

$$
\left(L^{-1}\right)^{*}:\left[\mathcal{I} \circ \mathcal{P}\left({ }^{n} E ; F\right)\right]^{*} \longrightarrow\left[\mathcal{I}\left(\hat{\otimes}_{\pi_{s}}^{n, s} E ; F\right)\right]^{*}
$$

também é um isomorfismo isométrico. Pelo Teorema 1.6.7 sabemos que o operador linearização $\ell: \mathcal{P}\left({ }^{n} E\right) \longrightarrow\left(\widehat{\otimes}_{\pi_{s}}^{n, s} E\right)^{*}$ é um isomorfismo isométrico. Portanto $\left(\widehat{\otimes}_{\pi_{s}}^{n, s} E\right)^{*}$ possui a propriedade $P_{1}$ e, por hipótese, a transformada de Borel linear

$$
\mathfrak{B}:\left[\mathcal{I}\left(\widehat{\otimes}_{\pi_{s}}^{n, s} E ; F\right)\right]^{*} \longrightarrow \mathcal{L}_{\alpha}\left(\left(\widehat{\otimes}_{\pi_{s}}^{n, s} E\right)^{*} ; F^{*}\right)
$$

é um isomorfismo isométrico.

Pelo Lema 3.1.8 sabemos que o operador de composição

$$
J_{\ell}: \mathcal{L}_{\alpha}\left(\left(\widehat{\otimes}_{\pi_{s}}^{n, s} E\right)^{*} ; F^{*}\right) \longrightarrow \mathcal{L}_{\alpha}\left(\mathcal{P}\left({ }^{n} E\right) ; F^{*}\right), J_{\ell}(u)=u \circ \ell,
$$

é também um isomorfismo isométrico. É suficiente então provar que o diagrama

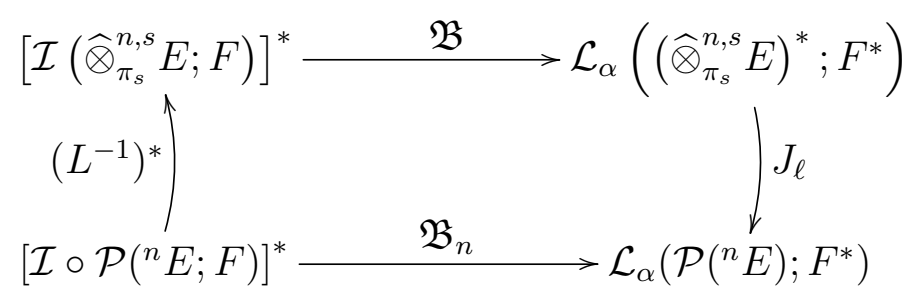


é comutativo. Para isso sejam $\phi \in\left[\mathcal{I} \circ \mathcal{P}\left({ }^{n} E ; F\right)\right]^{*}, P \in \mathcal{P}\left({ }^{n} E\right)$ e $y \in F$. Usando que (Lema 4.2.2 e Teorema 1.6.7)

$$
L(P \otimes y)=(P \otimes y)_{L, s}=P_{L, s} \otimes y
$$

obtemos

$$
\begin{aligned}
\left(J_{\ell} \circ \mathfrak{B} \circ\left(L^{-1}\right)^{*}\right)(\phi)(P)(y) & =\left(\left(J_{\ell}\left(\mathfrak{B}\left(\left(L^{-1}\right)^{*}(\phi)\right)\right)\right)(P)\right)(y) \\
& =\left(\left(\mathfrak{B}\left(\left(L^{-1}\right)^{*}(\phi)\right)\right)\left(P_{L, s}\right)\right)(y) \\
& =\left(L^{-1}\right)^{*}(\phi)\left(P_{L, s} \otimes y\right) \\
& =\phi\left(\left(L^{-1}\right)\left(P_{L, s} \otimes y\right)\right) \\
& =\phi(P \otimes y)=\mathfrak{B}_{n}(\phi)(P)(y),
\end{aligned}
$$

o que prova a comutatividade do diagrama e completa a demonstração.

Vimos na Observação 4.2.1 que não é possível adaptar a demonstração do caso multilinear da recíproca do teorema acima para o caso polinomial. Tentamos então uma abordagem diferente para o caso polinomial, e o que conseguimos se encontra no teorema a seguir, o qual pode ser interpretado como uma recíproca parcial do Teorema 4.2.3.

Teorema 4.2.4. Sejam $\left(\mathcal{I},\|\cdot\|_{\mathcal{I}}\right)$ um ideal de Banach, $\alpha$ um semi-ideal à esquerda e $E$ e $F$ espaços de Banach. Suponha que exista $n \in \mathbb{N}$ tal que a hiper-transformada de Borel polinomial

$$
\mathfrak{B}_{n}:\left(\mathcal{I} \circ \mathcal{P}\left({ }^{n} E ; F\right),\|\cdot\|_{\mathcal{I} \circ \mathcal{P}}\right)^{*} \longrightarrow \mathcal{L}_{\alpha}\left(\mathcal{P}\left({ }^{n} E\right) ; F^{*}\right), \mathfrak{B}_{n}(\phi)(P)(y)=\phi(P \otimes y)
$$

seja um isomorfismo sobre sua imagem. Então, a transformada de Borel linear

$$
\mathfrak{B}:\left(\mathcal{I}(E ; F),\|\cdot\|_{\mathcal{I}}\right)^{*} \longrightarrow \mathcal{L}_{\alpha}\left(E^{*} ; F^{*}\right), \mathfrak{B}(\phi)\left(x^{*}\right)(y)=\phi\left(x^{*} \otimes y\right)
$$

é também um isomorfismo sobre sua imagem.

Demonstração. Nesta demonstração as componentes de $\mathcal{I}$ são munidas com a norma $\|\cdot\|_{\mathcal{I}}$ e as componentes de $\mathcal{I} \circ \mathcal{P}$ com a norma $\|\cdot\|_{\mathcal{I} \circ \mathcal{P}}$.

Pela Proposição 1.6.11 existe um operador $u: E^{*} \longrightarrow \mathcal{P}\left({ }^{n} E\right)$ que é um isomorfismo sobre um subespaço complementado de $\mathcal{P}\left({ }^{n} E\right)$. Em particular, $u\left(E^{*}\right)$ é um subespaço fechado de $\mathcal{P}\left({ }^{n} E\right)$.

Pelo Teorema 1.6.8 existe um operador $E \longrightarrow \widehat{\otimes}_{\pi}^{n, s} E$ que é um isomorfismo sobre um subespaço complementado de $\widehat{\otimes}_{\pi}^{n, s} E$. Como as normas $\pi$ e $\pi_{s}$ são equivalentes [42, p. 165], 
podemos tomar $i: E \longrightarrow \widehat{\otimes}_{\pi_{s}}^{n, s} E$ um isomorfismo sobre um subespaço complementado de $\widehat{\otimes}_{\pi_{s}}^{n, s} E$.

Vejamos que, considerando o operador linearização

$$
\ell: \mathcal{P}\left({ }^{n} E\right) \longrightarrow\left(\widehat{\otimes}_{\pi_{s}}^{n, s} E\right)^{*}
$$

que é um isomorfismo isométrico (Teorema 1.6.7), podemos tomar os operadores $u$ e $i$ tais que $i^{*} \circ \ell \circ u=i d_{E^{*}}$.

$$
E^{*} \stackrel{u}{\longrightarrow} \mathcal{P}\left({ }^{n} E\right) \stackrel{\ell}{\longrightarrow}\left(\widehat{\otimes}_{\pi_{s}}^{n, s} E\right)^{*} \stackrel{i^{*}}{\longrightarrow} E^{*}
$$

Vamos provar para $n=2$, o caso geral é análogo, apenas com notação mais pesada e manipulação algébrica mais longa. Escolha $e \in E$ e $\psi \in E^{*}$ tais que $\psi(e)=1$. O operador $u$ de Aron-Schottenloher (Proposição 1.6.11) é dado por

$$
u: E^{*} \longrightarrow \mathcal{P}\left({ }^{2} E\right), u\left(x^{*}\right)(x)=\psi(x) x^{*}(x) .
$$

E o operador $i$ de Blasco (Teorema 1.6.8) é dado por

$$
i: E \longrightarrow \widehat{\otimes}_{\pi_{s}}^{2, s} E, i(x)=2 e \otimes_{s} x-\psi(x) e \otimes e .
$$

Relembre que $e \otimes_{s} x=\frac{e \otimes x+x \otimes e}{2}$ e note que

$$
\begin{aligned}
& (e+x) \otimes(e+x)=e \otimes e+e \otimes x+x \otimes e+x \otimes x, \\
& (e-x) \otimes(e-x)=e \otimes e-e \otimes x-x \otimes e+x \otimes x .
\end{aligned}
$$

Subtraindo as duas equações acima, obtemos

$$
(e+x) \otimes(e+x)-(e-x) \otimes(e-x)=2(e \otimes x+x \otimes e)=4 e \otimes_{s} x .
$$

Para $x^{*} \in E^{*}$ e $x \in E$,

$$
\begin{aligned}
\left(i^{*} \circ \ell \circ u\right)\left(x^{*}\right)(x) & =i^{*}\left(\ell\left(u\left(x^{*}\right)\right)\right)(x)=i^{*}\left(u\left(x^{*}\right)_{L}\right)(x) \\
& =u\left(x^{*}\right)_{L}\left(2 e \otimes_{s} x-\psi(x) e \otimes e\right) \\
& =u\left(x^{*}\right)_{L}\left(2 e \otimes_{s} x\right)-\psi(x) u\left(x^{*}\right)_{L}(e \otimes e) \\
& =u\left(x^{*}\right)_{L}\left(2 e \otimes_{s} x\right)-\psi(x) u\left(x^{*}\right)(e) \\
& =u\left(x^{*}\right)_{L}\left(2 e \otimes_{s} x\right)-\psi(x) \psi(e) x^{*}(e) \\
& =u\left(x^{*}\right)_{L}\left(2 e \otimes_{s} x\right)-\psi(x) x^{*}(e) \\
& =u\left(x^{*}\right)_{L}\left(\frac{(e+x) \otimes(e+x)-(e-x) \otimes(e-x)}{2}\right)-\psi(x) x^{*}(e)
\end{aligned}
$$




$$
\begin{aligned}
= & \frac{1}{2} u\left(x^{*}\right)_{L}((e+x) \otimes(e+x)-(e-x) \otimes(e-x))-\psi(x) x^{*}(e) \\
= & \frac{1}{2}\left[u\left(x^{*}\right)_{L}((e+x) \otimes(e+x))-u\left(x^{*}\right)_{L}((e-x) \otimes(e-x))\right]-\psi(x) x^{*}(e) \\
= & \frac{1}{2}\left[u\left(x^{*}\right)(e+x)-u\left(x^{*}\right)(e-x)\right]-\psi(x) x^{*}(e) \\
= & \frac{1}{2}\left[\psi(e+x) x^{*}(e+x)-\psi(e-x) x^{*}(e-x)\right]-\psi(x) x^{*}(e) \\
= & \frac{1}{2}\left[\psi(e) x^{*}(e)+\psi(e) x^{*}(x)+\psi(x) x^{*}(e)+\psi(x) x^{*}(x)-\psi(e) x^{*}(e)+\right. \\
& \left.+\psi(e) x^{*}(x)+\psi(x) x^{*}(e)-\psi(x) x^{*}(x)\right]-\psi(x) x^{*}(e) \\
= & \psi(e) x^{*}(x)+\psi(x) x^{*}(e)-\psi(x) x^{*}(e) \\
= & \psi(e) x^{*}(x)=x^{*}(x) .
\end{aligned}
$$

Provamos que $\left(i^{*} \circ \ell \circ u\right)\left(x^{*}\right)=x^{*}$ para todo $x^{*} \in E^{*}$, isto é, $i^{*} \circ \ell \circ u=i d_{E^{*}}$, conforme o desejado.

Como $\alpha$ é um semi-ideal à esquerda, as aplicações

$$
\begin{gathered}
J_{u}: \mathcal{L}_{\alpha}\left(\mathcal{P}\left({ }^{n} E\right) ; F^{*}\right) \longrightarrow \mathcal{L}_{\alpha}\left(E^{*} ; F^{*}\right), J_{u}(T)=T \circ u, \\
K: \mathcal{L}_{\alpha}\left(E^{*} ; F^{*}\right) \longrightarrow \mathcal{L}_{\alpha}\left(\mathcal{P}\left({ }^{n} E\right) ; F^{*}\right), K(t)=t \circ i^{*} \circ \ell,
\end{gathered}
$$

são operadores lineares contínuos bem definidos. Vejamos que, além disso, $K$ é um isomorfismo sobre o subespaço complementado

$$
Y:=\left\{K(t): t \in \mathcal{L}_{\alpha}\left(E^{*} ; F^{*}\right)\right\}=\left\{t \circ i^{*} \circ \ell: t \in \mathcal{L}_{\alpha}\left(E^{*} ; F^{*}\right)\right\}
$$

de $\mathcal{L}_{\alpha}\left(\mathcal{P}\left({ }^{n} E\right) ; F^{*}\right)$. Com efeito,

$$
\left(J_{u} \circ K\right)(t)=J_{u}(K(t))=J_{u}\left(t \circ i^{*} \circ \ell\right)=t \circ i^{*} \circ \ell \circ u=t \circ i d_{E^{*}}=t,
$$

para todo $t \in \mathcal{L}_{\alpha}\left(E^{*} ; F^{*}\right)$, provando que $J_{u} \circ K$ é o operador identidade em $\mathcal{L}_{\alpha}\left(E^{*} ; F^{*}\right)$. Pelo Lema 1.1.14 concluímos que $Y$ é um subespaço complementado, portanto fechado, de $\mathcal{L}_{\alpha}\left(\mathcal{P}\left({ }^{n} E\right) ; F^{*}\right)$, e que $K: \mathcal{L}_{\alpha}\left(E^{*} ; F^{*}\right) \longrightarrow Y$ é um isomorfismo cuja inversa é a restrição de $J_{u}$ a $Y$. Em particular, $J_{u}: Y \longrightarrow \mathcal{L}_{\alpha}\left(E^{*} ; F^{*}\right)$ é um isomorfismo.

Como $\mathcal{I}$ é um ideal de Banach, a aplicação

$$
J_{i}: \mathcal{I}\left(\widehat{\otimes}_{\pi_{s}}^{n, s} E ; F\right) \longrightarrow \mathcal{I}(E ; F), J_{i}(v)=v \circ i
$$

é um operador linear bem definido. Vejamos que $J_{i}$ é contínuo: para $v \in \mathcal{I}\left(\widehat{\otimes}_{\pi_{s}}^{n, s} E ; F\right)$,

$$
\left\|J_{i}(v)\right\|_{\mathcal{I}}=\|v \circ i\|_{\mathcal{I}} \leq\|v\|_{\mathcal{I}} \cdot\|i\|
$$


Como $i(E)$ é um subespaço complementado de $\widehat{\otimes}_{\pi_{s}}^{n, s} E$, seja $\rho: \widehat{\otimes}_{\pi_{s}}^{n, s} E \longrightarrow E$ a projeção correspondente, isto é, $\rho \circ i=i d_{E}$. Dado $t \in \mathcal{I}(E ; F)$, a composição

$$
\widehat{\otimes}_{\pi_{s}}^{n, s} E \stackrel{\rho}{\longrightarrow} E \stackrel{t}{\longrightarrow} F
$$

pertence ao ideal $\mathcal{I}$, isto é, $t \circ \rho \in \mathcal{I}\left(\widehat{\otimes}_{\pi_{s}}^{n, s} E ; F\right)$. De

$$
J_{i}(t \circ \rho)=t \circ \rho \circ i=t,
$$

concluímos que $J_{i}$ é sobrejetor.

Conforme já usamos anteriormente, o operador linearização

$$
L: \mathcal{I} \circ \mathcal{P}\left({ }^{n} E ; F\right) \longrightarrow \mathcal{I}\left(\widehat{\otimes}_{\pi_{s}}^{n, s} E ; F\right), L(P)=P_{L, s}
$$

é um isomorfismo isométrico (Proposições 1.6.14 e 1.6.16). Por ser a composição de operadores sobrejetores, o operador $J_{i} \circ L$

$$
\mathcal{I} \circ \mathcal{P}\left({ }^{n} E ; F\right) \stackrel{L}{\longrightarrow} \mathcal{I}\left(\widehat{\otimes}_{\pi_{s}}^{n, s} E ; F\right) \stackrel{J_{i}}{\longrightarrow} \mathcal{I}(E ; F)
$$

também é sobrejetor. E pelo Teorema 1.1.7(i) segue que seu adjunto $\left(J_{i} \circ L\right)^{*}=L^{*} \circ J_{i}^{*}$,

$$
[\mathcal{I}(E ; F)]^{*} \stackrel{J_{i}^{*}}{\longrightarrow}\left[\mathcal{I}\left(\widehat{\otimes}_{\pi_{s}}^{n, s} E ; F\right)\right]^{*} \stackrel{L^{*}}{\longrightarrow}\left[\mathcal{I} \circ \mathcal{P}\left({ }^{n} E ; F\right)\right]^{*}
$$

é um isomorfismo sobre sua imagem.

O próximo passo é provar que o diagrama

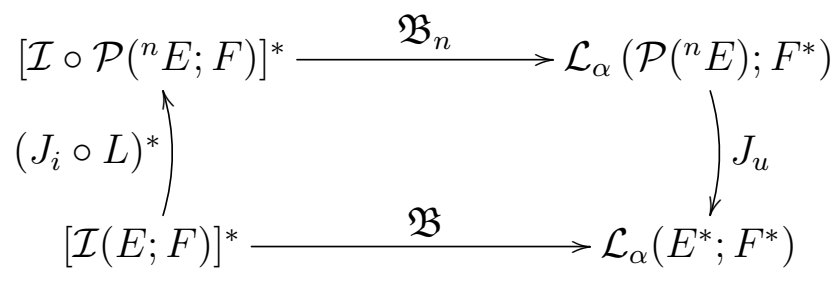

é comutativo. Primeiro, notemos que dados $x^{*} \in E^{*}$ e $y \in F$,

$$
\begin{aligned}
\left(\left(u\left(x^{*}\right)_{L, s} \otimes y\right) \circ i\right)(x) & =\left(u\left(x^{*}\right)_{L, s} \otimes y\right)(i(x)) \\
& =u\left(x^{*}\right)_{L, s}(i(x)) y \\
& =i^{*}\left(u\left(x^{*}\right)_{L, s}\right)(x) y \\
& =i^{*}\left(\ell\left(u\left(x^{*}\right)\right)(x) y\right. \\
& =\left(i^{*} \circ \ell \circ u\right)\left(x^{*}\right)(x) y \\
& =i d_{E^{*}}\left(x^{*}\right)(x) y=x^{*}(x) y=\left(x^{*} \otimes y\right)(x)
\end{aligned}
$$


para todo $x \in E$. Isso prova que $\left(u\left(x^{*}\right)_{L, s} \otimes y\right) \circ i=x^{*} \otimes y$.

Para $\phi \in[\mathcal{I}(E ; F)]^{*}, x^{*} \in E^{*}$ e $y \in F$,

$$
\begin{aligned}
\left(J_{u} \circ \mathfrak{B}_{n} \circ\left(J_{i} \circ L\right)^{*}\right)(\phi)\left(x^{*}\right)(y) & =\left(J_{u}\left(\mathfrak{B}_{n}\left(\left(J_{i} \circ L\right)^{*}(\phi)\right)\right)\right)\left(x^{*}\right)(y) \\
& =\left(\left(\mathfrak{B}_{n}\left(\left(J_{i} \circ L\right)^{*}(\phi)\right)\right) \circ u\right)\left(x^{*}\right)(y) \\
& =\mathfrak{B}_{n}\left(\left(J_{i} \circ L\right)^{*}(\phi)\right)\left(u\left(x^{*}\right)\right)(y) \\
& =\left(J_{i} \circ L\right)^{*}(\phi)\left(u\left(x^{*}\right) \otimes y\right) \\
& =\phi\left(\left(J_{i} \circ L\right)\left(u\left(x^{*}\right) \otimes y\right)\right) \\
& =\phi\left(J_{i}\left(L\left(u\left(x^{*}\right) \otimes y\right)\right)\right) \\
& =\phi\left(J_{i}\left(\left(u\left(x^{*}\right) \otimes y\right) L, s\right)\right) \\
& =\phi\left(J_{i}\left(u\left(x^{*}\right)_{L, s} \otimes y\right)\right) \\
& =\phi\left(\left(u\left(x^{*}\right)_{L, s} \otimes y\right) \circ i\right) \\
& =\phi\left(x^{*} \otimes y\right)=\mathfrak{B}(\phi)\left(x^{*}\right)(y) .
\end{aligned}
$$

Portanto, $\mathfrak{B}=J_{u} \circ \mathfrak{B}_{n} \circ\left(J_{i} \circ L\right)^{*}$, isto é, o diagrama comuta.

Sabemos que $X:=\left(J_{i} \circ L\right)^{*}\left(\mathcal{I}(E ; F)^{*}\right)$ é um subespaço fechado de $\left[\mathcal{I} \circ \mathcal{P}\left({ }^{n} E ; F\right)\right]^{*}$ e, denotando a restrição de $\left(J_{i} \circ L\right)^{*}$ ainda por $\left(J_{i} \circ L\right)^{*}$, também sabemos que

$$
\left(J_{i} \circ L\right)^{*}: \mathcal{I}(E ; F)^{*} \longrightarrow X
$$

é um isomorfismo. Para $P \in \mathcal{P}\left({ }^{n} E\right)$ e $y \in F$,

$$
\left(\left(P_{L} \otimes y\right) \circ i\right)(x)=\left(P_{L} \otimes y\right)(i(x))=P_{L}(i(x)) \otimes y=i^{*}\left(P_{L}\right)(x) \otimes y=\left(i^{*}\left(P_{L}\right) \otimes y\right)(x)
$$

para todo $x \in E$, provando que $\left(P_{L} \otimes y\right) \circ i=i^{*}\left(P_{L}\right) \otimes y$. Daí, dado $\phi \in[\mathcal{I}(E ; F)]^{*}$,

$$
\begin{aligned}
{\left[\mathfrak{B}_{n}\left(\left(J_{i} \circ L\right)^{*}(\phi)\right)\right](P)(y) } & =\left[\left(J_{i} \circ L\right)^{*}(\phi)\right](P \otimes y) \\
& =\phi\left(\left(J_{i} \circ L\right)(P \otimes y)\right) \\
& =\phi\left(J_{i}(L(P \otimes y))\right) \\
& =\phi\left(J_{i}\left((P \otimes y)_{L}\right)\right) \\
& =\phi\left(J_{i}\left(P_{L} \otimes y\right)\right) \\
& =\phi\left(\left(P_{L} \otimes y\right) \circ i\right) \\
& =\phi\left(i^{*}\left(P_{L}\right) \otimes y\right) \\
& =\mathfrak{B}(\phi)\left(i^{*}\left(P_{L}\right)\right)(y) \\
& =\left[\mathfrak{B}(\phi)\left(i^{*}(\ell(P))\right)\right](y)
\end{aligned}
$$




$$
\begin{aligned}
& =\left[\mathfrak{B}(\phi) \circ i^{*} \circ \ell\right](P)(y) \\
& =K(\mathfrak{B}(\phi))(P)(y)
\end{aligned}
$$

para todos $P \in \mathcal{P}\left({ }^{n} E\right)$ e $y \in F$, ou seja,

$$
\mathfrak{B}_{n}\left(\left(J_{i} \circ L\right)^{*}(\phi)\right)=K(\mathfrak{B}(\phi)) \in Y
$$

para todo $\phi \in[\mathcal{I}(E ; F)]^{*}$. Isso prova que $\mathfrak{B}_{n}(X)=\mathfrak{B}_{n}\left(\left(J_{i} \circ L\right)^{*}\left(\mathcal{I}(E ; F)^{*}\right)\right) \subseteq Y$. Como $Y$ é fechado e $\mathfrak{B}_{n}$ é um isomorfismo sobre sua imagem, $\mathfrak{B}_{n}(X)$ é um subespaço fechado de $Y$.

E como $J_{u}: Y \longrightarrow \mathcal{L}_{\alpha}\left(E^{*} ; F^{*}\right)$ é um isomorfismo, sua restrição a $\mathfrak{B}_{n}(X)$ é um isomorfismo sobre sua imagem. Dessa forma, denotando a restrição de $\mathfrak{B}_{n}$ a $X$ sobre sua imagem ainda por $\mathfrak{B}_{n}$ e a restrição de $J_{u}$ a $\mathfrak{B}_{n}(X)$ ainda por $J_{u}$, no diagrama comutativo

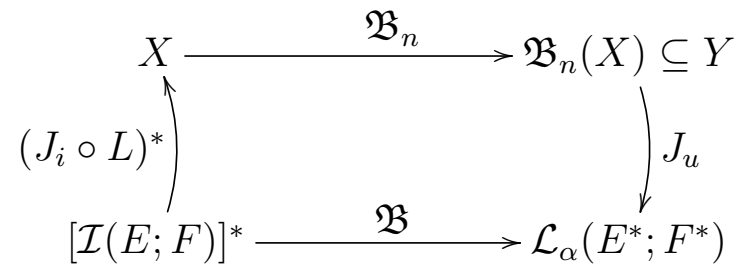

$\left(J_{i} \circ L\right)^{*}$ e $\mathfrak{B}_{n}$ são isomorfismos e $J_{u}$ é um isomorfismo sobre sua imagem. Segue que $\mathfrak{B}$ é um isomorfismo sobre sua imagem.

Observação 4.2.5. É importante mencionar que se, no teorema acima, supusermos que $\mathfrak{B}_{n}$ seja um isomorfismo isométrico, nem a demonstração acima e nem a demonstração correspondente do Teorema 3.2.1 garantem que $\mathfrak{B}$ é um isomorfismo isométrico. O ponto é que a sobrejetividade de $\mathfrak{B}_{n}$ não implica na sobrejetividade de $\mathfrak{B}$. Comparando o Teorema 3.2.1 com o teorema acima, vemos que, enquanto sabemos que a transformada de Borel linear é sobrejetora sempre que alguma hiper-transformada de Borel multilinear é um isomorfismo, não sabemos se a transformada de Borel linear é sobrejetora sempre que alguma hiper-transformada de Borel polinomial é um isomorfismo. Como mencionado antes, acreditamos que isso é mais uma manifestação das diferenças das teorias multilinear e polinomial. E o problema com a sobrejetividade é recorrente: relembre que, enquanto que o produto tensorial projetivo cheio $E_{1} \widehat{\otimes}_{\pi} \cdots \widehat{\otimes}_{\pi} E_{n}$, que é intimamente relacionado com operadores multilineares, é associativo (veja [68, Teorema 3.2.3]), o produto tensorial projetivo simétrico, que é intimamente relacionado com polinômios, não é associativo. Mais ainda, o problema - novamente - é a sobrejetividade: sabe-se que $\widehat{\otimes}_{\pi}^{m n, s} E$ é (canonicamente) isomorfo a um subespaço de $\widehat{\otimes}_{\pi}^{m, s}\left(\widehat{\otimes}_{\pi}^{n, s} E\right)$, mas não ao espaço todo (veja $[3])$. 


\subsection{Aplicações}

Analogamente ao caso multilinear, apresentaremos agora algumas representações concretas de funcionais lineares em espaços de polinômios por meio do Teorema 4.2.3.

\subsubsection{Polinômios homogêneos aproximáveis e compactos}

Como trabalhamos com ideais fechados nesta seção, é importante relembrar que, denotando por $P_{L, s}$ a linearização do polinômio $P$ no produto tensorial simétrico munido com a norma $s$-projetiva $\pi_{s}$, pelo Teorema 1.6.7 sabemos que $\|P\|=\left\|P_{L, s}\right\|$.

Seja $\overline{\mathcal{P}_{\mathcal{F}}}$ o fecho de $\mathcal{P}_{\mathcal{F}}$ na norma usual de polinômios, isto é, $\overline{\mathcal{P}_{\mathcal{F}}}$ é o hiper-ideal formado pelos polinômios homogêneos que podem ser aproximados, na norma usual, por polinômios de posto finito. Vejamos que funcionais lineares em $\overline{\mathcal{P}_{\mathcal{F}}}$ são representados por operadores lineares integrais.

Lema 4.3.1. Para todo $n \in \mathbb{N}$ e todos espaços de Banach $E$ e $F$,

$$
\overline{\mathcal{P}_{\mathcal{F}}}\left({ }^{n} E ; F\right)=\overline{\mathcal{F}} \circ \mathcal{P}\left({ }^{n} E ; F\right) .
$$

Demonstração. Para $P \in \mathcal{P}\left({ }^{n} E ; F\right)$,

$$
P \in \overline{\mathcal{P}_{\mathcal{F}}}\left({ }^{n} E ; F\right) \Longleftrightarrow P_{L} \in \overline{\mathcal{F}}\left(\widehat{\otimes}_{\pi_{s}}^{n, s} E ; F\right) \Longleftrightarrow P \in \overline{\mathcal{F}} \circ \mathcal{P}\left({ }^{n} E ; F\right),
$$

onde a primeira equivalência segue de [19, Theorem 2.2] e a segunda equivalência segue da já várias vezes usada Proposição 1.6.14.

Teorema 4.3.2. Para todos espaços de Banach E e F, a hiper-transformada de Borel polinomial

$$
\mathfrak{B}_{n}:\left[\overline{\mathcal{P}_{\mathcal{F}}}\left({ }^{n} E ; F\right)\right]^{*} \longrightarrow \mathcal{J}\left(\mathcal{P}\left({ }^{n} E\right) ; F^{*}\right), \mathfrak{B}_{n}(\phi)(P)(y)=\phi(P \otimes y),
$$

é um isomorfismo isométrico.

Demonstração. Na demonstração do Teorema 3.3.1 mostramos que a transformada de Borel linear

$\mathfrak{B}:[\overline{\mathcal{F}}(E ; F)]^{*} \longrightarrow \mathcal{J}\left(E^{*} ; F^{*}\right), \mathfrak{B}(\phi)\left(x^{*}\right)(y)=\phi\left(x^{*} \otimes y\right)$ 
é um isomorfismo isométrico. Como $\mathcal{J}$ é um ideal de Banach, portanto é um semi-ideal à esquerda, o Teorema 4.2.3 garante que a hiper-transformada de Borel polinomial

$$
\mathfrak{B}_{n}:\left[\overline{\mathcal{F}} \circ \mathcal{P}\left({ }^{n} E ; F\right)\right]^{*} \longrightarrow \mathcal{J}\left(\mathcal{P}\left({ }^{n} E\right) ; F^{*}\right),
$$

é um isomorfismo isométrico. O Lema 4.3 .1 completa a demonstração.

Veremos agora a representação de funcionais em espaços de polinômios homogêneos compactos como operadores lineares integrais, a qual, como no caso multilinear, é a primeira representação deste tipo que conhecemos.

Teorema 4.3.3. Se $\mathcal{P}\left({ }^{n} E\right)$ ou $F$ tem a propriedade da aproximação, então a hipertransformada de Borel polinomial

$$
\mathfrak{B}_{n}:\left[\mathcal{P}_{\mathcal{K}}\left({ }^{n} E ; F\right)\right]^{*} \longrightarrow \mathcal{J}\left(\mathcal{P}\left({ }^{n} E\right) ; F^{*}\right), \mathfrak{B}_{n}(\phi)(P)(y)=\phi(P \otimes y),
$$

é um isomorfismo isométrico.

Demonstração. Conforme mencionado na demonstração do Teorema 3.3.2, a transformada de Borel linear

$\mathfrak{B}:[\mathcal{K}(E ; F)]^{*} \longrightarrow \mathcal{J}\left(E^{*} ; F^{*}\right), \mathfrak{B}(\phi)\left(x^{*}\right)(y)=\phi\left(x^{*} \otimes y\right)$,

é um isomorfismo isométrico desde que $E^{*}$ ou $F$ possuam a propriedade da aproximação. Como $\mathcal{J}$ é ideal de Banach, em particular é um semi-ideal à esquerda, e a propriedade da aproximação é uma propriedade geométrica, pelo Teorema 4.2.3 segue que os espaços $\left[\mathcal{K} \circ \mathcal{P}\left({ }^{n} E ; F\right)\right]^{*}$ e $\mathcal{J}\left(\mathcal{P}\left({ }^{n} E\right) ; F^{*}\right)$ são isometricamente isomorfos por meio da hipertransformada de Borel, desde que $\mathcal{P}\left({ }^{n} E\right)$ ou $F$ possuam a propriedade da aproximação. A igualdade isométrica $\mathcal{P}_{\mathcal{K}}=\mathcal{K} \circ \mathcal{P}$ (veja Proposição 1.6.17) completa a demonstração.

\subsubsection{Polinômios homogêneos hiper-nucleares}

Representaremos nesta seção funcionais lineares em espaços de polinômios hiper- $(s ; r)$ nucleares, estudados em [72], como operadores lineares absolutamente somantes. Apesar das demonstrações funcionarem de forma análoga ao caso de operadores multilineares, optamos por apresentá-las na íntegra, pois, conforme já experimentamos nesta própria tese, nem sempre o caso polinomial funciona como o caso multilinear. Por isso julgamos ser justificada a apresentação das demonstrações do caso polinomial. 
Relembre a definição dos hiper-ideais dos polinômios homogêneos hiper- $(s ; r)$-nucleares na Definição 4.1.5.

Conforme mencionado anteriormente, Torres [72, Teorema 3.2.10] provou que $\mathcal{P}_{\mathcal{H} \mathcal{N}_{(s ; r)}}$ é um hiper-ideal de Banach de polinômios. Em particular vale a desigualdade que $\|\cdot\| \leq$ $\|\cdot\|_{\mathcal{P}_{\mathcal{H N}}(s ; r)}$.

Proposição 4.3.4. Sejam E, F espaços de Banach e $n \in \mathbb{N}$. Então,

$$
\mathcal{P}_{\mathcal{H N}(s ; r)}\left({ }^{n} E ; F\right)=\mathcal{N}_{(s ; r)} \circ \mathcal{P}\left({ }^{n} E ; F\right)
$$

$\operatorname{com}\|\cdot\|_{\mathcal{P}_{\mathcal{H N}}(s ; r)}=\|\cdot\|_{\mathcal{N}_{(s ; r)} \circ \mathcal{P}}$.

Demonstração. Seja $P \in \mathcal{N}_{(s ; r)} \circ \mathcal{P}\left({ }^{n} E ; F\right)$. Existem um espaço de Banach $G$, um polinômio $n$-homogêneo $Q \in \mathcal{P}\left({ }^{n} E ; G\right)$ e um operador $u \in \mathcal{N}_{(s ; r)}(G ; F)$ tais que $P=u \circ Q$.

Dado $\varepsilon>0$, como $u \in \mathcal{N}_{(s ; r)}(G ; F)$, podemos tomar $\left(\lambda_{j}\right)_{j=1}^{\infty} \in \ell_{s},\left(y_{j}\right)_{j=1}^{\infty} \in \ell_{\infty}(F)$ e $\left(\varphi_{j}\right)_{j=1}^{\infty} \in \ell_{r}^{w}\left(G^{*}\right)$ tais que $u=\sum_{j=1}^{\infty} \lambda_{j} \varphi_{j} \otimes y_{j} \mathrm{e}$

$$
\left\|\left(\lambda_{j}\right)_{j=1}^{\infty}\right\|_{s} \cdot\left\|\left(\varphi_{j}\right)_{j=1}^{\infty}\right\|_{w, r} \cdot\left\|\left(y_{j}\right)_{j=1}^{\infty}\right\|_{\infty} \leq(1+\varepsilon)\|u\|_{\mathcal{N}_{(s ; r)}} .
$$

De $P=u \circ Q$ segue que

$$
P(x)=u(Q(x))=\sum_{j=1}^{\infty} \lambda_{j} \varphi_{j}(Q(x)) y_{j}=\sum_{j=1}^{\infty} \lambda_{j}\left(\varphi_{j} \circ Q\right) \otimes y_{j}(x),
$$

para qualquer $x \in E$. Mostremos que $P=\sum_{j=1}^{\infty} \lambda_{j}\left(\varphi_{j} \circ Q\right) \otimes y_{j}$ é uma representação hiper- $(s ; r)$-nuclear de $P$. De fato, já sabemos que $\left(\lambda_{j}\right)_{j=1}^{\infty} \in \ell_{s}$ e $\left(y_{j}\right)_{j=1}^{\infty} \in \ell_{\infty}(F)$, e resta verificar que $\left(\varphi_{j} \circ Q\right)_{j=1}^{\infty} \in \ell_{r}^{w}\left(\mathcal{P}\left({ }^{n} E\right)\right)$. Consideremos o adjunto de Aron-Schottenloher do polinômio $Q$ :

$$
Q^{*}: G^{*} \longrightarrow \mathcal{P}\left({ }^{n} E\right), Q^{*}(\psi)(x)=\psi(Q(x)) .
$$

Pela Proposição 1.6.10 sabemos que $Q^{*}$ está bem definido, é linear e $\left\|Q^{*}\right\|=\|Q\|$.

Do Lema 1.1.9, segue que

$$
\left(\varphi_{j} \circ Q\right)_{j=1}^{\infty}=\left(Q^{*}\left(\varphi_{j}\right)\right)_{j=1}^{\infty} \in \ell_{r}^{w}\left(\mathcal{P}\left({ }^{n} E\right)\right)
$$

e

$$
\left\|\left(\varphi_{j} \circ Q\right)_{j=1}^{\infty}\right\|_{w, r}=\left\|\left(Q^{*}\left(\varphi_{j}\right)\right)_{j=1}^{\infty}\right\|_{w, r} \leq\left\|Q^{*}\right\| \cdot\left\|\left(\varphi_{j}\right)_{j=1}^{\infty}\right\|_{w, r}=\|Q\| \cdot\left\|\left(\varphi_{j}\right)_{j=1}^{\infty}\right\|_{w, r} .
$$


Portanto, $P=\sum_{j=1}^{\infty} \lambda_{j}\left(\varphi_{j} \circ Q\right) \otimes y_{j} \in \mathcal{P}_{\mathcal{H N}_{(s ; r)}}(E ; F) \mathrm{e}$

$$
\begin{aligned}
\|P\|_{\mathcal{P}_{\mathcal{H N}}(s ; r)} & \leq\left\|\left(\lambda_{j}\right)_{j=1}^{\infty}\right\|_{s} \cdot\left\|\left(\varphi_{j} \circ Q\right)_{j=1}^{\infty}\right\|_{w, r} \cdot\left\|\left(y_{j}\right)_{j=1}^{\infty}\right\|_{\infty} \\
& \leq\left\|\left(\lambda_{j}\right)_{j=1}^{\infty}\right\|_{s} \cdot\left\|\left(\varphi_{j}\right)_{j=1}^{\infty}\right\|_{w, r} \cdot\|Q\| \cdot\left\|\left(y_{j}\right)_{j=1}^{\infty}\right\|_{\infty} \\
& \leq\|Q\| \cdot(1+\varepsilon) \cdot\|u\|_{\mathcal{N}_{(s ; r)}} .
\end{aligned}
$$

Fazendo $\varepsilon \longrightarrow 0^{+}$, obtemos $\|P\|_{\mathcal{P}_{\mathcal{H N}_{(s ; r)}}} \leq\|Q\| \cdot\|u\|_{\mathcal{N}_{(s ; r)}}$ para toda fatoração $P=u \circ Q$, com $u \in \mathcal{N}_{(s ; r)}(G ; F)$. Tomando o ínfimo sobre todas essas fatorações segue que

$$
\|P\|_{\mathcal{P}_{\mathcal{H N}}(s ; r)} \leq\|P\|_{\mathcal{N}_{(s ; r)} \circ \mathcal{P}}
$$

Reciprocamente, dados $P \in \mathcal{P}_{\mathcal{H N}_{(s ; r)}}\left({ }^{n} E ; F\right)$ e $\varepsilon>0$, existem $\left(\lambda_{j}\right)_{j=1}^{\infty} \in \ell_{s},\left(P_{j}\right)_{j=1}^{\infty} \in$ $\ell_{r}^{w}\left(\mathcal{P}\left({ }^{n} E\right)\right)$ e $\left(y_{j}\right)_{j=1}^{\infty} \in \ell_{\infty}(F)$ tais que

$$
P(x)=\sum_{j=1}^{\infty} \lambda_{j} P_{j}(x) y_{j}
$$

para todo $x \in E$ e

$$
\left\|\left(\lambda_{j}\right)_{j=1}^{\infty}\right\|_{s} \cdot\left\|\left(P_{j}\right)_{j=1}^{\infty}\right\|_{w, r} \cdot\left\|\left(y_{j}\right)_{j=1}^{\infty}\right\|_{\infty} \leq(1+\varepsilon)\|P\|_{\mathcal{P}_{\mathcal{H N}}(s ; r)}
$$

Pela Proposição 1.6.14, é suficiente mostrarmos que $P_{L, s} \in \mathcal{N}_{(s ; r)}\left(\widehat{\otimes}_{\pi_{s}}^{n, s} E ; F\right)$. Pela Proposição 4.1.8, sabemos que a convergência $P=\sum_{j=1}^{\infty} \lambda_{j} P_{j} \otimes y_{j}$ se dá na norma $\|\cdot\|_{\mathcal{P}_{\mathcal{H N}}(s ; r)}$. Da desigualdade $\|\cdot\| \leq\|\cdot\|_{\mathcal{P}_{\mathcal{H N}}(s ; r)}$ concluímos que $P=\sum_{j=1}^{\infty} \lambda_{j} P_{j} \otimes y_{j}$ na norma usual $\|\cdot\|$ e podemos escrever

$$
P=\lim _{m \rightarrow \infty} \sum_{j=1}^{m} \lambda_{j} P_{j} \otimes y_{j} \quad \text { em } \mathcal{P}\left({ }^{n} E ; F\right)
$$

Usando o fato de que o operador linearização $L: \mathcal{P}\left({ }^{n} E\right) \longrightarrow\left(\widehat{\otimes}_{\pi_{s}}^{n, s} E\right)^{*}, L(P)=P_{L, s}$ é um isomorfismo isométrico, temos

$$
P_{L, s}=\left(\lim _{m} \sum_{j=1}^{m} \lambda_{j} P_{j} \otimes y_{j}\right)_{L, s}=\lim _{m \rightarrow \infty} \sum_{j=1}^{m} \lambda_{j}\left(P_{j} \otimes y_{j}\right)_{L, s}=\sum_{j=1}^{\infty} \lambda_{j}\left(P_{j}\right)_{L, s} \otimes y_{j} .
$$

Mostremos que (4.9) é uma representação nuclear de $P_{L, s}$ : Com efeito, $\left(\lambda_{j}\right)_{j=1}^{\infty} \in \ell_{s}$, $\left(y_{j}\right)_{j=1}^{\infty} \in \ell_{\infty}(F)$, e pela Proposição 1.23 sabemos que

$$
\left(\left(P_{j}\right)_{L, s}\right)_{j=1}^{\infty}=\left(L\left(P_{j}\right)\right)_{j=1}^{\infty} \in \ell_{r}^{w}\left(\left(\widehat{\otimes}_{\pi}^{n, s} E\right)^{*}\right)
$$


e

$$
\left\|\left(\left(P_{j}\right)_{L, s}\right)_{j=1}^{\infty}\right\|_{w, r}=\left\|\left(L\left(P_{j}\right)\right)_{j=1}^{\infty}\right\|_{w, r} \leq\|L\| \cdot\left\|\left(P_{j}\right)_{j=1}^{\infty}\right\|_{w, r}=\left\|\left(P_{j}\right)_{j=1}^{\infty}\right\|_{w, r} .
$$

Daí,

$$
\begin{aligned}
\left\|P_{L, s}\right\|_{\mathcal{N}_{(s ; r)}} & \leq\left\|\left(\lambda_{j}\right)_{j=1}^{\infty}\right\|_{s} \cdot\left\|\left(\left(P_{j}\right)_{L, s}\right)_{j=1}^{\infty}\right\|_{w, r} \cdot\left\|\left(y_{j}\right)_{j=1}^{\infty}\right\|_{\infty} \\
& \leq\left\|\left(\lambda_{j}\right)_{j=1}^{\infty}\right\|_{s} \cdot\left\|\left(P_{j}\right)_{j=1}^{\infty}\right\|_{w, r} \cdot\left\|\left(y_{j}\right)_{j=1}^{\infty}\right\|_{\infty} \\
& \leq(1+\varepsilon)\|P\|_{\mathcal{P}_{\mathcal{H N}}(s ; r)} .
\end{aligned}
$$

Fazendo $\varepsilon \longrightarrow 0^{+}$e aplicando a Proposição 1.6.16 concluímos que

$$
\|P\|_{\mathcal{N}_{(s ; r)} \circ \mathcal{P}}=\left\|P_{L, s}\right\|_{\mathcal{N}_{(s ; r)}} \leq\|P\|_{\mathcal{P}_{\mathcal{H N}}(s ; r)}
$$

Relembre que no caso $s=1$ e $r=+\infty$, chamamos os polinômios homogêneos hiper$(1 ;+\infty)$-nucleares simplesmente de hiper-nucleares e que o hiper-ideal de polinômios correspondente é denotado por $\left(\mathcal{P}_{\mathcal{H N}},\|\cdot\|_{\mathcal{P}_{\mathcal{H N}}}\right)$. Relembre também que estamos denotando o ideal de Banach dos operadores lineares nucleares por $\left(\mathcal{N},\|\cdot\|_{\mathcal{N}}\right)$.

Corolário 4.3.5. Sejam E, $F$ espaços de Banach e $n \in \mathbb{N}$. Então,

$$
\mathcal{P}_{\mathcal{H N}}\left({ }^{n} E ; F\right)=\mathcal{N} \circ \mathcal{P}\left({ }^{n} E ; F\right)
$$

$\operatorname{com}\|\cdot\|_{\mathcal{P}_{\mathcal{H N}}}=\|\cdot\|_{\mathcal{N} \circ \mathcal{P}}$.

Conforme sugerido pelo Teorema 4.1.9, vejamos que de fato a hiper-transformada de Borel polinomial representa funcionais lineares em espaços de polinômios hiper-nucleares como operadores de $\mathcal{L}\left(\mathcal{P}\left({ }^{n} E\right) ; F^{*}\right)$ com a norma usual.

Teorema 4.3.6. Se $\mathcal{P}\left({ }^{n} E\right)$ ou $F$ tem a propriedade da aproximação limitada, então a hiper-transformada de Borel polinomial

$$
\mathfrak{B}_{n}:\left[\mathcal{P}_{\mathcal{H N}}\left({ }^{n} E ; F\right)\right]^{*} \longrightarrow \mathcal{L}\left(\mathcal{P}\left({ }^{n} E\right) ; F^{*}\right), \mathfrak{B}_{n}(\phi)(P)(y)=\phi(P \otimes y),
$$

é um isomorfismo isométrico.

Demonstração. Pela Proposição 3.3.3 sabemos que a transformada de Borel linear

$$
\mathfrak{B}:[\mathcal{N}(E ; F)]^{*} \longrightarrow \mathcal{L}\left(E^{*} ; F^{*}\right), \mathfrak{B}(\phi)\left(x^{*}\right)(y)=\phi\left(x^{*} \otimes y\right),
$$

é um isomorfismo isométrico desde que $E^{*}$ ou $F$ tenha a propriedade da aproximação limitada. Como a propriedade da aproximação limitada é uma propriedade geométrica, o Teorema 4.2.3 e o Corolário 4.3.5 concluem a demonstração. 
Terminamos esta seção representando funcionais lineares em espaços de polinômios hiper- $(s ; r)$-nucleares como operadores lineares absolutamente somantes.

Teorema 4.3.7. Suponha que $\mathcal{P}\left({ }^{n} E\right)$ tenha a propriedade da aproximação limitada. Então, para todo espaço de Banach F, a hiper-transformada de Borel

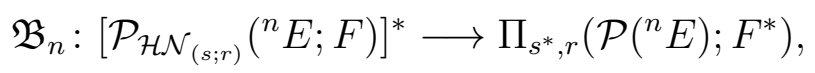

é um isomorfismo isométrico.

Demonstração. O caso linear do Teorema 3.0.2 afirma que se $E^{*}$ tem a propriedade da aproximação limitada, então a transformada de Borel linear

$$
\mathfrak{B}:\left[\mathcal{N}_{(s ; r)}(E ; F)\right]^{*} \longrightarrow \Pi_{s^{*}, r}\left(E^{*} ; F^{*}\right),
$$

é um isomorfismo isométrico. Como $\Pi_{s^{*}, r}$ é um ideal de Banach (Exemplo 1.4.6), e portanto um semi-ideal à esquerda, combinando o Teorema 4.2.3 com a Proposição 4.3.4 obtemos a representação desejada.

\subsubsection{Polinômios homogêneos hiper- $\sigma(p)$-nucleares}

Estudaremos nesta seção a contrapartida polinomial da classe de operadores multilineares introduzida na Seção 2.2. E, a exemplo do que fizemos na Subseção 3.3.3, representaremos funcionais lineares em espaços de polinômios hiper- $\sigma(p)$-nucleares como operadores lineares quase- $\tau(p)$-somantes.

De forma natural, os espaços de polinômios homogêneos a valores escalares desempenharão o papel que é dos espaços de formas no caso multilinear.

Definição 4.3.8. Seja $1 \leq p<\infty$. Dizemos que um polinômio $n$-homogêneo $P \in$ $\mathcal{P}\left({ }^{n} E ; F\right)$ é hiper- $\sigma(p)$-nuclear se existem sequências $\left(\lambda_{j}\right)_{j=1}^{\infty} \in \ell_{p^{*}},\left(P_{j}\right)_{j=1}^{\infty}$ em $\mathcal{P}\left({ }^{n} E\right)$ e $\left(y_{j}\right)_{j=1}^{\infty}$ em $F$ tais que

$$
\begin{gathered}
P(x)=\sum_{j=1}^{\infty} \lambda_{j} P_{j}(x) y_{j} \text { para todo } x \in E \\
\sup _{x \in B_{E}, y^{*} \in B_{F^{*}}}\left(\sum_{j=1}^{\infty}\left|P_{j}(x) y^{*}\left(y_{j}\right)\right|^{p}\right)^{\frac{1}{p}}<\infty \mathrm{e} \\
\lim _{m \rightarrow \infty} \sup _{x \in B_{E}, y^{*} \in B_{F^{*}}}\left(\sum_{j=m}^{\infty}\left|P_{j}(x) y^{*}\left(y_{j}\right)\right|^{p}\right)^{\frac{1}{p}}=0 .
\end{gathered}
$$


Neste caso dizemos que $P=\sum_{j=1}^{\infty} \lambda_{j} P_{j} \otimes y_{j}$ é uma representação hiper- $\sigma(p)$-nuclear de $P$, escrevemos $P \in \mathcal{P}_{\mathcal{H N}_{\sigma(p)}}\left({ }^{n} E ; F\right)$ e definimos

$$
\|P\|_{\mathcal{P}_{\mathcal{H N} \sigma(p)}}=\inf \left\{\left\|\left(\lambda_{j}\right)_{j=1}^{\infty}\right\|_{p^{*}} \cdot \sup _{x \in B_{E}, y^{*} \in B_{F^{*}}}\left(\sum_{j=1}^{\infty}\left|P_{j}(x) y^{*}\left(y_{j}\right)\right|^{p}\right)^{\frac{1}{p}}\right\},
$$

onde o ínfimo é tomado sobre todas as representações hiper- $\sigma(p)$-nucleares de $P$.

O caso linear $n=1$ recupera os operadores lineares $\sigma(p)$-nucleares de [15] e o caso $n=p=1$ recupera os operadores lineares $\sigma$-nucleares de [59].

Vejamos que $\mathcal{P}_{\mathcal{H N}}$ (p) é um ideal de composição.

Proposição 4.3.9. Sejam $1 \leq p<\infty, E$ e F espaços de Banach. Então

$$
\mathcal{P}_{\mathcal{H N} \sigma(p)}\left({ }^{n} E ; F\right)=\mathcal{N}_{\sigma(p)} \circ \mathcal{P}\left({ }^{n} E ; F\right)
$$

$e\|\cdot\|_{\mathcal{P}_{\mathcal{H}}}=\|\cdot\|_{\mathcal{N}_{\sigma(p)} \circ \mathcal{P}} \cdot$

Demonstração. Seja $P \in \mathcal{N}_{\sigma(p)} \circ \mathcal{P}\left({ }^{n} E ; F\right)$. Então existem um espaço de Banach $G$, $Q \in \mathcal{P}\left({ }^{n} E ; G\right)$ e $u \in \mathcal{N}_{\sigma(p)}(G ; F)$ tais que $P=u \circ Q$.

Dado $\varepsilon>0$, como $u \in \mathcal{N}_{\sigma(p)}(G ; F)$, podemos tomar sequências $\left(\lambda_{j}\right)_{j=1}^{\infty} \in \ell_{p^{*}},\left(\varphi_{j}\right)_{j=1}^{\infty} \subseteq$ $G^{*}$ e $\left(y_{j}\right)_{j=1}^{\infty} \subseteq F$ tais que

$$
\begin{gathered}
u(z)=\sum_{j=1}^{\infty} \lambda_{j} \varphi_{j}(z) y_{j} \text { para todo } z \in G \\
\sup _{z \in B_{G}, y^{*} \in B_{F^{*}}}\left(\sum_{j=1}^{\infty}\left|\varphi_{j}(z) y^{*}\left(y_{j}\right)\right|^{p}\right)^{\frac{1}{p}}<\infty, \lim _{m \rightarrow \infty} \sup _{z \in B_{G}, y^{*} \in B_{F^{*}}}\left(\sum_{j=m}^{\infty}\left|\varphi_{j}(z) y^{*}\left(y_{j}\right)\right|^{p}\right)^{\frac{1}{p}}=0 \mathrm{e} \\
\left\|\left(\lambda_{j}\right)_{j=1}^{\infty}\right\|_{p^{*}} \cdot \sup _{z \in B_{G}, y^{*} \in B_{F^{*}}}\left(\sum_{j=1}^{\infty}\left|\varphi_{j}(z) y^{*}\left(y_{j}\right)\right|^{p}\right)^{\frac{1}{p}} \leq(1+\varepsilon)\|u\|_{\mathcal{N}_{\sigma(p)} .}
\end{gathered}
$$

De $P=u \circ Q$ obtemos que

$$
P(x)=(u \circ Q)(x)=u(Q(x))=\sum_{j=1}^{\infty} \lambda_{j} \varphi_{j}(Q(x)) y_{j}=\sum_{j=1}^{\infty} \lambda_{j}\left(\varphi_{j} \circ Q\right) \otimes y_{j}(x)
$$


para todo $x \in E$. Verifiquemos que $P=\sum_{j=1}^{\infty} \lambda_{j}\left(\varphi_{j} \circ Q\right) \otimes y_{j}$ é uma representação hiper$\sigma(p)$-nuclear de $P$. Com efeito, já sabemos que $\left(\lambda_{j}\right)_{j=1}^{\infty} \in \ell_{p^{*}},\left(\varphi_{j} \circ Q\right)_{j=1}^{\infty} \subseteq \mathcal{P}\left({ }^{n} E\right)$ e $\left(y_{j}\right)_{j=1}^{\infty} \subseteq F$. Temos também

$$
\begin{gathered}
\left.\sup _{x \in B_{E}, y^{*} \in B_{F^{*}}}\left(\sum_{j=1}^{\infty}\left|\left(\varphi_{j} \circ Q\right)(x) y^{*}\left(y_{j}\right)\right|^{p}\right)^{\frac{1}{p}}=\left.\sup _{x \in B_{E}, y^{*} \in B_{F^{*}}}\left(\sum_{j=1}^{\infty} \mid \varphi_{j}(Q(x)) y^{*}\left(y_{j}\right)\right)\right|^{p}\right)^{\frac{1}{p}} \\
(Q \neq 0)=\|Q\| \cdot \sup _{x \in B_{E}, y^{*} \in B_{F^{*}}}\left(\sum_{j=1}^{\infty}\left|\varphi_{j}\left[\left(\frac{Q}{\|Q\|}\right)(x)\right] y^{*}\left(y_{j}\right)\right|^{p}\right)^{\frac{1}{p}} \\
\leq\|Q\| \cdot \sup _{z \in B_{G}, y^{*} \in B_{F^{*}}}\left(\sum_{j=1}^{\infty}\left|\varphi_{j}(z) y^{*}\left(y_{j}\right)\right|^{p}\right)^{\frac{1}{p}}<\infty
\end{gathered}
$$

pois $u \in \mathcal{N}_{\sigma(p)}(G ; F)$. Analogamente,

$$
\begin{aligned}
\lim _{m \rightarrow \infty} \sup _{x \in B_{E}, y^{*} \in B_{F^{*}}}( & \left(\sum_{j=m}^{\infty}\left|\left(\varphi_{j} \circ Q\right)(x) y^{*}\left(y_{j}\right)\right|^{p}\right)^{\frac{1}{p}} \\
& \leq \lim _{m \rightarrow \infty}\|Q\| \cdot \sup _{z \in B_{G}, y^{*} \in B_{F^{*}}}\left(\sum_{j=m}^{\infty}\left|\varphi_{j}(z) y^{*}\left(y_{j}\right)\right|^{p}\right)^{\frac{1}{p}} \\
& =\|Q\| \cdot \lim _{m \rightarrow \infty} \sup _{z \in B_{G}, y^{*} \in B_{F^{*}}}\left(\sum_{j=m}^{\infty}\left|\varphi_{j}(z) y^{*}\left(y_{j}\right)\right|^{p}\right)^{\frac{1}{p}}=0 .
\end{aligned}
$$

Portanto, $P=\sum_{j=1}^{\infty} \lambda_{j}\left(\varphi_{j} \circ Q\right) \otimes y_{j} \in \mathcal{P}_{\mathcal{H N} \sigma(p)}\left({ }^{n} E ; F\right)$ e

$$
\begin{aligned}
\|P\|_{\mathcal{P}_{\mathcal{H N}(p)}} & \leq\left\|\left(\lambda_{j}\right)_{j=1}^{\infty}\right\|_{p^{*}} \cdot \sup _{x \in B_{E}, y^{*} \in B_{F^{*}}}\left(\sum_{j=1}^{\infty}\left|\left(\varphi_{j} \circ Q\right)(x) y^{*}\left(y_{j}\right)\right|^{p}\right)^{\frac{1}{p}} \\
& (Q \neq 0)=\|Q\| \cdot\left\|\left(\lambda_{j}\right)_{j=1}^{\infty}\right\|_{p^{*}} \cdot \sup _{x \in B_{E}, y^{*} \in B_{F^{*}}}\left(\sum_{j=1}^{\infty}\left|\varphi_{j}\left[\left(\frac{Q}{\|Q\|}\right)(x)\right] y^{*}\left(y_{j}\right)\right|^{p}\right)^{\frac{1}{p}} \\
& \leq\|Q\| \cdot\left\|\left(\lambda_{j}\right)_{j=1}^{\infty}\right\|_{p^{*}} \cdot \sup _{z \in B_{G}, y^{*} \in B_{F^{*}}}\left(\sum_{j=1}^{\infty}\left|\varphi_{j}(z) y^{*}\left(y_{j}\right)\right|^{p}\right)^{\frac{1}{p}} \\
& \leq(1+\varepsilon)\|Q\| \cdot\|u\|_{\mathcal{N}_{\sigma(p)}} .
\end{aligned}
$$

Fazendo $\varepsilon \longrightarrow 0^{+}$, obtemos que $\|P\|_{\mathcal{P}_{\mathcal{H N} \sigma(p)}} \leq\|Q\| \cdot\|u\|_{\mathcal{N}_{\sigma(p)}}$ para toda fatoração $P=u \circ Q$ com $u \in \mathcal{N}_{\sigma(p)}$. Tomando o ínfimo sobre todas essas fatorações concluímos que $\|P\|_{\mathcal{P}_{\mathcal{H N} \sigma(p)}} \leq\|P\|_{\mathcal{N}_{\sigma(p)} \circ \mathcal{P} \text {. }}$ 
Reciprocamente, seja $P \in \mathcal{P}_{\mathcal{H N} \sigma(p)}\left({ }^{n} E ; F\right)$. Aplicando uma vez mais a Proposição 1.6.16, existe um único operador linear $P_{L, s} \in \mathcal{P}\left(\widehat{\otimes}_{\pi_{s}}^{n, s} E ; F\right)$ tal que $P=P_{L, s} \circ \rho_{n}$ e é suficiente mostrarmos que $P_{L, s} \in \mathcal{N}_{\sigma(p)}\left(\widehat{\otimes}_{\pi_{s}}^{n, s} E ; F\right)$.

Dado $\varepsilon>0$, tomemos $P=\sum_{j=1}^{\infty} \lambda_{j} P_{j} \otimes y_{j}$ uma representação hiper- $\sigma(p)$-nuclear de $P$, isto é, $\left(\lambda_{j}\right)_{j=1}^{\infty} \in \ell_{p^{*}},\left(P_{j}\right)_{j=1}^{\infty} \subseteq \mathcal{P}\left({ }^{n} E\right)$ e $\left(y_{j}\right)_{j=1}^{\infty} \subseteq F$ são tais que

$$
\begin{gathered}
\sup _{x \in B_{E}, y^{*} \in B_{F^{*}}}\left(\sum_{j=1}^{\infty}\left|P_{j}(x) y^{*}\left(y_{j}\right)\right|^{p}\right)^{\frac{1}{p}}<\infty \\
\lim _{m \rightarrow \infty} \sup _{x \in B_{E}, y^{*} \in B_{F^{*}}}\left(\sum_{j=m}^{\infty}\left|P_{j}(x) y^{*}\left(y_{j}\right)\right|^{p}\right)^{\frac{1}{p}}=0 \mathrm{e} \\
\left\|\left(\lambda_{j}\right)_{j=1}^{\infty}\right\|_{p^{*}} \cdot \sup _{x \in B_{E}, y^{*} \in B_{F^{*}}}\left(\sum_{j=1}^{\infty}\left|P_{j}(x) y^{*}\left(y_{j}\right)\right|^{p}\right)^{\frac{1}{p}} \leq(1+\varepsilon)\|P\|_{\mathcal{P}_{\mathcal{H N} \sigma(p)}} .
\end{gathered}
$$

No lugar de provar versões polinomiais das Proposições 2.2.2 e 2.2.3, provemos diretamente que a convergência $P=\sum_{j=1}^{\infty} \lambda_{j} P_{j} \otimes y_{j}$ se dá na norma usual:

$$
\begin{aligned}
& \left\|P-\sum_{j=1}^{k} \lambda_{j} P_{j} \otimes y_{j}\right\|=\sup _{x \in B_{E}}\left\|\sum_{j=1}^{\infty} \lambda_{j} P_{j}(x) y_{j}-\sum_{j=1}^{k} \lambda_{j} P_{j}(x) y_{j}\right\| \\
& =\sup _{x \in B_{E}} \sup _{y^{*} \in B_{F^{*}}}\left|y^{*}\left(\sum_{j=k+1}^{\infty} \lambda_{j} P_{j}(x) y_{j}\right)\right| \\
& =\sup _{x \in B_{E}, y^{*} \in B_{F^{*}}}\left|\sum_{j=k+1}^{\infty} \lambda_{j} P_{j}(x) y^{*}\left(y_{j}\right)\right| \\
& \leq \sup _{x \in B_{E}, y^{*} \in B_{F^{*}}}\left(\sum_{j=k+1}^{\infty}\left|\lambda_{j}\right|^{p^{*}}\right)^{\frac{1}{p^{*}}} \cdot\left(\sum_{j=k+1}^{\infty}\left|P_{j}(x) y^{*}\left(y_{j}\right)\right|^{p}\right)^{\frac{1}{p}} \\
& \leq\left(\sum_{j=k+1}^{\infty}\left|\lambda_{j}\right|^{p^{*}}\right)^{\frac{1}{p^{*}}} \cdot \sup _{x \in B_{E}, y^{*} \in B_{F^{*}}} \cdot\left(\sum_{j=1}^{\infty}\left|P_{j}(x) y^{*}\left(y_{j}\right)\right|^{p}\right)^{\frac{1}{p}} \stackrel{k \rightarrow \infty}{\longrightarrow} 0
\end{aligned}
$$

por $(4.10)$ e por $\left(\lambda_{j}\right)_{j=1}^{\infty}$ ser um elemento de $\ell_{p^{*}}$.

Usando que a correspondência

$$
P \in \mathcal{P}\left({ }^{n} E ; F\right) \mapsto P_{L, s} \in \mathcal{L}\left(\widehat{\otimes}_{\pi_{s}}^{n, s} E ; F\right)
$$


é um isomorfismo isométrico (Teorema 1.6.7) e aplicando o Lema 4.2.2, temos

$$
\begin{aligned}
P_{L, s} & =\left(\sum_{j=1}^{\infty} \lambda_{j} P_{j} \otimes y_{j}\right)_{L, s}=\left(\lim _{m} \sum_{j=1}^{m} \lambda_{j} P_{j} \otimes y_{j}\right)_{L, s}=\lim _{m}\left(\sum_{j=1}^{m} \lambda_{j} P_{j} \otimes y_{j}\right)_{L, s} \\
& =\lim _{m} \sum_{j=1}^{m} \lambda_{j}\left(P_{j} \otimes y_{j}\right)_{L, s}=\lim _{m} \sum_{j=1}^{m} \lambda_{j}\left(P_{j}\right)_{L, s} \otimes y_{j}=\sum_{j=1}^{\infty} \lambda_{j}\left(P_{j}\right)_{L, s} \otimes y_{j}
\end{aligned}
$$

na norma de $\mathcal{L}\left(\widehat{\otimes}_{\pi_{s}}^{n, s} E ; F\right)$.

Vejamos que $\sum_{j=1}^{\infty} \lambda_{j}\left(P_{j}\right)_{L, s} \otimes y_{j}$ é uma representação hiper- $\sigma(p)$-nuclear de $P_{L, s}$. Mesmo sendo análogo ao caso multilinear, faremos os detalhes. Dados escalares $\lambda_{1}, \ldots, \lambda_{m}$, segue da dualidade $\left(\ell_{p}^{m}\right)^{*}=\ell_{p^{*}}^{m}$ e do Teorema de Hahn-Banach que existem escalares $\theta_{1}, \ldots, \theta_{m}$ tais que

$$
\left\|\left(\theta_{j}\right)_{j=1}^{m}\right\|_{p^{*}}=1 \text { e }\left(\sum_{j=1}^{m}\left|\lambda_{j}\right|^{p}\right)^{1 / p}=\left|\sum_{j=1}^{m} \theta_{j} \lambda_{j}\right| .
$$

Voltando à demonstração, dados $k \geq m$, usando uma vez mais que a norma de um polinômio homogêneo coincide com a norma simétrica projetiva da sua linearização, temos

$$
\left\|\sum_{j=m}^{k} P_{j} \otimes y_{j}\right\|=\left\|\left(\sum_{j=m}^{k} P_{j} \otimes y_{j}\right)_{L, s}\right\|=\left\|\sum_{j=m}^{k}\left(P_{j}\right)_{L, s} \otimes y_{j}\right\| .
$$

Assim, para cada $z \in B_{\widehat{\otimes}_{\pi_{s}}^{n, s} E}$, cada $y^{*} \in B_{F^{*}}$ e cada $j=m, \ldots, k$, existem escalares $\theta_{m}, \ldots, \theta_{k}$ tais que

$$
\left\|\left(\theta_{j}\right)_{j=m}^{k}\right\|_{p^{*}}=1 \text { e }\left(\sum_{j=m}^{k}\left|\left(P_{j}\right)_{L, s}(z) y^{*}\left(y_{j}\right)\right|^{p}\right)^{1 / p}=\left|\sum_{j=m}^{k} \theta_{j}\left(P_{j}\right)_{L, s}(z) y^{*}\left(y_{j}\right)\right| .
$$

Usaremos agora as seguintes ferramentas, na ordem indicada: (i) as igualdades em (4.14), (ii) a igualdade (4.13), (iii) o Teorema de Hahn-Banach na forma $\|x\|=\sup _{\|\varphi\| \leq 1}|\varphi(x)|$, (iv) a Desigualdade de Hölder para $\frac{1}{p}+\frac{1}{p^{*}}=1$.

$$
\begin{aligned}
\left(\sum_{j=m}^{k}\left|\left(P_{j}\right)_{L, s}(z) y^{*}\left(y_{j}\right)\right|^{p}\right)^{\frac{1}{p}} & =\left|\sum_{j=m}^{k} \theta_{j}\left(P_{j}\right)_{L, s}(z) y^{*}\left(y_{j}\right)\right|=\left|y^{*}\left(\sum_{j=m}^{k} \theta_{j}\left(P_{j}\right)_{L, s}(z) y_{j}\right)\right| \\
& \leq\left\|y^{*}\right\| \cdot\left\|\sum_{j=m}^{k} \theta_{j}\left(P_{j}\right)_{L, s}(z) y_{j}\right\| \leq\left\|\sum_{j=m}^{k} \theta_{j}\left(P_{j}\right)_{L, s}(z) y_{j}\right\|
\end{aligned}
$$




$$
\begin{aligned}
& =\left\|\left(\sum_{j=m}^{k}\left(P_{j}\right)_{L, s} \otimes\left(\theta_{j} y_{j}\right)\right)(z)\right\| \leq\left\|\sum_{j=m}^{k}\left(P_{j}\right)_{L, s} \otimes\left(\theta_{j} y_{j}\right)\right\| \cdot\|z\| \\
& \leq\left\|\sum_{j=m}^{k}\left(P_{j}\right)_{L, s} \otimes\left(\theta_{j} y_{j}\right)\right\|=\left\|\sum_{j=m}^{k} \theta_{j}\left(P_{j}\right)_{L, s} \otimes y_{j}\right\|=\left\|\sum_{j=m}^{k} \theta_{j} P_{j} \otimes y_{j}\right\| \\
& =\sup _{\|\widetilde{x}\| \leq 1}\left\|\left(\sum_{j=m}^{k} \theta_{j} P_{j} \otimes y_{j}\right)(\widetilde{x})\right\| \\
& =\sup _{\|\widetilde{x}\| \leq 1}\left\|\sum_{j=m}^{k} \theta_{j} P_{j}(\widetilde{x}) y_{j}\right\| \\
& =\sup _{\|\widetilde{x}\| \leq 1} \sup _{\left\|\widetilde{y}^{*}\right\| \leq 1}\left|\widetilde{y^{*}}\left(\sum_{j=m}^{k} \theta_{j} P_{j}(\widetilde{x}) y_{j}\right)\right| \\
& =\sup _{\|\widetilde{x}\| \leq 1,\left\|\widetilde{y}^{*}\right\| \leq 1}\left|\sum_{j=m}^{k} \theta_{j} P_{j}(\widetilde{x}) \widetilde{y^{*}}\left(y_{j}\right)\right| \\
& \leq \sup _{\|\widetilde{x}\| \leq 1,\left\|\widetilde{y^{*}}\right\| \leq 1} \sum_{j=m}^{k}\left|\theta_{j} P_{j}(\widetilde{x}) \widetilde{y^{*}}\left(y_{j}\right)\right| \\
& \leq \sup _{\|\widetilde{x}\| \leq 1,\left\|\widetilde{y}^{*}\right\| \leq 1}\left(\sum_{j=m}^{k}\left|\theta_{j}\right|^{p^{*}}\right) \cdot\left(\sum_{j=m}^{\frac{1}{p^{*}}}\left|P_{j}(\widetilde{x}) \widetilde{y^{*}}\left(y_{j}\right)\right|^{p}\right)^{\frac{1}{p}} \\
& =\sup _{\|\widetilde{x}\| \leq 1,\left\|\tilde{y}^{*}\right\| \leq 1}\left(\sum_{j=m}^{k}\left|P_{j}(\widetilde{x}) \widetilde{y^{*}}\left(y_{j}\right)\right|^{p}\right)^{\frac{1}{p}} \cdot \\
& \leq
\end{aligned}
$$

Assim, tomando o supremo sobre $\|z\|_{\pi_{s}} \leq 1$ e $\left\|y^{*}\right\| \leq 1$, temos

$$
\sup _{\|z\|\left\|_{s} \leq 1,\right\| y^{*} \| \leq 1}\left(\sum_{j=m}^{k}\left|\left(P_{j}\right)_{L, s}(z) y^{*}\left(y_{j}\right)\right|^{p}\right)^{\frac{1}{p}} \leq \sup _{\|x\| \leq 1,\left\|y^{*}\right\| \leq 1}\left(\sum_{j=m}^{k}\left|P_{j}(x) y^{*}\left(y_{j}\right)\right|^{p}\right)^{\frac{1}{p}}
$$

para todos $k \geq m$. Aplicando (4.15) e (4.10),

$$
\begin{aligned}
\sup _{\|z\|_{\pi_{s}} \leq 1,\left\|y^{*}\right\| \leq 1}\left(\sum_{j=1}^{\infty}\left|\left(P_{j}\right)_{L, s}(z) y^{*}\left(y_{j}\right)\right|^{p}\right)^{\frac{1}{p}} & =\sup _{\|z\|_{\pi_{s}} \leq 1,\left\|y^{*}\right\| \leq 1} \sup _{k}\left(\sum_{j=1}^{k}\left|\left(P_{j}\right)_{L, s}(z) y^{*}\left(y_{j}\right)\right|^{p}\right)^{\frac{1}{p}} \\
& =\sup _{k} \sup _{\|z\| \pi_{s} \leq 1,\left\|y^{*}\right\| \leq 1}\left(\sum_{j=1}^{k}\left|\left(P_{j}\right)_{L, s}(z) y^{*}\left(y_{j}\right)\right|^{p}\right)^{\frac{1}{p}} \\
& \leq \sup _{k} \sup _{\|x\| \leq 1,\left\|y^{*}\right\| \leq 1}\left(\sum_{j=1}^{k}\left|P_{j}(x) y^{*}\left(y_{j}\right)\right|^{p}\right)^{\frac{1}{p}}
\end{aligned}
$$




$$
\begin{aligned}
& =\sup _{\|x\| \leq 1,\left\|y^{*}\right\| \leq 1} \sup _{k}\left(\sum_{j=1}^{k}\left|P_{j}(x) y^{*}\left(y_{j}\right)\right|^{p}\right)^{\frac{1}{p}} \\
& =\sup _{\|x\| \leq 1,\left\|y^{*}\right\| \leq 1}\left(\sum_{j=1}^{\infty}\left|P_{j}(x) y^{*}\left(y_{j}\right)\right|^{p}\right)^{\frac{1}{p}}<\infty .
\end{aligned}
$$

E usando (4.15) e (4.11),

$$
\begin{aligned}
\sup _{\|z\|_{\pi_{s}} \leq 1,\left\|y^{*}\right\| \leq 1} & \left(\sum_{j=m}^{\infty}\left|\left(P_{j}\right)_{L, s}(z) y^{*}\left(y_{j}\right)\right|^{p}\right)^{\frac{1}{p}}=\sup _{\|z\|_{\pi_{s}} \leq 1,\left\|y^{*}\right\| \leq 1} \sup _{k \geq m}\left(\sum_{j=m}^{k}\left|\left(P_{j}\right)_{L, s}(z) y^{*}\left(y_{j}\right)\right|^{p}\right)^{\frac{1}{p}} \\
& =\sup _{k \geq m} \sup _{\|z\|_{\pi_{s}} \leq 1,\left\|y^{*}\right\| \leq 1}\left(\sum_{j=m}^{k}\left|\left(P_{j}\right)_{L, s}(z) y^{*}\left(y_{j}\right)\right|^{p}\right)^{\frac{1}{p}} \\
& \leq \sup _{k \geq m\|x\| \leq 1,\left\|y^{*}\right\| \leq 1} \sup _{j=m}\left(\sum_{j=m}^{k}\left|P_{j}(x) y^{*}\left(y_{j}\right)\right|^{p}\right)^{\frac{1}{p}} \\
& =\sup _{\|x\| \leq 1,\left\|y^{*}\right\| \leq 1} \sup _{k \geq m}\left(\sum_{j=m}^{k}\left|P_{j}(x) y^{*}\left(y_{j}\right)\right|^{p}\right)^{\frac{1}{p}} \\
& \leq \sup _{\|x\| \leq 1,\left\|y^{*}\right\| \leq 1}\left(\sum_{j=m}^{\infty}\left|P_{j}(x) y^{*}\left(y_{j}\right)\right|^{p}\right)_{\substack{\frac{1}{p} \\
m \rightarrow \infty}}^{\longrightarrow}
\end{aligned}
$$

Isso prova que $\sum_{j=1}^{\infty} \lambda_{j}\left(P_{j}\right)_{L, s} \otimes y_{j}$ é uma representação $\sigma(p)$-nuclear de $P_{L, s}$, isto é, $P_{L, s}$ é um operador linear $\sigma(p)$-nuclear, donde segue que $P \in \mathcal{N}_{\sigma(p)} \circ \mathcal{P}\left({ }^{n} E ; F\right)$. E, combinando com (4.12) e (4.16), segue também que

$$
\begin{aligned}
\|P\|_{\mathcal{N}_{\sigma(p)} \circ \mathcal{P}} & =\left\|P_{L, s}\right\|_{\mathcal{N}_{\sigma(p)}} \leq\left\|\left(\lambda_{j}\right)_{j=1}^{\infty}\right\|_{p^{*}} \cdot \sup _{\|z\|_{\pi_{s}} \leq 1, y^{*} \in B_{F^{*}}}\left(\sum_{j=1}^{\infty}\left|\left(P_{j}\right)_{L, s}(z) y^{*}\left(y_{j}\right)\right|^{p}\right)^{\frac{1}{p}} \\
& \leq\left\|\left(\lambda_{j}\right)_{j=1}^{\infty}\right\|_{p^{*}} \cdot \sup _{x \in B_{E}, y^{*} \in B_{F^{*}}}\left(\sum_{j=1}^{\infty}\left|P_{j}(x) y^{*}\left(y_{j}\right)\right|^{p}\right)^{\frac{1}{p}} \leq(1+\varepsilon)\|P\|_{\mathcal{P}_{\mathcal{H N} \sigma(p)}}
\end{aligned}
$$

onde a primeira igualdade segue da Proposição 1.6.16. Como isso vale para todo $\varepsilon>0$, fazendo $\varepsilon \longrightarrow 0^{+}$, concluímos que $\|P\|_{\mathcal{N}_{\sigma(p)} \circ \mathcal{L}} \leq\|P\|_{\mathcal{P}_{\mathcal{H N} \sigma(p)}}$.

Corolário 4.3.10. Seja $1 \leq p \leq \infty$. A classe $\mathcal{P}_{\mathcal{H N} \sigma(p)}$ dos polinômios homogêneos hiper-

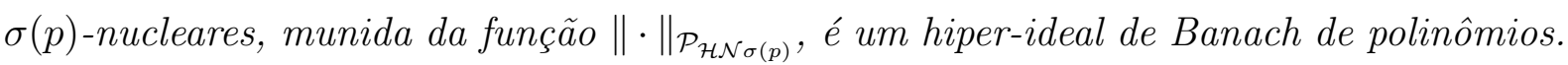


Demonstração. Basta combinar o Teorema 4.1.4 com a Proposição 4.3.9.

Concluímos este capítulo provando que funcionais lineares em espaços de polinômios homogêneos hiper- $\sigma(p)$-nucleares podem ser representados por operadores lineares quase$\tau(p)$-somantes.

Teorema 4.3.11. Seja $1 \leq p<\infty$ e suponha que o espaço $\mathcal{P}\left({ }^{n} E\right)$ possua a propriedade da aproximação limitada. Então, para todo espaço de Banach $F$, a hiper-transformada de Borel polinomial

$$
\mathfrak{B}_{n}:\left[\mathcal{P}_{\mathcal{H} \mathcal{N}_{\sigma(p)}}\left({ }^{n} E ; F\right)\right]^{*} \longrightarrow \mathcal{L}_{q \tau(p)}\left(\mathcal{P}\left({ }^{n} E\right) ; F^{*}\right), \mathfrak{B}_{n}(\phi)(P)(y)=\phi(P \otimes y),
$$

é um isomorfismo isométrico.

Demonstração. Pelo Exemplo 3.0.4 sabemos que a transformada de Borel linear

$$
\mathfrak{B}:\left[\mathcal{N}_{\sigma(p)}(E ; F)\right]^{*} \longrightarrow \mathcal{L}_{q \tau(p)}\left(E^{*} ; F^{*}\right), \mathfrak{B}(\phi)(\varphi)(y)=\phi(\varphi \otimes y),
$$

é um isomorfismo isométrico desde que $E^{*}$ tenha a propriedade da aproximação limitada. Como $\mathcal{L}_{q \tau(p)}$ é um semi-ideal à esquerda (Exemplo 3.1.6), o resultado segue combinando o Teorema 4.2.3 com a Proposição 4.3.9. 


\section{Capítulo 5}

\section{Hiper-ideais gerados por classes de sequências}

Hiper-ideais de operadores multilineares e de polinômios homogêneos desempenharam um papel central nesta tese até aqui. Além dos exemplos vistos até agora, muitos outros exemplos e também técnicas para gerar hiper-ideais podem ser encontrados em [20, 22]. Desenvolveremos neste capítulo final da tese uma nova técnica para gerar hiper-ideais de operadores multilineares e de polinômios homogêneos, e também de ideais bilaterais de polinômios homogêneos. Essa técnica é baseada no conceito de classes de sequências introduzido em [11].

\subsection{Hiper-ideais de operadores multilineares gerados por classes de sequências}

Uma primeira tentativa de tratar ideais de operadores multilineares por meio de classes de sequências foi feito em [69]. Os conceitos abaixo foram introduzidas em [11] e têm se mostrado frutíferos: aplicações e novos desenvolvimentos podem ser encontrados em $[1,10,12,13,14,30,64,65]$.

Fixemos algumas notações que simplificarão a exposição. Por $\left(e_{j}\right)_{j=1}^{\infty}$ denotamos os vetores canônicos dos espaços de sequências escalares, isto é, $e_{1}=(1,0,0,0, \ldots), e_{2}=$ $(0,1,0,0,0, \ldots), \ldots$ O símbolo $E \hookrightarrow F$ significa que o espaço de Banach $E$ é um subespaço vetorial do espaço de Banach $F$ e que $\|x\|_{F} \leq\|x\|_{E}$ para todo $x \in E$. Dada uma sequência 
$\left(x_{j}\right)_{j=1}^{\infty}$ em um espaço vetorial e dado $k \in \mathbb{N}$, definimos

$$
\left(x_{j}\right)_{j=1}^{k}:=\left(x_{1}, x_{2}, \ldots, x_{k}, 0,0,0, \ldots\right)
$$

Definição 5.1.1. Uma classe de sequências vetoriais, ou simplesmente uma classe de sequências, é uma regra $E \mapsto X(E)$ que associa a cada espaço de Banach $E$ um espaço de Banach $X(E)$ formado por sequências em $E$, isto é, um subespaço vetorial de $E^{\mathbb{N}}$ com as operações usuais, satisfazendo as seguintes condições:

(a) $c_{00}(E) \subseteq X(E)$;

(b) $X(E) \hookrightarrow \ell_{\infty}(E)$;

(c) $\left\|e_{j}\right\|_{X(\mathbb{K})}=1$, para todo $j \in \mathbb{N}$.

Condição de Compatibilidade: Dadas classes de sequências $X_{1}, \ldots, X_{n}$ e $Y$, dizemos que $X_{1}(\mathbb{K}) \cdots X_{n}(\mathbb{K}) \hookrightarrow Y(\mathbb{K})$ se $\left(\lambda_{j}^{1} \cdots \lambda_{j}^{n}\right)_{j=1}^{\infty} \in Y(\mathbb{K})$ e

$$
\left\|\left(\lambda_{j}^{1} \cdots \lambda_{j}^{n}\right)_{j=1}^{\infty}\right\|_{Y(\mathbb{K})} \leq \prod_{m=1}^{n}\left\|\left(\lambda_{j}^{m}\right)_{j=1}^{\infty}\right\|_{X_{m}(\mathbb{K})}
$$

sempre que $\left(\lambda_{j}^{m}\right)_{j=1}^{\infty} \in X_{m}(\mathbb{K}), m=1, \ldots, n$.

No caso em que $X_{1}=\cdots=X_{n}=X$, escrevemos simplesmente $X(\mathbb{K})^{n} \hookrightarrow Y(\mathbb{K})$.

Definição 5.1.2. Uma classe de sequências $X$ é dita:

- Linearmente estável se para todos espaços de Banach $E$ e $F$ e todo operador $u \in$ $\mathcal{L}(E ; F)$, tem-se $\left(u\left(x_{j}\right)\right)_{j=1}^{\infty} \in X(F)$ sempre que $\left(x_{j}\right)_{j=1}^{\infty} \in X(E)$ e, neste caso, o operador linear induzido

$$
\widetilde{u}: X(E) \longrightarrow X(F), \widetilde{u}\left(\left(x_{j}\right)_{j=1}^{\infty}\right)=\left(u\left(x_{j}\right)\right)_{j=1}^{\infty},
$$

é contínuo e $\|\widetilde{u}\|=\|u\|$.

- Multilinearmente estável se para todos $n \in \mathbb{N}$, espaços de Banach $E_{1}, \ldots, E_{n}$ e $F$ e $A \in$ $\mathcal{L}\left(E_{1}, \ldots, E_{n} ; F\right),\left(A\left(x_{j}^{1}, \ldots, x_{j}^{n}\right)\right)_{j=1}^{\infty} \in X(F)$ sempre que $\left(x_{j}^{m}\right)_{j=1}^{\infty} \in X\left(E_{m}\right), m=1, \ldots, n$ e, neste caso, o operador $n$-linear induzido

$$
\widetilde{A}: X\left(E_{1}\right) \times \cdots \times X\left(E_{n}\right) \longrightarrow X(F), \widetilde{A}\left(\left(x_{j}^{1}\right)_{j=1}^{\infty}, \ldots,\left(x_{j}^{n}\right)_{j=1}^{\infty}\right)=\left(A\left(x_{j}^{1}, \ldots, x_{j}^{n}\right)\right)_{j=1}^{\infty},
$$

é contínuo e $\|\widetilde{A}\|=\|A\|$. 
É claro que toda classe multilinearmente estável é linearmente estável.

Exemplo 5.1.3. Seja $\left(r_{j}\right)_{j=1}^{\infty}$ a sequência das funções de Rademacher em [0,1]. Uma sequência $\left(x_{j}\right)_{j=1}^{\infty}$ em $E$ é quase incondicionalmente somável se a série $\sum_{j} r_{j}(t) x_{j}$ converge em $L_{p}([0,1] ; E)$ para algum (ou, equivalentemente, para todo) $0<p<\infty$. O conjunto dessas sequências, denotado por $\operatorname{Rad}(E)$, é um espaço de Banach munido da norma

$$
\left\|\left(x_{j}\right)_{j=1}^{\infty}\right\|_{\operatorname{Rad}(E)}=\left\|\sum_{j} r_{j} x_{j}\right\|_{L_{2}(E)}=\left(\int_{0}^{1}\left\|\sum_{j=1}^{\infty} r_{j}(t) x_{j}\right\|^{2} d t\right)^{\frac{1}{2}} .
$$

Definimos ainda o espaço de Banach

$$
\operatorname{RAD}(E):=\left\{\left(x_{j}\right)_{j=1}^{\infty} \in E^{\mathbb{N}}:\left\|\left(x_{j}\right)_{j=1}^{\infty}\right\|_{\operatorname{RAD}(E)}=\sup _{k}\left\|\left(x_{j}\right)_{j=1}^{k}\right\|_{\operatorname{Rad}(E)}<\infty\right\} .
$$

Para maiores informações sobre esses espaços veja [34, 73].

Consideramos também o subespaço fechado de $\ell_{p}^{w}(E), 1 \leq p<\infty$, formado pelas sequências incondicionalmente $p$-somáveis:

$$
\ell_{p}^{u}(E)=\left\{\left(x_{j}\right)_{j=1}^{\infty} \in \ell_{p}^{w}(E): \lim _{k}\left\|\left(x_{j}\right)_{j=k}^{\infty}\right\|_{w, p}=0\right\} .
$$

Em [33] esses espaços são denotados por $\ell_{p}^{w, 0}(E)$.

Por $c_{0}^{w}(E)$ denotamos o subespaço fechado de $c_{0}(E)$ formado pelas sequências fracamente nulas. Por fim, $\ell_{p}\langle E\rangle$ é o espaço de Banach das sequências em $E$ que são Cohen fortemente $p$-somáveis (veja [11]).

As seguintes classes de sequências são multilinearmente estáveis:

$$
\begin{gathered}
E \mapsto \ell_{\infty}(E), E \mapsto c_{0}(E), E \mapsto \operatorname{Rad}(E), E \mapsto \operatorname{RAD}(E), E \mapsto \ell_{1}^{w}(E), E \mapsto \ell_{1}^{u}(E), \\
E \mapsto \ell_{p}(E), E \mapsto \ell_{p}\langle E\rangle, 1 \leq p<+\infty .
\end{gathered}
$$

As seguintes classes de sequências são linearmente estáveis mas não são multilinearmente estáveis:

$$
E \mapsto c_{0}^{w}(E), E \mapsto \ell_{p}^{w}(E), E \mapsto \ell_{p}^{u}(E), 1<p<+\infty
$$

Tudo isso pode ser encontrado em [11].

Vejamos como classes de sequências geram multi-ideais. 
Definição 5.1.4. Para $n \in \mathbb{N}$, classes de sequências $X_{1}, \ldots, X_{n}, Y$ e espaços de Banach $E_{1}, \ldots, E_{n}, F$, diz-se que um operador $A \in \mathcal{L}\left(E_{1}, \ldots, E_{n} ; F\right)$ é $\left(X_{1}, \ldots, X_{n} ; Y\right)$-somante se $\left(A\left(x_{j}^{1}, \ldots, x_{j}^{n}\right)\right)_{j=1}^{\infty} \in Y(F)$ sempre que $\left(x_{j}^{m}\right)_{j=1}^{\infty} \in X_{m}\left(E_{m}\right), m=1, \ldots, n$.

Neste caso escrevemos $A \in \mathcal{L}_{X_{1}, \ldots, X_{n} ; Y}\left(E_{1}, \ldots, E_{n} ; F\right)$ e, de acordo com [11, Proposition 2.4], o operador $n$-linear induzido

$$
\widetilde{A}: X_{1}\left(E_{1}\right) \times \cdots \times X_{n}\left(E_{n}\right) \longrightarrow Y(F), \widetilde{A}\left(\left(x_{j}^{1}\right)_{j=1}^{\infty}, \ldots,\left(x_{j}^{n}\right)_{j=1}^{\infty}\right)=\left(A\left(x_{j}^{1}, \ldots, x_{j}^{n}\right)\right)_{j=1}^{\infty}
$$

é contínuo. Define-se $\|A\|_{X_{1}, \ldots, X_{n} ; Y}=\|\widetilde{A}\|$.

No caso em que $X_{1}=\cdots=X_{n}=X$, dizemos que $A$ é $(X ; Y)$-somante e escrevemos simplesmente $\mathcal{L}_{X ; Y}\left(E_{1}, \ldots, E_{n} ; F\right)$ e $\|\cdot\|_{X ; Y}$.

Teorema 5.1.5. [11, Theorem 3.6] Sejam $n \in \mathbb{N}$ e $X_{1}, \ldots, X_{n}, Y$ classes de sequências linearmente estáveis satisfazendo a condição de compatibilidade. Então $\left(\mathcal{L}_{X_{1}, \ldots, X_{n} ; Y}, \| \cdot\right.$ $\left.\|_{X_{1}, \ldots, X_{n} ; Y}\right)$ é um ideal de Banach de operadores n-lineares.

A seguinte consequência é imediata:

Corolário 5.1.6. Sejam $X$ e $Y$ classes de sequências linearmente estáveis tais que $X(\mathbb{K})^{n} \hookrightarrow$ $Y(\mathbb{K})$ para todo $n \in \mathbb{N}$. Então $\left(\mathcal{L}_{X ; Y},\|\cdot\|_{X ; Y}\right)$ é um multi-ideal de Banach.

Com uma condição a mais sobre $X$ obtemos hiper-ideais:

Teorema 5.1.7. Sejam $X$ e $Y$ classes de sequências com $X$ multilinearmente estável, $Y$ linearmente estável e $X(\mathbb{K})^{n} \hookrightarrow Y(\mathbb{K})$ para todo $n \in \mathbb{N}$. Então $\left(\mathcal{L}_{X ; Y},\|\cdot\|_{X ; Y}\right)$ é um hiper-ideal de Banach de operadores multilineares.

Demonstração. Já sabemos do Corolário 5.1.6 que $\mathcal{L}_{X ; Y}$ é um multi-ideal de Banach. Resta provar a propriedade de hiper-ideal. Para isso sejam $B_{1} \in \mathcal{L}\left(G_{1}, \ldots, G_{m_{1}} ; E_{1}\right), \ldots, B_{n} \in$ $\mathcal{L}\left(G_{m_{n-1}+1}, \ldots, G_{m_{n}} ; E_{n}\right), t \in \mathcal{L}(F ; H)$ e $A \in \mathcal{L}_{X ; Y}\left(E_{1}, \ldots, E_{n} ; F\right)$. Dadas sequências $\left(x_{j}^{k}\right)_{j=1}^{\infty} \in X\left(G_{k}\right), k=1, \ldots, m_{n}$, como $X$ é multilinearmente estável temos

$$
\left(B_{1}\left(x_{j}^{1}, \ldots, x_{j}^{m_{1}}\right)\right)_{j=1}^{\infty} \in X\left(E_{1}\right), \ldots,\left(B_{n}\left(x_{j}^{m_{n-1}+1}, \ldots, x_{j}^{m_{n}}\right)\right)_{j=1}^{\infty} \in X\left(E_{n}\right)
$$

e

$$
\begin{gathered}
\left\|\widetilde{B_{1}}: X\left(G_{1}\right) \times \cdots \times X\left(G_{m_{1}}\right) \longrightarrow E_{1}\right\|=\left\|B_{1}\right\|, \ldots, \\
\left\|\widetilde{B_{n}}: X\left(G_{m_{n-1}+1}\right) \times \cdots \times X\left(G_{m_{n}}\right) \longrightarrow E_{n}\right\|=\left\|B_{n}\right\| .
\end{gathered}
$$


Usando que $A$ é $(X ; Y)$-somante, segue que

$$
\left(A\left(B_{1}\left(x_{j}^{1}, \ldots, x_{j}^{m_{1}}\right), \ldots, B_{n}\left(x_{j}^{m_{n-1}+1}, \ldots, x_{j}^{m_{n}}\right)\right)\right)_{j=1}^{\infty} \in Y(F)
$$

$\mathrm{e}$

$$
\left\|\widetilde{A}: X\left(E_{1}\right) \times \cdots \times X\left(E_{n}\right) \longrightarrow Y(F)\right\|=\|A\|_{X ; Y} .
$$

E como $Y$ é linearmente estável, temos $\|\widetilde{t}: Y(F) \longrightarrow Y(H)\|=\|t\| \mathrm{e}$

$$
\begin{gathered}
\left(\left(t \circ A \circ\left(B_{1}, \ldots, B_{n}\right)\right)\left(x_{j}^{1}, \ldots, x_{j}^{m_{n}}\right)\right)_{j=1}^{\infty}= \\
=\left(t\left(A\left(B_{1}\left(x_{j}^{1}, \ldots, x_{j}^{m_{1}}\right), \ldots, B_{n}\left(x_{j}^{m_{n-1}+1}, \ldots, x_{j}^{m_{n}}\right)\right)\right)\right)_{j=1}^{\infty} \in Y(H) .
\end{gathered}
$$

Isso prova que $\left(t \circ A \circ\left(B_{1}, \ldots, B_{n}\right)\right) \in \mathcal{L}_{X ; Y}\left(G_{1}, \ldots, G_{m_{n}} ; H\right)$. E da igualdade acima também segue que

$$
\left[t \circ A \circ\left(B_{1}, \ldots, B_{n}\right)\right]^{\sim}=\widetilde{t} \circ \widetilde{A} \circ\left(\widetilde{B_{1}}, \ldots, \widetilde{B_{n}}\right)
$$

Daí,

$$
\begin{aligned}
\left\|t \circ A \circ\left(B_{1}, \ldots, B_{n}\right)\right\|_{X ; Y} & =\left\|\left[t \circ A \circ\left(B_{1}, \ldots, B_{n}\right)\right]^{\sim}\right\| \\
& =\left\|\widetilde{t} \circ \widetilde{A} \circ\left(\widetilde{B_{1}}, \ldots, \widetilde{B_{n}}\right)\right\| \\
& \leq\|\widetilde{t}\| \cdot\|\widetilde{A}\| \cdot\left\|\widetilde{B_{1}}\right\| \cdots\left\|\widetilde{B_{n}}\right\| \\
& =\|t\| \cdot\|A\|_{X ; Y} \cdot\left\|B_{1}\right\| \cdots\left\|B_{n}\right\|,
\end{aligned}
$$

o que completa a demonstração.

Antes de dar exemplos concretos em que $\mathcal{L}_{X ; Y}$ é um hiper-ideal, vejamos uma propriedade importante dessa classe que, aparentemente, passou despercebida até agora e que nos será útil na próxima seção.

Definição 5.1.8. Para $n \in \mathbb{N}$ denotamos por $S_{n}$ o conjunto das permutações do conjunto $\{1,2, \ldots, n\}$. Dados um operador $A \in \mathcal{L}\left({ }^{n} E ; F\right)$ e uma permutação $\sigma \in S_{n}$, definimos $A_{\sigma} \in \mathcal{L}\left({ }^{n} E ; F\right)$ por

$$
A_{\sigma}\left(x_{1}, \ldots, x_{n}\right)=A\left(x_{\sigma(1)}, \ldots, x_{\sigma(n)}\right) .
$$

Definimos agora a simetrização $A_{s} \in \mathcal{L}\left({ }^{n} E ; F\right)$ de $A$ por

$$
A_{s}\left(x_{1}, \ldots, x_{n}\right)=\frac{1}{n !} \cdot \sum_{\sigma \in S_{n}} A_{\sigma}\left(x_{1}, \ldots, x_{n}\right) .
$$


Por uma subclasse normada $\left(\mathcal{G},\|\cdot\|_{\mathcal{G}}\right)$ da classe dos operadores multilineares contínuos entre espaços de Banach entendemos uma correspondência que para cada $n \in \mathbb{N}$ e espaços de Banach $E_{1}, \ldots, E_{n}, F$, associa um subespaço vetorial $\mathcal{G}\left(E_{1}, \ldots, E_{n} ; F\right)$ de $\mathcal{L}\left(E_{1}, \ldots, E_{n} ; F\right)$ munido de uma norma $\|\cdot\|_{\mathcal{G}}$. O conceito a seguir é devido a Floret e García [43].

Definição 5.1.9. Dizemos que uma subclasse normada $\left(\mathcal{G},\|\cdot\|_{\mathcal{G}}\right)$ da classe dos operadores multilineares contínuos entre espaços de Banach é fortemente simétrica se, para quaisquer espaços de Banach $E$ e $F, n \in \mathbb{N}, \sigma \in S_{n}$ e $A \in \mathcal{G}\left({ }^{n} E ; F\right)$, tem-se $A_{\sigma} \in \mathcal{G}\left({ }^{n} E ; F\right)$ e $\left\|A_{\sigma}\right\|_{\mathcal{G}}=\|A\|_{\mathcal{G}}$.

É claro que, neste caso, $A_{s} \in \mathcal{G}\left({ }^{n} E ; F\right)$ sempre que $A \in \mathcal{G}\left({ }^{n} E ; F\right)$.

No Teorema 5.1.5, o fato das classes de sequências serem linearmente estáveis é usado para provar a propriedade de ideal, e a condição de compatibilidade é usada para provar a continência dos operadores de tipo finito. Então, para todas classes de sequências $X$ e $Y,\left(\mathcal{L}_{X ; Y},\|\cdot\|_{X ; Y}\right)$ é uma classe normada de operadores multilineares.

Proposição 5.1.10. Para todas classes de sequências $X$ e $Y,\left(\mathcal{L}_{X ; Y}\left({ }^{n} E ; F\right),\|\cdot\|_{X ; Y}\right)$ é uma classe fortemente simétrica.

Demonstração. Provemos primeiramente que, para todos $n \in \mathbb{N}$, espaços de Banach $G$ e $H, \sigma \in S_{n}$ e $T \in \mathcal{L}\left({ }^{n} G ; H\right)$, é verdade que $\left\|T_{\sigma}\right\|=\|T\|$. Da igualdade de conjuntos

$$
\left\{T\left(z_{\sigma(1)}, \ldots, z_{\sigma(n)}\right): z_{1}, \ldots, z_{n} \in B_{G}\right\}=\left\{T\left(z_{1}, \ldots, z_{n}\right): z_{1}, \ldots, z_{n} \in B_{G}\right\}
$$

segue a igualdade

$$
\left\{\left\|T\left(z_{\sigma(1)}, \ldots, z_{\sigma(n)}\right)\right\|: z_{1}, \ldots, z_{n} \in B_{G}\right\}=\left\{\left\|T\left(z_{1}, \ldots, z_{n}\right)\right\|: z_{1}, \ldots, z_{n} \in B_{G}\right\}
$$

e portanto

$$
\begin{aligned}
\left\|T_{\sigma}\right\| & =\sup \left\{\left\|T_{\sigma}\left(z_{1}, \ldots, z_{n}\right)\right\|: z_{1}, \ldots, z_{n} \in B_{G}\right\} \\
& =\sup \left\{\left\|T\left(z_{\sigma(1)}, \ldots, z_{\sigma(n)}\right)\right\|: z_{1}, \ldots, z_{n} \in B_{G}\right\} \\
& =\sup \left\{\left\|T\left(z_{1}, \ldots, z_{n}\right)\right\|: z_{1}, \ldots, z_{n} \in B_{G}\right\}=\|T\| .
\end{aligned}
$$

Sejam agora $n \in \mathbb{N}, E$ e $F$ espaços de Banach quaisquer e $\sigma \in S_{n}$. Tomando $A \in \mathcal{L}_{X ; Y}\left({ }^{n} E ; F\right)$ devemos mostrar que $A_{\sigma} \in \mathcal{L}_{X ; Y}\left({ }^{n} E ; F\right)$ e $\left\|A_{\sigma}\right\|_{X ; Y}=\|A\|_{X ; Y}$. Sabemos que o operador induzido $\widetilde{A}: X(E)^{n} \longrightarrow Y(F)$ é bem definido, $n$-linear e contínuo. 
Podemos então considerar a permutação de $\widetilde{A}$ por $\sigma,(\widetilde{A})_{\sigma}: X(E)^{n} \longrightarrow Y(F)$, o qual é igualmente $n$-linear e contínuo. E podemos considerar ainda o operador induzido por $A_{\sigma}$, $\widetilde{A_{\sigma}}: X(E)^{n} \longrightarrow F^{\mathbb{N}}$. Vejamos que

$$
(\widetilde{A})_{\sigma}=\widetilde{A_{\sigma}}
$$

Com efeito, para toda sequência $\left(x_{j}^{m}\right)_{j=1}^{\infty} \in X(E), m=1, \ldots, n$,

$$
\begin{aligned}
(\widetilde{A})_{\sigma}\left(\left(x_{j}^{1}\right)_{j=1}^{\infty}, \ldots,\left(x_{j}^{n}\right)_{j=1}^{\infty}\right) & =\widetilde{A}\left(\left(x_{j}^{\sigma(1)}\right)_{j=1}^{\infty}, \ldots,\left(x_{j}^{\sigma(n)}\right)_{j=1}^{\infty}\right) \\
& =\left(A\left(x_{j}^{\sigma(1)}, \ldots, x_{j}^{\sigma(n)}\right)\right)_{j=1}^{\infty} \\
& =\left(A_{\sigma}\left(x_{j}^{1}, \ldots, x_{j}^{n}\right)\right)_{j=1}^{\infty} \\
& =\widetilde{A_{\sigma}}\left(\left(x_{j}^{1}\right)_{j=1}^{\infty}, \ldots,\left(x_{j}^{n}\right)_{j=1}^{\infty}\right) .
\end{aligned}
$$

Como $(\widetilde{A})_{\sigma}$ toma valores em $Y(F)$, segue de $(5.2)$ que $\widetilde{A_{\sigma}}$ também toma valores em $Y(F)$, e isso quer dizer que $A_{\sigma} \in \mathcal{L}_{X ; Y}\left({ }^{n} E ; F\right)$. Mais ainda,

$$
\|A\|_{X ; Y}=\|\widetilde{A}\|_{\mathcal{L}\left({ }^{n} X(E) ; Y(F)\right)}=\left\|(\widetilde{A})_{\sigma}\right\|_{\mathcal{L}\left({ }^{n} X(E) ; Y(F)\right)}=\left\|\widetilde{A_{\sigma}}\right\|_{\mathcal{L}\left({ }^{n} X(E) ; Y(F)\right)}=\left\|A_{\sigma}\right\|_{X ; Y}
$$

onde a segunda igualdade segue de (5.1) e a terceira de (5.2).

A seguir mostraremos, com exemplos, como o Teorema 5.1.7 permite concluir que vários multi-ideais já estudados são na verdade hiper-ideais de Banach de operadores multilineares.

Exemplo 5.1.11. Operadores multilineares absolutamente somantes foram introduzidos em [5] e posteriormente estudados em um grande número de trabalhos (veja, por exemplo, as referências em [14]). Essa classe é um caso particular das classes tratadas no Exemplo 1.4.5. Por definição, um operador $A \in \mathcal{L}\left(E_{1}, \ldots, E_{n} ; F\right)$ é absolutamente somante se $\left(A\left(x_{j}^{1}, \ldots, x_{j}^{n}\right)\right)_{j=1}^{\infty} \in \ell_{1}(F)$ sempre que $\left(x_{j}^{m}\right)_{j=1}^{\infty} \in \ell_{1}^{w}\left(E_{m}\right), m=1, \ldots, n$. Na nossa notação, trata-se da classe $\mathcal{L}_{\ell_{1}^{w}(\cdot) \ell_{1}(\cdot)}$. Vimos no Exemplo 5.1 .3 que as classes de sequências $\ell_{1}(\cdot)$ e $\ell_{1}^{w}(\cdot)$ são multilinearmente estáveis. Segue então do Teorema 5.1.7 que o multi-ideal dos operadores absolutamente somantes é um hiper-ideal de Banach.

Exemplo 5.1.12. Operadores multilineares fracamente somantes foram estudados em $[8,11,49,62,70]$. Uma caracterização conhecida é que um operador $A \in \mathcal{L}\left(E_{1}, \ldots, E_{n} ; F\right)$ é fracamente somante se, e somente se, $\left(A\left(x_{j}^{1}, \ldots, x_{j}^{n}\right)\right)_{j=1}^{\infty} \in \ell_{1}^{w}(F)$ sempre que $\left(x_{j}^{m}\right)_{j=1}^{\infty} \in$

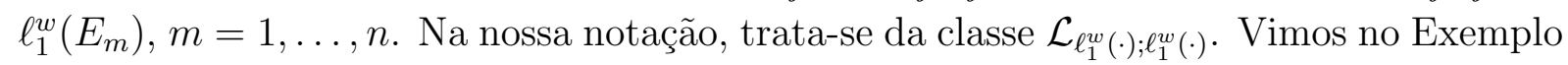
5.1.3 que a classe de sequências $\ell_{1}^{w}(\cdot)$ é multilinearmente estável. Segue então do Teorema 5.1.7 que o multi-ideal dos operadores fracamente somantes é um hiper-ideal de Banach. 
Exemplo 5.1.13. Operadores multilineares de tipo $p, 1 \leq p \leq 2$, foram introduzidos em [10] (veja também [14]). Conforme provado em [10], um operador $A \in \mathcal{L}\left(E_{1}, \ldots, E_{n} ; F\right)$ é de tipo $p$ se, e somente se, $\left(A\left(x_{j}^{1}, \ldots, x_{j}^{n}\right)\right)_{j=1}^{\infty} \in \operatorname{Rad}(F)$ sempre que $\left(x_{j}^{m}\right)_{j=1}^{\infty} \in \ell_{p}\left(E_{m}\right)$, $m=1, \ldots, n$. Na nossa notação, trata-se da classe $\mathcal{L}_{\ell_{p}(\cdot) ; \operatorname{Rad}(\cdot)}$. Vimos no Exemplo 5.1.3 que as classes de sequências $\ell_{p}(\cdot)$ e $\operatorname{Rad}(\cdot)$ são multilinearmente estáveis. Segue então do Teorema 5.1.7 que o multi-ideal dos operadores de tipo $p$ é um hiper-ideal de Banach.

Exemplo 5.1.14. Operadores multilineares de cotipo $q, 2 \leq q<+\infty$, foram introduzidos em [10] (veja também [14]). Conforme provado em [10], um operador $A \in$ $\mathcal{L}\left(E_{1}, \ldots, E_{n} ; F\right)$ é de cotipo $q$ se, e somente se, $\left(A\left(x_{j}^{1}, \ldots, x_{j}^{n}\right)\right)_{j=1}^{\infty} \in \ell_{q}(F)$ sempre que $\left(x_{j}^{m}\right)_{j=1}^{\infty} \in \operatorname{Rad}\left(E_{m}\right), m=1, \ldots, n$. Na nossa notação, trata-se da classe $\mathcal{L}_{\operatorname{Rad}(\cdot) ; \ell_{q}(\cdot) \text {. Vi- }}$ mos no Exemplo 5.1.3 que as classes de sequências $\ell_{q}(\cdot)$ e $\operatorname{Rad}(\cdot)$ são multilinearmente estáveis. Segue então do Teorema 5.1.7 que o multi-ideal dos operadores de cotipo $q$ é um hiper-ideal de Banach.

Exemplo 5.1.15. Operadores multilineares Cohen quase somantes apareceram em [27]. Um operador $A \in \mathcal{L}\left(E_{1}, \ldots, E_{n} ; F\right)$ é Cohen quase somante se, e somente se,

$$
\left(A\left(x_{j}^{1}, \ldots, x_{j}^{n}\right)\right)_{j=1}^{\infty} \in \ell_{2}\langle F\rangle \text { sempre que }\left(x_{j}^{m}\right)_{j=1}^{\infty} \in \operatorname{Rad}(F), m=1, \ldots, n \text {. }
$$

Na nossa notação, trata-se da classe $\mathcal{L}_{\operatorname{Rad}(\cdot) ; \ell_{2}\langle\cdot\rangle}$. Vimos no Exemplo 5.1 .3 que as classes de sequências $\operatorname{Rad}(\cdot)$ e $\ell_{2}\langle\cdot\rangle$ são multilinearmente estáveis. Segue então do Teorema 5.1.7 que o multi-ideal dos operadores Cohen quase somantes é um hiper-ideal de Banach.

Além de classes já estudadas, nossa abordagem permite, combinando as classes de sequências do Exemplo 5.1.3 com o Teorema 5.1.7, exibir uma enorme quantidade de hiper-ideais de operadores multilineares que ainda não foram estudados. Apresentamos apenas um exemplo a título de ilustração.

Exemplo 5.1.16. Para $1 \leq p<\infty$, como as classes $\ell_{p}(\cdot)$ e $\ell_{p}\langle\cdot\rangle$ são multilinearmente estáveis, segue do Teorema 5.1 .7 que o multi-ideal $\mathcal{L}_{\ell_{p}(\cdot) ; \ell_{p}\langle\cdot\rangle}$ é um hiper-ideal de Banach. No caso linear essa classe coincide com o ideal dos operadores Cohen fortemente $p$-somantes, mas no caso multilinear essa classe não coincide com a classe dos operadores multilineares Cohen fortemente $p$-somantes estudados em [2, 27, 28, 29, 54, 55, 63]. De fato, de acordo com [29], um operador $n$-linear é Cohen fortemente $p$-somante se transforma sequências de $\ell_{n p}(\cdot)$ em sequências de $\ell_{p}\langle\cdot\rangle$, e não sequências $\ell_{p}(\cdot)$ em sequências de $\ell_{p}\langle\cdot\rangle$. 


\subsection{Classes de sequências e ideais de polinômios}

Dadas classes de sequências $X$ e $Y$ satisfazendo a condição de compatibilidade com $X$ multilinearmente estável e $Y$ linearmente estável, sabemos da seção anterior que a classe $\mathcal{L}_{X ; Y}$ dos operadores multilineares $(X ; Y)$-somantes é um hiper-ideal de Banach. Nosso objetivo nesta seção é estudar o caso polinomial. Diante disso, temos três possibilidades para definir polinômios homogêneos $(X ; Y)$-somantes: podemos considerar os polinômios $P \in \mathcal{P}\left({ }^{n} E ; F\right)$ tais que:

- $\left(P\left(x_{j}\right)\right)_{j=1}^{\infty} \in Y(F)$, sempre que $\left(x_{j}\right)_{j=1}^{\infty} \in X(E), \mathrm{OU}$

- $\check{P} \in \mathcal{L}_{X ; Y}\left({ }^{n} E ; F\right), \mathrm{OU}$

- Existe $A \in \mathcal{L}_{X ; Y}\left({ }^{n} E ; F\right)$ tal que $\widehat{A}=P$.

A primeira opção foi tratada em [1], mas foi ali desenvolvida apenas para algumas classes de sequências específicas. Veremos a seguir que essas três maneiras são equivalentes, o que não deixa dúvidas sobre a maneira adequada de se definir polinômios $(X ; Y)$-somantes. Além disso, cada uma dessas três opções traz consigo uma maneira natural de definir uma norma no espaço dos polinômios $(X ; Y)$-somantes. Também veremos as relações entre essas normas.

Algumas partes do teorema a seguir encontram-se em [1, Proposition 2.6].

Teorema 5.2.1. Sejam $X$ e $Y$ classes de sequências. As seguintes afirmações são equivalentes para todo polinômio $P \in \mathcal{P}\left({ }^{n} E ; F\right)$ :

(a) $\check{P} \in \mathcal{L}_{X ; Y}\left({ }^{n} E ; F\right)$.

(b) Existe $A \in \mathcal{L}_{X ; Y}\left({ }^{n} E ; F\right)$ tal que $\widehat{A}=P$.

(c) $\left(P\left(x_{j}\right)\right)_{j=1}^{\infty} \in Y(F)$ sempre que $\left(x_{j}\right)_{j=1}^{\infty} \in X(E)$.

(d) O operador induzido

$$
\widetilde{P}: X(E) \longrightarrow Y(F), \widetilde{P}\left(\left(x_{j}\right)_{j=1}^{\infty}\right)=\left(P\left(x_{j}\right)\right)_{j=1}^{\infty},
$$

está bem definido e é um polinômio n-homogêneo contínuo.

Nas condições acima,

$$
\|\widetilde{P}\| \leq\|\check{P}\|_{X ; Y}=\inf \left\{\|A\|_{X ; Y}: A \in \mathcal{L}_{X ; Y} \text { e } \widehat{A}=P\right\} \leq \frac{n^{n}}{n !}\|\widetilde{P}\| .
$$


Demonstração. A implicação $(a) \Longrightarrow(b)$ é imediata pois $\widehat{\widetilde{P}}=P$.

$(b) \Longrightarrow(c)$ Por hipótese existe $A \in \mathcal{L}_{X ; Y}\left({ }^{n} E ; F\right)$ tal que $\widehat{A}=P$. Pela definição de $\mathcal{L}_{X ; Y}$ sabemos que $\left(A\left(x_{j}, \ldots, x_{j}\right)\right)_{j=1}^{\infty} \in Y(F)$ sempre que $\left(x_{j}\right)_{j=1}^{\infty} \in X(E)$. Logo

$$
\left(P\left(x_{j}\right)\right)_{j=1}^{\infty}=\left(\widehat{A}\left(x_{j}, \ldots, x_{j}\right)\right)_{j=1}^{\infty}=\left(A\left(x_{j}, \ldots, x_{j}\right)\right)_{j=1}^{\infty} \in Y(F)
$$

sempre que $\left(x_{j}\right)_{j=1}^{\infty} \in X(E)$.

$(c) \Longrightarrow(a)$ Essa implicação está implícita na demonstração de [1, Proposition 2.6], mas por completude exibiremos os detalhes. Sejam $\left(x_{j}^{1}\right)_{j=1}^{\infty}, \ldots,\left(x_{j}^{n}\right)_{j=1}^{\infty} \in X(E)$. Como $X(E)$ é espaço vetorial, para cada escolha de sinais $\varepsilon_{1}, \ldots, \varepsilon_{n}= \pm 1$, temos

$$
\left(\varepsilon_{1} x_{j}^{1}+\cdots+\varepsilon_{n} x_{j}^{n}\right)_{j=1}^{\infty}=\varepsilon_{1}\left(x_{j}^{1}\right)_{j=1}^{\infty}+\cdots+\varepsilon_{n}\left(x_{j}^{n}\right)_{j=1}^{\infty} \in X(E) .
$$

Por hipótese segue que $\left(P\left(\varepsilon_{1} x_{j}^{1}+\cdots+\varepsilon_{n} x_{j}^{n}\right)\right)_{j=1}^{\infty} \in Y(F)$ para toda escolha de sinais $\varepsilon_{1}, \ldots, \varepsilon_{n}= \pm 1$. Aplicando a Fórmula de Polarização e o fato de $Y(F)$ também ser um espaço vetorial, obtemos

$$
\begin{aligned}
\left(\check{P}\left(x_{j}^{1}, \ldots, x_{j}^{n}\right)\right)_{j=1}^{\infty} & =\left(\frac{1}{n ! 2^{n}} \sum_{\varepsilon_{j}= \pm 1} \varepsilon_{1} \cdots \varepsilon_{n} P\left(\varepsilon_{1} x_{j}^{1}+\cdots+\varepsilon_{n} x_{j}^{n}\right)\right)_{j=1}^{\infty} \\
& =\frac{1}{n ! 2^{n}} \sum_{\varepsilon_{j}= \pm 1} \varepsilon_{1} \cdots \varepsilon_{n}\left(P\left(\varepsilon_{1} x_{j}^{1}+\cdots+\varepsilon_{n} x_{j}^{n}\right)\right)_{j=1}^{\infty} \in Y(F) .
\end{aligned}
$$

Isso prova que $\check{P} \in \mathcal{L}_{X ; Y}\left({ }^{n} E ; F\right)$.

$(d) \Longrightarrow(c)$ Essa implicação é óbvia.

$(b) \Longrightarrow(d)$ Por hipótese existe $A \in \mathcal{L}_{X ; Y}\left({ }^{n} E ; F\right)$ tal que $\widehat{A}=P$. Por [11, Proposition 2.4], o operador $n$-linear induzido $\widetilde{A}: X(E)^{n} \longrightarrow Y(F)$ é contínuo. Daí, seu polinômio associado $\widehat{(\widetilde{A})}: X(E) \longrightarrow Y(F)$ também é contínuo. Basta provar que $\widetilde{P}=\widehat{(\widetilde{A})}$. Com efeito, para toda sequência $\left(x_{j}\right)_{j=1}^{\infty} \in X(E)$,

$\widehat{(\widetilde{A})}\left(\left(x_{j}\right)_{j=1}^{\infty}\right)=\widetilde{A}\left(\left(x_{j}\right)_{j=1}^{\infty}, \ldots,\left(x_{j}\right)_{j=1}^{\infty}\right)=\left(A\left(x_{j}, \ldots, x_{j}\right)\right)_{j=1}^{\infty}=\left(P\left(x_{j}\right)\right)_{j=1}^{\infty}=\widetilde{P}\left(\left(x_{j}\right)_{j=1}^{\infty}\right)$.

Em relação às normas, a igualdade $\widetilde{P}=\widehat{(\widetilde{A})}$ também implica que

$$
\|\widetilde{P}\|=\|\widehat{(\widetilde{A})}\| \leq\|\widetilde{A}\|=\|A\|_{X ; Y}
$$


para toda $A \in \mathcal{L}_{X ; Y}\left({ }^{n} E ; F\right)$ tal que $\widehat{A}=P$. Segue que

$$
\|\widetilde{P}\| \leq \inf \left\{\|A\|_{X ; Y}: A \in \mathcal{L}_{X ; Y} \text { e } \widehat{A}=P\right\} \leq\|\check{P}\|_{X ; Y},
$$

onde a última desigualdade vem de $(a)$.

Da unicidade do operador simétrico $\check{P}$ associado a $P$, sabemos que $A_{s}=\check{P}$. Pela Proposição 5.1.10 sabemos que o hiper-ideal $\mathcal{L}_{X ; Y}$ é fortemente simétrico, $\operatorname{logo} A_{s} \in \mathcal{L}_{X ; Y}$ e $\left\|A_{\sigma}\right\|_{X ; Y}=\|A\|_{X ; Y}$ para toda permutação $\sigma \in S_{n}$. Portanto,

$$
\|\check{P}\|_{X ; Y}=\left\|A_{s}\right\|_{X ; Y}=\left\|\frac{1}{n !} \cdot \sum_{\sigma \in S_{n}} A_{\sigma}\right\|_{X ; Y} \leq \frac{1}{n !} \cdot \sum_{\sigma \in S_{n}}\left\|A_{\sigma}\right\|_{X ; Y}=\|A\|_{X ; Y}
$$

para toda $A \in \mathcal{L}_{X ; Y}\left({ }^{n} E ; F\right)$ tal que $\widehat{A}=P$. Segue que $\inf \left\{\|A\|_{X ; Y}: A \in \mathcal{L}_{X ; Y}\right.$ e $\widehat{A}=$ $P\}=\|\check{P}\|_{X ; Y}$.

Para a desigualdade restante, note que acima provamos que $\widetilde{P}=\widehat{(\widetilde{A})}$ para toda $A \in \mathcal{L}_{X ; Y}\left({ }^{n} E ; F\right)$ tal que $\widehat{A}=P$. Como $\widehat{\ddot{P}}=P$ e, por $($ a $), \check{P} \in \mathcal{L}_{X ; Y}\left({ }^{n} E ; F\right)$, segue que $\widetilde{P}=\widehat{(\widetilde{P})}$. É fácil observar que, uma vez que $\check{P}$ é simétrico, seu operador $n$-linear induzido $\widetilde{\widetilde{P}}$ também é simétrico. Pela Proposição 1.6.2,

$$
\|\check{P}\|_{X ; Y}=\|\widetilde{\widetilde{P}}\| \leq \frac{n^{n}}{n !} \cdot\|\widehat{(\widetilde{\widetilde{P}})}\|=\frac{n^{n}}{n !} \cdot\|\widetilde{P}\| .
$$

Agora podemos definir polinômios homogêneos $(X ; Y)$-somantes.

Definição 5.2.2. Sejam $X$ e $Y$ classes de sequências. Dizemos que um polinômio $P \in$ $\mathcal{P}\left({ }^{n} E ; F\right)$ é $(X ; Y)$-somante se $P$ satisfaz as condições equivalentes do teorema anterior. Denotaremos o conjunto de tais polinômios por $\mathcal{P}_{X ; Y}\left({ }^{n} E ; F\right)$ e definimos, para cada $P \in$ $\mathcal{P}_{X ; Y}\left({ }^{n} E ; F\right)$,

$$
\|P\|_{X ; Y ; 1}=\|\widetilde{P}\| \text { e }\|P\|_{X ; Y ; 2}=\|\check{P}\|_{X ; Y}
$$

Relembre na Definição 4.1.2 o conceito de hiper-ideal de polinômios.

Teorema 5.2.3. Sejam $X$ uma classe de sequências multilinearmente estável e $Y$ uma classe de sequências linearmente estável tais que $X(\mathbb{K})^{n} \hookrightarrow Y(\mathbb{K})$ para todo $n \in \mathbb{N}$. Então $\left(\mathcal{P}_{X ; Y},\|\cdot\|_{X ; Y ; 1}\right)$ e $\left(\mathcal{P}_{X ; Y},\|\cdot\|_{X ; Y ; 2}\right)$ são $\left(\frac{n^{n}}{n !}\right)_{n=1}^{\infty}$-hiper-ideais de Banach de polinômios homogêneos. 
Demonstração. Do Teorema 5.1.7 sabemos que $\left(\mathcal{L}_{X ; Y},\|\cdot\|_{X ; Y}\right)$ é um hiper-ideal de Banach de operadores multilineares. Pelo Teorema 5.2.1, um polinômio $P$ pertence a $\mathcal{P}_{X ; Y}$ se, e somente se, existe $A$ em $\mathcal{L}_{X ; Y}$ tal que $\widehat{A}=P$. Segue de [21, Theorem 4.3] que $\left(\mathcal{P}_{X ; Y}, \| \cdot\right.$ $\left.\|_{X ; Y ; 2}\right)$ é $\left(\frac{n^{n}}{n !}\right)_{n=1}^{\infty}$-hiper-ideal de Banach de polinômios.

Em vista do que acabamos de provar, basta verificar as questões relativas à norma $\|\cdot\|_{X ; Y ; 1}$. Os axiomas de norma são imediatos. Pelo Teorema 5.2.1 sabemos que as normas $\|\cdot\|_{X ; Y ; 1}$ e $\|\cdot\|_{X ; Y ; 2}$ são equivalentes em cada componente $\mathcal{P}_{X ; Y}\left({ }^{n} E ; F\right)$. Pelo que já foi provado sabemos que esse espaço é completo com a norma $\|\cdot\|_{X ; Y ; 2}$, portanto também é completo com a norma $\|\cdot\|_{X ; Y ; 1}$.

Provemos que $\left\|\hat{I}_{n}: \mathbb{K} \longrightarrow \mathbb{K}, \hat{I}_{n}(\lambda)=\lambda^{n}\right\|_{X ; Y ; 1}=1$ para todo $n$. Note que

$$
\begin{aligned}
\left\|\hat{I}_{n}\right\|_{X ; Y ; 1} & =\left\|\tilde{\hat{I}}_{n}\right\|=\sup \left\{\left\|\tilde{\hat{I}}_{n}\left(\left(\lambda_{j}\right)_{j=1}^{\infty}\right)\right\|_{Y(\mathbb{K})}:\left(\lambda_{j}\right)_{j=1}^{\infty} \in B_{X(\mathbb{K})}\right\} \\
& =\sup \left\{\left\|\left(\hat{I}_{n}\left(\lambda_{j}\right)\right)_{j=1}^{\infty}\right\|_{Y(\mathbb{K})}:\left(\lambda_{j}\right)_{j=1}^{\infty} \in B_{X(\mathbb{K})}\right\} \\
& =\sup \left\{\left\|\left(\lambda_{j}^{n}\right)_{j=1}^{\infty}\right\|_{Y(\mathbb{K})}:\left(\lambda_{j}\right)_{j=1}^{\infty} \in B_{X(\mathbb{K})}\right\} .
\end{aligned}
$$

Por um lado, tomando $e_{1}=(1,0,0,0 \ldots$,$) , da definição de classes de sequências temos$ $\left\|e_{1}\right\|_{X(\mathbb{K})}=\left\|e_{1}\right\|_{Y(\mathbb{K})}=1$. Segue da expressão acima que $\left\|\hat{I}_{n}\right\|_{X ; Y ; 1} \geq 1$. Por outro lado, combinando a expressão acima com a condição de compatibilidade $X(\mathbb{K})^{n} \hookrightarrow Y(\mathbb{K})$ temos

$$
\left\|\hat{I}_{n}\right\|_{X ; Y ; 1} \leq \sup \left\{\left\|\left(\lambda_{j}\right)_{j=1}^{\infty}\right\|_{Y(\mathbb{K})}^{n}:\left(\lambda_{j}\right)_{j=1}^{\infty} \in B_{X(\mathbb{K})}\right\} \leq 1 .
$$

Resta verificar a desigualdade de hiper-ideal. Para isso sejam $n, m \in \mathbb{N}$, espaços de Banach $E, F, G$ e $H, P \in \mathcal{P}_{X ; Y}\left({ }^{n} E ; F\right), Q \in \mathcal{P}\left({ }^{m} G ; E\right)$ e $t \in \mathcal{L}(F ; H)$. Já sabemos que to $P \circ Q \in \mathcal{P}_{X ; Y}\left({ }^{m n} G ; H\right)$. É fácil perceber que $\widetilde{Q}: X(G) \longrightarrow X(E)$ é o polinômio associado ao operador simétrico $\widetilde{Q}: X(G)^{m} \longrightarrow X(E)$. Isso justifica a primeira desigualdade a seguir:

$$
\|\widetilde{Q}\| \leq\|\widetilde{Q}\|=\|\check{Q}\| \leq \frac{m^{m}}{m !}\|Q\|
$$

onde a igualdade segue da estabilidade multilinear de $X$. De forma análoga ao que foi feito na demonstração do Teorema 5.1.7, prova-se que $[t \circ P \circ Q]^{\sim}=\widetilde{t} \circ \widetilde{P} \circ \widetilde{Q}$. Daí,

$$
\begin{aligned}
\|t \circ P \circ Q\|_{X ; Y ; 1} & =\left\|[t \circ P \circ Q]^{\sim}\right\|=\|\widetilde{t} \circ \widetilde{P} \circ \widetilde{Q}\| \leq\|\widetilde{t}\| \cdot\|\widetilde{P}\| \cdot\|\widetilde{Q}\|^{m} \\
& =\|t\| \cdot\|P\|_{X ; Y ; 1} \cdot\|\widetilde{Q}\|^{m} \leq\left(\frac{m^{m}}{m !}\right)^{m}\|t\| \cdot\|P\|_{X ; Y ; 1} \cdot\|Q\|^{m} .
\end{aligned}
$$


A demonstração acima deixa clara a condição que deve ser imposta à classe de sequências $X$ para que $\left(\mathcal{P}_{X ; Y},\|\cdot\|_{X ; Y ; 1}\right)$ seja um hiper-ideal de Banach de polinômios com constantes iguais a 1. Faremos isso a seguir.

Definição 5.2.4. Dizemos que uma classe de sequências $X$ é polinomialmente estável se, dados $n \in \mathbb{N}$, espaços de Banach $E, F$ e $P \in \mathcal{P}\left({ }^{n} E ; F\right)$, tem-se $\left(P\left(x_{j}\right)\right)_{j=1}^{\infty} \in X(F)$ sempre que $\left(x_{j}\right)_{j=1}^{\infty} \in X(E)$ e que $\widetilde{P}: X(E) \longrightarrow X(F)$ é um polinômio $n$-homogêneo contínuo com $\|\widetilde{P}\|=\|P\|$.

Para dar um exemplo útil, precisamos do seguinte lema.

Lema 5.2.5. Sejam $X$ e $Y$ classes de sequências linearmente estáveis. Então $\|P\| \leq$ $\|P\|_{X ; Y ; 1}$ para todo $P \in \mathcal{P}_{X ; Y}\left({ }^{n} E ; F\right)$.

Demonstração. Para cada $x \in E$, por $[11$, Lemma 3.4(a) $]$ sabemos que $\|(x, 0,0, \ldots)\|_{X(E)}=$ $\|x\|$ e $\|(P(x), 0,0, \ldots)\|_{Y(F)}=\|P(x)\|$. Sabemos que $\widetilde{P}: X(E) \longrightarrow Y(F)$ é um polinômio $n$-homogêneo contínuo, logo

$$
\begin{aligned}
\|P(x)\| & =\|(P(x), 0,0, \ldots)\|_{Y(F)}=\|\widetilde{P}((x, 0,0, \ldots))\|_{Y(F)} \leq\|\widetilde{P}\| \cdot\|(x, 0,0, \ldots)\|_{X(E)}^{n} \\
& =\|P\|_{X ; Y ; 1} \cdot\|x\|^{n},
\end{aligned}
$$

donde segue a desigualdade desejada.

Exemplo 5.2.6. Vejamos que, para todo $1 \leq p<+\infty$, a classe $\ell_{p}(\cdot)$ é polinomialmente estável. Dados $P \in \mathcal{P}\left({ }^{n} E ; F\right)$ e $\left(x_{j}\right)_{j=1}^{\infty} \in \ell_{p}(E)$,

$$
\begin{aligned}
\left\|\left(P\left(x_{j}\right)\right)_{j=1}^{\infty}\right\|_{p} & =\left(\sum_{j=1}^{\infty}\left\|P\left(x_{j}\right)\right\|^{p}\right)^{1 / p} \leq\|P\| \cdot\left(\sum_{j=1}^{\infty}\left\|x_{j}\right\|^{n p}\right)^{1 / p}=\|P\| \cdot\left\|\left(x_{j}\right)_{j=1}^{\infty}\right\|_{n p}^{n} \\
& \leq\|P\| \cdot\left\|\left(x_{j}\right)_{j=1}^{\infty}\right\|_{p}^{n},
\end{aligned}
$$

pois $p \leq n p$. Isso mostra que $\left(P\left(x_{j}\right)\right)_{j=1}^{\infty} \in \ell_{p}(F)$. Pelo Teorema 5.2.1, $\widetilde{P}: \ell_{p}(E) \longrightarrow \ell_{p}(F)$ é um polinômio $n$-homogêneo bem definido e contínuo. Da desigualdade acima temos

$$
\left\|\widetilde{P}\left(\left(x_{j}\right)_{j=1}^{\infty}\right)\right\|_{p}=\left\|\left(P\left(x_{j}\right)\right)_{j=1}^{\infty}\right\|_{p} \leq\|P\| \cdot\left\|\left(x_{j}\right)_{j=1}^{\infty}\right\|_{p}^{n},
$$

para toda $\left(x_{j}\right)_{j=1}^{\infty} \in \ell_{p}(E)$, o que prova que $\|\widetilde{P}\| \leq\|P\|$. Tomando $X=Y=\ell_{p}(\cdot)$ no Lema 5.2.5 obtemos $\|P\| \leq\|P\|_{\ell_{p}(\cdot) ; \ell_{p}(\cdot), 1}=\|\widetilde{P}\|$.

Teorema 5.2.7. Sejam $X$ uma classe de sequências polinomialmente estável e $Y$ uma classe de sequências linearmente estável tais que $X(\mathbb{K})^{n} \hookrightarrow Y(\mathbb{K})$ para todo $n \in \mathbb{N}$. Então $\left(\mathcal{P}_{X ; Y},\|\cdot\|_{X ; Y ; 1}\right)$ é um hiper-ideal de Banach de polinômios homogêneos. 
Demonstração. De acordo com o Teorema 5.2.3 basta mostrar que a desigualdade de hiper-ideal vale com constantes iguais a 1 . Sejam $n, m \in \mathbb{N}$, espaços de Banach $E, F, G$ e $H, P \in \mathcal{P}_{X ; Y}\left({ }^{n} E ; F\right), Q \in \mathcal{P}\left({ }^{m} G ; E\right)$ e $t \in \mathcal{L}(F ; H)$. Da estabilidade polinomial de $X$ sabemos que $\widetilde{Q}: X(G) \longrightarrow X(E)$ é um polinômio $m$-homogêneo contínuo e $\|\widetilde{Q}\|=\|Q\|$. Sabemos também que $[t \circ P \circ Q]^{\sim}=\widetilde{t} \circ \widetilde{P} \circ \widetilde{Q}$, daí,

$\|t \circ P \circ Q\|_{X ; Y ; 1}=\left\|[t \circ P \circ Q]^{\sim}\right\|=\|\widetilde{t} \circ \widetilde{P} \circ \widetilde{Q}\| \leq\|\widetilde{t}\| \cdot\|\widetilde{P}\| \cdot\|\widetilde{Q}\|^{m}=\|t\| \cdot\|P\|_{X ; Y ; 1} \cdot\|Q\|^{m}$.

Veremos a seguir que classes de sequências também geram ideais bilaterais de polinômios, noção introduzida em [23].

Definição 5.2.8. Seja $\left(C_{n}, K_{n}\right)_{n=1}^{\infty}$ uma sequência de pares de números reais positivos com $C_{n}, K_{n} \geq 1$ para todo $n$ e $C_{1}=K_{1}=1$. Dizemos que um ideal de Banach de polinômios $\left(\mathcal{Q},\|\cdot\|_{\mathcal{Q}}\right)$ é um $\left(C_{n}, K_{n}\right)_{n=1}^{\infty}$-ideal bilateral de Banach de polinômios se satisfaz a seguinte propriedade: dados $m, n, r \in \mathbb{N}$, espaços de Banach $E, F, G$ e $H$, se $P \in \mathcal{Q}\left({ }^{n} E ; F\right)$, $Q \in \mathcal{P}\left({ }^{m} G ; E\right)$ e $R \in \mathcal{P}\left({ }^{r} F ; H\right)$, então $R \circ P \circ Q \in \mathcal{Q}\left({ }^{r m n} G ; H\right)$ e

$$
\|R \circ P \circ Q\|_{\mathcal{Q}} \leq K_{r} \cdot C_{m}^{r n} \cdot\|R\| \cdot\|P\|_{\mathcal{Q}}^{r} \cdot\|Q\|^{r n}
$$

Precisaremos do seguinte resultado:

Proposição 5.2.9. [23, Proposição 4.2] Seja $\left(\mathcal{H},\|\cdot\|_{\mathcal{H}}\right)$ um hiper-ideal normado (Banach) de operadores multilineares tal que $\mathcal{L} \circ \mathcal{H} \hookrightarrow \mathcal{H}$, isto é, tal que para todo operador $A \in$ $\mathcal{L}\left(E_{1}, \ldots, E_{n} ; F\right)$ podemos encontrar números $m$ e $r$ com $m r=n$, um espaço de Banach $G$, operadores multilineares $B_{1} \in \mathcal{H}\left(E_{1}, \ldots, E_{m} ; G\right), \ldots, B_{r} \in \mathcal{H}\left(E_{(r-1) \cdot m+1}, \ldots, E_{n} ; G\right)$ e um operador multilinear simétrico $C \in \mathcal{L}\left({ }^{r} G ; F\right)$ tais que

$$
A=C \circ\left(B_{1}, \ldots, B_{r}\right)
$$

e, neste caso, escrevemos,

$$
\|A\|_{\mathcal{L} \circ \mathcal{H}}=\inf \left\{\|C\| \cdot\left\|B_{1}\right\|_{\mathcal{H}} \cdots\left\|B_{r}\right\|_{\mathcal{H}}\right\}
$$

onde o ínfimo é tomado sobre todas as fatorações de $A$ como em (5.3). Então $\left(\mathcal{P}^{\mathcal{H}},\|\cdot\|_{\mathcal{P}^{\mathcal{H}}}\right)$ é um $\left(\frac{n^{n}}{n !}, \frac{n^{n}}{n !}\right)$-ideal bilateral normado (Banach) de polinômios.

Teorema 5.2.10. Sejam $X$ e $Y$ classes de sequências multilinearmente estáveis tais que $X(\mathbb{K})^{n} \hookrightarrow Y(\mathbb{K})$ para todo $n \in \mathbb{N}$. Então $\left(\mathcal{P}_{X ; Y},\|\cdot\|_{X ; Y ; 2}\right)$ é um $\left(\frac{n^{n}}{n !}, \frac{n^{n}}{n !}\right)$-ideal bilateral de Banach de polinômios. 
Demonstração. Do Teorema 5.1.7 sabemos que $\left(\mathcal{L}_{X ; Y},\|\cdot\|_{X ; Y}\right)$ é um hiper-ideal de Banach de operadores multilineares. Vejamos que esse hiper-ideal satisfaz a hipótese da proposição anterior. Para isso sejam $A \in \mathcal{L}\left(E_{1}, \ldots, E_{n} ; F\right), m, r \in \mathbb{N}$ tais que $m r=n, G$ um espaço de Banach, $B_{1} \in \mathcal{L}_{X ; Y}\left(E_{1}, \ldots, E_{m} ; G\right), \ldots, B_{r} \in \mathcal{L}_{X ; Y}\left(E_{(r-1) m+1}, \ldots, E_{n} ; G\right)$ e $C \in \mathcal{L}\left({ }^{r} G ; F\right)$ simétrica tais que $A=C \circ\left(B_{1}, \ldots, B_{r}\right)$. Devemos provar que

$$
A \in \mathcal{L}_{X ; Y}\left(E_{1}, \ldots, E_{n} ; F\right) \text { e }\|A\|_{X ; Y} \leq\|C\| \cdot\left\|B_{1}\right\|_{X ; Y} \cdots\left\|B_{r}\right\|_{X ; Y} .
$$

Dadas sequências $\left(x_{j}^{k}\right)_{j=1}^{\infty} \in X\left(E_{k}\right), k=1, \ldots, n$, temos

$$
\left(B_{1}\left(x_{j}^{1}, \ldots, x_{j}^{m}\right)\right)_{j=1}^{\infty}, \ldots,\left(B_{r}\left(x_{j}^{(r-1) m+1}, \ldots, x_{j}^{n}\right)\right)_{j=1}^{\infty} \in Y(G)
$$

$\mathrm{e}$

$$
\begin{gathered}
\left\|\widetilde{B_{1}}: X\left(E_{1}\right) \times \cdots \times X\left(E_{m}\right) \longrightarrow Y(G)\right\|=\left\|B_{1}\right\|, \ldots, \\
\left\|\widetilde{B_{r}}: X\left(E_{(r-1) m+1}\right) \times \cdots \times X\left(E_{n}\right) \longrightarrow G\right\|=\left\|B_{r}\right\| .
\end{gathered}
$$

Da estabilidade multilinear de $Y$ segue que

$$
\begin{aligned}
\left(A\left(x_{j}^{1}, \ldots, x_{j}^{n}\right)\right)_{j=1}^{\infty} & =\left(C \circ\left(B_{1}, \ldots, B_{r}\right)\left(x_{j}^{1}, \ldots, x_{j}^{n}\right)\right)_{j=1}^{\infty} \\
& =\left(C\left(B_{1}\left(x_{j}^{1}, \ldots, x_{j}^{m}\right), \ldots, B_{r}\left(x_{j}^{(r-1) m+1}, \ldots, x_{j}^{n}\right)\right)\right)_{j=1}^{\infty} \in Y(F),
\end{aligned}
$$

o que prova que $A \in \mathcal{L}_{X ; Y}\left(E_{1}, \ldots, E_{n} ; F\right)$ e, mais ainda,

$$
\begin{aligned}
\|A\|_{X ; Y} & =\left\|\widetilde{A}: X\left(E_{1}\right) \times \cdots \times X\left(E_{n}\right) \longrightarrow Y(F)\right\| \\
& =\left\|\left[C \circ\left(B_{1}, \ldots, B_{r}\right)\right]^{\sim}\right\| \\
& =\left\|\widetilde{C} \circ\left(\widetilde{B_{1}}, \ldots, \widetilde{B_{r}}\right)\right\| \\
& \leq\left\|\widetilde{C}: Y(G)^{r} \longrightarrow Y(F)\right\| \cdots\left\|\widetilde{B_{1}}\right\| \cdots\left\|\widetilde{B_{r}}\right\| \\
& =\|C\| \cdot\left\|B_{1}\right\|_{X ; Y} \cdots\left\|B_{r}\right\|_{X ; Y} .
\end{aligned}
$$

Uma vez satisfeita a hipótese da Proposição 5.2.9 o desejado segue desse resultado e do Teorema 5.2.1.

Com a estabilidade polinomial podemos ir um pouco mais longe.

Teorema 5.2.11. Sejam $X$ e $Y$ classes de sequências com $X$ polinomialmente estável e $Y$ multilinearmente estável tais que $X(\mathbb{K})^{n} \hookrightarrow Y(\mathbb{K})$ para todo $n \in \mathbb{N}$. Então $\left(\mathcal{P}_{X ; Y},\|\cdot\|_{X ; Y ; 1}\right)$ é um $\left(1, \frac{n^{n}}{n !}\right)_{n=1}^{\infty}$-ideal bilateral de Banach de polinômios. 
Demonstração. Pelo teorema anterior basta provar que a desigualdade de ideal bilateral vale com constantes $\left(1, \frac{n^{n}}{n !}\right)_{n=1}^{\infty}$. Sejam $m, n, r \in \mathbb{N}, P \in \mathcal{P}_{X ; Y}\left({ }^{n} E ; F\right), Q \in \mathcal{P}\left({ }^{m} G ; E\right)$ e $R \in \mathcal{P}\left({ }^{r} F ; H\right)$. Pelo Teorema 5.2 .7 sabemos que $\|P \circ Q\|_{X ; Y ; 1} \leq\|P\|_{X ; Y ; 1} \cdot\|Q\|^{n}$. Denotando $\widetilde{R}: Y(F) \longrightarrow Y(H),[P \circ Q]^{\sim}: X(G) \longrightarrow Y(F)$ e $[R \circ P \circ Q]^{\sim}: X(G) \longrightarrow Y(H)$, temos $[R \circ P \circ Q]^{\sim}=\widetilde{R} \circ[P \circ Q]^{\sim}$. Conforme visto na demonstração do Teorema 5.2.1, $\widetilde{R}=\widehat{(\widetilde{\widetilde{R}})}$, e portanto da estabilidade multilinear de $Y$ segue que

$$
\begin{aligned}
\|R \circ P \circ Q\|_{X ; Y ; 1} & =\left\|[R \circ P \circ Q]^{\sim}\right\|=\left\|\widetilde{R} \circ[P \circ Q]^{\sim}\right\| \leq\|\widetilde{R}\| \cdot\|P\|_{X ; Y ; 1}^{r} \cdot\|Q\|^{r n} \\
& =\|\widehat{(\widetilde{\widetilde{R}})}\| \cdot\|P\|_{X ; Y ; 1}^{r} \cdot\|Q\|^{r n} \leq\|\widetilde{\widetilde{R}}\| \cdot\|P\|_{X ; Y ; 1}^{r} \cdot\|Q\|^{r n} \\
& =\|\check{R}\| \cdot\|P\|_{X ; Y ; 1}^{r} \cdot\|Q\|^{r n} \leq \frac{r^{r}}{r !} \cdot\|R\| \cdot\|P\|_{X ; Y ; 1}^{r} \cdot\|Q\|^{r n} .
\end{aligned}
$$

Como era de se esperar, a melhor informação é obtida quando as duas classes de sequências são polinomialmente estáveis.

Teorema 5.2.12. Sejam $X$ e $Y$ classes de sequências polinomialmente estáveis tais que $X(\mathbb{K})^{n} \hookrightarrow Y(\mathbb{K})$ para todo $n \in \mathbb{N}$. Então $\left(\mathcal{P}_{X ; Y},\|\cdot\|_{X ; Y ; 1}\right)$ é um ideal bilateral de Banach de polinômios.

Demonstração. Pelo Teorema 5.2.10 basta provar que a desigualdade de ideal bilateral vale com constantes iguais a 1 . Sejam $m, n, r \in \mathbb{N}, P \in \mathcal{P}_{X ; Y}\left({ }^{n} E ; F\right), Q \in \mathcal{P}\left({ }^{m} G ; E\right)$ e $R \in \mathcal{P}\left({ }^{r} F ; H\right)$. Temos $\|P\|_{X ; Y ; 1}=\|\widetilde{P}: X(E) \longrightarrow Y(F)\|$ por definição, $\| \widetilde{Q}: X(G) \longrightarrow$ $X(E)\|=\| Q \|$ e $\|\widetilde{R}: Y(F) \longrightarrow Y(H)\|=\|R\|$ pela estabilidade polinomial das classes $X$ e $Y$. Portanto,

$$
\begin{aligned}
\|R \circ P \circ Q\|_{X ; Y ; 1} & =\left\|[R \circ P \circ Q]^{\sim}\right\|=\|\widetilde{R} \circ \widetilde{P} \circ \widetilde{Q}\| \\
& \leq\|\widetilde{R}\| \cdot\|\widetilde{P}\|^{r} \cdot\|\widetilde{Q}\|^{r n}=\|R\| \cdot\|P\|_{X ; Y ; 1}^{r} \cdot\|Q\|^{r n}
\end{aligned}
$$

Assim como fizemos no caso multilinear, mostraremos alguns exemplos de ideais de polinômios que têm sido estudados e que, na verdade, são hiper-ideais ou ideais bilaterais; e também uma nova classe. Para evitar repetições, dessa vez seremos mais breves. 
Exemplos 5.2.13. Aplicando os teoremas provados nesta seção para classes de sequências que vimos nos Exemplos 5.1.3 e 5.2.6 serem linearmente estáveis, multilinearmente estáveis e polinomialmente estáveis, obtemos os seguintes exemplos:

(a) A classe $\mathcal{P}_{\ell_{1}^{w}(\cdot) ; \ell_{1}(\cdot)}$ dos polinômios homogêneos absolutamente somantes é um $\left(\frac{n^{n}}{n !}\right)_{n=1}^{\infty}$ hiper-ideal de Banach de polinômios com a norma $\|\cdot\|_{X ; Y ; 1}$ e um $\left(\frac{n^{n}}{n !}, \frac{n^{n}}{n !}\right)_{n=1}^{\infty}$-ideal bilateral de Banach de polinômios com a norma $\|\cdot\|_{X ; Y ; 2}$.

(b) A classe $\mathcal{P}_{\ell_{1}^{w}(\cdot) ; \ell_{1}^{w}(\cdot)}$ dos polinômios homogêneos fracamente somantes é um $\left(\frac{n^{n}}{n !}\right)_{n=1}^{\infty}$ hiper-ideal de Banach de polinômios com a norma $\|\cdot\|_{X ; Y ; 1}$ e um $\left(\frac{n^{n}}{n !}, \frac{n^{n}}{n !}\right)_{n=1}^{\infty}$-ideal bilateral de Banach de polinômios com a norma $\|\cdot\|_{X ; Y ; 2}$.

(c) Para $1 \leq p \leq 2$, a classe $\mathcal{P}_{\ell_{p}(\cdot) ; \operatorname{Rad}(\cdot)}$ dos polinômios homogêneos de tipo $p$ é um $\left(1, \frac{n^{n}}{n !}\right)_{n=1}^{\infty}$-ideal bilateral de Banach de polinômios com a norma $\|\cdot\|_{X ; Y ; 1}$ e um $\left(\frac{n^{n}}{n !}, \frac{n^{n}}{n !}\right)_{n=1}^{\infty}$ ideal bilateral de Banach de polinômios com a norma $\|\cdot\|_{X ; Y ; 2}$.

(d) Para $2 \leq q<+\infty$, a classe $\mathcal{P}_{\operatorname{Rad}(\cdot) ; \ell_{q}(\cdot)}$ dos polinômios homogêneos de cotipo $q$ é um $\left(\frac{n^{n}}{n !}\right)_{n=1}^{\infty}$-hiper-ideal de Banach de polinômios com a norma $\|\cdot\|_{X ; Y ; 1}$ e um $\left(\frac{n^{n}}{n !}, \frac{n^{n}}{n !}\right)_{n=1}^{\infty}$-ideal bilateral de Banach de polinômios com a norma $\|\cdot\|_{X ; Y ; 2}$.

(e) A classe $\mathcal{P}_{\operatorname{Rad}(\cdot) ; \ell_{2}\langle\cdot\rangle}$ dos polinômios homogêneos Cohen quase somantes é $\left(\frac{n^{n}}{n !}\right)_{n=1}^{\infty}$-hiperideal de Banach de polinômios com a norma $\|\cdot\|_{X ; Y ; 1}$ e um $\left(\frac{n^{n}}{n !}, \frac{n^{n}}{n !}\right)_{n=1}^{\infty}$-ideal bilateral de Banach de polinômios com a norma $\|\cdot\|_{X ; Y ; 2}$.

(f) Para $1 \leq p<+\infty$, a classe $\mathcal{P}_{\ell_{p}(\cdot) ; \ell_{p}\langle\cdot\rangle}$ dos polinômios associados ao multi-ideal $\mathcal{L}_{\ell_{p}(\cdot) ; \ell_{p}\langle\cdot\rangle}$ do Exemplo 5.1.16 é um $\left(1, \frac{n^{n}}{n !}\right)_{n=1}^{\infty}$-ideal bilateral de Banach de polinômios com a norma $\|\cdot\|_{X ; Y ; 1}$ e um $\left(\frac{n^{n}}{n !}, \frac{n^{n}}{n !}\right)_{n=1}^{\infty}$-ideal bilateral de Banach de polinômios com a norma $\|\cdot\|_{X ; Y ; 2}$.

\subsection{Comparação com os ideais de composição}

Dadas classes de sequências linearmente estáveis $X$ e $Y$, seja $\Pi_{X ; Y}$ a componente linear do multi-ideal $\mathcal{L}_{X ; Y}$, ou equivalentemente, do ideal de polinômios $\mathcal{P}_{X ; Y}$, isto é, $\Pi_{X ; Y}$ é o ideal de Banach dos operadores lineares $u: E \longrightarrow F$ tais que $\left(u\left(x_{j}\right)\right)_{j=1}^{\infty} \in Y(F)$ sempre que $\left(x_{j}\right)_{j=1}^{\infty} \in X(E)$.

Um dos objetivos deste capítulo foi obter condições sob as quais $\mathcal{L}_{X ; Y}$ é um hiper-ideal. A questão é que já é conhecido que, por ser um ideal de composição, $\Pi_{X ; Y} \circ \mathcal{L}$ é um hiperideal (Teorema 1.5.2). Assim, se fosse verdade que $\mathcal{L}_{X ; Y}=\Pi_{X ; Y} \circ \mathcal{L}$, não estaríamos 
construindo novos hiper-ideais, estaríamos apenas revisitando hiper-ideais conhecidos. Veremos nesta seção que esse não é o caso, e portanto os ideais $\mathcal{L}_{X ; Y}$ são, de fato, hiperideais que não haviam sido estudados antes.

De acordo com o Teorema 5.1.7, $\mathcal{L}_{X ; Y}$ é um hiper-ideal se $X$ for multilinearmente estável. É esse então o caso que devemos tratar. Começamos mostrando que, neste caso, uma inclusão vale, com a respectiva desigualdade de normas.

Proposição 5.3.1. Sejam X e $Y$ classes de sequências com $X$ multilinearmente estável. Então $\Pi_{X ; Y} \circ \mathcal{L} \subseteq \mathcal{L}_{X ; Y}$ e $\|\cdot\|_{X ; Y} \leq\|\cdot\|_{\Pi_{X ; Y} \circ \mathcal{L} \text {. }}$

Demonstração. Dado um operador $A \in \Pi_{X ; Y} \circ \mathcal{L}\left(E_{1}, \ldots, E_{n} ; F\right)$, existem um espaço de Banach $G$, um operador $n$-linear $B \in \mathcal{L}\left(E_{1}, \ldots, E_{n} ; G\right)$ e um operador linear $u \in$ $\Pi_{X ; Y}(G ; F)$ tais que $A=u \circ B$. Para todas sequências $\left(x_{j}^{k}\right)_{j=1}^{\infty} \in X\left(E_{k}\right), k=1, \ldots, n$, $\left(B\left(x_{j}^{1}, \ldots, x_{j}^{n}\right)\right)_{j=1}^{\infty} \in X(G)$ pois $X$ é multilinearmente estável. E como $u$ é $(X ; Y)$ somante,

$$
\left(A\left(x_{j}^{1}, \ldots, x_{j}^{n}\right)\right)_{j=1}^{\infty}=\left(u\left(B\left(x_{j}^{1}, \ldots, x_{j}^{n}\right)\right)\right)_{j=1}^{\infty} \in Y(F),
$$

provando que $A \in \mathcal{L}_{X ; Y}\left(E_{1}, \ldots, E_{n} ; F\right)$. Além disso,

$$
\begin{aligned}
\|A\|_{X ; Y} & =\|\widetilde{A}\|=\sup \left\{\left\|\left(A\left(x_{j}^{1}, \ldots, x_{j}^{n}\right)\right)_{j=1}^{\infty}\right\|_{Y(F)}:\left(x_{j}^{k}\right)_{j=1}^{\infty} \in B_{X\left(E_{k}\right)}, k=1, \ldots, n\right\} \\
& =\sup \left\{\left\|\left(u\left(B\left(x_{j}^{1}, \ldots, x_{j}^{n}\right)\right)\right)_{j=1}^{\infty}\right\|_{Y(F)}:\left(x_{j}^{k}\right)_{j=1}^{\infty} \in B_{X\left(E_{k}\right)}, k=1, \ldots, n\right\} \\
& =\sup \left\{\left\|\widetilde{u}\left(\left(B\left(x_{j}^{1}, \ldots, x_{j}^{n}\right)\right)_{j=1}^{\infty}\right)\right\|_{Y(F)}:\left(x_{j}^{k}\right)_{j=1}^{\infty} \in B_{X\left(E_{k}\right)}, k=1, \ldots, n\right\} \\
& \leq\|\widetilde{u}\|_{\mathcal{L}(X(G) ; Y(F))} \cdot \sup \left\{\left\|\left(B\left(x_{j}^{1}, \ldots, x_{j}^{n}\right)\right)_{j=1}^{\infty}\right\|_{X(G)}:\left(x_{j}^{k}\right)_{j=1}^{\infty} \in B_{X\left(E_{k}\right)}, k=1, \ldots, n\right\} \\
& =\|u\|_{X ; Y} \cdot \sup \left\{\left\|\widetilde{B}\left(\left(x_{j}^{1}\right)_{j=1}^{\infty}, \ldots,\left(x_{j}^{n}\right)_{j=1}^{\infty}\right)\right\|_{X(G)}:\left(x_{j}^{k}\right)_{j=1}^{\infty} \in B_{X\left(E_{k}\right)}, k=1, \ldots, n\right\} \\
& =\|u\|_{X ; Y} \cdot\left\|\widetilde{B}: X\left(E_{1}\right) \times \cdots \times X\left(E_{k}\right) \longrightarrow X(G)\right\|=\|u\|_{X ; Y} \cdot\|B\| .
\end{aligned}
$$

Tomando o ínfimo sobre todas as fatorações $A=u \circ B \operatorname{com} B \in \Pi_{X ; Y}$ obtemos $\|A\|_{X ; Y} \leq$ $\|A\|_{\Pi_{X ; Y} \circ \mathcal{L}}$.

Mostraremos agora que, mesmo sendo $X$ multilinearmente estável, pode-se ter $\Pi_{X ; Y} \circ$ $\mathcal{L} \neq \mathcal{L}_{X ; Y}$, o que confirma que $\mathcal{L}_{X ; Y}$ não é um ideal de composição.

Exemplo 5.3.2. Para $p \geq 1$, denotamos por $\Pi_{p}$ o ideal de Banach dos operadores lineares absolutamente $p$-somantes, ou seja, operadores que transformam sequências fracamente $p$-somáveis em sequências absolutamente $p$-somáveis (veja [34]). Na nossa notação, $\Pi_{p}=$ $\Pi_{\ell_{p}^{w}(\cdot) ; \ell_{p}(\cdot)}$. Mostremos que

$$
\mathcal{L}_{\ell_{p}^{w}(\cdot) ; \ell_{p}(\cdot)} \not \subset \Pi_{\ell_{p}^{w}(\cdot) ; \ell_{p}(\cdot)} \circ \mathcal{L}=\Pi_{p} \circ \mathcal{L}
$$


Para isso seja $E$ um espaço de Banach não reflexivo, em particular $E$ tem dimensão infinita. Tome $\varphi \in E^{*},\|\varphi\|=1$, e considere o operador bilinear contínuo

$$
A: E \times E \longrightarrow E, A(x, y)=\varphi(x) y .
$$

Por um lado, dadas sequências $\left(x_{j}\right)_{j=1}^{\infty},\left(y_{j}\right)_{j=1}^{\infty} \in \ell_{p}^{w}(E)$, existe $K>0$ tal que $\left\|y_{j}\right\| \leq K$ para todo $j$, pois toda sequência fracamente $p$-somável é limitada. Assim,

$$
\begin{aligned}
\sum_{j=1}^{\infty}\left\|A\left(x_{j}, y_{j}\right)\right\|^{p} & =\sum_{j=1}^{\infty}\left|\varphi\left(x_{j}\right)\right|^{p} \cdot\left\|y_{j}\right\|^{p} \leq K^{p} \cdot \sum_{j=1}^{\infty}\left|\varphi\left(x_{j}\right)\right|^{p} \leq K^{p} \cdot \sup _{\psi \in B_{E^{*}}} \sum_{j=1}^{\infty}\left|\psi\left(x_{j}\right)\right|^{p} \\
& =K \cdot\left\|\left(x_{j}\right)_{j=1}^{\infty}\right\|_{w, p}^{p}<+\infty,
\end{aligned}
$$

mostrando que $\left(A\left(x_{j}, y_{j}\right)\right)_{j=1}^{\infty} \in \ell_{p}(E)$. Isso prova que $A \in \mathcal{L}_{\ell_{p}^{w}(\cdot) ; \ell_{p}(\cdot)}\left({ }^{2} E ; E\right)$.

Por outro lado, de [9, Proposition p. 461] sabemos que $A$ não é fracamente compacto, ou seja, $A \notin \mathcal{L}_{\mathcal{W}}\left({ }^{2} E ; E\right)=\mathcal{W} \circ \mathcal{L}\left({ }^{2} E ; E\right)$. Pela Proposição 1.4 .8 concluímos que a linearização $A_{L}$ de $A$ não é fracamente compacta, e portanto não é absolutamente $p$-somante por [34, Theorem 2.17], isto é, $A_{L} \notin \Pi_{p}\left(E \widehat{\otimes}_{\pi} E ; E\right)$. Aplicando a Proposição 1.4 .8 uma vez mais segue que $A \notin \Pi_{p} \circ \mathcal{L}\left({ }^{2} E ; E\right)$. Isso prova (5.4).

No caso $p=1$ temos $\ell_{1}^{w}(\cdot)$ multilinearmente estável e $\mathcal{L}_{\ell_{1}^{w}(\cdot) ; \ell_{1}(\cdot)} \neq \Pi_{\ell_{1}^{w}(\cdot) ; \ell_{1}(\cdot)} \circ \mathcal{L}=\Pi_{1} \circ \mathcal{L}$, o que completa o contraexemplo.

Estabeleceremos agora a relação do ideal de polinômios $\mathcal{P}_{X ; Y}$ com o ideal de composição correspondente $\Pi_{X ; Y} \circ \mathcal{P}$.

Proposição 5.3.3. Sejam $X$ e $Y$ classes de sequências com $X$ multilinearmente estável. Então $\Pi_{X ; Y} \circ \mathcal{P} \subseteq \mathcal{P}_{X ; Y}$ e $\|P\|_{X ; Y ; 2} \leq \frac{n^{n}}{n !} \cdot\|P\|_{\Pi_{X ; Y} \circ \mathcal{P}}$ para todo $P \in \Pi_{X ; Y}\left({ }^{n} E ; F\right)$. Além disso, se $X$ for polinomialmente estável, então $\|P\|_{X ; Y ; 1} \leq\|P\|_{\Pi_{X ; Y} \circ \mathcal{P}}$.

Demonstração. Na cadeia de implicações

$$
\begin{aligned}
P \in \Pi_{X ; Y} \circ \mathcal{P}\left({ }^{n} E ; F\right) & \Longrightarrow \check{P} \in \Pi_{X ; Y} \circ \mathcal{L}\left({ }^{n} E ; F\right) \Longrightarrow \check{P} \in \mathcal{L}_{X ; Y}\left({ }^{n} E ; F\right) \\
& \Longrightarrow P \in \mathcal{P}_{X ; Y}\left({ }^{E} ; F\right)
\end{aligned}
$$

a primeira implicação segue da Proposição 1.6.14, a segunda da Proposição 5.3.1 e a terceira do Teorema 5.2.1. Da definição de ideal de composição, existem um espaço de Banach $G$, um polinômio $Q \in \mathcal{P}\left({ }^{n} E ; G\right)$ e um operador linear $u \in \Pi_{X ; Y}(G ; F)$ tais que $P=u \circ Q$. Como $\mathcal{L}_{X ; Y}$ é um hiper-ideal de Banach (Teorema 5.1.7), temos

$$
\|P\|_{X ; Y ; 2}=\|\check{P}\|_{X ; Y}=\|u \circ \check{Q}\|_{X ; Y} \leq\|u\|_{X ; Y} \cdot\|\check{Q}\| \leq \frac{n^{n}}{n !} \cdot\|u\|_{X ; Y} \cdot\|Q\| .
$$


A desigualdade desejada segue tomando o ínfimo sobre todas as fatorações $P=u \circ Q$ com $u$ em $\Pi_{X ; Y}$.

Suponha agora que $X$ seja polinomialmente estável. Para toda fatoração $P=u \circ Q$ $\operatorname{com} u$ em $\Pi_{X ; Y}$,

$$
\begin{aligned}
\|P\|_{X ; Y ; 1} & =\|\widetilde{P}\|=\sup \left\{\left\|\left(P\left(x_{j}\right)\right)_{j=1}^{\infty}\right\|_{Y(F)}:\left(x_{j}\right)_{j=1}^{\infty} \in B_{X(E)}\right\} \\
& =\sup \left\{\left\|\left(u\left(Q\left(x_{j}\right)\right)\right)_{j=1}^{\infty}\right\|_{Y(F)}:\left(x_{j}\right)_{j=1}^{\infty} \in B_{X(E)}\right\} \\
& =\sup \left\{\left\|\widetilde{u}\left(\left(Q\left(x_{j}\right)\right)_{j=1}^{\infty}\right)\right\|_{Y(F)}:\left(x_{j}\right)_{j=1}^{\infty} \in B_{X(E)}\right\} \\
& \leq\|\widetilde{u}\|_{\mathcal{L}(X(G) ; Y(F))} \cdot \sup \left\{\left\|\left(Q\left(x_{j}\right)\right)_{j=1}^{\infty}\right\|_{X(G)}:\left(x_{j}\right)_{j=1}^{\infty} \in B_{X(E)}\right\} \\
& \left.=\|u\|_{X ; Y} \cdot \sup \left\{\| \widetilde{Q}\left(\left(x_{j}\right)_{j=1}^{\infty}\right)\right) \|_{X(G)}:\left(x_{j}\right)_{j=1}^{\infty} \in B_{X(E)}\right\} \\
& =\|u\|_{X ; Y} \cdot\|\widetilde{Q}: X(E) \longrightarrow X(G)\|=\|u\|_{X ; Y}\|\cdot\| Q \| .
\end{aligned}
$$

Tomando o ínfimo sobre todas essas fatorações obtemos $\|P\|_{X ; Y ; 1} \leq\|P\|_{\Pi_{X ; Y} \circ \mathcal{P}}$.

Terminamos o capítulo mostrando que, em geral, $\mathcal{P}_{X ; Y} \neq \Pi_{X ; Y} \circ \mathcal{P}$, mesmo com $X$ multilinearmente estável.

Exemplo 5.3.4. Sejam $E, p \geq 1$ e $\varphi \in E^{*}$ como no Exemplo 5.3.2 e considere o polinômio 2-homogêneo contínuo

$$
P: E \longrightarrow E, P(x)=\varphi(x) x .
$$

O mesmo argumento do Exemplo 5.3 .2 mostra que $P \in \mathcal{P}_{\ell_{p}^{w}(\cdot) ; \ell_{p}(\cdot)}\left({ }^{2} E ; E\right)$ e $P \notin \Pi_{p}$ 。 $\mathcal{P}\left({ }^{2} E ; E\right)$. Então,

$$
\mathcal{P}_{\ell_{1}^{w}(\cdot) ; \ell_{1}(\cdot)} \not \subset \Pi_{\ell_{1}^{w}(\cdot) ; \ell_{1}(\cdot)} \circ \mathcal{P}=\Pi_{1} \circ \mathcal{P} .
$$

No caso $p=1$ temos $\ell_{1}^{w}(\cdot)$ multilinearmente estável e $\mathcal{P}_{\ell_{1}^{w}(\cdot) ; \ell_{1}(\cdot)} \neq \Pi_{\ell_{1}^{w}(\cdot) ; \ell_{1}(\cdot)} \circ \mathcal{P}=\Pi_{1} \circ \mathcal{P}$, completando o contraexemplo. 


\section{Referências Bibliográficas}

[1] Achour, D.; Alouani A.; Rueda, P.; SÁnchez-Pérez, E.A Tensor characterizations of summing polynomials, Mediterr. J. Math. 15 (2018), no. 3, Paper No. 127, $12 \mathrm{pp}$.

[2] Achour, D; Mezrag, L., On the Cohen strongly p-summing multilinear operators, J. Math. Anal. Appl. 327 (2007), 550-563.

[3] Acuña Torres, L., Adjuntos generalizados e germes de ideais de operadores lineares e polinômios homogêneos, Tese de Doutorado, IMECC, 2019.

[4] Alencar, R., An application of Singer's theorem to homogeneous polynomials, Banach spaces (Mérida, 1992), Contemp. Math., 144, Amer. Math. Soc., Providence, RI, 1993, 1-8.

[5] Alencar, R.; Matos, M. C., Some classes of multilinear mappings between Banach spaces, Publicaciones del Departamento Análisis Matemático, Universidade Complutense de Madrid, 12, 1989.

[6] Aron, R.; Schottenloher, M., Compact holomorphic mappings on Banach spaces and the approximation property, J. Funct. Anal. 21 (1976), no. 1, 7-30.

[7] Blasco, F., Complementation in spaces of symmetric tensor products and polynomials, Studia Math. 123 (1997), no. 2, 165-173.

[8] Blasco, F.; Botelho, G.; Pellegrino D.; Rueda P., Coincidence results for summing multilinear mappings, Proc. Edinb. Math. Soc. (2) 59 (2016), no. 4, $877-897$.

[9] Botelho, G., Weakly compact and absolutely summing polynomials, J. Math. Anal. Appl. 265 (2002), 458-462. 
[10] Botelho, G.; Campos, J. R., Type and cotype of multilinear operators, Rev. Mat. Complut. 29 (2016), no. 3, 659-676.

[11] Botelho, G.; Campos, J. R., On the transformation of vector-valued sequences by linear and multilinear operators, Monatsh. Math. 183 (2017), no. 3, 415-435.

[12] Botelho G.; Campos, J. R., Duality theory for generalized summing linear operators, arXiv:2010.01350v1[math.FA], 2020.

[13] Botelho, G.; Campos, J. R.; Santos, J., Operator ideals related to absolutely summing and Cohen strongly summing operators, Pacific J. Math. 287 (2017), no. 1, 1-17.

[14] Botelho, G.; Freitas, D., Summing multilinear operators by blocks: The isotropic and anisotropic cases, J. Math. Anal. Appl. 490 (2020), no. 1, 124203, 21 pp.

[15] Botelho, G.; Mujica, X., Spaces of $\sigma(p)$-nuclear linear and multilinear operators on Banach spaces and their duals, Linear Algebra Appl. 519 (2017), 219-237.

[16] Botelho, G.; Mujica, X., Ideal extensions of classes of linear operators, Studia Math. 247 (2019), no. 3, 285-297.

[17] Botelho, G.; Pellegrino, D.; Rueda, P., On composition ideals of multilinear mappings and homogeneous polynomials, Publ. Res. Inst. Math. Sci. 43 (2007), no. 4, 1139-1155.

[18] Botelho, G.; Pellegrino, D.; Teixeira, E., Fundamentos de Análise Funcional, Sociedade Brasileira de Matemática, 2012.

[19] Botelho, G.; Polac, L., A polynomial Hutton theorem with applications, J. Math. Anal. Appl. 415 (2014), no. 1, 294-301.

[20] Botelho, G.; Torkes, E. R., Hyper-ideals of multilinear operators, Linear Algebra Appl. 482 (2015), 1-20.

[21] Botelho, G.; Torres, E. R., Polynomial ideals from a nonlinear viewpoint, arXiv:1603.00246 v1[math.FA], 2016.

[22] Botelho, G.; Torres, E. R., Techniques to generate hyper-ideals of multilinear operators, Linear Multilinear Algebra 65 (2017), no. 6, 1232-1246. 
[23] Botelho, G.; Torres, E. R., Two-sided polynomial ideals on Banach spaces, J. Math. Anal. Appl. 462 (2018), no. 1, 900-914.

[24] Botelho, G.; Wood, R., On the representation of linear functionals on hyperideals of multilinear operators, Banach J. Math. Anal. 15 (2021), no. 1, Paper No. 25, $23 \mathrm{pp}$

[25] Boyd, C.; Brown, A., Duality in spaces of polynomials of degree at most n, J. Math. Anal. Appl. 429 (2015), 1271-1290.

[26] Braunss, H.; Junek, H., Ideals of Polynomials and Multilinear Mappings, unpublished notes.

[27] Bu, Q.;, SHI, Z., On Cohen almost summing multilinear operators, J. Math. Anal. Appl. 401 (2013), 174-181.

[28] Campos, J. R., An abstract result on Cohen strongly summing operators, Linear Algebra Appl. 439 (2013), 4047-4055.

[29] Campos, J. R., Cohen and multiple Cohen strongly summing multilinear operators, Linear Multilinear Algebra 62 (2014), no. 3, 322-346.

[30] Campos, J. R.; Santos, J., An anisotropic approach to mid summable sequences, Colloq. Math. 161 (2020), no. 1, 35-49.

[31] Carando, D.; Dimant, V., Duality in spaces of nuclear and integral polynomials, J. Math. Anal. Appl. 241 (2000), 107-121.

[32] Carothers, N. L., A Short Course on Banach Space Theory, London Mathematical Society Student Texts 64, Cambridge University Press, 2005.

[33] Defant, A.; Floret, K., Tensor Norms and Operator Ideals, North-Holland, 1993.

[34] Diestel, J.; Jarchow, H.; Tonge, A., Absolutely Summing Operators, Cambridge University Press, 1995.

[35] Dineen, S., Complex Analysis in Locally Convex Spaces, North-Holland, Amsterdam, 1981.

[36] Dineen, S., Complex Analysis on Infinite Dimensional Spaces, Springer Monographs in Mathematics, Springer-Verlag, London, 1999. 
[37] Dwyer, T. A. W., Convolution equations for vector-valued entire functions of nuclear bounded type, Trans. Amer. Math. Soc. 217 (1976), 105-119.

[38] Fávaro, V. V.; Jatobá, A. M., Hypercyclicity, existence and approximation results for convolution operators on spaces of entire functions, Bull. Belg. Math. Soc. Simon Stevin 26 (2019), no. 5, 699-723.

[39] FÁvaro, V. V.; Mujica, J., Hypercyclic convolution operators on spaces of entire functions, J. Operator Theory 76 (2016), 141-158.

[40] Fávaro, V. V.; Mujica, J., Convolution operators on spaces of entire functions, Math. Nachr. 291 (2018), 41-54.

[41] Fávaro, V. V.; Pellegrino, D., Duality results in Banach and quasi-Banach spaces of homogeneous polynomials and applications, Studia Math. 240 (2018), no. 2, 123-145.

[42] Floret, K., Natural norms on symmetric tensor products of normed spaces, Note Mat. 17 (1999), 153-188.

[43] Floret, K.; García, D., On ideals of polynomials and multilinear mappings between Banach spaces, Arch. Math. (Basel) 81 (2003), no. 3, 300-308.

[44] Floret, K; Hunfeld, S., Ultrastability of ideals of homogeneous polynomials and multilinear mappings on Banach spaces, Proc. Amer. Math. Soc. 130 (2002), no. 5, 1425-1435.

[45] Freitas, D., Espaços de sequências vetoriais e ideais de operadores, Dissertação de Mestrado, FAMAT, UFU, 2016.

[46] Gupta, C. P., Convolution operators and holomorphic mappings on a Banach space, Séminaire d'Analyse Moderne, No. 2, Dept. Math., Université de Sherbrooke, Québec, 1969.

[47] Gupta, C. P., On the Malgrange theorem for nuclearly entire functions of bounded type on a Banach space, Nederl. Akad. Wetensch. Proc. Ser. A73 = Indag. Math. 32 (1970), 356-358.

[48] Honig, C. S., Análise Funcional e Aplicações, Vol. I, Publicações IME-USP.

[49] KIM, S. G., Multiple weakly summing multilinear mappings and polynomials, Kyungpook Math. J. 47 (2007), no. 4, 501-517. 
[50] Lindenstrauss, J.; Tzafriri L., Classical Banach Spaces I: Sequence Spaces, Springer-Verlag, 1996.

[51] Martín, M., The version for compact operators of Lindenstrauss properties $A$ and $B$, Revista de La Real Academia de Ciencias Exactas, Fisicas y Naturales. Serie A. Matematicas 110 (2016), 269-284.

[52] Matos, M. C., On multilinear mappings of nuclear type, Rev. Mat. Univ. Complut. Madrid 6 (1993), no. 1, 61-81.

[53] Megginson, R. E., An Introduction to Banach Spaces Theory, Graduate Texts in Mathematics 183, Springer-Verlag, New York, 1998.

[54] Mezrag, L.; SAadi, K., Inclusion theorems for Cohen strongly summing multilinear operators, Bull. Belg. Math. Soc. Simon Stevin 16 (2009), no. 1, 1-11.

[55] MezraG, L.; SAAdi, K., Inclusion and coincidence properties for Cohen strongly summing multilinear operators, Collect. Math. 64 (2013), no. 3, 395-408.

[56] MujicA, J., Linearization of bounded holomorphic mappings on Banach spaces, Trans. Amer. Math. Soc. 324 (1991), 867-887.

[57] Mujica, J., Complex Analysis in Banach Spaces, Dover Publ., 2010.

[58] Muro, S.; Pinasco D.; Savransky, M., Strongly mixing convolution operators on Fréchet spaces of holomorphic functions, Integral Equations Operator Theory $\mathbf{8 0}$ (2014), no. 4, 453-468.

[59] Pietsch, A., Operator Ideals, North-Holland, 1980.

[60] Pietsch, A., Ideals of multilinear functionals, Proeedings of the Second International Conference on Operator Algebras, Ideals and Their Applications in Theoretical Physiics, Leipzig Teubner Texte Math, vol. 62 (1983), pp.185-199.

[61] Polac, L. G., O adjunto de um polinômio contínuo entre espaços de Banach, Dissertação de Mestrado, FAMAT-UFU, 2013.

[62] PopA, D., Mixing multilinear operators, Illinois J. Math. 56 (2012), 895-903.

[63] PopA, D., Multiple summability properties for Cohen p-summing operators, Math. Nachr. 288 (2015), 1028-1046. 
[64] Ribeiro, J.; Santos, F., Generalized multiple summing multilinear operators on Banach spaces. Mediterr. J. Math. 16, 5 (2019), Paper No.108, 20.

[65] Ribeiro, J.; Santos, F., Absolutely summing polynomials, Methods Funct. Anal. Topology 27 (2021), 74-87.

[66] Ryan, R. A., Applications of topological tensor products to infinite dimensional holomorphy, Thesis-Trinity College Dublin, 1980.

[67] RyAn, R. A., Introduction to Tensor Products of Banach Spaces, Springer, 2002.

[68] Santiago, A. S., Associatividade nos produtos tensoriais projetivo e injetivo, Dissertação de Mestrado, FAMAT, UFU, 2020.

[69] Serrano-Rodríguez, D. M., Absolutely $\gamma$-summing multilinear operators, Linear Algebra Appl. 439 (2013), no. 12, 4110-4118.

[70] Soares, C. A. S., Aplicações multilineares e polinômios misto somantes, Tese de Doutorado, IMECC-UNICAMP, 1998.

[71] Silva, A. R., Linearização de aplicações Multilineares Contínuas Entre Espaços de Banach e Multi-Ideias de Composição, Dissertação de Mestrado, FAMAT-UFU,2010.

[72] Torres, E. R., Hiper-ideais de aplicações multilineares e polinômios homogêneos entre espaços de Banach, Tese de Doutorado, IME-USP, 2015.

[73] Vakhania, N. N.; Tarieladze, V. I.; Chobanyan, S. A., Probability Distributions on Banach Spaces, D. Reidel Publishing Co., Dordrecht, 1987.

[74] Velanga, T., Ideals of polynomials between Banach spaces revisited, Linear Multilinear Algebra 66 (2018), no. 11, 2328-2348. 